\title{
Drinking Water Quality in Canadian First Nations Communities: \\ Do Divergent Strategies for Addressing the Issue \\ Contribute to the Problems?
}

\author{
by
}

Andrew Gajadhar

A thesis submitted to the Faculty of Graduate and Postdoctoral Affairs in partial fulfillment of the requirements for the degree of

Master of Arts

in

Public Administration

Carleton University

Ottawa, Ontario

(C) 2013, Andrew Gajadhar 
Library and Archives

Canada

Published Heritage

Branch

395 Wellington Street

Ottawa ON K1A ON4

Canada
Bibliothèque et

Archives Canada

Direction du

Patrimoine de l'édition

395 , rue Wellington

Ottawa ON K1A ON4

Canada
Your file Votre référence

ISBN: 978-0-494-94294-9

Our file Notre référence

ISBN: $978-0-494-94294-9$
NOTICE:

The author has granted a nonexclusive license allowing Library and Archives Canada to reproduce, publish, archive, preserve, conserve, communicate to the public by telecommunication or on the Internet, loan, distrbute and sell theses worldwide, for commercial or noncommercial purposes, in microform, paper, electronic and/or any other formats.

The author retains copyright ownership and moral rights in this thesis. Neither the thesis nor substantial extracts from it may be printed or otherwise reproduced without the author's permission.
AVIS:

L'auteur a accordé une licence non exclusive permettant à la Bibliothèque et Archives Canada de reproduire, publier, archiver, sauvegarder, conserver, transmettre au public par télécommunication ou par l'Internet, prêter, distribuer et vendre des thèses partout dans le monde, à des fins commerciales ou autres, sur support microforme, papier, électronique et/ou autres formats.

L'auteur conserve la propriété du droit d'auteur et des droits moraux qui protege cette thèse. $\mathrm{Ni}$ la thèse ni des extraits substantiels de celle-ci ne doivent être imprimés ou autrement reproduits sans son autorisation.
In compliance with the Canadian Privacy Act some supporting forms may have been removed from this thesis.

While these forms may be included in the document page count, their removal does not represent any loss of content from the thesis.
Conformément à la loi canadienne sur la protection de la vie privée, quelques formulaires secondaires ont été enlevés de cette thèse.

Bien que ces formulaires aient inclus dans la pagination, il n'y aura aucun contenu manquant. 


\begin{abstract}
The purpose of the thesis is to determine whether the four governance actors of First Nations drinking water (i.e., Aboriginal Affairs and Northern Development Canada, Environment Canada, Health Canada, and Band Councils) hold different theories for addressing First Nations drinking water quality issues, and whether such differences are manifested in First Nations approaches to resolving these issues. First, theories of change are developed to map out each governance actor's ideal solution for addressing First Nations drinking water quality issues. Second, the drinking water management of three First Nations communities are compared with analogous (i.e., similar size, location, and source water) non-First Nations communities. The findings suggest that approaches used by the four governance actors differ significantly, which manifests in confusion at the community level for resolving water management issues. These different approaches are measured according to financial, personnel, regulatory, and role clarity indicators.
\end{abstract}




\section{Acknowledgements}

My deepest gratitude is reserved for my supervisor, Professor Robert Shepherd. Without his oversight this thesis would not have gotten off the ground. His guidance and direction helped me to navigate the writing of this thesis and provided me with a sense of confidence in articulating my findings on this controversial topic.

Sincere thanks must be given to my four committee members, Professors Graeme Auld, Steven Montague, Gregory Poelzer, and Onita Basu. I am grateful for their participation in the thesis defence and the insightful comments they offered. I also appreciated and found great value in their challenges to the arguments of this thesis.

I am indebted to each of the anonymous interview respondents who offered invaluable insight into the management of drinking water in First Nations and non-First Nations communities. These individuals offered their time, and knowledge and in some cases critique of my work that would have been otherwise unavailable. The cooperation and openness of these individuals provided some of the most significant information for informing this work.

Finally, my thanks must be extended to my family and friends for their continued support and encouragement throughout the entire thesis process. First, my thanks go out to my friends from Carleton University who supported my undertaking of a thesis project and were there to hear my complaints throughout the process. My friends from Saskatoon have similarly offered me the time, space, and patience for undertaking this task. The support of Kelly Donald was instrumental for enabling me to continue through to the end of the process, as having someone to talk out ideas, challenges, and frustrations kept my focus on task and my outlook positive. My brothers, Mark and Grant Gajadhar and their families, Amanda, Casey, Terran, Hailee, and Sara, have each contributed in their own ways by also offering support in times of need. Lastly, without my parents, Alvin and Doris Gajadhar, I would not have had the means or the courage to undertake this project. I am grateful and fortunate to have been surrounded by such wonderful people. 


\section{Table of Contents}

Chapter 1: Drinking Water Management Frameworks and Drinking Water Quality of First Nations and Non-First Nations Communities ................................................................ 1

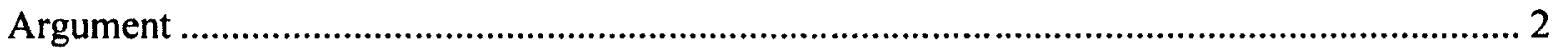

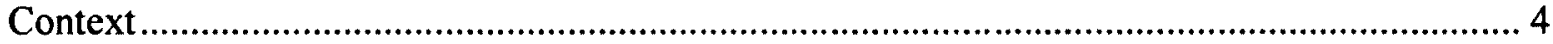

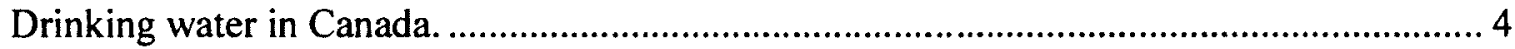

Drinking Water Quality in First Nations Communities ................................................ 8

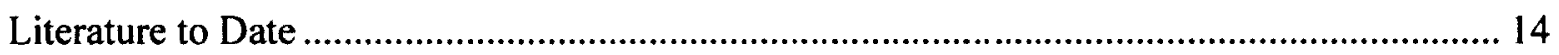

Drinking water management in First Nations communities. .......................................... 14

Evaluations of drinking water quality of non-First Nations communities........................... 18

Evaluations of drinking water quality of First Nations communities. ................................ 21

Government conducted evaluations of drinking water qual ity..................................... 22

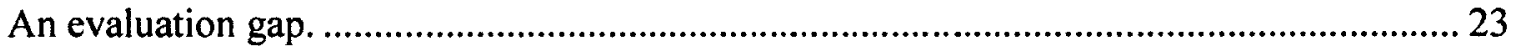

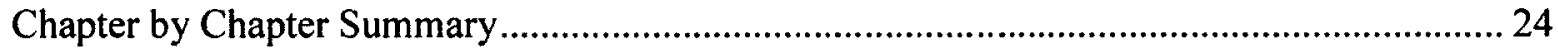

Chapter 2: Methodology for Addressing the Research Gaps..................................................... 28

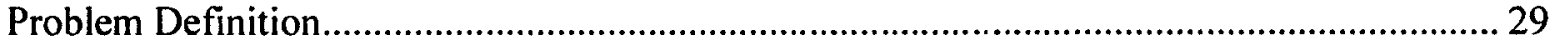

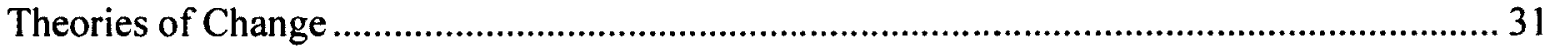

Comparing First Nations Communities with Analogous Non-First Nations

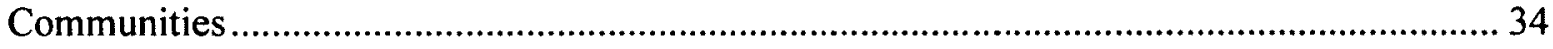

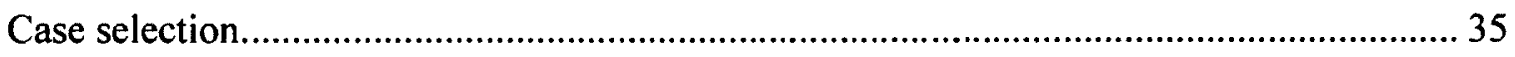

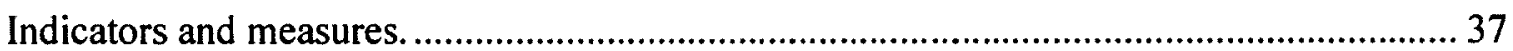

Chapter 3: First Nations Governance Actors' Diverse Theories for Remedying Drinking Water Quality Issues ....................................................................................................... 40

Aboriginal Affairs and Northern Development Canada .................................................... 40

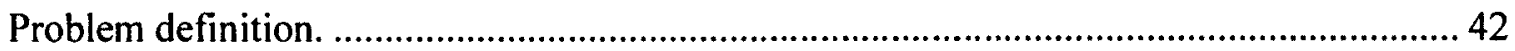

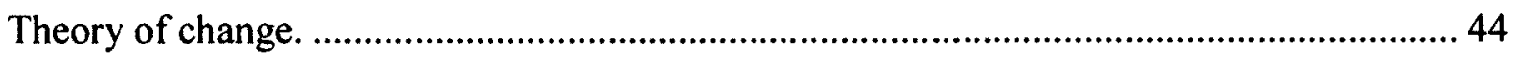

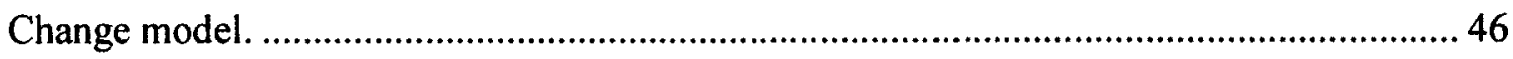

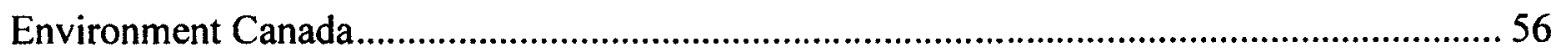

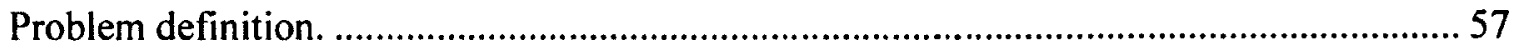

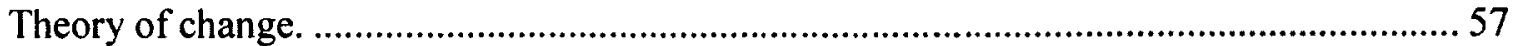

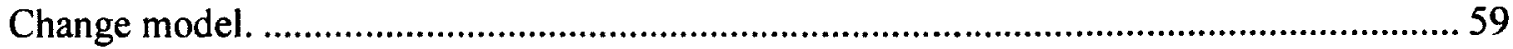

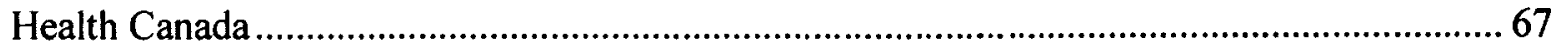




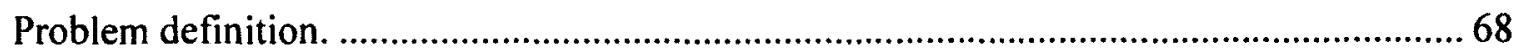

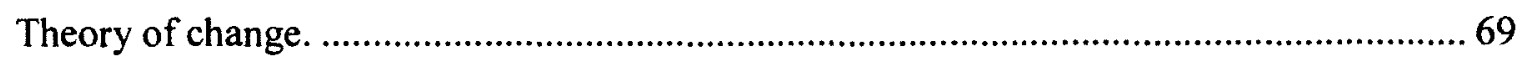

Change model.

Assembly of First Nations and Chiefs of Ontario .............................................................. 78

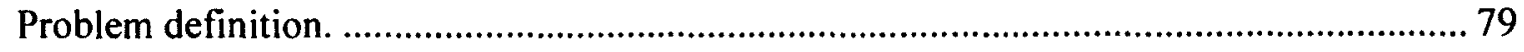

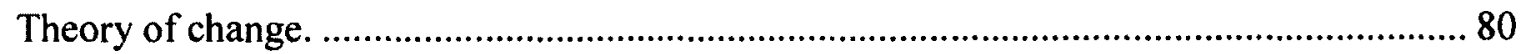

Change model. ....................................................................................................... 81

Comparison of Governance Actors' Approaches ………….............................................. 90

Comparison of governance actors' problem definitions.................................................... 90

Comparison of governance actors' theories of change........................................................ 92

Comparison of governance actors' change models. ......................................................... 94

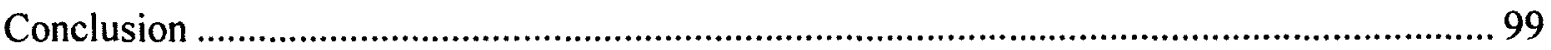

Chapter 4: A Comparison of Communities using the Same Drinking Water System ............... 102

Community Descriptions ……………………………….................................................. 104

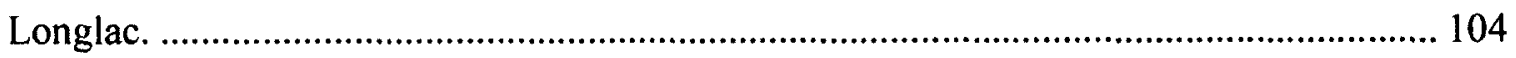

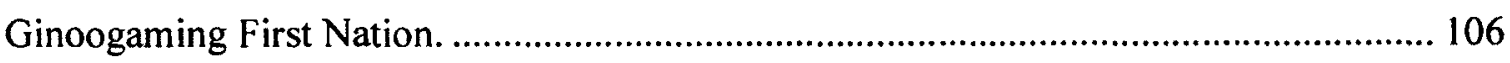

Comparison of community descriptions. ................................................................... 107

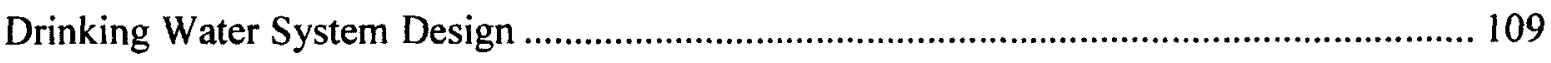

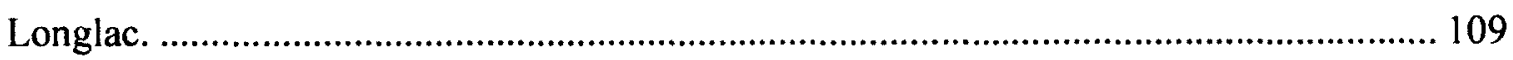

Ginoogaming First Nation. ................................................................................. 110

Comparison of community drinking water system design. ............................................... 110

Source Water Protection ........................................................................................... 111

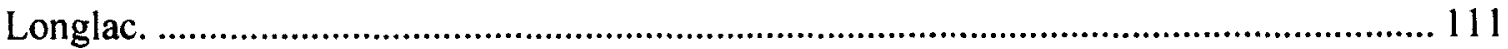

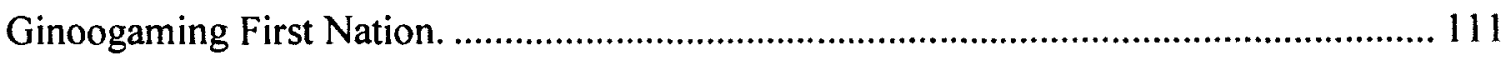

Comparison of community source water protection......................................................... 111

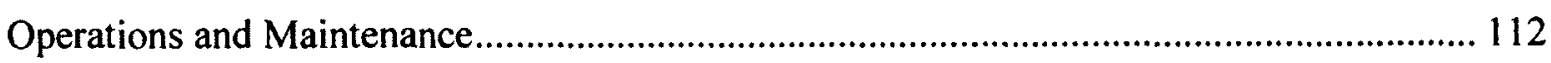

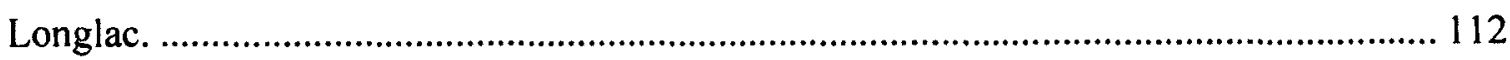

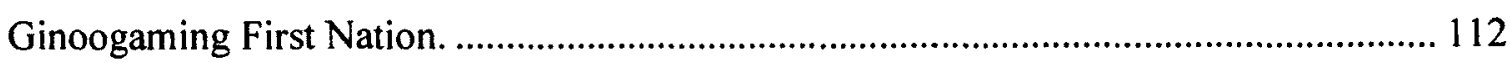

Comparison of community operations and maintenance................................................. 113

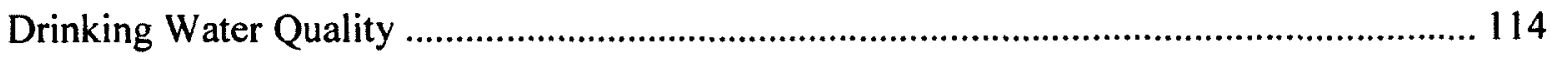

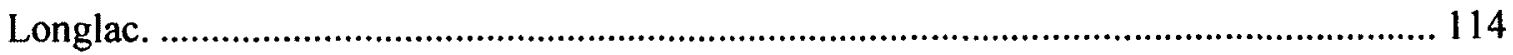

Ginoogaming First Nation. ................................................................................. 114

Comparison of community drinking water quality ...................................................... 115

Improvement Recommendations ................................................................................... 116 


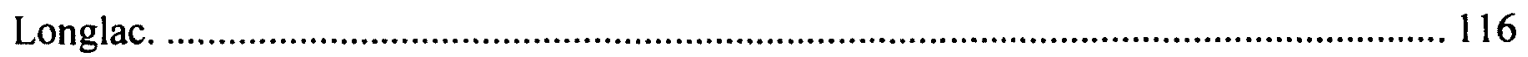

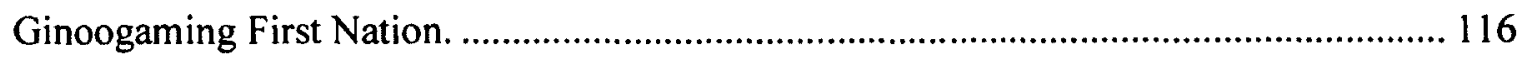

Comparison of community improvement recommendations............................................. 118

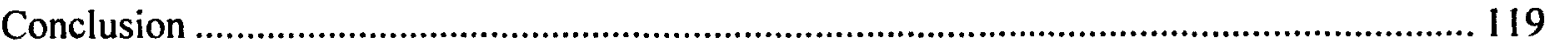

Chapter 5: A Comparison of Communities using Groundwater as their Source of

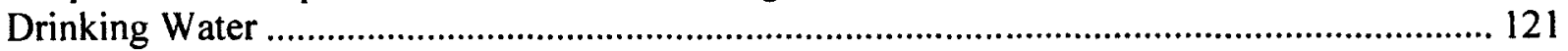

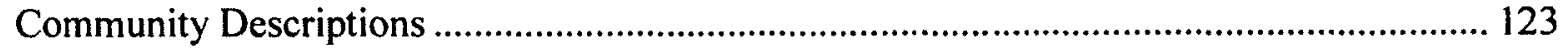

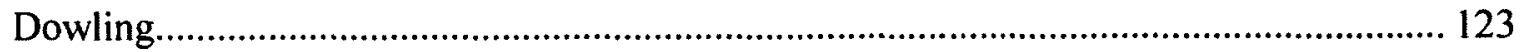

Sagamok Anishnawbek First Nation. ............................................................................ 124

Comparison of community descriptions. ..................................................................... 126

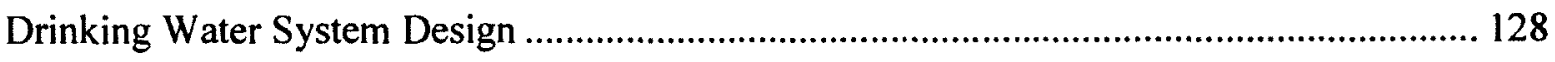

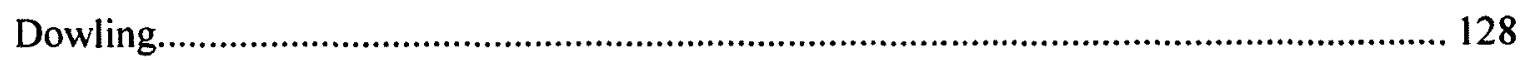

Sagamok Anishnawbek First Nation. .......................................................................... 129

Comparison of community drinking water system design. ............................................... 129

Source Water Protection .......................................................................................... 130

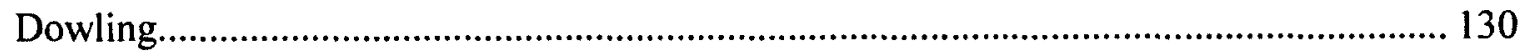

Sagamok Anishnawbek First Nation. ...................................................................... 130

Comparison of community source water protection..................................................... 131

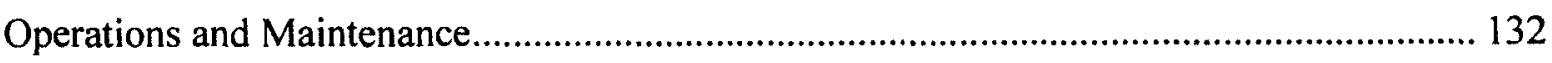

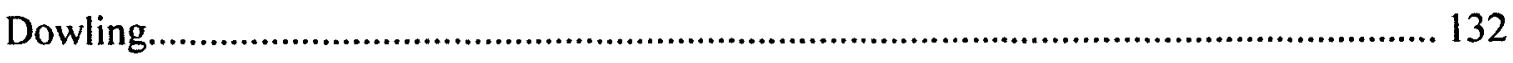

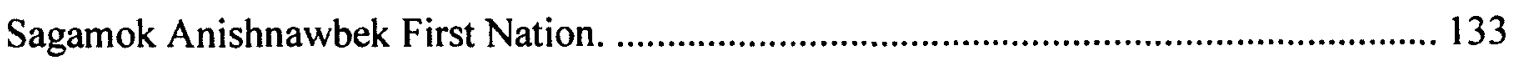

Comparison of community operations and maintenance.................................................. 134

Drinking Water Quality ……………….................................................................. 135

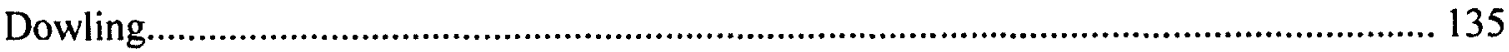

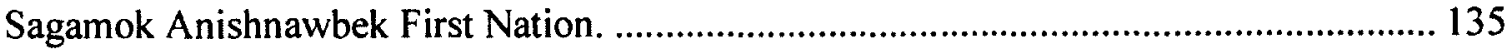

Comparison of community drinking water quality......................................................... 136

Improvement Recommendations ............................................................................... 137

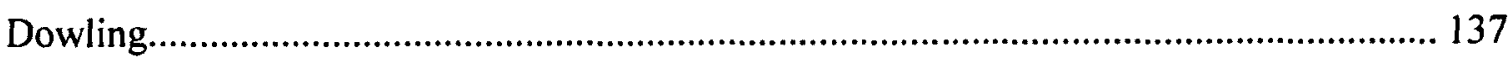

Sagamok Anishnawbek First Nation. ...................................................................... 137

Comparison of community improvement recommendations............................................ 138

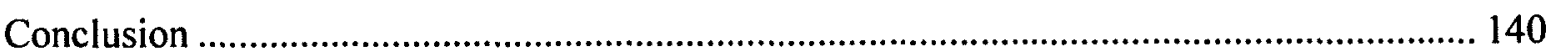

Chapter 6: A Comparison of Communities using Surface Water as their Source of Drinking Water

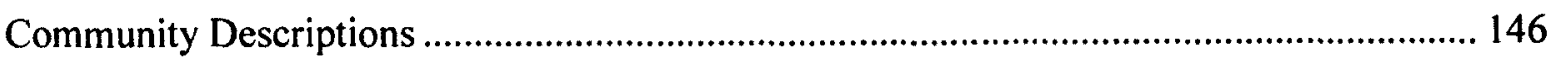

Red Lake. 
Pikangikum First Nation..................................................................................... 147

Comparison of community descriptions. ...................................................................... 148

Drinking Water System Design ............................................................................... 151

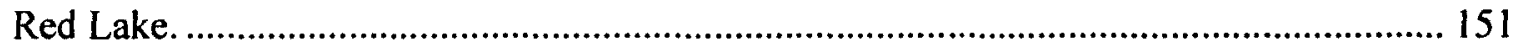

Pikangikum First Nation. ....................................................................................... 151

Comparison of community drinking water system design. ............................................... 153

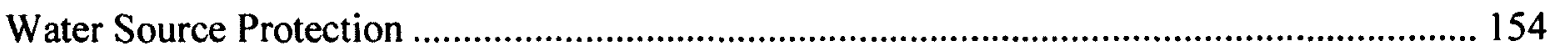

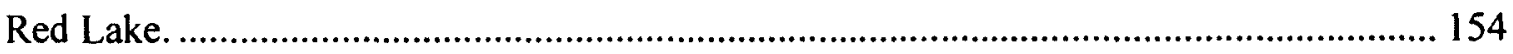

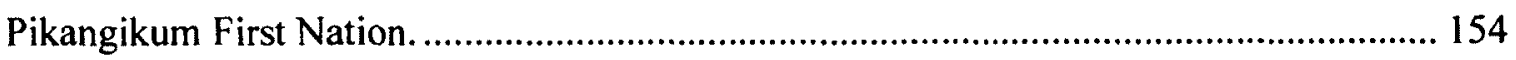

Comparison of community source water protection....................................................... 154

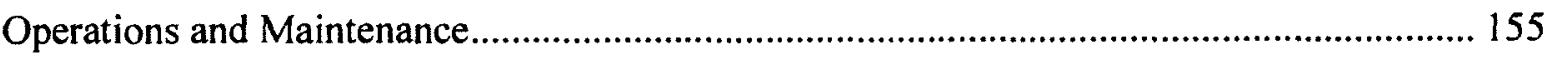

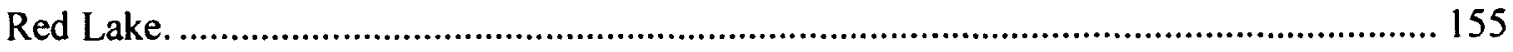

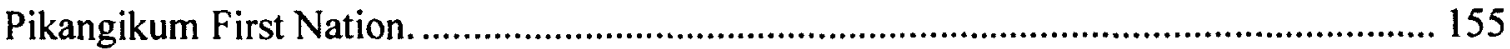

Comparison of community operations and maintenance................................................. 156

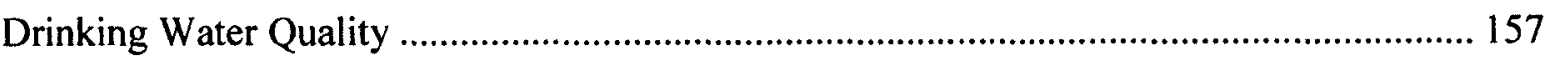

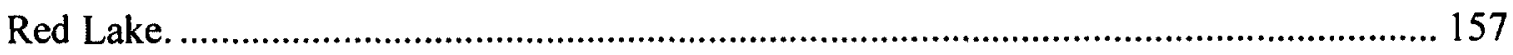

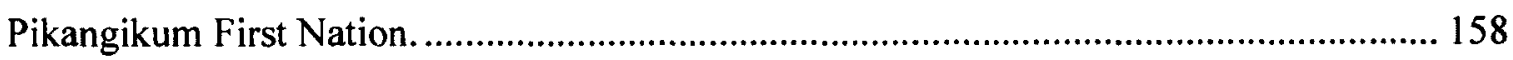

Comparison of community drinking water quality......................................................... 159

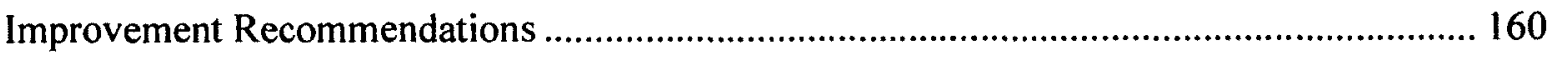

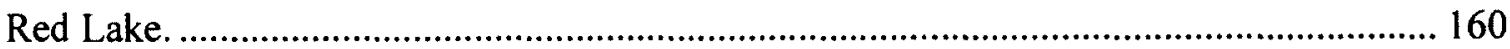

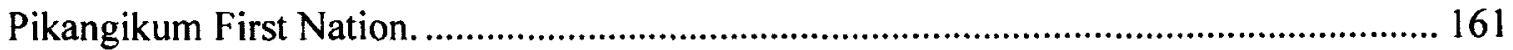

Comparison of community improvement recommendations.............................................. 162

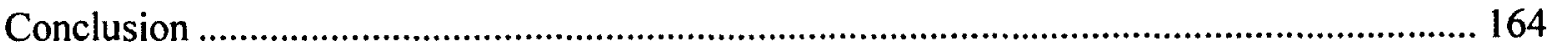

Chapter 7: Concluding Observations Regarding Drinking Water Quality Differences between Analogous First Nations and Non-First Nations Communities.................................... 167

Sub-Question 1: Defining the Problem.............................................................................. 168

Sub-Question 2: Solutions for Remedying the Problem...................................................... 169

Sub-Question 3: Divergent Solutions Contributing to the Problem ........................................ 169

Differences in financial capacity. .............................................................................. 170

Differences in human resource capacity................................................................... 172

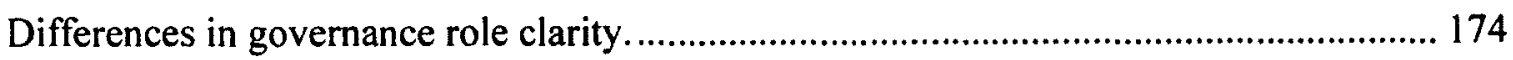

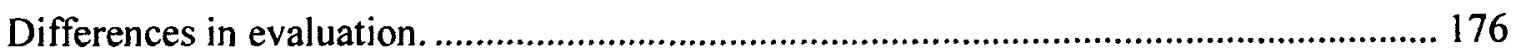

Differences in regulation........................................................................................ 178

Differences in improvement recommendations............................................................. 179

Water source disparity. ........................................................................................... 182 
Recommendations

Recommendation 1: Improving trust.

Recommendation 2: Improving role definition

Recommendation 3: Improve coordination.

Considerations Moving Forward

Consideration 1: Restructure funding relationships.

Consideration 2: Amalgamating drinking water services.

Consideration 3: Restructuring funder and compliance monitoring roles.

Limitations

Implications and Conclusions

References.

Glossary of Terms

Annex 1: Departments/Ministries Responsible for Drinking Water Quality by

Province/Territory

Annex 2: Frequency of Factors Pertaining to Specific First Nations Drinking Water Quality Referenced by Governance Actor

Annex 3: Percentage of Total Factor References Pertaining to First Nations Drinking Water Quality as Referenced by each Governance Actor .

Annex 4: Reference list for Documents Reviewed from Aboriginal Affairs and Northern Development Canada.

Annex 5: Reference list for Documents Reviewed from Environment Canada

Annex 6: Reference list for Documents Reviewed from Health Canada

Annex 7: Reference list for Documents Reviewed from the Assembly of First Nations and Chiefs of Ontario.

Annex 9: Interview Questions Used for the Purposes of this Study

Annex 10: Huey T. Chen's Action Theory Model

Annex 11: Water Quality Parameters for Municipal Type Agreement Source ........................ 235

Annex 12: Drinking Water System Improvements for Longlac ........................................... 238

Annex 13: Drinking Water System Improvements for Ginoogaming .................................... 239

Annex 14: Greater Sudbury Organizational Chart: Water/Wastewater Services ...................... 240

Annex 15: Water Quality Parameters for Groundwater Source ............................................ 241

Annex 16: Drinking Water System Improvements for Dowling ............................................ 244

Annex 17: Drinking Water System Improvements for Sagamok ........................................... 245

Annex 18: Water Quality Parameters for Surface Water Source ............................................. 246

Annex 19: Drinking Water System Improvements for Red Lake............................................. 248

Annex 20: Drinking Water System Improvements for Pikangikum ....................................... 249 


\section{List of Tables}

Table 1: Problem Definitions Indicators and Measures.......................................................... 31

Table 2: Theories of Change Indicators and Measures....................................................... 32

Table 3: Matched-Pair Case Indicators and Measures.......................................................... 39

Table 4. AANDC Action Theory: Planning............................................................................ 47

Table 5. AANDC Action Theory: Investment ............................................................................. 49

Table 6. AANDC Action Theory: Training …........................................................................... 50

Table 7. AANDC Action Theory: Operations and Maintenance .............................................. 51

Table 8. AANDC Action Theory: Legislation ........................................................................... 52

Table 9: AANDC Action Theory: Regulation....................................................................... 52

Table 10. AANDC Action Theory: Enforcement .................................................................... 53

Table 11. AANDC Action Theory: Continuous Monitoring...................................................... 54

Table 12. AANDC Action Theory: Source Water Protection .................................................... 55

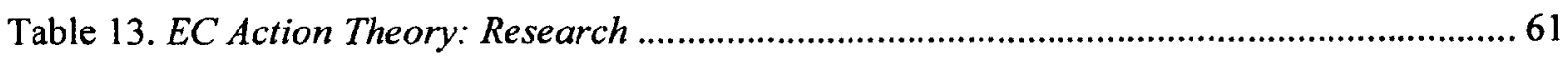

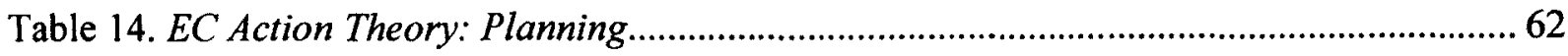

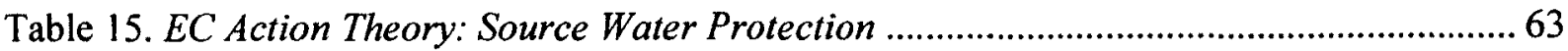

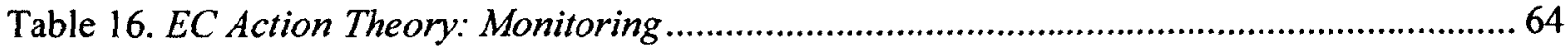

Table 17. EC Action Theory: Infrastructure Investment .........................................................6 65

Table 18. EC Action Theory: Operations and Maintenance ..................................................... 66

Table 19. EC Action Theory: Legislation and Regulation .......................................................67 67

Table 20. HC Action Theory: Research ................................................................................. 72

Table 21. HC Action Theory: Continued Monitoring ............................................................. 73

Table 22. HC Action Theory: Capacity Building.................................................................. 74

Table 23. HC Action Theory: Develop Procedures ..................................................................... 75

Table 24. HC Action Theory: Implement Treatment ….......................................................... 76

Table 25. HC Action Theory: Crisis Mitigation ..................................................................... 77

Table 26. HC Action Theory: Regulation and Enforcement ................................................... 78

Table 27. AFN and CoO Action Theory: Engagement …........................................................... 83

Table 28. AFN and CoO Action Theory: Needs Assessments .................................................... 84

Table 29. AFN and CoO Action Theory: Comprehensive Planning ........................................... 85

Table 30. AFN and CoO Action Theory: Infrastructure Development ...................................... 85

Table 31. AFN and CoO Action Theory: Training …............................................................... 86 
Table 32. AFN and CoO Action Theory: Operations and Maintenance .................................... 87

Table 33. AFN and CoO Action Theory: Source Water Protection ........................................... 88

Table 34. AFN and CoO Action Theory: Legislation ............................................................. 89

\section{List of Illustrations}

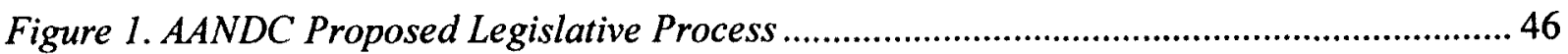

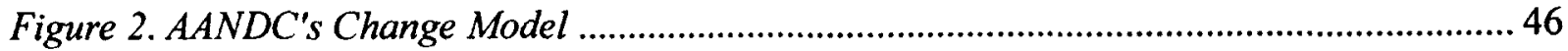

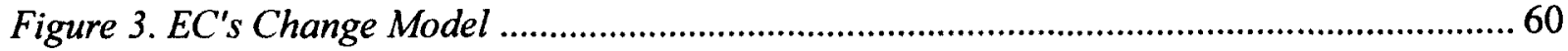

Figure 4. AFN's Proposed Policy Development Process ......................................................... 89 


\section{List of Acronyms}

AANDC: Aboriginal Affairs and Northern Development Canada (Federal)

AFN: Assembly of First Nations

CBWM: Community-Based Water Monitor

CCME: Canadian Council of Ministers of the Environment

CoO: Chiefs of Ontario

DWA: Drinking Water Advisory

DWO: Drinking Water Order

EC: Environment Canada (Federal)

EHO: Environmental Health Officer

FTE: Full Time Equivalent

GCDWQ: Guidelines for Canadian Drinking Water Quality

GEMS: Global Environmental Monitoring System

HC: Health Canada (Federal)

MOE: Ministry of the Environment (Ontario)

MOI: Ministry of Infrastructure (Ontario)

MTA: Municipal Type Agreement or Municipal Transfer Agreement (used interchangeably)

SCADA: Supervisory Control and Data Acquisition

SCAMP: Strategic Capital Asset Management Plan

The Protocol: Protocol for Centralised Drinking Water Systems in First Nations Communities

WDE: Water Disease Event

WSP: Water Safety Plan

WTO: Water Treatment Operator or Water Treatment Plant Operators (used interchangeably) 


\section{Chapter 1: Drinking Water Management Frameworks and Drinking Water Quality of First Nations and Non-First Nations Communities}

The following thesis is part of the requirements of a Master's of Arts thesis in Public Administration and it focuses on the public management of drinking water on First Nations reserves in Canada. Using a program evaluative lens, the thesis takes particular aim at the drinking water management framework of these communities. The research question this thesis seeks to address is: Does the governmental management framework surrounding drinking water systems on First Nations reserves contribute to the challenges for improving drinking water quality in these communities? Selected subquestions in addressing this question are:

1. How do four federal and Aboriginal governance actors involved (i.e., Aboriginal Affairs and Northern Development Canada, Health Canada, Environment Canada, and the First Nations governments - i.e., Chief and Council) in managing drinking water on First Nations reserves define the problem regarding First Nations' drinking water quality?

2. How do these actors' solutions for remedying the problem differ; and, in what specific ways do they diverge?

3. Are the differences in solutions for remedying drinking water quality issues on First Nations reserves contributing to the drinking water quality issues? Together the analysis of these three sub-questions triangulate in addressing the overarching research question. The first sub-question is designed to provide specific context regarding how each actor has framed the issue at hand, which affects the way in which the problem is addressed. The second sub-question provides context with regard to 
how the four actors approach the problem and illustrates the divergence of these approaches for addressing the problem. The third sub-question builds from the second, as comparisons of risk for water contamination are made across different water system management frameworks.

\section{Argument}

The governance structure for managing First Nations reserves' (hereafter referred to as First Nations communities ${ }^{1}$ ) drinking water is complicated, as there are four primary actors, including Aboriginal Affairs and Northern Development Canada (AANDC), Health Canada (HC), Environment Canada (EC), and the First Nation government (Swain, Louttit, \& Hrudey, 2006). As a result of this management framework, it is hypothesized that there are four distinct problem definitions regarding national drinking water quality on reserves. It follows that there are four independent solutions for addressing the problem, which are a series of interventions and/or activities designed to address a particular issue commonly referred to as theories of change. The differentiation between the four actors' change theories is a key component contributing to the problem and in preventing the development of a comprehensive solution because the four actors are equal in terms of government level and status. Therefore, despite the complementary components of their roles, each actor acts as an independent agent in developing theoretical solutions. The thesis illustrates the divergence that has occurred in developing these solutions, which may be seen as an impediment for developing a legitimate and collaborative approach for addressing the issues. Moreover, identification of such

\footnotetext{
${ }^{1}$ This term was changed as per advice by the anonymous First Nations governance actor interviewee (personal communication, April 4, 2012). This individual stated that many First Nations people find the term 'reserve' derogatory and prefer the term community, as it is more congruent with their beliefs.
} 
differences may help to advocate for inclusion of all four actors in coming to a common solution (e.g., AFN, 2011; Graham, 2003; Mackinnon, 2005; Wilson, 2004).

There is a common perception that drinking water in First Nations communities is of poorer quality than that of other Canadians (e.g., O'Connor, 2006b; Liu, 2011; Swain, Louttit, \& Hrudey, 2006); however, no comparisons have been made to illustrate that this is the case. There is a variety of evaluation techniques used for addressing drinking water quality on First Nations reserves as well as in non-First Nations communities. Such evaluations have not included both First Nations and non-First Nations communities. Additionally, evaluations on drinking water quality in analogous First Nations and nonFirst Nations communities have not been compared. This gap is relevant for the current thesis because each of the two community types have different drinking water management frameworks. For example, First Nations communities' drinking water quality management is guided by the federally implemented Protocol for Centralised Drinking Water Systems in First Nations Communities, whereas non-First Nations are subject to standards or guidelines established by their respective provinces. Therefore, accounting for other factors known to contribute to poor drinking water quality such as location (e.g., Hrudy, 2008; Petersen, n.d.), population size (Graham, 2002; Rollins, Frehs, Tate, \& Zachariah, 1997), and water source (Cool et al., 2010; 2011) enables a comparison of the impact of the different drinking water management frameworks of the two community types. Differences between the evaluations of both community types' drinking water are explored and lend to the argument that divergence between the management actors of First Nations communities' drinking water has been a contributing factor to the drinking water quality challenges of these communities. 


\section{Context}

Drinking water in Canada. Drinking water in Canada has been an issue for many communities throughout Canada. Between 1974 and 2001 there were 99 infectious disease outbreaks primarily due to oversight failures in publically governed drinking water systems (Schuster, et al., 2005). These outbreaks constituted two or more individuals contracting infectious diseases from a public water source. The treatment processes, human error, or a lack of stringent techniques and enforcement were HC's most commonly cited reasons for the occurrence of these incidents. In their analysis of the reported infectious disease outbreaks, Schuster et al. indicated that there was a high level of discrepancy in the format and specifications in each outbreak report. It was suggested that the reason for such differentiation is that these outbreaks occurred in several different regions across Canada.

A 1996 study of more than 72 Canadian municipalities' drinking water sources illustrated that $18.2 \%$ of treated water samples tested positive for coliforms above the recommended margin of safety (Wallis, Erlandsen, Isaac-Renton, Olson, Robertson, \& Van Keulen). These drinking water quality issues have persisted over time. Indeed, a Canadian Medical Association snapshot of April 7, 2008 revealed that 1,766 boil-water advisories were in effect throughout Canada (Eggerston, 2008). The researcher further notes that Ontario alone issued 679 boil water advisories between 2006 and 2008. It is conceivable that several instances of poor water quality may exist without such advisories in place. Further, this CMA study does not report on other Drinking Water Advisories (DWAs) that were in effect. Other types of DWAs included do not consume and do not 
use advisories (AANDC, $201 \mathrm{lb}$ ). Therefore, drinking water quality issues in Canada have been significantly underreported nationally.

Perhaps the most well-known drinking water quality issue in Canada occurred in Walkerton, Ontario. In 2000 the town experienced E. coli contamination due to rain flooding, which caused farm animal sewage to flow into the community's drinking water supply (Snider, 2004). This crisis rendered more than 1,300 people ill and seven individuals dead. In a discourse analysis of government reports, it was revealed that the blame for the incidence was placed on two water treatment plant operators who failed to properly follow provincial and federal guidelines (Mullen, Vladi, \& Mills, 2006). However, the public inquiry conducted by Justice O'Connor (2002a) indicated that the root causes of the crisis went beyond the alleged incompetence of two individuals. Specifically, these water treatment plant operators were hired without first receiving adequate training from Ontario provincial government and were able to maintain high positions of authority despite a myriad of concerns from other local authorities. Such concerns were brought to the attention of both the provincial and federal departments prior to the E. coli outbreak. Further, it was noted that the MOE failed to protect Walkerton citizens from this water contamination by relaxing its enforcement of water treatment regulations ( $\mathrm{O}^{\prime}$ Connor). Many of Justice O'Connor's recommendations related to providing adequate financial support for Walkerton's water treatment plant operation, maintenance, and staffing.

Since the Walkerton crisis, a host of changes to drinking water management in Canada have been made. The most stringent of these changes occurred in Ontario. The province is now considered to have one of the most stringent drinking water quality 
policies in Canada ( $\mathrm{O}^{\prime}$ Connor, 2002b). Following the event, the province first established drinking water quality regulations through the Safe Drinking Water Act, 2002, meaning that they are legally enforceable by the province (Holme, 2003). The Ministry of Infrastructure's (MOI) and MOE's budgets were also increased to address problems with drinking water system infrastructure and water treatment plant operator training. Therefore, the management regime of Ontario's drinking water has been consolidated (Holme). Further, drinking water in Ontario is under the management of only two primary actors, the provincial government, driven by the MOE, and the community overseeing its own water source. Other provincial departments have a limited role in water infrastructure (MOI) and municipal governance structure [Ministry of Municipal Affairs and Housing (MAH)]; however, the MOE is responsible for overall oversight including the enforcement of the legislated regulations. This management framework is tiered, as the direct oversight of drinking water systems occurs at the municipal level (occasionally with financial assistance from provincial ministries) and enforcement of regulatory standards occurs at the provincial level. Therefore, these two actors work together in a hierarchical arrangement.

Drinking water in Canada: A complex management framework. Drinking water policies in Canada are affected by several provisions related to water pollution, wastewater management, industry, agriculture, and fisheries among a host of other domains (Willms \& Shier, 2006). That is, legislation and regulations for these domains must be considered when drafting or amending drinking water quality legislation. Such legislation pertinent to First Nations drinking water quality policy includes:

- Canada Water Act, 1985;

- Canadian Environmental Protection Act, 1999; 
- Department of Health Act, 1996;

- Department of Indian Affairs and Northern Development Act, 1985;

- Indian Act, 1985;

- First Nations Land Management Act, 1999; and

- First Nations Commercial and Industrial Development Act, 2005 (Swain, Louttit, \& Hrudey, 2006).

As a result of this broad mix of legislation, the management of drinking water in Canada has become a complex issue, as the actors involved span several public domains.

At the federal level EC is responsible for overseeing water pollution policies; $\mathrm{HC}$ is in charged with oversight on mandates pertaining to safe drinking water monitoring at tap and guidelines and procedures for conducting such work (HC, 2011a); and the AANDC (formerly INAC) is responsible for overseeing First Nations community water provisions (Willms \& Shier, 2006). Each province also presides over its own drinking water policies (Hill, et al., 2008). Water quality is within provincial jurisdiction, as natural resources fall under section 92 of the Constitution Act, 1867. Policies or guidelines set by the federal government are considered as recommendations or guidelines (Davies \& Mazumder, 2003). However, since the Walkerton incident, most provinces' water quality regulations or guidelines are more stringent than federal guidelines (Holme, 2003). Provincial ministries of environment or health oversee the regulations and have power to fine for non-compliance (Isfeld, 2009; see Annex 1). The regulations and guidelines can become complex because provinces delegate the authority to preside over local water quality to local (i.e., municipal, township, etc.) governments, which have autonomy over the funding and actual management of their own systems (Hill, Furlong, Bakker, \& Cohen, 2008).

As a result of this drinking water management framework, there is little consistency for managing drinking water quality across Canada. Specifically, region-by- 
region (often provincial) regulations, reporting, and compliance with regulations/guidelines for ensuring safe drinking water have been divergent historically (Isfeld, 2009). First Nations peoples have faced a particularly complex drinking water management regime given that they are subject to varying provincial standards.

Drinking Water Quality in First Nations Communities

Dr. Hans Peterson, founder of the Safe Drinking Water Foundation, has indicated that residents of non-First Nations rural communities are the 'next' First Nations peoples, implying that drinking water quality of First Nations communities is currently poorer than in other Canadian communities (SDWF, 2011). This assertion is consistent with many other experts' judgements on drinking water in Canada. For example, Justice O'Connor (2002b) indicated that First Nations' drinking water is of some of the worst quality in Canada. Harry Swain, chair of the Expert Panel on Safe Drinking Water for First Nations, indicated a higher risk for drinking water contamination on reserves (Swain, Louttit, \& Hrudey, 2006).

A 1995 study by INAC indicated that roughly 201 (25\%) of First Nations communities' water systems were at high-risk for drinking water contamination (St. Germain, 2007). These drinking water systems had "several major deficiencies related to issues such as water source, design, operation, reporting and operator training or certification and there is a high probability that any problem could result in unsafe water" (St. Germain). In 2001, a follow-up assessment revealed that 234 (29\%) of First Nations communities' drinking water systems posed significant risk for water contamination (INAC, 2003). However, in 2007 INAC classified only 97 (17\%) First Nations communities high-risk for water contamination. After concentrated efforts (and spending 
nearly $\$ 1.25$ billion) that number was reduced to $48(9 \%)$ (INAC, 2010d). Interestingly, the government of Canada had been criticized for these estimations, as other studies have illustrated a direr picture of drinking water quality of First Nations communities. For example, Murdocca (2010) suggested that as many as $224(40 \%)$ communities are actually at risk for water contamination. Similarly, Smith et al. (2006) found that 50 (89\%) Alberta-based drinking water systems in First Nations communities were high-risk for water contamination. They noted that the 2001 INAC assessment only identified 14 $(28 \%)$ of these 50 as high-risk despite the fact that the two studies definitions for highrisk were quite similar. They suggested that there were several problems with the management structure of water in First Nations communities. In 2011, AANDC released the results of an independent national risk assessment, which articulated that there are currently 314 (39\%) First Nations community drinking water systems at high-risk for contamination.

Perhaps the most widely known example of drinking water quality issues in a First Nations community is Kashechewan in northern Ontario. In 2005, more than half of Kashechewan's population of 1,700 was relocated after learning that their drinking water supply had been contaminated with E. coli due to human sewage overflow (Murdocca, 2010). The community had been on and off boil-water advisories for the seven years leading up to the incident. The causes of this crisis were primarily linked to untrained treatment plant operators, a lack of federal enforcement of guidelines, failure of federal departments to act in response to the seven years of advisories, and limited capital to support the operation and maintenance of water management systems (Murdocca). 
Therefore, each of the governance actors failed in some way to support (or oversee) drinking water quality in this community.

Since the Kashechewan crisis, a variety of work has been conducted on the federal level. Several commissions have been formed including the Expert Panel on Safe Drinking Water for First Nations (Swain, Louttit, \& Hrudey, 2006; Willms \& Shier, 2006), and a Senate committee report on safe drinking water for First Nations (St. Germain, 2007). Moreover, the federal government announced that First Nations' drinking water quality is a top priority in several press releases. The 2010 Speech from the Thrown articulated that "First Nations' safe drinking water and effective waste-water treatment" is a national priority (GOC, 2010). As such, $\$ 330$ million of the 2010 federal budget was allocated to First Nations water quality over two years (Auclair \& Simone, 2010). This budget allocation has since extended for another two years (GOC, 2012a).

AANDC invested $\$ 1.25$ billion in water and wastewater infrastructure in First Nations communities between the fiscal years 2006-2010 (INAC, 2006). Such funding has come from three primary sources including INAC's Capital Facilities and Maintenance Program ( $\$ 820$ million), the First Nations Water and Wastewater Action Plan (\$431 million), and Canada's Economic Action Plan (\$68.6 million). The majority of these funds have been spent on improving the water and wastewater systems. AANDC has also estimated that the amount spent on First Nations community water quality will total $\$ 2.5$ billion as of fiscal year 2014 (AANDC, 2011a). It is anticipated that by 2016 , the department will have invested in almost $25 \%$ of the 314 First Nations' water systems the National Assessment identified as high overall risk. Despite these efforts, the Government of Canada has been highly criticized for not improving federal legislation 
and the management framework of drinking water in First Nations communities. Instead it has focused only on funding issues (Eggerston, 2008).

Drinking water quality management in First Nations communities. Although the Constitution Act, 1867 establishes that drinking water on reserves is under the auspices of the federal government, individual departments have been delegated specific responsibilities (Auclair \& Simone, 2010). First, AANDC is charged with presiding over funding used for the capital costs of water plants and piped systems, and allocating funds to each reserve such that $80 \%$ the operating and maintenance costs are covered by the federal government (Swain, Louttit, \& Hrudey, 2006). Similarly, the department provides all funding for the training and certification of water treatment operators (WTOs) (INAC, 2010a). AANDC is also responsible for monitoring compliance with drinking water standards through funding agreements, some of which are made in partnership with the provinces (e.g., Ontario). However, such agreements are contentious and rarely enforced. It should also be noted that Public Works assists in procuring private sector services relating to First Nations' drinking water, such as construction or third party water sample testing (Swain, Louttit, \& Hrudey). AANDC also provides advice to First Nations with regard to the design, construction, and operation and maintenance of water and wastewater systems (INAC, 2010a).

Second, $\mathrm{HC}$ is responsible for training Community-Based Water Monitors (CBWM) as well as overseeing drinking water monitoring at tap of all reserve communities below the $60^{\text {th }}$ parallel (HC, 2011a). The department's Environmental Health Officers (EHOs), with permission of First Nations, monitors adherence with the Procedure Manual for Safe Drinking Water in First Nations Communities South of $60^{\circ}$. It 
creates and maintains procedures and guidelines for drinking water systems in First Nations communities, and works with First Nations communities south of $60^{\circ}$ in identifying potential problems and provides advice on issuing drinking water advisories (HC, $2011 \mathrm{a})$. In addition, $\mathrm{HC}$ reviews the design of drinking water systems from a public health perspective.

Third, EC provides advice and guidance to First Nations in developing source water protection and sustainable water usage planning (INAC, 2010a).

Despite the roles of these federal government actors, individual First Nations governments oversee the operation and maintenance of their local water systems, including the design, construction, and operation and maintenance, and monitoring of the water systems. Individual actors within the communities also bear responsibilities pertaining to drinking water quality (Swain, Louttit, \& Hrudey, 2006). Band councils (chiefs, councillors, utility directors, infrastructure managers, and public works supervisors) are responsible for ensuring that drinking water systems are designed, constructed, and upgraded in accordance with AANDC's protocols for drinking water systems (INAC, 2010b). WTOs are responsible for the day-to-day operation and maintenance of water systems. Finally, CBWMs assist $\mathrm{HC}$ in monitoring water samples throughout the distribution system. Despite EC involvement, band councils retain the authority to set water pollution provisions within the boundaries of their First Nations' land. Band councils are responsible for ensuring that WTOs are trained in accordance with provincial guidelines. Additionally, they set compensation for these WTOs (Swain, Louttit, \& Hrudey). 
Provincial and municipal governments are becoming increasingly important secondary actors to the management of drinking water in First Nations communities (Auclair \& Simone, 2010). Federal departments have recently sought to create agreements and partnerships with provinces to enforce provincial guidelines for water treatment plants. Further, the AANDC Protocol for Centralised Drinking Water Systems in First Nations Communities stipulates that drinking water quality measurements should be in compliance with either the HC Procedure Manual for Safe Drinking Water in First Nations Communities South of $60^{\circ}$ or provincially set guidelines (INAC, 2010b, 2010c). The First Nation must adhere to the most stringent guideline, as provincial standards vary. This distinction is made so as to ensure that individuals living on reserves enjoy the same standards (or better) as other individuals within the same province. This caveat to the First Nation's water and waste water management plan has made it difficult to ensure that the appropriate guidelines are followed and provides space for confusion on what protocols should be followed by First Nations (Swain, Louttit, \& Hrudey, 2006). Increased provincial involvement has been contentious due concerns about the inherent right to self-govern (Wilson, 2004).

An important aspect of the four primary actors of drinking water management in First Nations communities for the purpose of this study is that the framework is not analogous to non-First Nations communities. Each of the three federal actors is independent, as each is directed by a distinct mandate. Further, EC and $\mathrm{HC}$ roles correspond specifically to their respective departmental mandates. AANDC's role is primarily to ensure that funding is disseminated to First Nations communities. The local First Nation governments are analogous to non-First Nations municipalities in that they 
are smaller bodies responsible for the day-to-day oversight of drinking water quality. However, First Nations governments are more reliant on public funding and management policies than non-First Nations communities. Further, First Nations governments enjoy higher political status than municipalities. That is, First Nations governments are akin to provincial governments in a federalism system. Specifically, elected band councils are delegated authority over First Nations reserve lands under the Indian Act, 1985 and are responsible for the public administration of their respective communities. Although the federal government enables the implementation and administration of First Nations, the federal government largely enables these governments autonomy significantly greater than that of municipalities. Indeed, the Federal government has a fiduciary obligation to consult with First Nations in the event that the federal government intends to make a decision that will impact the rights of First Nations peoples. Therefore, the simple twotiered drinking water management system prevalent in Ontario municipalities is not apparent in First Nations communities. Indeed, this lack of a common system has been regarded as contributing to poor drinking water quality on First Nations reserves (Graham, 2003; Swain, Louttit, \& Hrudey, 2006).

\section{Literature to Date}

Drinking water management in First Nations communities. There is a body of literature on the governance of First Nations' drinking water. Most literature was developed following the Walkerton crisis, which elicited national media attention to the management of drinking water in Canadian communities. As a result First Nations communities garnered exposure in the media. Since this time, a variety of perspectives have come to light regarding the causes of poor drinking water quality, including one 
such perspective which proposed that it is the independence of management responsibilities among a series of actors (i.e., AANDC, HC, EC, and the First Nations governments) that is contributing to drinking water quality issues (e.g., Graham, 2002; 2003). Some authors have suggested that a way to solve such differentiation is to allow First Nations self-governance overall (Peterson \& Torchia, 2008). Stemming from this literature is the notion that First Nations are better equipped to deal with their own issues in their own way (Wilson, 2004). Furthermore, it has been suggested that the separation of payment for water quality management from the responsibility for the actual management of drinking water quality has had negative ramifications for reserves (Smith et al., 2006). Specifically, this separation has forced First Nations to maintain responsibility of water treatment facilities built by AANDC which are only funded to $80 \%$ of the required operation and maintenance costs. The difference is expected to be made up by First Nations peoples, even though the communities often cannot afford it (Harden, Levalliant, 2008). It is suggested that First Nations who are provided adequate funding to self-govern, will be better able to treat and monitor their own drinking water sufficiently (Peterson \& Torchia).

Counter arguments to self-management illustrate case evidence that current First Nations self-governments that have difficulty managing water (Graham, 2002). For example, the Nisga'a First Nation in British Columbia was granted self-government, but has had difficulty operating and maintaining their drinking water system (Graham \& Fortier, 2006). Indeed, this community has been identified as high-risk for water contamination in the most recent national assessment of First Nations water systems (Neegan Burnside, 2011 a). Cases of successful self-governed water management, such as 
the Navajo in the United States, have demonstrated that there is a need for a large community population, multiple years of preparation, and a two-tier governance structure within a First Nation government (Graham \& Fortier, 2006). Specifically, the Navajo, a First Nation with a population of 140,000 , established its own Environmental Protection Agency (EPA). This EPA implements and enforces environmental laws and regulations including, but not limited to, drinking water quality within the First Nation land. The two-tier governance structure of these successful communities resembles the provincemunicipality relationship of drinking water management in Canada (Gram \& Fortier). Unfortunately, neither recommended conditions nor the capacity for these conditions are typically found in Canadian First Nation communities, as $61 \%$ of First Nations communities are populated by fewer than 500 people.

Another issue is that the determination of funding needs is not always clear. Specifically, the funding disseminated for operations and maintenance is based on AANDC projections rather than actual costs (Harden \& Levalliant, 2008). AANDC transfers funds specifically tied to infrastructure, operations and maintenance, and operator training to each First Nation community within its jurisdiction. Therefore, autonomy over drinking water related financials would allow First Nations' governments to allocate funds as necessary. The challenge is that First Nations reporting may be exaggerated to garner more funds.

A better alternative to self-government, or even a change in the management actors of First Nations community drinking water quality, is to create a more inclusive governance model for each First Nation community. It has been suggested that the issues persisting with regard to First Nations' water quality have been a result of independence 
among the four actors (Wilson, 2004). Indeed, Graham (2003) has notes that the longevity of such issues is not a matter of financial allocation, but rather divergent policy goals. It appears plausible that an inclusive management strategy would approach the issue in a coherent manner (Wilson). Wilson argues that integrated water management (including the overarching mandates of all four actors) is consistent with many First Nations philosophies. She further articulates that First Nations governments have been treated as stakeholders under the current management framework and recommends that, for an integrated management scheme to be established properly, ongoing and regular communication among the four actors is needed. Discussion and inclusion among the four actors is a repeated recommendation in the report of the expert panel on drinking water for First Nations, which suggests that the governance actors each bring value to the table with regard to their various areas of expertise, which should be utilized in developing a strong management strategy moving forward (Swain, Louttit, \& Hrudey, 2006).

Graham and Fortier (2006) have noted that there is a lack of clarity in the specific roles of among the four actors. They suggest that the lack of clarity has resulted in poor water quality. They articulate that there are poor regulatory systems, inadequate resource systems, and poor relationships among the four actors as a result of the independence. An inclusive management framework is a noted precondition for the success of future interventions. Interestingly, despite all the discussion surrounding inclusive management frameworks, no study to date has sought to explicitly illustrate the perspectives of the four actors. Therefore, a gap exists in the literature such that each actor's problem 
definition, change model, and theory of action for remedying the problem of First Nations' drinking water quality have yet to be fully investigated.

\section{Evaluations of drinking water quality of non-First Nations communities.}

Evaluations of drinking water systems in Canada culminate in a vast body of literature. The scope of these evaluations is similarly broad, having sought to assess several facets of drinking water quality. Most evaluations have examined rural communities and the role of agricultural farmland on community water usage (e.g., Coulibaly \& Rodriguez, 2003; Goss, Barry, \& Rudolf, 1998; Rudolph, Barry, \& Goss, 1998; Rudolf \& Goss, 1993). These evaluations have been conducted by academics in engineering and biology fields of study, where the primary focal point has been to elicit the level of chemical and microbiological contamination of drinking water. This type of evaluation is common because pathogenic contamination has been identified as posing the greatest recurring risk to the health of individuals consuming water from public sources (Hrudey, Huck, Payment, Gillham, and Hrudey, 2002). According to the World Health Organization, coliform bacteria are the most common microorganisms found in drinking water around the world (OMS, 1994; cited in Coulibaly \& Rodriguez). Canada has followed by testing primarily for levels of E. coli and total coliforms (e.g., Conboy \& Goss, 2007; Cool et al., $2010 ; 2011)$. Further, these tests of drinking water quality are of high importance based on their prevalence in Canada's Guidelines for Canadian Drinking Water Quality (HC, 2010a).

Canadians have not experienced equal access to safe drinking water. Specifically, the aforementioned evaluations on drinking water quality in Canada have illustrated a high level of contamination for rural communities' watersheds. For example, nearly $50 \%$ 
of 300 rural watersheds tested by Conboy and Goss (2007) had a higher number of total coliforms than acceptable under Ontario's drinking water regulations. Similarly, Goss and colleagues (1998) found that $40 \%$ of nearly 1,300 Ontario rural wells used for drinking water exceeded the maximum acceptable concentrations. Approximately, $34 \%$ of these water sources exceeded the standards of pathogenic water contamination. Other contaminants, such as a variety of chemicals utilized in agriculture, were also prevalent throughout the drinking water source sample. Such high levels of drinking water contamination are consistent with findings of other researchers (Hrudy, 2008; Petersen, n.d.; Rudolph \& Goss, 1993; Rudolph et al., 1998).

Reasons typically attributed to small and rural communities' difficulty with drinking water quality are the high costs of equipment; infrastructure in such communities is either obsolete or of low quality; local capacity to retain qualified or certified operators is low; and there is a lack of resources to properly fund water system operation and management (Auclair \& Simeone, 2010; Hrudy, 2008). These factors each relate to one overarching issue: small communities do not allocate sufficient financial resources for maintaining a drinking water system (Rollins et al., 1997). Graham (2003) indicates that communities must have a minimum of approximately 10,000 households to financially sustain acceptable drinking water quality. A second factor contributing to poor rural drinking water quality is location. Remote locations are commonly cited as a factor of quality drinking water due to costs for building and maintaining treatment systems (e.g., Hrudy; Petersen, n.d.). Another commonly cited factor is the lack of enforceable regulations (Isfeld, 2008). Together, these impediments to clean drinking water have placed small and rural communities at risk for water contamination. 
A second area of drinking water quality evaluations throughout Canada assesses the contamination risk of pathogenic microorganisms. For example, Cool and colleagues $(2010 ; 2011)$ assessed the vulnerability to contamination of 39 Quebec rural municipalities' drinking water systems, which illustrated that 21 (53.8\%) were highly vulnerable to microbiological contamination. However, the criteria for 'highvulnerability' include the risk of source contamination, treatment efficiency, distribution system, and local management of the water treatment plant. Of the criteria for vulnerability to contamination, only water source is not management related. Cool et. al.'s studies illustrate that surface water sources are more susceptible to contamination than are other sources (such as groundwater and groundwater under the influence of surface water). The differentiation in vulnerability is due partially to different management strategies for treating drinking water for each water source.

A third type of evaluation has sought to compare the water treatment of like drinking water systems. For example, Coulibaly and Rodriguez (2003) assessed the differences in ten rural Quebec drinking water systems, half of which were susceptible to contamination. The results from this study illustrated that the primary reason for recurrent contamination was the treatment process. Specifically, WTOs were not chlorinating the water effectively in either dose or duration. Further, the treated water did not maintain sufficient chlorine residuals to prevent future contaminations. Rural communities that were not susceptible to frequent contamination followed sound practices of dosage and residual maintenance. 


\section{Evaluations of drinking water quality of First Nations communities.}

Academic literature with regard to the evaluation of First Nations communities' drinking water is limited. The majority of such evaluations are qualitative in nature and describe the apparent disparity between First Nations communities and other Canadians. For example, Harden and Levalliant (2008) present qualitative descriptions of the drinking water quality within six First Nations communities. One such profile articulates that residents of the Kitigan-Zibi Anishnabeg community in Quebec are supplied with drinking water that is brown and has an odd smell. As a result, the band spends more than $\$ 200,000$ on bottled water each year. The profile also briefly describes the lives of two residents. One such resident attributes health problems (such as hair falling out) to the drinking water of the community, suggesting that the water source may be tainted with uranium, a known water contaminant in the region. Other qualitative evaluations have been devised by First Nations communities themselves, which have been presented in engagement sessions such as those of the Walkerton inquiry, the expert panel on drinking water for First Nations, and the Senate's drafting of Bills S-11, 2010 and S-8, 2012 (e.g., Neapetung, 2006; Kneeshaw, 2006; NAN, 2006).

Smith and colleagues (2006) conducted a risk assessment of First Nations communities' drinking water contamination in Alberta. Notably, these authors utilized the World Health Organizations drinking water quality guidelines in developing eight criteria for their evaluation. The eight indicators related to the following:

- Water source;

- Treatment process;

- Microorganism reduction;

- Reservoir and distribution;
- Finished water quality;

- Operation, maintenance and training;

- Monitoring and reporting; and

- Non-treatment infrastructure. 
These categories were individually weighted according to the evaluators' value judgements to assess overall risk for contamination. Overall, $50(89 \%)$ of the water systems evaluated were classified as high-risk, which occurred when the drinking water did not meet the maximum acceptable concentrations of potential contaminants within the federal drinking water quality guidelines; excessive or repetitive boil-water advisories were in place; or where significant changes to the management or operation of facilities were necessary. Most problems identified by the study were with regard to management operations, facility maintenance, and reporting compliance. The results of this study identified a much higher percentage of First Nations water systems in Alberta as highrisk (89\%) than did INAC in its 2003 national assessment (19\%).

Government conducted evaluations of drinking water quality. Governments have provided a second body of evaluations regarding drinking water quality. The majority of these have also focused on assessing risk for contamination. For example, the Ontario Ministry of the Environment (MOE) requires that all drinking water systems be assessed for contamination risk by independent engineers on a tri-annual basis (Holme, 2003). They assess a variety of factors related to risk including:

- Likelihood of hazardous events;

- A rank ordering of hazardous events by level of risk;

- Control measures related to contamination;

- Critical control points;

- Assessed currency and validity of reports; and

- Reliability and redundancy of water treatment equipment.

To date, approximately 18,000 drinking water systems have been assessed for risk in the province. Unfortunately, the results of these assessments have yet to be collated, as the MOE is still awaiting results from some regions (MOE, 2010). The reports of each Ontario community assessed are available to the public online (Holme). 
Similarly, AANDC has established risk assessment evaluations for First Nations communities. Three national risk evaluations have occurred in 1995,2001 and 2010 . The most recent risk evaluation was also conducted by an independent engineering firm and utilized more rigorous indicators than had previously been used by AANDC. Some regional risk assessments had included only First Nations self-assessments. This recent risk evaluation was based on five indicators of risk including:

- Water source;

- System design;

- System operation and maintenance;

- Operator training and certification; and

- Record keeping and reporting.

They were weighted according to AANDC's assessment of each indicator's severity of risk. Specifically, water source and record keeping and reporting were each weighted at $10 \%$; system design and system operation and maintenance were weighted at $30 \%$; and operator training and certification was weighted at $20 \%$ of the overall risk score. As noted, this more rigorous risk evaluation highlighted that 314 (39\%) First Nations communities' drinking water systems were high-risk for contamination. It is interesting to note that $21(26 \%)$ of Alberta's First Nations communities' drinking water systems were high-risk, an increase of $7 \%$ from the 2001 study.

An evaluation gap. The evaluation literature has yet to explicitly compare drinking water quality of First Nations communities with that of analogous non-First Nations communities. It is also significant to note that the evaluations of the two community types focus on different aspects of drinking water quality. Therefore, it is difficult to make explicit comparisons. Because risk evaluations by independent engineers have been solicited by governments, an opportunity exists to compare First 
Nations communities with non-First Nations communities while controlling for other known risk factors.

\section{Chapter by Chapter Summary}

The second chapter demonstrates the chosen methodology for addressing each subquestion. It delves into the specifics of the indicators and measures for these explorations and provides detail regarding the data sources utilized. The rationale for this chapter is to illustrate to the reader precisely which research techniques are used and to allow for future replication for further research and/or verification of empirical findings.

The third chapter delves into the different problem definitions and theories of change of the four management actors of drinking water of First Nations communities. The rationale for this chapter is to better articulate the roles and responsibilities of these actors, as such ambiguity has been identified as a factor which may contribute to the issue of poor drinking water quality in these communities (Graham \& Fortier, 2006).

Understanding these roles and responsibilities from the perspectives of the actors through publically available documents and interviews illustrates four distinct problem definitions and theories of change. Specifically, it was anticipated that EC's problem definition encompass issues pertaining to source water contamination; HC's problem definition illustrating that the problem has persisted because the treatment of drinking water has been lacking (e.g., lack of treatment facilities, lack of trained operators, chemicals not adequately dispensed, etc.); AANDC's problem definition focusing on the lack of a legislative framework for enforcing federal and provincial guidelines; and the First Nations' problem definition focusing on a lack of financial capacity to maintain quality drinking water. 
It is necessary to note that the problem definitions of AANDC and the First Nations were expected to be more comprehensive in scope, as these two actors are more intimately involved with the processes of drinking water management of First Nations communities. It was further anticipated that each actor's theory of change stems directly from its problem definition. Determining how and where these theories of changes diverge is an innovative approach to tackling the water management issues and provides a conceptualization on key areas where the four actors can better work together.

The fourth chapter compares the independent evaluations of drinking water quality and risk for contamination of the Ginoogaming First Nation with those of Longlac, Ontario. The rationale for including this chapter is that it serves as a control for the differences between the two comparisons that will follow. That is, the First Nations community is served by the non-First Nations community's drinking water system. The non-First Nation community is responsible for treating and disseminating drinking water to both its own community and the reserve community. The First Nation community is responsible for the distribution of drinking water throughout the reserve land. Drinking water quality and risk for contamination evaluations have been conducted both by independent engineers contracted by Longlac and AANDC. Therefore, differences between the evaluations illustrate whether problems are exaggerated or quelled either for the benefit of a community (for requesting funding allocations and/or meeting regulatory obligations) or due to flaws in the evaluation tools. This chapter thus provides both a third matched-case comparison lending to the thesis's argument and a series of caveats for the previous two chapters. 
The fifth chapter compares the water quality and risk for contamination of Sagamok Anishnawbek First Nation with Dowling, Ontario. These two communities are of similar population size and location. Both communities utilize groundwater as their drinking water source. The rationale for including this water source is that groundwater and surface water utilize different management and treatment techniques in their processes. This controlled comparison illustrates the First Nations community's drinking water quality as poorer and at higher risk for contamination in several areas relevant to the management of drinking water quality than that of Dowling. This hypothesis is consistent with water quality experts' indications that drinking water quality of First Nations communities is significantly poorer than that of non-First Nations communities (O'Connor, 2002b; Swain, Louttit, \& Hrudey, 2006). The chapter relates these differences specifically to the gaps identified between the governance actors' theories of change. Finally, the seeks to identify gaps in the management of drinking water only on the First Nations communities that illustrate areas not present within the four theories of change.

The sixth chapter compares the water quality and risk for contamination of Pikangikum First Nation with that of Red Lake, Ontario. These two communities are of similar population size, are in similar locations, and utilize a similar water source (surface water). It was anticipated that the results of this comparison would be similar to those of chapter four; however, it was assumed that differences between Pikangikum and Red Lake would be more pronounced as the management and treatment of surface water is more challenging than that of groundwater (Cool et al., 2010; 2011). This chapter also identifies key areas of risk for contamination that pertain to the management of drinking 
water, which are prevalent for the First Nation community only. These key areas are then related to the gaps prevalent throughout the four governance actors' theories of change.

The final chapter provides an overview of the findings in direct relation to the research question and sub-questions. The chapter also provides a thematic summary of findings from the three empirical chapters and relates these results to the gaps identified between the four governance actors' theories of change. As noted, it was anticipated that through these the matched-case comparisons the management gaps will be prevalent because of the independence of the four actors and the resultant divergence of the theories of change. Therefore, this chapter further explores these differences with evidence provided within chapters four through six and suggests specific areas where convergence of the four governance actors' change theories could benefit the management of drinking water quality of First Nations communities throughout Canada. This summary of findings is also augmented with national and Ontario-wide First Nations community data as well as Ontario non-First Nations drinking water quality data to provide a higher-level account of the major differences illustrated within the case studies. A series of recommendations are also offered to the reader on where to begin remedying the challenges of drinking water quality management in First Nations. Finally, the chapter includes a discussion the limitations and implications of the thesis as a whole. Overall, this thesis is exploratory in nature and thus provides a starting point for future research. 


\section{Chapter 2: Methodology for Addressing the Research Gaps}

In framing the thesis research question: "Does the governmental management framework surrounding drinking water systems on First Nations reserves contribute to the challenges for improving drinking water quality in these communities?," there are several subquestions that can be addressed. Two gaps have been identified in the literature, which require exploration before undertaking the major scope of the project. To address the first gap identified regarding the lack of clarity in the roles of the four main actors of drinking water management, two sub-questions are addressed:

(1) How do four federal and Aboriginal governance actors involved in managing drinking water on First Nations reserves define the problem regarding First Nations' drinking water quality? and,

(2) How do these actors' solutions for remedying the problem differ; and, in what specific ways do they diverge?

In order to address the second gap regarding a lack of evaluation research comparing First Nations and non-First Nations communities' drinking water quality matched-pair case studies were utilized. Through this direct comparison the thesis seeks to respond to the final sub-question:

(3) Are the differences in solutions for remedying drinking water quality issues on First Nations reserves contributing to the drinking water quality issues? This chapter illustrates, in detail, the methodology for addressing each of these subquestions. 


\section{Problem Definition}

To answer the first sub-question, two primary indicators were utilized for each of the four governance actors (see Table 1). The analysis of data obtained for the first indicator demonstrate the most common factors that each actor articulates as contributing to poor drinking water quality within the publically available information on their websites. To record factor references, the list of documents was reviewed, and detailed notes were made with regard to any phrases that the actor appeared to identify as a component to addressing drinking water quality (i.e., content analysis). Such phrases were identified when the documents articulated either that $\mathrm{XX}$ needed to be addressed or that $\mathrm{XX}$ was resulting in drinking water quality issues. The subject of each of these phrases was then identified as specific terms. Using the 'find' function in Microsoft Word, each document was reviewed to identify the number of times a root term was used (e.g., treat, treatment, treating) in the proper context. The total number of terms for a given governance actor was expressed as the total number of 'factor references' (see Annex 2). Within chapter three, terms are presented as the number of times the governance actor uses the term as a percentage of all factor reference terms used (see Annex 3). Reference lists for the documents reviewed are included in Annexes 4-7. The analysis of data obtained for the second indicator demonstrate the factors that interview respondents of each actor articulate as most prevalent in contributing to poor quality of drinking water of First Nations communities. These 'factors' include elements of a drinking water system that need to be addressed according to interview respondents. Interview respondents were identified from referrals to senior officials within each organization who have significant knowledge and understanding of First Nations communities' drinking water quality 
issues, and their respective organizations' approaches for addressing the issues. Profiles of the interview respondents are presented in Annex $\mathbf{8}$ and interview questions used are provided in Annex 9.

Individual First Nations community governments are each likely to be distinct in their specific problem definitions and theories for addressing First Nations drinking water quality issues, As such, leading First Nations organizations are analyzed instead. The data source for the first indicator of the problem definition includes all documentation pertaining to drinking water on the Assembly of First Nations (AFN) and Chiefs of Ontario $(\mathrm{CoO})$ websites. The rationale for using these organizations for analysis was that each of the First Nations selected as case studies, in conjunction with most First Nations communities, chose the leaders of these organizations to represent their political interests. These groups represent the generally held First Nations positions on federal drinking water quality policies. The AFN is a leading national organization First Nations organization. The $\mathrm{CoO}$ is the representative body for First Nations communities in Ontario. 
Table 1: Problem Definitions Indicators and Measures

\begin{tabular}{|c|c|c|}
\hline \multicolumn{2}{|r|}{ Indicator } & Measures \\
\hline a) & $\begin{array}{l}\text { Alleged factors contributing to } \\
\text { poor drinking water quality in } \\
\text { First Nations communities } \\
\text { made publically apparent }\end{array}$ & $\begin{array}{l}\text { - Number and type of factors alleged to contribute to the } \\
\text { poor quality of drinking water of First Nations } \\
\text { communities in publically available documents } \\
\text { - Frequency of alleged contributing factors }\end{array}$ \\
\hline b) & $\begin{array}{l}\text { Alleged factors contributing to } \\
\text { poor drinking water quality of } \\
\text { First Nations communities not } \\
\text { publically available }\end{array}$ & $\begin{array}{l}\text { Number and type of factors government actor interview } \\
\text { respondents allege as contributing to the poor quality of } \\
\text { drinking water of First Nations communities } \\
\text { - Rank ordering of alleged contributing factors }\end{array}$ \\
\hline
\end{tabular}

Together, the two indicators assist to triangulate the specific problem definition of each organization. Moreover, the most prevalent factors illustrate those issues to which the governance actors most attribute the problem. Analysis of the specific measures also illustrates precisely where problem attributions diverge among the four management actors.

\section{Theories of Change}

Addressing the second sub-question requires more in-depth indicators (see Table 2). A theory of change has been defined simply as how and why an initiative works (Weiss, 1995). It is a series of interventions and/or activities designed to address a particular issue. In the case of First Nations drinking water systems, it refers to each actor's overarching ideal strategy for addressing the identified drinking water problem. A theory of change is articulated through a change model. A change model is the ideal map of specific interventions and activities for addressing the overarching problem of drinking water quality, which each has previously defined. According to Connell and Kubisch (1998), the activities that an organization implements to bring about desired outcomes must be analyzed to understand that organization's theory of change. 
The theory of change and change model approaches were chosen as opposed to more traditional forms of analysing government intervention (i.e., policy instruments and policy goals), as this approach goes beyond these models in tracing back the logic as to how and why a series of interventions will work. The change models are used to flesh out precisely how each organization ideally sees the different interventions interacting to result in the desired outcome. These tools are different from policy instrument and policy goal analysis, as they seek to get to the crux of how and why the intervention will work and whether or not the logic is sound for producing the desired effects. Therefore, several indicators with regard to the specific activities and desired outcomes of each actor are analyzed using the W.K. Kellogg Foundation's Logic Model Development Guide (2004) and Mayne's (2008) contribution analysis technique for exploring cause-and-effect.

\section{Table 2: Theories of Change Indicators and Measures}

\begin{tabular}{|c|c|c|}
\hline \multicolumn{2}{|c|}{ Indicator } & Measures \\
\hline a) & Adopted management strategy & - Number and type of activities to be completed \\
\hline b) & $\begin{array}{l}\text { Temporal prioritization of } \\
\text { activities required in the } \\
\text { change strategy }\end{array}$ & $\begin{array}{l}\text { - Number of times activities articulated } \\
\text { - Rank ordering of activities by interview respondent(s) }\end{array}$ \\
\hline c) & $\begin{array}{l}\text { Desired outcomes of the } \\
\text { change strategy }\end{array}$ & $\begin{array}{l}\text { - Number of outcomes to be reached } \\
\text { - Type of outcomes to be reached } \\
\text { - Goals of the change theory }\end{array}$ \\
\hline d) & $\begin{array}{l}\text { Rationale for why the change } \\
\text { strategy will attain desired } \\
\text { outcomes }\end{array}$ & $\begin{array}{l}\text { - Qualitative descriptions linking activities from the first } \\
\text { and second indicators to outcomes of third indicator }\end{array}$ \\
\hline e) & $\begin{array}{l}\text { Assumptions used to validate } \\
\text { the change strategy }\end{array}$ & $\begin{array}{l}\text { - Resources required to implement desired activities } \\
\text { - Time-frame for achieving desired outcomes } \\
\text { - Activities required by other management actors }\end{array}$ \\
\hline
\end{tabular}

The analysis of data obtained for the first indicator demonstrate the most common activities that each actor depicts as necessary for improving First Nations drinking water quality (see Annexes 2-3), while the data analysis of the second provides an order of 
immediacy for the activities. Data for the third and fourth indicators illustrate the specific desired outcomes and goals of each intervention of each actor's change theory as well as the rationale as to why the chosen activities result in the desired outcomes and goals. Data obtained from the final indicator is used to articulate assumptions that are used as a rationale for the results of the fourth indicator. Two data sources for the five indicators are utilized: web pages and documents, semi-structured interviews. With respect to the former, all documents were publically available on the websites of the five organizations (see Annexes 4-7). Interview respondents were asked questions regarding their respective organizations' theory of change (see Annex 8).

In order to develop the theories of change and change models, understanding context was imperative to form a contribution story. That is, each step was identified as a contributing component to addressing the overall challenge of drinking water quality in First Nations communities. First, document reviews and interviews with key government officials were conducted to identify the most frequent factor references that contribute to drinking water quality. From this list the terms were relayed into specific activities to address these factors. A combination of the number of times each activity was articulated and the prioritization of activities by interview respondents informed the initial drafting of the order of logic models. As articulated within the documents and by the interview respondent, the desired outcomes, type of outcomes to be reached, and goals of the change theory were then reviewed to provide stronger logic to the change theory, resulting in a re-ordering of specific steps. Rationales for why change strategies will attain the desired outcomes were then developed to further inform the ordering of specific steps and were utilized to establish feedback loops. Assumptions used to validate the 
change strategy, typically garnered from program mandates, were also considered to contribute to feedback loops. To provide a more in-depth analysis of each step of each actor's theory of change and subsequent change model, theories of action are also developed. A theory of action (or action theory) regards precisely how a set of program or intervention activities are devised by the program planners, decision-makers, and or implementers (Chen, 1990). It can encompass the details of an intervention including each of the following: the implementing organization(s), individual implementer(s), associate organizations and community partners, the ecological context of the intervention, intervention and service delivery protocols, and target population(s) (Chen, 2005). It also provides space to demonstrate the resource inputs into the intervention. Annex 10 illustrates Chen's action theory model and how it is able to integrate with the broader change model (or theory of change). There is an action theory for each step of each actor's change model. Action theories are explored utilizing information obtained primarily from the third indicator, and are included as figures for providing greater depth to each step of the four actors' change models. Action theories are informed by the same data sources as the indicators.

\section{Comparing First Nations Communities with Analogous Non-First Nations}

\section{Communities}

To address the second gap, a third sub-question is explored: "Are the differences in solutions for remedying drinking water quality issues on First Nations reserves contributing to the drinking water quality issues?"

Three matched-pairs case studies involving the comparison of First Nations communities with analogous non-First Nations communities were utilized to address this 
question. A sample of First Nations communities was paired with non-First Nations communities by population size, location, and water source. Each of these three characteristics was commonly noted as a factor contributing to the risk of drinking water contamination. The rationale for pairing First Nations communities with non-First Nations communities is that it enables a comparison of a provincial drinking water management framework (i.e., the two-tiered approach) with that of First Nations communities (i.e., the four actor approach) such that differences in management could be readily identified.

Case selection. Utilizing multiple case study pairs enables the control for nongovernance factors. Three case study pairs were selected according to water source, as risk for contamination increases along with the continuum of water source, increasing from Municipal Type agreement, groundwater, groundwater under the influence of surface water, to surface water (Cool et al., 2010, 2011; Neegan Burnside, 2011a). The rationale for selecting on the basis of water source is that these require distinct management techniques in source protection and treatment (Cool et al., 2010; 2011). Further, the majority of First Nations are small and located in northern and often secluded parts of Canada (Neegan Burnside). Therefore, each water source illustrates whether the management frameworks for drinking water of First Nations communities is more adept at ensuring quality drinking water with one water source over another. Additionally, the comparison between the First Nations and non-First Nations communities illustrates whether the First Nations management framework is able to produce drinking water quality analogous to that of non-First Nations communities based on different governance frameworks as a control factor. 
Only communities that are served by one water source were selected for this study to ensure that the results illustrate the effects of the management frameworks on one specific water source in a given region. It is necessary to note that a Municipal Type Agreement (MTA) is negotiated between a First Nation and a nearby municipality. This matched-case pair is critical to the thesis because it demonstrates differences precisely where measures of risk diverge: an issue between risk assessments illustrated earlier between Smith et. al.'s (2006) study of risk on Alberta based First Nations communities and AANDC's assessment of those communities.

The water source "groundwater under the direct influence of surface water" was not included as a matched-case pair. This decision was made for two important reasons: there are few Ontario First Nations communities using this source (only 11); and of the few communities utilizing this source, none are near non-First Nations communities utilizing this source.

Ontario's water management framework is regarded as the most rigorous in Canada (Holme, 2003). Therefore, only communities in Ontario were selected as matched-pairs because regulations were assumed to be consistently applied across all regions. Such consistency is not the case with the management of other provinces (Isfeld, 2009).

Case selections: Municipal type agreement. The case selection for a First Nation utilizing an MTA as its water source is Ginoogaming First Nation, which has an agreement with Longlac. Ginoogaming has a population of 167, whereas Longlac's population is 1,388 . Longlac is located 4.5 kilometres west of Ginoogaming (Highway 11, 2011). 
Case selections: Groundwater source. The selection for a First Nation is Sagamok Anishnawbek First Nation. This community is home to 1,036 individuals and is located near Lake Huron (Statistics Canada, 2012a). The town of Dowling Ontario also utilizes groundwater as its drinking water source with a population of 1,690 individuals (Statistics Canada, 2012b), and is located approximately 60 kilometres away from Sagamok Anishnabek.

Case selections: Surface water source. The selection for a First Nation community utilizing surface water as its primary source for drinking water is Pikangikum First Nation, which is located in Northwestern Ontario and has been noted as having some of Canada's worst drinking water quality conditions (Harden \& Levalliant, 2008; IFNA, 2000). The community of 2,100 people obtains its drinking water from Pikangikum Lake on the Berens River (Statistics Canada, 2012e). This community is a large First Nation located near Ontario's most northwestern municipality, Red Lake Ontario. Red Lake's 1,274 residents obtain drinking water from Red Lake (Statistics Canada, 2012d). The community is located approximately 95 kilometres south of Pikangikum.

Indicators and measures. Several indicators were selected to determine whether the differences between the four actor's theories of change pertaining to drinking water quality are contributing to the water issues faced by these First Nations communities (see Table 3). Most of these indicators have been adapted from the measures used by the Protocol for Centralised Drinking Water Systems in First Nations Communities (the Protocol) (INAC, 2010b) and the Ontario Ministry of the Environment's (MOE) requirements for water system risk assessments as stipulated in the Safe Drinking Water 
Act, 2002, Ontario Regulation 170/03. Data obtained from the first indicator demonstrate the required number of personnel and certification training required by the Protocol and the Safe Drinking Water Act, 2002, as well as each community's actual personnel and certification levels. Results obtained from the second through fifth indicators demonstrate each community's level of compliance with the Protocol/Safe Drinking Water Act, 2002 for various elements of the drinking water system. Analysis of final indicator data depicts the gaps each community must fill in order to fully comply with the Protocol/Safe Drinking Water Act, 2002.

Data sources for these indicators include individual community risk evaluation reports by independent engineers submitted to AANDC and the Ontario MOE. Data for the indicator e) was also drawn significantly from annual community reports submitted to the MOE regarding drinking water quality testing (non-First Nations communities) as well as $\mathrm{HC}$ reports on drinking water quality (First Nations communities). Data was also supplemented by information found on community, federal department, and provincial department websites, as well as published peer-reviewed journal articles where available. Finally, semi-structured interviews with key interview respondents and senior public officials were also used to supplement the primary data sources. See Annex 8 for a general profile of the interview respondents that agreed to be quoted in the thesis. 
Table 3: Matched-Pair Case Indicators and Measures

\begin{tabular}{l} 
Indicator \\
\hline a) A sufficient number of staff are \\
available and appropriately \\
trained for the community
\end{tabular}

b) The drinking water system meets current design requirements (INAC, 2010b; Ontario, 2002)

c) The water source is adequately protected from contamination

d) The operation and maintenance practices of the treatment system are compliant with regulations/guidelines

e) The current level of drinking water quality of the case study communities is identified

f) Required improvements of the drinking water system
Measures

- Number of and position of individuals involved in the management of drinking water

- Required certification level of the system (INAC, 2010b; Ontario, 2002)

- Certification level each WTO has attained

- Number of years experience of each WTO

- Whether a disinfection system is in place

- Whether appropriate treatment processes are followed (specific to the water source)

- Whether the system is reliable;

- Whether the system has a flexible design

- Whether the community's water needs are less than $75 \%$ of the capacity of the system

- Whether the water system has a source water protection plan (INAC, 2010b; Ontario, 2002)

- The number and type of protocols for addressing a contamination of the water source

- Additional activities undertaken to protect source water

- Amount allocated for operations and maintenance costs

- Whether funds for maintenance have been used for operations and maintenance

- Amount of funds needed for further maintenance

- Operations and maintenance activities that are currently in place (INAC, 2010b; Ontario, 2002)

- Whether operation and maintenance manuals and schedules are in place (INAC, 2010b; Ontario, 2002)

- Water quality parameters reported to $\mathrm{HC}$ and the MOE

- The number, type, and frequency of monitoring practices (INAC, 2010b; Ontario, 2002)

- Number and type of improvement recommendations

- Percentage of recommended improvements relating to each of the other indicators

- Estimated cost of these improvements by category (based on the above indicators)

- Estimated cost percentage of recommended improvements relating to each of the other indicators 


\section{Chapter 3: First Nations Governance Actors' Diverse Theories for Remedying Drinking Water Quality Issues}

The purpose of this chapter is to demonstrate the specific areas of divergence between the four governance actors, as these are alleged to contribute to First Nations drinking water quality issues. Such analysis provides a foundation on which to compare First Nations and non-First Nations drinking water quality. It illustrates whether or not the differences between communities governance structures indicate the situation is less apt for managing drinking water quality in First Nations relative to non-First Nations, as articulated by many drinking water quality experts in Canada (O'Connor, 2002b; SDWF, 2011; Swain, Louttit, \& Hrudey, 2006).

As indicated, the four principal actors include Aboriginal and Northern Development Canada (AANDC), Health Canada (HC), Environment Canada, and First Nations (herein represented by Assembly of First Nations (AFN) and Chiefs of Ontario (CoO). Swain, Louttit, and Hrudy (2006) argue that the independence and diversity with which these actors approach problems pertaining to First Nations drinking water has contributed to the persistence of various drinking water problems.

In the sections that follow each of the four governance actors' problem definitions and change theories are described and compared.

\section{Aboriginal Affairs and Northern Development Canada}

A total of 48 publically available documents were reviewed and analyzed for content from AANDC's website to develop the problem definition and theory of change.

Documents reviewed were selected from several facets of the department's work on First Nations drinking water quality. Specifically, these documents focused on the 
department's First Nations Water Management Strategy (FNWMS), the Plan of Action for Drinking Water in First Nations Communities, the First Nations Water and Wastewater Action Plan (FNWWAP) (formerly First Nations Water Action Plan), and the externally contracted National Assessment of First Nations Water and Wastewater Systems. These documents typically focused on the amount of investment the department has made to date. Other documents establish specific protocols developed to ensure quality drinking water in First Nations communities, such as the Protocol for Centralised Drinking Water Systems in First Nations Communities (the Protocol). Senior public officials at AANDC were also interviewed and served as supplemental data sources for this section.

AANDC's approach for addressing drinking water quality issues of First Nations communities is principally regulation and compliance. AANDC's rationale is that enforcing compliance will pressure First Nations communities to fulfill their obligations. Presumably, enforcing consistent management will ensure consistent contamination risk across all First Nations and will result in similar contamination risk to that of analogous non-First Nations communities. An interesting element of AANDC's change theory is the degree of participation by First Nations' leadership. The department consults with First Nations at selected points in the setting of policy, enforcement, and compliance measures. The department's language appears to indicate that its work will build First Nations capacity, as opposed to assisting First Nations with building such capacity. Despite this language, the long-term theory of change would indicate that it wishes to promote capacity in First Nations communities to maintain drinking water quality. 
Problem definition. AANDC defines the problem of drinking water quality in First Nations as a direct relationship to contamination risk. The department measures the likelihood a water system will be contaminated. AANDC developed three national risk assessments of drinking water systems in First Nations communities in 1995, 2001, and 2011 , which illustrated an increase in the number of First Nations communities at high risk for contamination, partially due to an enhancement of the measures and data acquisition methodologies. The most recent assessment categorized 39\% (314) of First Nations communities as high risk, meaning that immediate corrective action is needed for one or more major deficiencies to address either health and safety or environmental concerns (Neegan Burnside, 2011a). Further, 34\% (278) of communities were categorized as medium risk, meaning that corrective action is warranted but not immediately required for one or more system deficiencies in order to address either health, safety, or environmental concerns (Neegan Burnside, 2011a). These risk ratings illustrate the number and type of drinking water system deficiencies prevalent within First Nations communities. AANDC includes five such types of deficit related contamination risk. These deficiencies represent the primary components of the problem definition pertaining to drinking water quality as defined by AANDC.

First, the source of water (4.8\% of AANDC factor references) significantly contributes to the potential for contamination (surface water is more at risk than groundwater). Also, the quantity available for consumption is related directly to water source. Second, the design of the water system (3.7\% of factor references) determines the manner in which water is treated. Third, system operation ( $4.8 \%$ of factor references) and maintenance $(5.2 \%)$ practices affect the continued treatment and longevity of water 
systems. Fourth, Water Treatment Operator (WTO) training (4.9\% of factor references) and certification $(2.3 \%)$ contribute to the capacity for operations and maintenance (O\&M). Finally, record keeping ( $0.2 \%$ of factor references) and reporting $(0.6 \%)$ factor into contamination and drinking water events (DWEs). In 2006 AANDC placed value ratings on each factor, establishing those factors that contribute most to drinking water contamination risk. The risk factors are as follows:

- Water source $(10 \%)$,

- System Design (30\%),

- Operations and Maintenance (30\%),

- Training and Certification (20\%), and

- Reporting (10\%).

Factors relating to the operation and maintenance are the primary issues with drinking water quality in First Nations. For example, if WTOs are properly trained and certified then they will be able to operate and maintain the drinking water system and should be prepared to comply with reporting requirements. The following quotation best summarizes the department's problem definition:

While funding for capital projects may seem like the most obvious solution, design risks only account for 30 per cent of the risk identified. Operation and maintenance, operator qualification, and record keeping account for 60 per cent of the risk measured. This highlights that although design and construction are an important cost, once a system is built; ensuring that it continues to produce safe water for a community is dependent on the people operating the system (AANDC, 2011d).

Interview respondents indicated that the operation and maintenance of water systems is a primary factor for drinking water quality issues in First Nations communities. The need for capable, trained WTOs was identified as the most important factor. As one interview respondent stated, "most people would say 'I'll take a mediocre plant with an excellent operator over a great plant with a poor operator"' (personal communication, March 27, 2012). 
Theory of change. AANDC's stated vision is "a future in which First Nations, Inuit, Métis and northern communities are healthy, safe, self-sufficient and prosperous" (AANDC, 2012a). The department's objectives in this regard are to support First Nations to "improve social well-being and economic prosperity; develop healthier, more sustainable communities; and participate more fully in Canada's political, social and economic development" (2012a). According to the analysis by the Expert Panel on First Nations Drinking Water, AANDC is responsible for a range of activities including: $100 \%$ of funding for infrastructure development related to drinking water, $100 \%$ of funding for First Nations' WTO training; $80 \%$ of funding for O\&M of First Nations water systems; and enforcing standards per funding agreements with First Nations communities (Swain, Loutit, \& Hrudey, 2006). Together these objectives make it apparent that the departmental change theory is to shift the current status quo of First Nations communities from one that is dependent on the federal government to one that is self-sustaining and capable of maintaining health standards and economic prosperity.

With regard to drinking water in First Nations communities, AANDC's mandate includes: investments in infrastructure; enforceable standards and protocols; proposed legislation; and capacity and training. These broad mandates directly align with those responsibilities the expert panel (Swain, Loutit, \& Hrudey, 2006) identified. As such, AANDC's change theory and mandate places the onus of ensuring that water systems are efficiently funded, operated, and maintained on First Nations communities.

AANDC's change theory is developed with several activities in mind, including: infrastructure investment; regulatory enforcement; engagement with First Nations; education and awareness of drinking water quality issues; and clear roles and 
responsibilities of all actors. With respect to the first activity, AANDC has indicated that its investment portfolio focuses first on communities deemed high-risk by national assessments. The department addresses all high-risk infrastructure issues and then focuses on medium- and low-risk issues once high-level risks are mitigated. Second, a summative evaluation of the FNWMS and FNWWAP illustrated that operations procedures set out in the AANDC protocols were not typically followed and that "in some cases bands did not budget for operations and maintenance" (INAC \& HC, 2007). Interview respondents noted that First Nations rarely cover the $20 \%$ of O\&M funding for which they are responsible. The evaluation concluded that enforcing compliance through funding agreements has had limited success, warranting the enforcement of drinking water quality regulations. Third, First Nations engagement is important to the success of developing and implementing regulations. Figure 1 illustrates AANDC's proposed process for developing regulations, which includes information sharing and engagement sessions in only two of six stages. Fourth, AANDC's change theory suggests that there is need for greater education and awareness on the part of all actors with regard to the importance of drinking water quality. Such education and awareness is directed at First Nations residents, children, and other target groups. Governance actors need to better understand their roles, responsibilities, and the importance of their work, and residents need to better understand how they can contribute to protecting source water. 
Figure 1. AANDC Proposed Legislative Process

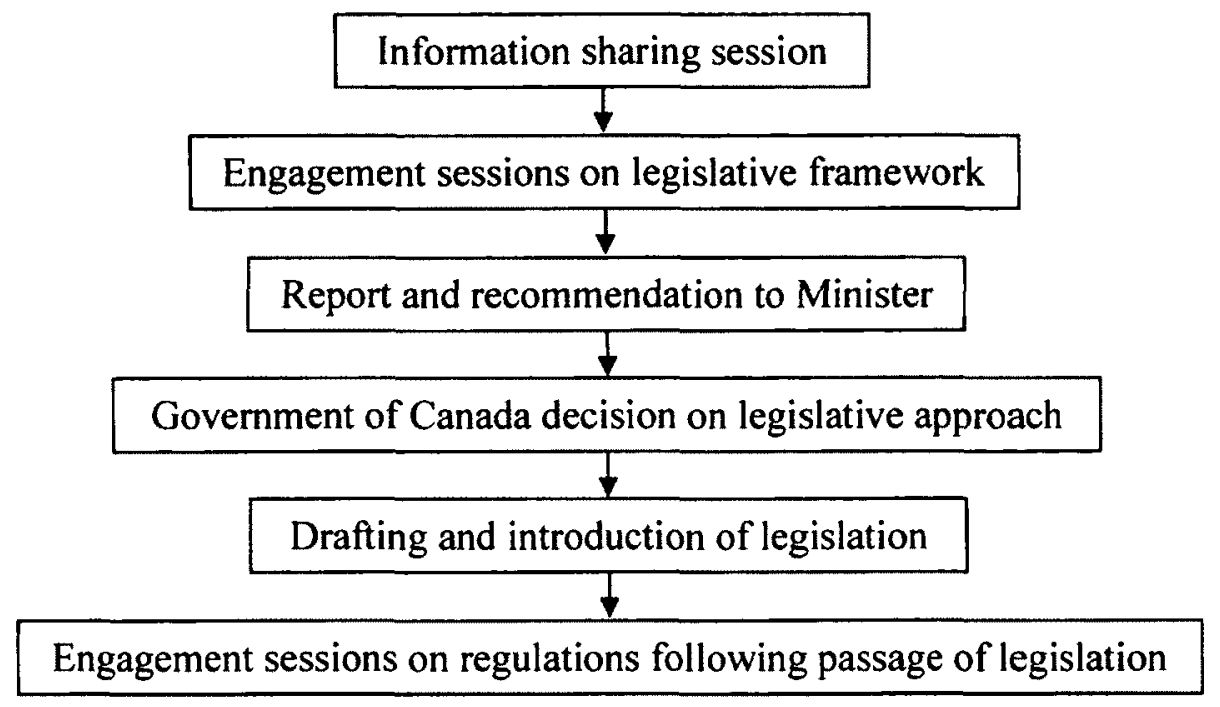

Change model. AANDC's change model is related to the department's problem definition and theory of change. It comprises the nine sequential steps contained in Figure 2. It is important to note that solid arrows identify direct pathways and dotted arrows illustrate feedback loops.

Figure 2. AANDC's Change Model

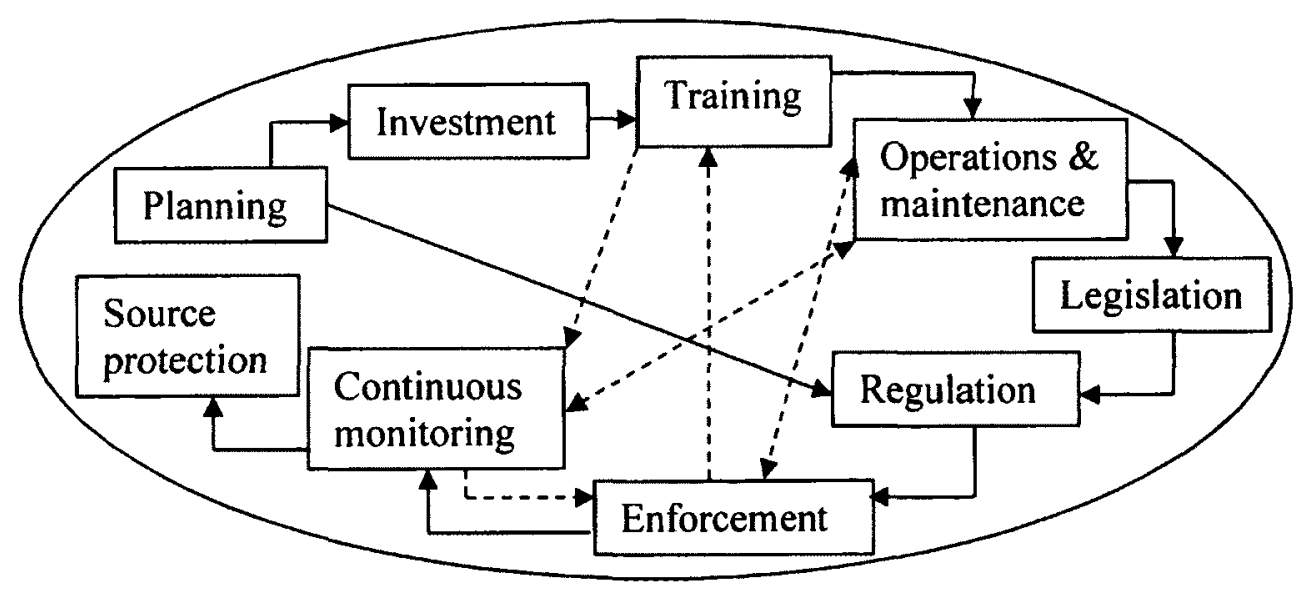


The first step in establishing a plan of action for remedying underlying issues was planning ( $6.2 \%$ of factor references). This step was cited throughout the documents reviewed. AANDC notes the need to develop remedial plans for each individual First Nation community's drinking water system. The foundation for such planning is founded on previous assessments ( $4.2 \%$ of factor references) and evaluations $(0.7 \%)$. In fact, the national risk assessment is a tool for illustrating risk variables most prevalent in First Nations communities. Table 4 summarizes the action theory for this step according to AANDC.

Table 4. AANDC Action Theory: Planning

\begin{tabular}{ll}
\hline Action Theory Component & Required Inputs \\
\hline a) Resources & $\begin{array}{l}\text { Unspecified amount from } \$ 330 \mathrm{M} \text { biannual A-Base } \\
\text { allocations from 2008-13, \$165M from Canada's Economic }\end{array}$ \\
& Action Plan, and \$600M for FNWMS from 2003-08 \\
b) Implementing Organizations & - AANDC, HC, EC, Chief and Councils (C\&Cs) \\
c) Implementers & - Policy drafters \\
d) Associates/Partners & - C\&Cs, experts ${ }^{2}$, First Nations groups (e.g., AFN, CoO) \\
e) Ecological Context & - Limited budget, more than $\$ 1$ B in investments still required \\
f) Intervention Protocols & - The Protocol, expert reports ${ }^{3}$, assessments, evaluations \\
g) Target Population & - First Nations community residents
\end{tabular}

Once a plan has been set in place, it follows that the plan be implemented beginning with the supply of needed facilities. Approximately $19.5 \%$ (158) of all First Nations water systems make direct use of raw water without the use of an operational treatment plant (Neegan Burnside, 2011a). Further, the majority of First Nations community water systems require infrastructure-related upgrades to meet the Protocol. Such upgrades include new or "additional disinfection facilities, additional chlorine

\footnotetext{
${ }^{2}$ Experts include the Expert Panel on Drinking Water for First Nations and the Standing Senate Committee on Aboriginal Peoples among others

${ }^{3}$ Including the Report of the Expert Panel on Safe Drinking Water for First Nations, the Standing Senate Committee's final report on Safe Drinking Water for First Nations, and the Neegan Burnside (2011b) report
} 
contact time, the provision of standby power, improvements to chemical storage facilities and the provision of an additional well, or an additional treatment train" (Neegan Burnside, 2011a). AANDC's change theory suggests that the department recognizes the need for infrastructure investment in treatment plants, system piping, and other capital costs in providing quality drinking water as stated in the Protocol. As noted above, terms pertaining to system design were prevalent ( $3.7 \%$ of factor references). Terms such as infrastructure (2.2\% of factor references) and investment (3.7\%) were cited throughout the review respectively. It should be noted here that the cost of infrastructure investments are exceedingly high, estimated at approximately $\$ 1.1$ billion across Canada as of 2011. In line with the overall change theory, $89.4 \%$ of these costs are associated with the 592 (73.4\%) high- and medium-risk First Nations water systems. By 2010 AANDC spent more than $\$ 820$ million on infrastructure related to water treatment; however, it is evident that more investment is required. Table 5 summarizes the action theory of the investment intervention according to AANDC. 
Table 5. AANDC Action Theory: Investment

\begin{tabular}{ll}
\hline Action Theory Component & Required Inputs \\
\hline a) Resources & $\begin{array}{l}\text { - } \$ 820 \mathrm{M} \text { from investments between 2006-2010, unspecified } \\
\text { amount from } \$ 330 \mathrm{M} \text { biannual A-Base allocations from }\end{array}$ \\
& $2008-13, \$ 165 \mathrm{M}$ from Canada's Economic Action Plan \\
b) Implementing Organizations & - AANDC, HC, First Nations, contracting organizations \\
c) Implementers & - AANDC policy drafters, C\&Cs, contractors \\
d) Associates/Partners & - HC, Public Works and Government Services Canada \\
& (PWGSC), Canada Mortgage and Housing Corporation \\
e) Ecological Context & - Limited budget, more than $\$ 1 B$ in investments still required \\
f) Intervention Protocols & - The Protocol \\
g) Target Population & - First Nations community residents
\end{tabular}

It follows that once water systems with adequate equipment are in place, trained personnel are necessary to operate them effectively. WTO training ( $4.9 \%$ of factor references) was noted frequently in AANDC documents and interviews. The theory is that increased training will improve the management of water systems, which will help "ensure standards for health and safety are met; increase reliability of systems; ensure efficient operation; decrease the number of drinking water advisories; [and] improve the life span of infrastructure" (AANDC, 2011e). AANDC also seeks to train WTOs to the point of certification ( $2.3 \%$ of factor references) to be sure that individual operators have been provided with the appropriate instruction for operating and maintaining water systems ${ }^{4}$. The department notes that only eight percent of First Nations WTOs possessed

\footnotetext{
${ }^{4}$ WTO training occurs primarily through the Circuit Rider Training Program and is delivered by "a variety of partners and service providers including private companies, tribal councils, and First Nation technical organizations" (AANDC, 2011e). WTO training occurs in four 'levels' for treatment and four 'levels' for distribution. The required level of training is directly associated with the classification levels of the treatment and distribution systems (i.e., a Level I treatment system requires Level I treatment certification and a Level II distribution system requires a Level II distribution certification.
} 
any level of certification prior to $2002^{5}$. Currently, only $60 \%$ of First Nations WTOs have been trained to the first level of certification (AANDC, 2011e). These figures are striking considering that interview respondents indicated that "human component to treatment is key to the success of drinking water systems" (personal communication, March 27, 2012). Table 6 summarizes the AANDC's action theory for training First Nations WTOs.

Table 6. AANDC Action Theory: Training

\begin{tabular}{ll}
\hline Action Theory Component & Required Inputs \\
\hline a) Resources & - Approximately \$10M per year \\
b) Implementing Organizations & - Circuit Rider Training Organizations (including Provinces) \\
c) Implementers & - Circuit Rider Trainers \\
d) Associates/Partners & - C\&Cs, AANDC, HC, treatment and training organizations \\
e) Ecological Context & - $40 \%$ of First Nations WTOs hold no certification \\
f) Intervention Protocols & - Circuit Rider Training Program, the Protocol, GCDWQ \\
g) Target Population & - WTOs
\end{tabular}

If WTOs are adequately trained they will be able to operate systems appropriately, using standard O\&M practices. Facility operation (4.8\% of factor references) and maintenance (5.2\%) in compliance with departmentally prescribed protocols $(4.6 \%)$ was identified as a crucial component in the change theory. Specifically, water treatment can commence by following the "comprehensive, scientifically defensible, and achievable performance standards" as per the Protocol (INAC, 2006). The treatment (5.9\% of factor references) and disinfection (2.1\%) component of operations is important for quality drinking water delivery. However, the AANDC interview respondents suggested that lacking water system O\&M in First Nations communities has hampered the treatment process due to unprecedented deterioration of the treatment

\footnotetext{
${ }^{5}$ WTO certification is a listed requirement of the AANDC Protocol for Safe Drinking Water in First Nations Communities; however, as noted above, such provisions are treated as guidelines for First Nations communities.
} 
systems (personal communication, March 27, 2012). AANDC's action theory for O\&M is summarized in Table 7.

Table 7. AANDC Action Theory: Operations and Maintenance Action Theory Component Required Inputs

a) Resources

- $80 \%$ funded by AANDC, $20 \%$ by First Nations; $\$ 431 \mathrm{M}$ between 2006-10, unspecified amount from $\$ 330 \mathrm{M}$ from biannual A-Base allocations from 2008-13, $\$ 40 \mathrm{M}$ in additional departmental allocations per year

b) Implementing Organizations

- First Nations

c) Implementers

- WTOs

d) Associates/Partners

- C\&Cs, Circuit Rider Trainers, HC, AANDC

e) Ecological Context

- Funds are not always allocated appropriately by $C \& C s^{6}$

f) Intervention Protocols

- The Protocol, GCDWQ, provincial legislation

g) Target Population

- First Nations community residents

Another step in the change theory, which is part of WTO compliance with AANDC protocols, is the development of a legislative framework. Legislation is necessary to enable enforceable regulations across all non-self-governing First Nations and those self-governing First Nations that wish to opt-in (AANDC, 2012b). Legislation ( $2.6 \%$ of factor references) in this sense was cited frequently throughout the AANDC documents reviewed as an important instrument for change. Indeed, two legislative bills have been read in parliament including Bill S-11: The Safe Drinking Water for First Nations Act, which died on the Order Paper in March 2011. Bill S-8: The Safe Drinking Water for First Nations Act is similar to Bill S-11, with a few caveats regarding the Government of Canada's fiduciary obligations. It has been noted that the current bill may pass in 2012 (personal communication, March 27, 2012). Table 8 summarizes the action theory for the legislation stage of AANDC's change theory.

\footnotetext{
${ }^{6}$ According to the AANDC interview respondent (personal communication, March 27, 2012)
} 
Table 8. AANDC Action Theory: Legislation

\begin{tabular}{ll}
\hline Action Theory Component & Required Inputs \\
\hline a) Resources & - None Specified \\
b) Implementing Organizations & - Parliament, Senate \\
c) Implementers & - Senators, Ministers, AANDC policy drafters \\
d) Associates/Partners & - First Nation, EC, HC, First Nations organizations \\
e) Ecological Context & - Bill S-8 may receive Royal Assent \\
f) Intervention Protocols & - The AANDC proposed legislative process, expert reports \\
g) Target Population & - First Nations communities
\end{tabular}

If legislation is passed, AANDC will work toward developing and implementing enforceable drinking water regulations including WTO minimum training levels and compliance with the aforementioned protocols. Terms related in the direction of regulation (4.3\% of factor references) appeared throughout the document review indicating that this instrument was highly regarded as a way forward. AANDC has stated that "developing regulations will take time and they will be implemented over a number of years in order to develop First Nation capacity and infrastructure" (AANDC, 2012b). Therefore, addressing the capital costs and training must commence prior to unveiling a regulatory regime. Table 9 summarizes this step's action theory.

Table 9: AANDC Action Theory: Regulation

\begin{tabular}{ll}
\hline Action Theory Component & Required Inputs \\
\hline a) Resources & - None specified \\
b) Implementing Organizations & - AANDC "in partnership" with First Nations \\
c) Implementers & - AANDC policy drafters \\
d) Associates/Partners & - First Nations, HC, EC, provinces, MTA municipalities, First \\
e) Ecological Context & - Currently no legally enforceable regulations for First Nations \\
f) Intervention Protocols & - Bill S-8, GCDWQ, informed by expert reports \\
g) Target Population & - First Nations communities
\end{tabular}

\footnotetext{
${ }^{7}$ Including the Report of the Expert Panel on Safe Drinking Water for First Nations and the Standing Senate Committee's final report on Safe Drinking Water for First Nations.
} 
Once an appropriate regulatory regime has been implemented, enforcement of regulations, standards, and protocols will take place. Terms pertaining to enforcement $(0.6 \%$ of factor references) and compliance $(1.9 \%)$ indicate that these factors were important issues that need addressing. Enforcement is meant as a mechanism to ensure that First Nations communities allocate resources to their water systems and train WTOs to comply with regulations. AANDC respondents indicated that there must be consequences for non-compliance because current trends for misdirecting transfer funds will continue. According to one interview respondent, "operators need transparency in funding transfers so that they can see that the department has allotted $\mathrm{X}$ to the First Nation" for given activities, such as O\&M, specific equipment, chemicals (personal communication, March 27, 2012). It was also suggested by the interview respondent that regulations will ensure operators are trained and provided resources as needed, particularly for addressing O\&M. Table 10 provides a summary of the enforcement intervention of AANDC's change theory.

\section{Table 10. AANDC Action Theory: Enforcement}

Action Theory Component Required Inputs
a) Resources
- None Specified

b) Implementing Organizations

- To be determined

c) Implementers

- To be determined

d) Associates/Partners

- First Nations, AANDC, HC, EC, First Nations groups

e) Ecological Context

- FNWWAP summative evaluation recommends housing an enforcement body outside of AANDC

f) Intervention Protocols

- None Specified

g) Target Population

- First Nations residents, WTOs, C\&Cs, CBWMs, EHOs

If regulations are to be enforced, monitoring practices must be bolstered.

Monitoring (4.1\% of factor references), sampling (4.2\%), and testing (4.2\%) practices 
were cited in the documents as critical to regulatory compliance. AANDC has articulated the importance of monitoring throughout the water system to ensure that protocols are adhered to and that the GCDWQ are not exceeded. These protocols take on a multibarrier approach to monitoring including four major aspects: water source, effective treatment, maintenance, and comprehensive testing (INAC, 2010b). Monitoring throughout the system will enable a future enforcement body to levy penalties for specific forms of non-compliance and allow First Nations to take appropriate corrective actions. A summary of the continuous monitoring action theory is shown in Table 11 .

Table 11. AANDC Action Theory: Continuous Monitoring

\begin{tabular}{ll}
\hline Action Theory Component & Required Inputs \\
\hline a) Resources & - $\$ 5 \mathrm{M}$ annually, unspecified amounts from FNWMS \& \\
& FNWWAP, totalling $\$ 1$ 16M between 2003-07 and \$54M \\
& annually between 2008-2013 \\
b) Implementing Organizations & - HC, First Nations \\
c) Implementers & - EHOs, WTOs, \& CBWMs \\
d) Associates/Partners & - Certified testing labs for corroboration, C\&Cs \\
e) Ecological Context & - It is difficult to test and train in remote communities \\
f) Intervention Protocols & - The Protocol, GCDWQ, \& other guides \\
g) Target Population & - First Nations community residents \\
\hline
\end{tabular}

It is important to note here that source water protection is an important and recognized factor in AANDC's change theory. Indeed, discussions pertaining to water source $(4.8 \%$ of factor references) and source protection $(2.4 \%)$ were consistently referenced within the documents reviewed. However, several notable aspects of the AANDC contamination risk assessment tool make it apparent that this element is not as high a priority as the others in the theory of change. First, within AANDC's definition of

\footnotetext{
${ }^{8}$ Including Testing Your Well Water: A Step-by-Step Checklist, A Guide for Environmental Health Officers and Community-Based Drinking Water Monitors, and Procedure Manual for Safe Drinking Water in First Nations Communities South of $60^{\circ}$
} 
risk, water source accounts for only $10 \%$ of the total risk score. Second, it is notable that all surface water, accounting for $29 \%$ (235) of all First Nations water systems, sources can be assessed for risk no lower than $5 \%$. Therefore, the management of such drinking water sources can only influence half of the risk score pertaining to surface water. As such, according to AANDC's change theory human intervention for surface water sources can only prevent surface water from being high-risk, not medium- or low-risk. Thirdly, AANDC indicates in its definition of contamination risk that this aspect of risk does not overlap with the other elements of the definition of risk, whereas O\&M, reporting, and infrastructure investments are intimately tied to WTO qualifications and training (AANDC, 2011d). Therefore, AANDC implies that protecting surface water will have less impact upon drinking water quality at consumption than will WTO training. Finally, source water protection is the only component of the AANDC's definition of contamination risk that is not at least partially funded by the federal government. In sum, although AANDC promotes source water protection as part of the change theory, it is low priority relative to the other elements of the change theory. Table 12 summarizes the action theory for AANDC's final step.

Table 12. AANDC Action Theory: Source Water Protection

\begin{tabular}{ll}
\hline Action Theory Component & Required Inputs \\
\hline a) Resources & - First Nations allocations \\
b) Implementing Organizations & - First Nations \\
c) Implementers & - C\&Cs \\
d) Associates/Partners & - EC, watershed authority, provincial governments, nearby \\
e) Ecological Context & - Funicipalities \\
f) Intervention Protocols & - None specified \\
g) Target Population & - First Nations communities \\
\hline
\end{tabular}




\section{Environment Canada}

A total of 31 documents from EC were reviewed and a content analysis used to determine the department's stated problem definition and theory for change regarding First Nations drinking water quality. These documents addressed a wide range of topics from water quality governance and legislation to measures taken for protecting watersheds, such as the great lakes. EC's approach for addressing issues of drinking water quality in First Nations communities focuses on protecting the source water from becoming contaminated. Contamination prevention ensures that raw water is easier to treat for drinking. Such prevention will also mitigate against significant health issues should human error occur within the drinking water treatment processes. Although this theme is within the purview of EC's role according to the analysis of the Expert Panel on First Nations Drinking Water (Swain, Loutit, \& Hrudey, 2006), the department views its responsibilities as limited. Indeed, an interview respondent from EC suggests that such responsibilities lie with the overseeing department/government, such that AANDC is responsible for source water protection in First Nations and provincial governments are responsible elsewhere (personal communication, April, 26, 2012). As a result, source water protection has been left neglected. A notable element of EC's change theory is its limited focus on O\&M, which reflects the idea that if source water is protected sufficiently quality drinking water will require fewer treatment efforts. A second notable element is the placement of drinking water regulation at the end of the change theory, indicating that all other elements must first be addressed prior to enforcing standards. The assumption here is that First Nations be unable to comply with such standards immediately. 
Problem definition. A variety of issues related to source water contribute to the primary issue of drinking water quality. Issues of contamination (3.8\% of EC factor references) are raised throughout the documents reviewed including chemical (3.9\%) contaminants and microbiological $(0.8 \%)$ contaminants. The emphasis on chemical contamination indicates the magnitude of the impact. For example, a single "drop of oil can render up to 25 litres of water unfit for drinking," and one gram of "2-4-D (a common household herbicide) can contaminate ten million litres of drinking water" (EC, 1992). According to the interview respondent, "the quantity of contamination doesn't really matter, instead it is the nature of the substance" (personal communication, April 26,2012 ). Furthermore, pollution (3.6\% of factor references) was commonly cited, regarding agriculture, industry, and individual homes. EC's problem definition pertaining to drinking water quality includes contamination prevention programs. Water sources such as groundwater ( $12.5 \%$ of factor references) and surface water (1.6\%) including rivers $(16.9 \%)$ and lakes $(12.6 \%)$, were discussed with high significance. Further, source water protection ( $5.2 \%$ of factor references) was the most referenced activity for ameliorating drinking water quality issues. The respondent articulated that "part of the problem is industry contamination; however, the issue goes deeper than that" to include a general disrespect of water, which results in a variety of contaminants (personal communication, April, 26, 2012). EC suggests that "preventing contamination in the first place is by far the most practical solution" (EC, 2012a) as noted:

Good quality drinking water is free from disease-causing organisms, harmful chemical substances, and radioactive matter; it tastes good, is aesthetically appealing, and is free from objectionable colour or odour (EC, 2006a).

Theory of change. EC facilitates the protection the environment, conservation of natural heritage, and protection of Canadians through awareness of environmental 
issues (EC, 2012b). The department's mandate is to "preserve and enhance the quality of the natural environment; $[. .$.$] conserve Canada's renewable resources; conserve and$ protect Canada's water resources, $[. .$.$] and coordinate environmental policies and$ programs for the federal government" (EC, 2012b). These responsibilities centre on environmental protection. With regard to drinking water, EC's change theory is that with chemical treatment, water will be safe for human consumption, and with increased awareness of water safety, only regular maintenance will be necessary over the longer term. As such, health risks due to drinking water quality issues due to human error or negligence will be minimized. The department suggests that its research can be "used to set Canada-wide standards" for environmental protection (EC, 2012b).

With regard to First Nations, EC states that it "works with the governments, communities, and organizations of the Aboriginal Peoples of Canada to advance the department's programs and priorities and to support Aboriginal environmental stewardship and objectives" (EC, 2012b). The department explains that its role is to support First Nations stewardship, including that of drinking water sources. This is consistent with the analysis within the Report of the Expert Panel on First Nations Drinking Water, which indicates that EC is responsible for providing advice and guidance to First Nations in developing source water protection and sustainable water usage planning (Swain, Loutit, \& Hrudey, 2006).

EC's theory of change is dependent on important activities including: contributing to First Nations drinking water quality through reducing contamination; and regulating wastewater as a potential threat to source water. 
With respect to these activities, the documents reviewed do not directly address drinking water quality of First Nations communities, as EC's role is limited. Managing water is a shared responsibility necessitating "close cooperation and collaboration among all levels of government, Aboriginal peoples, and the public" (EC, 2006b). As such, First Nations should have a prominent role in their own drinking water governance (EC interview respondent, personal communication, April 26, 2012). There is a need to define the roles of the drinking water governance actors to ensure a comprehensive change theory is implemented. In this respect, the interview respondent noted that "colleagues at $\mathrm{HC}$ and [AANDC] are not informed of EC's role or the limitations of [its] role" (personal communication, April 26, 2012), and as a result source water protection has often come as an afterthought, despite the alleged use of a multi-barrier approach to drinking water quality. Most important in this regard, wastewater management is critical to protecting drinking water sources from becoming contaminated. The respondent noted that "by dealing with wastewater [EC is] indirectly contributing to drinking source water [because] wastewater is the largest pollutant to surface water in terms of volume" (personal communication, April 26, 2012).

Change model. EC's comprises seven sequential steps shown in Figure 3. Solid arrows identify direct pathways in the change theory and dotted arrows illustrate feedback loops. 
Figure 3. EC's Change Model

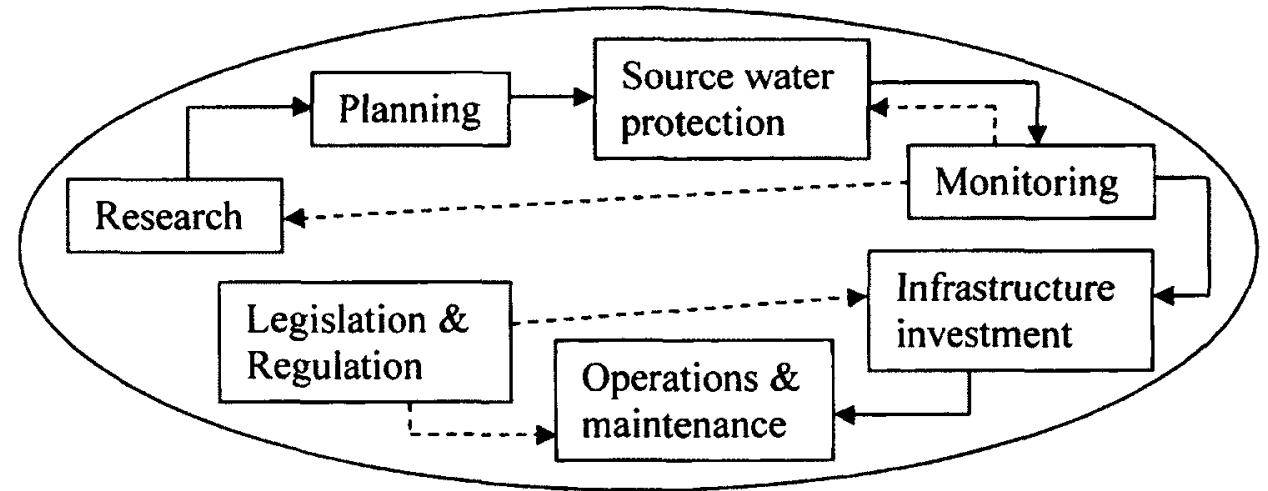

The first step in EC's change theory emphasizes researching and assessing source water and human impact on source water. Such research (3.1\% of factor references) includes determining how source water interacts with the ecosystem, an analysis of contaminants that pervade the water system, and environmental impact assessments of industry and business development. ${ }^{9}$ The EC interview respondent indicated that EC's role primarily regards "water surveying and meteorology studies to measure the quantity and quality of water supplies" (personal communication, April 26, 2012). Habitat research is of the upmost importance because what is bad for fish can only be detrimental to drinking water. ${ }^{10}$ Several research activities have begun including the Global Environmental

\footnotetext{
${ }^{9}$ Environmental assessments (1.7\% of factor references) were emphasized but are likely to be reduced in scope, as the Omnibus Bill C.38 will likely be passed in 2012. This bill seeks to move "toward a "one project, one review' system for reviews of major projects by recognizing provincial processes as substitutes or equivalents to federal ones as long as they meet the requirements under the Canadian Environmental Assessment Act" (GOC, 2012). Further, the Bill seeks to amend the Canadian Environmental Assessment Act such that the Canadian Environmental Assessment Agency is required to make quicker decisions on required assessments ( 45 days) and shorten the maximum assessment process (to 24 months). The interviewee indicated that these changes are worrisome and could contribute to propagating the problems with drinking water quality in Canada.

${ }^{10}$ It is significant to note that the EC official stressed the importance of the Fisheries Act throughout the interview. Specifically, he detailed the anticipated changes to the act through the Omnibus Bill C-38, indicating that immediately following the passing of the Bill, the federal government will be allowed to make exceptions to the strict regulations contained within the Fisheries Act. His concerns were that eventually provisions within the Fisheries Act protect waters that contain fish used for commercial,
} 
Monitoring System (GEMS)/Water program, which provides "evidence-based information on the state and trends of global inland water quality" by assessing water quality of a variety of sources throughout Canada. Other areas of research conducted include environmental prediction, biological testing (of fish and aquatic animals health), emergency responses, risk assessments (contamination, global warming effects on water), and the use of technology for water quality. Table 13 summarizes the action theory of research within EC's change theory.

Table 13. EC Action Theory: Research

\begin{tabular}{ll}
\hline Action Theory Component & Required Inputs \\
\hline a) Resources & $\begin{array}{r}\text { - } \begin{array}{l}\text { } 2.5 \mathrm{M} \text { for GEMS over five years since 2009, \$3.75M funded } \\
\text { to Lethbridge University and the University of Saskatchewan } \\
\text { for water quality studies, other unspecified research } \\
\text { spending }\end{array} \\
\end{array}$ \\
$\begin{array}{ll}\text { b) Implementing Organizations } \\
\text { c) Implementers }\end{array}$ & - EC, provinces, research institutions, universities \\
d) Associates/Partners & - First Nations, United Nations, municipalities, USA \\
e) Ecological Context & - None specified, but scientific method likely \\
f) Intervention Protocols & - None specified, but scientific method likely \\
g) Target Population & - Individuals and ecosystems reliant on specific water systems \\
\hline
\end{tabular}

The purpose of the research is to build a knowledge base and subsequently develop a plan for source water protection by utilizing knowledge obtained from research. There was emphasis on planning ( $2.3 \%$ of factor references), as EC's role is to provide advice to First Nations communities regarding source water protection. The planning process described the protection of entire watersheds. Further, the EC interview respondent indicated that provinces, municipalities, First Nations, and the federal government should all work together to protect watersheds, as opposed to individual 
water systems, but noted that such protection has yet to occur because "the department will only take care of EC-owned water systems" (personal communication, April 26, 2012). As a consequence, provincial watershed protection committees do not allocate enough seats to engage all First Nations communities. For example, there may be ten First Nations communities within a watershed, but only two seats on the committee are offered for First Nations people, and "as a result, there is not a lot of participation" (personal communication, April 26, 2012). Table 14 summarizes the EC's action theory for planning.

Table 14. EC Action Theory: Planning

\begin{tabular}{ll}
\hline Action Theory Component & Required Inputs \\
\hline a) Resources & - Unspecified amount \\
b) Implementing Organizations & - AANDC, HC, First Nations \\
c) Implementers & - Departmental policy drafters, C\&Cs \\
d) Associates/Partners & - Research institutions, Universities, WTOs, EC \\
e) Ecological Context & - Source protection planning to-date has been limited \\
f) Intervention Protocols & - None specified \\
g) Target Population & - First Nations community residents
\end{tabular}

It follows that once plans are developed, implementing source water protection must occur. As noted, source water (44.3\% of factor references) and protection (5.2\%) were the most referenced aspects of drinking water. In implementing these plans, clear guidelines (2.4\% of factor references) are required. Such guidelines must be "scientifically determined and indicate the maximum allowable concentration of substances for a particular water use", whereas objectives "specify the concentrations of substances permissible for all intended water uses at a specific location" (EC, 1992). EC articulates that a lack of standards, objectives, and guidelines lead to a variety of health problems, and that much effort has been expended on developing tools to support source 
water protection, but that these tools remain under-utilized because EC does not distribute them directly to First Nations. Instead, AANDC is responsible for disseminating these tools. The action theory for source water protection according to EC is summarized in Table 15.

\section{Table 15. EC Action Theory: Source Water Protection}

\begin{tabular}{ll}
\hline Action Theory Component & Required Inputs \\
\hline a) Resources & - None specified \\
b) Implementing Organizations & - EC, First Nations, municipalities, provinces \\
c) Implementers & - All residents (guided by implementing organizations) \\
d) Associates/Partners & - Watershed authorities, farmers, private sector \\
e) Ecological Context & - Current source water protection is jurisdictional \\
f) Intervention Protocols & - Plans developed in previous component \\
g) Target Population & - All Canadian residents \\
\hline
\end{tabular}

If plans are implemented using guidelines, it follows that source water can be monitored to ensure that the plans are implemented correctly. Monitoring ( $3.5 \%$ of factor references) is cited throughout the documents. Water quality monitoring is inclusive of "a scientifically designed system of long-term, standardized measurement, systematic observation, evaluation and reporting of water quality in order to define status and/or trends" (EC, 2011a). There are two important ideas: first, water quality issues can be identified and dealt with as they arise; and second, data obtained from water monitoring can inform source water quality research. Table 16 summarizes EC's action theory for monitoring. Resource estimates are taken from AANDC. 
Table 16. EC Action Theory: Monitoring

\begin{tabular}{ll}
\hline Action Theory Component & Required Inputs \\
\hline a) Resources & - $\$ 5$ annually, unspecified amounts from FNWMS \& \\
& FNWWAP, totalling $\$ 116 \mathrm{M}$ between 2003-07 and \$54M \\
& annually between 2008-2013 for HC-focused monitoring, \\
& additional resources not specified for EC-focused monitoring \\
b) Implementing Organizations & - EC, HC, First Nations, provinces, municipalities \\
c) Implementers & - EHOs, WTOs, CBWMs, researchers \\
d) Associates/Partners & - Research institutions, universities, UN, USA, all residents \\
e) Ecological Context & - EC-focused monitoring primarily regards raw water \\
f) Intervention Protocols & - None specified \\
g) Target Population & - All Canadian residents
\end{tabular}

If source water protection plans are implemented and monitoring activities are taking place, it follows that infrastructure and resources must be available to effectively treat raw water for safe drinking. Capital ( $0.9 \%$ of factor references) and infrastructure investments $(1.7 \%)$ were noted as needs for treating water, including for example that "large-scale investments are addressing the need for improved water and wastewater treatment facilities to meet the health and environmental needs of First Nations and Inuit communities" (EC, 2012c). Indeed, the EC interview respondent noted that several First Nations communities have aging and outdated infrastructure (personal communication, April 26, 2012). Table 17 provides a summary of EC's action theory for infrastructure investment. Resource estimates are again taken from AANDC. 
Table 17. EC Action Theory: Infrastructure Investment

\begin{tabular}{|c|c|}
\hline Action Theory Component & Required Inputs \\
\hline a) Resources & $\begin{array}{l}\text { - } \$ 820 \mathrm{M} \text { from investments made between } 2006-2010 \text {, } \\
\text { unspecified amount from } \$ 330 \mathrm{M} \text { biannual A-Base } \\
\text { allocations from } 2008-13, \$ 165 \mathrm{M} \text { from Canada's Economic } \\
\text { Action Plan }\end{array}$ \\
\hline b) Implementing Organizations & - First Nations, AANDC, contractors \\
\hline c) Implementers & - C\&Cs, departmental policy drafters, contractors \\
\hline d) Associates/Partners & - $\mathrm{HC}, \mathrm{PWGSC}, \mathrm{CMHC}$ \\
\hline e) Ecological Context & - Ongoing investment is needed \\
\hline f) Intervention Protocols & - The Protocol \\
\hline g) Target Population & - First Nations community residents \\
\hline
\end{tabular}

If infrastructure needs are addressed, then ongoing regular O\&M can be implemented. Terms pertaining to both operation ( $1.4 \%$ of factor references) and maintenance (1.1\%) occurred throughout the documents, but in low frequency. The EC interview respondent indicated that there are inadequate resources allocated for O\&M activities (personal communication, April 26, 2012). There is a challenge with retaining WTOs because funds are not available to both pay them competitively. The interview respondent prescribed promoting the value of WTOs on the same level as health service providers, Chiefs and Councillors, and other high ranking band administrators. Table 18 summarizes the O\&M action theory according to EC. Resources estimates within this table are taken from AANDC. 
Table 18. EC Action Theory: Operations and Maintenance

\begin{tabular}{ll}
\hline Action Theory Component & Required Inputs \\
\hline a) Resources & $\begin{array}{l}\text { - } 80 \% \text { funded by AANDC, 20\% by FNs; \$431M between } \\
2006-10, \text { unspecified amount from \$330M from AANDC's } \\
\text { biannual A-Base allocations from 2008-13, \$40M in } \\
\text { additional departmental allocations per year }\end{array}$ \\
$\begin{array}{ll}\text { b) Implementing Organizations } & \text { - AANDC, First Nations } \\
\text { c) Implementers } & \text { - WTOs } \\
\text { d) Associates/Partners } & \text { - HC, circuit rider trainers, C\&Cs } \\
\text { e) Ecological Context } & \text { - WTOs not valued for their work; funding can be insufficient } \\
\text { f) Intervention Protocols } & \text { - The Protocol } \\
\text { g) Target Population } & \text { - First Nations community residents }\end{array}$
\end{tabular}

EC suggests that once O\&M activities are funded sufficiently and, legislation and enforceable regulations can be enacted. Although legislation ( $0.6 \%$ of factor references) and regulation (1.3\%) were not referenced significantly, their inclusion in the change theory is explicit. EC states that "elements of a successful drinking water program can include state-of-the art facilities, operation certification, an effective compliance assurance program with emergency response protocols, and measures to ensure public confidence" (EC, 2003 emphasis added). The respondent indicated that it may be to First Nations' benefit that regulations come into effect before resources are made available. That is, First Nations could seek additional funding from AANDC by stating "help us not break the law" (personal communication, April 26, 2012). However, if occurred, it is likely that hundreds of communities would adopt this approach and issues of inadequate resources would remain prevalent due to the current level of financial resources available. The action theory for the legislation and regulation step of the change theory, according to EC, is summarized in Table 19. 
Table 19. EC Action Theory: Legislation and Regulation

\begin{tabular}{ll}
\hline Action Theory Component & Required Inputs \\
\hline a) Resources & - None specified \\
b) Implementing Organizations & - AANDC, HC, First Nations \\
c) Implementers & - Departmental policy drafters, C\&Cs \\
d) Associates/Partners & - EC, provinces, municipalities, First Nations groups \\
e) Ecological Context & - Infrastructure and operations and maintenance issues must \\
f) Intervention Protocols & - None specified \\
g) Target Population & - First Nations community residents
\end{tabular}

\section{Health Canada}

A total of 16 documents focusing on drinking water quality of First Nations communities were reviewed from Health Canada's First Nations and Inuit Health Branch to develop the problem definition and theory of change. The majority of the documents were drafted as technical advice or guidelines for First Nations, including several web pages that describe how and when to issue water advisories/orders when a water emergency occurs. Other documents were documents providing a procedure for Community-Based Drinking Water Monitors (CBWMs) and HC employed Environmental Health Officers (EHOs) who monitor the quality of drinking water on reserves. Operational reports detailing current trends for water treatment in First Nations communities were also reviewed. The results of the document review were supplemented by interviews with two senior officials from HC who work specifically on First Nations communities' water quality.

HC's approach to addressing drinking water quality issues of First Nations communities focuses on the treatment of water throughout the water system. Although HC greatly recognizes the need for improving First Nations compliance regarding 
drinking water quality monitoring, the department's change theory emphasizes treatment and the capacity to fulfill treatment requirements. Notable elements of the $\mathrm{HC}$ change theory include the notion that capacity for management should first be built up before regulations are developed and enforced, as well as the apparent need for First Nations community engagement.

Problem definition. The most common issues pertaining to drinking water of First Nations communities result from inconsistent and inadequate treatment $(7.3 \%$ of $\mathrm{HC}$ factor references) and disinfection (3.7\%) processes. When treatment processes fail, the water system fails and action must be taken to remedy issues related to treatment failure. " The most common reasons for issuing drinking water advisories (DWAs) in First Nations communities are due to contamination related to "inadequate disinfection or disinfectant residuals (34\%); [and] equipment malfunction during treatment or distribution (28\%)" (HC, 2009) as noted:

Many First Nations communities face the same challenges in providing safe drinking water as do other small, remote or isolated communities, such as difficulties in finding and retaining qualified water treatment plant operators [...], community decisions to lower or turn off the chlorinator because of community concerns about the taste of chlorine in drinking water after treatment, [and] the time required to perform upgrades or replacements to a water facility in a community $(\mathrm{HC}, 2011 \mathrm{a})$.

$\mathrm{HC}$ respondents suggest that a variety of factors prevent First Nations communities from realizing sound water treatment practices including community size and location. These factors contribute to a lack of access to adequate training and infrastructure resources related to treatment. In addition, high-tech facilities are often built in First Nations communities without adequate capacity to operate and maintain them. $\mathrm{HC}$ also noted the regulations gap between First Nations and non-First Nations communities, such that

"These documents assume that drinking water contamination ( $2.7 \%$ of factor references) is inevitable. HC indicates that contamination primarily concerns microbiological ( $5.8 \%$ of factor references). 
standardized water treatment processes cannot be enforced in First Nations communities. $\mathrm{HC}$ interview respondents suggested that many First Nations communities lack understanding of the importance of O\&M processes and costs as a result (personal communication, April 3, 2012).

Theory of change. HC's mandate is to improve "the lives of all of Canada's people and to making this country's population among the healthiest in the world as measured by longevity, lifestyle and effective use of the public health care system" (HC, 2012a). HC's objective most pertinent to drinking water quality is to "prevent and reduce risks to individual health and the overall environment" (HC, 2012a). HC's theory of change is to prevent and mitigate against health-related drinking water contamination risks. According to the analysis by the Expert Panel on Drinking Water for First Nations, $\mathrm{HC}$ is responsible for oversight on mandates pertaining to safe drinking water monitoring at tap, CBWM training, and developing guidelines for drinking water quality and best practices (Swain, Loutit, \& Hrudey, 2006).

The First Nations and Inuit Health Branch (FNIHB) is responsible for "helping people stay healthy, and prevent chronic and contagious diseases" ( $\mathrm{HC}, 2012 \mathrm{~b})$. The strategic outcome of this branch is for First Nations and Inuit communities to "receive health services and benefits that are responsive to their needs so as to improve their health status" (HC, 2012a). The branch addresses First Nations communities' drinking water quality by monitoring to prevent and mitigate against health related contaminants.

HC's theory for changing First Nations drinking water quality issues is built on a foundation of several activities including: a multi-barrier approach to drinking water protection; engaging with First Nations, and raising First Nations peoples' awareness of 
drinking water quality issues. First, a multi-barrier approach consists of three elements: source protection, treatment, and system protection (HC, 2010b). HC focuses on the last two elements. Second, although documents refer to engagement $(0.2 \%$ of factor references) infrequently, notions of involvement $(0.9 \%)$ and consultation $(0.8 \%)$ are recognizable. HC interview respondents noted that the key toward engagement is building mutual trust, which will take a significant amount of time as it will need to occur "one community at a time" through two-way communication, knowledge recognition, and knowledge sharing (personal communication, April 3, 2012). Finally, notions of education $(0.5 \%$ of factor references), understanding $(0.5 \%)$, and awareness $(0.9 \%)$ were also identified. Awareness and education were considered vital given that an $\mathrm{HC}$ study found that "just over $75 \%$ of First Nations respondents indicated that information on water quality testing procedures, testing frequency, acceptable levels of contaminants in tap water and tap water quality would help them feel more confident toward the quality of their drinking water" (HC, 2010b). Further, the interview respondents suggested that many First Nations band councils must understand the importance of treatment and monitoring practices in order to effectively allocate necessary funds to these activities (personal communication, April 3, 2012). 
Change model. HC's change model comprises seven sequential steps (see Figure 4). Note that solid arrows identify direct pathways and dotted arrows illustrate feedback loops.

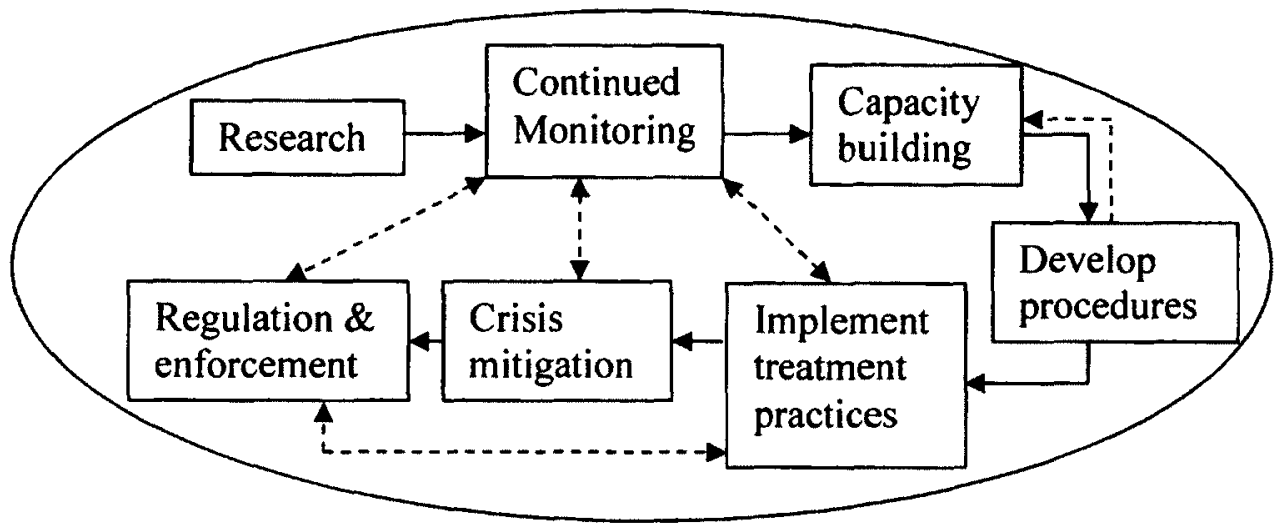

First, research is critical for addressing the problem that First Nations drinking water is not sufficiently treated for consumption. The documents revealed a need to better understand the contexts surrounding water disease events (WDEs) in order to prevent and address similar future outbreaks. Although the term "research" only accounts for only $0.5 \%$ of HC's factor references identified, terms relating to assessments (2.0\%) and evaluations (2.0\%) are relatively frequent. The two latter terms refer to determining threats to water systems in their relation to potential WDEs. It is also the case that "there is no national surveillance system specifically for waterborne disease outbreaks" (HC, $2011 \mathrm{~b})$. The design of policies to prevent drinking water contamination outbreaks is stagnated due to the lack of information regarding the characteristics and causes of such events. Therefore, data gathering of WDE threats is critical to anticipate and address such events. Table 20 summarizes the action theory for the research step according HC. 
Table 20. HC Action Theory: Research

\begin{tabular}{|c|c|}
\hline Action Theory Component & Required Inputs \\
\hline a) Resources & $\begin{array}{l}\text { - An unspecified amount from FNWMS \& FNWWAP, } \\
\text { totalling } \$ 115.9 \mathrm{M} \text { from 2003-07 and } \$ 54 \mathrm{M} \text { bi-annually from } \\
2008-13\end{array}$ \\
\hline b) Implementing Organizations & - HC, First Nations, regional health units, municipalities \\
\hline c) Implementers & $\begin{array}{l}\text { - Researchers, epidemiologists, Communicable Disease Nurse } \\
\text { Coordinators, Regional Medical Officers, EHOs }\end{array}$ \\
\hline d) Associates/Partners & - C\&Cs, universities, provinces \\
\hline e) Ecological Context & - Population size, remoteness, and water source are key foci \\
\hline f) Intervention Protocols & - Procedural guidelines ${ }^{12}$ \\
\hline g) Target Population & - All Canadian residents \\
\hline
\end{tabular}

It follows that if there is an adequate understanding of WDE threats First Nations communities can then monitor drinking water to identify problems as they arise. Monitoring (7.9\% of factor references) drinking water by regular sampling (10.6\%) and testing (3.7\%) was observed as critical in the documents reviewed. HC interview respondents indicated that monitoring was the most important factor in addressing drinking water issues and suggested that Water Safety Plans (WSPs) would be a significant step forward in resolving water system threats. They described managing WSPs through computerized systems as ideal in managing comprehensive risk assessment and risk management information regarding water systems. This information would then be used to assess threats to public health related to drinking water from source to tap. They also stated that WSPs "will bring together awareness of issues pertaining to source water protection, treatment, and what is needed for appropriate operations and maintenance" and that they will highlight "things need to be dealt with immediately; as soon as possible" (personal communication, April 3, 2012). Although this step is a crucial component in the change theory, $\mathrm{HC}$ documents indicate that "water

\footnotetext{
${ }_{12}$ Procedural Guidelines for Waterborne Disease Events in First Nations Communities South of $60^{\circ}$
} 
quality monitoring within First Nations communities is not sufficient to protect public health", as this practice provides only information rather than providing the capacity and resources to effectively address issues that are identified (HC, 2010b). The action theory for continued monitoring according to $\mathrm{HC}$ is summarized in Table 21.

Table 21. HC Action Theory: Continued Monitoring

\begin{tabular}{ll}
\hline Action Theory Component & Required Inputs \\
\hline a) Resources & - $\$ 5$ annually, unspecified amounts from FNWMS \& \\
& FNWWAP, totalling $\$ 116 \mathrm{M}$ between 2003-07 and \$54M \\
& annually between 2008-2013 \\
b) Implementing Organizations & - HC, First Nations \\
c) Implementers & - EHOs, WTOs, CBWMs \\
d) Associates/Partners & - Certified testing labs for corroboration, AANDC, C\&Cs \\
e) Ecological Context & - It is difficult to test and train in remote communities \\
f) Intervention Protocols & - The Protocol, GCDWQ, guidelines \\
g) Target Population & - First Nations community residents \\
\hline
\end{tabular}

Next, if drinking water systems are monitored then First Nations will require the capacity ( $0.6 \%$ of factor references) and resources $(0.4 \%)$ to treat unsafe water and remedy quality issues. HC noted a high turnover rate among EHOs, CBWMs, and WTOs, which diminishes the expertise and capacity for continuous and consistent monitoring and treatment. However, $\mathrm{HC}$ officials indicated that increasing First Nation communities' capacity to operate and maintain treatment systems through training $(1.6 \%$ of factor references) and retention (0.1\%) are essential pieces of the change theory. $\mathrm{HC}$ interview respondents suggested that nearby non-First Nations communities tend to "poach" trained

\footnotetext{
${ }^{13}$ Additional costs are recorded for EHO and CBWMs. Average salaries are \$72, 945 for the $96 \mathrm{EHOS}$ (totalling \$7M) and \$6,000 for the 592 CBMWs (\$3.6M)

${ }^{14}$ Including Testing Your Well Water: A Step-by-Step Checklist, A Guide for Environmental Health Officers and Community-Based Drinking Water Monitors, and Procedure Manual for Safe Drinking Water in First Nations Communities South of $60^{\circ}$
} 
WTOs by offering greater incentives than First Nations communities can afford (personal communication, April 3, 2012).

In addition, a highly technically advanced infrastructure is sometimes introduced into communities without human or financial resources to operate and maintain it; "if people are knowledgeable then they may be able to use current systems with only minor treatment issues" (personal communication, April 3, 2012). That is, the capacity of First Nations communities must be addressed to resolve issues identified by consistent monitoring and adequate training. Table 22 summarizes the capacity building action theory according to $\mathrm{HC}$.

Table 22. HC Action Theory: Capacity Building

\begin{tabular}{ll}
\hline Action Theory Component & Required Inputs \\
\hline a) Resources & - Unspecified amount (provided by AANDC) \\
b) Implementing Organizations & - AANDC, HC, First Nations \\
c) Implementers & - First Nations, WTOs, Circuit rider trainers, contractors \\
d) Associates/Partners & - CMHC, PWGSC \\
e) Ecological Context & - Community location and housing impact funds required \\
f) Intervention Protocols & - The Protocol \\
g) Target Population & - First Nations community residents, C\&Cs \\
\hline
\end{tabular}

Next, there is a need for developing procedural guidelines and manuals to support training and monitoring including training instruments and tools which CBMWs, EHOs, and WTOs can utilize. HC states in the preamble to these documents that they have been developed to increase "its own [HC's] capacity and the capacity of First Nations communities to sample and test drinking water quality" (HC, 2011a). Funding was tied to developing these documents under a mandate to begin "Building First Nations Capacity" 
(HC, $2011 \mathrm{a})$. Terms relating to manuals $(5.9 \%$ of factor references), procedures $(8.9 \%)$, protocols (1.2\%), and guidelines $(3.9 \%)$ were significant. ${ }^{15}$

Interviews with $\mathrm{HC}$ officials indicated that there are sometimes no O\&M manuals for highly complex water systems. They articulated that issues identified through WSPS could be addressed using best practices outlined in operating manuals. However, the documents suggest that some flexibility within these tools must be maintained in order to account for different water sources, locations, and population sizes. That said, several barriers to following the procedures were identified: "retention of trained personnel to monitor drinking water quality, community access to resources needed for analyzing samples, standardization of procedures, and implementation of work plans at the regional level" (HC, 2010b). Table 23 provides a summary of the action theory for the developing procedures according to $\mathrm{HC}$.

Table 23. HC Action Theory: Develop Procedures

\begin{tabular}{ll}
\hline Action Theory Component & Required Inputs \\
\hline a) Resources & - An unspecified amount from FNWMS \& FNWWAP, \\
& totalling \$115.9M from 2003-07 and \$54M bi-annually from \\
& $2008-13$ \\
b) Implementing Organizations & - HC, EC, AANDC \\
c) Implementers & - Departmental policy drafters \\
d) Associates/Partners & - Provinces, First Nations groups, C\&Cs \\
e) Ecological Context & - Treaty rights are important to recognize during development \\
f) Intervention Protocols & - None specified \\
g) Target Population & - CBWMs, EHOs, WTOs, C\&C
\end{tabular}

It then follows that if capacity issues are ameliorated and staffs have the tools and knowledge necessary to implement all monitoring and treatment processes articulated in

\footnotetext{
${ }^{15}$ Including the GCDWQ, the Procedural Guidelines for Waterborne Disease Events in First Nations Communities South of $60^{\circ}$, toolkits for testing individual wells, How to Protect the Water in Your Pipes, a procedure manual for addressing DWAs, a guide for environmental protection guidelines, and procedural manual for ensuring safe drinking water, and AANDC protocols for drinking water systems.
} 
procedural documents, these activities will lead to effective treatment of drinking water in First Nations communities. Drinking water treatment (7.3\% of factor references) was common throughout the documents, whereby "inadequate disinfection or disinfectant residuals was the most commonly cited reason in [the issuance of] $56 \%$ of' DWAs in First Nations communities from 1995-2007 (HC, 2009). Therefore, following best practice procedures for ensuring effective water treatment is a key component of the HC change process. HC's action theory for implementing drinking water treatment is summarized in Table 24. Resource estimates are provided by AANDC.

Table 24. HC Action Theory: Implement Treatment

\begin{tabular}{ll}
\hline Action Theory Component & Required Inputs \\
\hline a) Resources & $\begin{array}{l}\text { - } 80 \% \text { funded by AANDC, 20\% by First Nations; } \$ 431 \mathrm{M} \\
\text { between } 2006-10, \text { unspecified amount from } \$ 330 \mathrm{M} \text { from } \\
\text { biannual A-Base allocations from 2008-13, } \$ 40 \mathrm{M} \text { in } \\
\text { additional departmental allocations per year }\end{array}$ \\
$\begin{array}{ll}\text { b) Implementing Organizations } & \text { - First Nations } \\
\text { c) Implementers } & \text { - WTOs } \\
\text { d) Associates/Partners } & \text { - C\&Cs, MTA municipalities, contracted organizations } \\
\text { e) Ecological Context } & \text { - There is no enforcement regime to ensure use of funds } \\
\text { f) Intervention Protocols } & \text { - The Protocol, GCDWQ, provincial legislation } \\
\text { g) Target Population } & \text { - First Nations community residents }\end{array}$
\end{tabular}

$\mathrm{Next}, \mathrm{HC}$ recognizes that drinking water contamination is likely to occur regardless whether procedures are followed or not. Weather, water source degradation, and infrastructure, and capital malfunctions and deterioration are common and beyond the scope of WTO control (HC, 2009). As such, several procedural documents (six) were designed to manage a crisis when contamination $(2.7 \%$ of factor references) occurs. A need for emergency response planning was articulated frequently in the documents $(2.1 \%$ of factor references), meaning that processes to mitigate against harm were considered. Continuous monitoring of all processes in a water system contributes to reducing such 
harm provided that they are addressed in due time. Table 25 summarizes HC's action theory for crisis mitigation.

Table 25. HC Action Theory: Crisis Mitigation

\begin{tabular}{ll}
\hline Action Theory Component & Required Inputs \\
\hline a) Resources & $\begin{array}{l}\text { - As needed, First Nations allocate funds to develop } \\
\text { emergency response manuals }\end{array}$ \\
\begin{tabular}{ll} 
b) Implementing Organizations & - First Nations, HC \\
c) Implementers & - C\&Cs, WTOs \\
d) Associates/Partners & - AANDC, provinces, regional health units \\
e) Ecological Context & - HC provides advice to First Nations \\
f) Intervention Protocols & - Procedural manuals \\
g) Target Population & - First Nations community residents \\
\hline
\end{tabular}
\end{tabular}

Next, if each of the previous steps has occurred, HC suggests that a regulatory framework could be implemented as a final step. Such a framework can provide a legal mechanism for enforcing compliance including infrastructure design. Although the terms regulation $(0.7 \%$ of factor references $)$ and legislative frameworks $(0.7 \%)$ were cited infrequently, $\mathrm{HC}$ interview respondents stated that "the smart implementation of legislation" is necessary, but that regulations are only one element of the solution and that capacity issues must first be addressed (personal communication, April 3, 2012). HC's action theory for regulation and enforcement is provided in Table 26.

\footnotetext{
${ }^{16}$ Procedural Guidelines for Waterborne Disease Events in First Nations Communities South of $60^{\circ}$ and Procedure for Addressing Drinking Water Advisories in First Nations Communities South of $60^{\circ}$
} 
Table 26. HC Action Theory: Regulation and Enforcement

\begin{tabular}{ll}
\hline Action Theory Component & Required Inputs \\
\hline a) Resources & - None specified \\
b) Implementing Organizations & - AANDC, HC, EC, First Nations \\
c) Implementers & - Departmental policy drafters, C\&Cs \\
d) Associates/Partners & - First Nations groups \\
e) Ecological Context & - First Nations treat rights are important to recognize \\
f) Intervention Protocols & - None specified \\
g) Target Population & - First Nations community residents \\
\hline
\end{tabular}

\section{Assembly of First Nations and Chiefs of Ontario}

Two organizations were selected as proxies to represent First Nations regarding the problem definition and theory of change. In total, 31 documents were analyzed: $51.6 \%$ (16) from the AFN and $48.4 \%$ (15) from the $\mathrm{CoO}$. Much of the published information on the AFN and $\mathrm{CoO}$ web pages related to Bills S-11 and S-8, which are proposed federal legislation to enable the establishment of regulations for drinking water systems on reserves. Six of the documents were letters from the AFN, and three were letters from the $\mathrm{CoO}$, to those individuals involved in the drafting of or provisions regarding the proposed legislation, including the Minister of AANDC, Dr. Harry Swain (lead expert on the Expert Panel on First Nations Drinking Water), Senator Denis St. Germain (chair of the Standing Senate Committee on Aboriginal Peoples), and First Nations community organizations and governments. Little other information was available regarding First Nations community drinking water quality. Data obtained from the documents were supplemented using an interview with a key First Nations official. ${ }^{17,18}$

\footnotetext{
${ }^{17}$ An individual from one organization declined to be interviewed for this study despite numerous requests and follow-up correspondence.
} 
The amalgamated AFN and $\mathrm{CoO}$ approach for addressing drinking water quality issues of First Nations communities focuses on addressing gaps between the current level of financial and human resources currently available to First Nations and the financial and human resources needed for First Nations to provide quality drinking water to their communities. The idea is that First Nations require adequate funding and trained personnel to effectively build, operate, and maintain drinking water systems capable of producing a sufficient amount of quality drinking. The onus for filling the financial and human resource gap is placed on AANDC, as these organizations indicate that the federal department has not fulfilled its financial obligations nor has it provided adequate training. In addition, First Nations are unable to fill this gap within current resource levels. Recognition and respect of First Nations water rights and ideas for addressing drinking water quality issues are paramount for success.

Problem definition. The main problems according to the AFN and CoO were a lack of adequate and dedicated resources for infrastructure and human resources capacity. References to financial resources $(4.7 \%$ of $\mathrm{AFN} / \mathrm{CoO}$ factor references), funding $(2.7 \%)$, infrastructure $(3.3 \%)$, and capacity $(2.1 \%)$ were significant. Documents revealed that there is a resource gap, which is stifling adequate water quality. The interview respondent also indicated that there are inadequate resources for adequate infrastructure and O\&M (personal communication, April 4, 2012).

A second component of the problem definition is a lack of comprehensive legislation and regulations regarding the roles and responsibilities of governance actors. References to legislation $(6.2 \%$ of factor references) and regulation $(8.3 \%)$ were

\footnotetext{
${ }^{18}$ Four other First Nations Political-Territorial Organizations were contacted to participate in lieu of the non-participating organization; however, none agreed to be interviewed for the purposes of this study.
} 
significant, but these groups oppose the way the proposed legislation (Bill S-8) has been drafted. First Nation organizations have called for engagement throughout the entire legislative process, from conception to implementation as illustrated by AFN National Chief, Shawn A-in-Chut Atleo:

There is need to address the resource and infrastructure gaps that exist in First Nations communities first. The lack of financial resources is a major underlying problem, as is the related issue of capacity; [First Nations people also] agree with the need for clear standards; however, the unilateral imposition of a regulatory regime, without the agreement and cooperation of First Nations communities will inevitably result in failure (AFN, 2010a)

Theory of change. On one hand, the AFN is a national advocacy organization for First Nations in Canada. The AFN:

is designed to present the views of the various First Nations through their leaders in areas such as: Aboriginal and Treaty Rights, Economic Development, Education, Languages and Literacy, Health, Housing, Social Development, Justice, Taxation, Land Claims, Environment, and a whole array of issues that are of common concern which arise from time to time" (AFN, 2012a)

In relation to drinking water, the AFN "advocates and supports First Nations positions where the control and access to adequate water resources threatens First Nations rights and jurisdiction" (AFN, 2012b). Further, the AFN advocates for the federal government to increase "capital resources, training and management capacity to address the growing backlog in First Nations infrastructure" (AFN, 2012b). The AFN's change theory is to move First Nations water rights and drinking water quality to a point they can sufficiently operate and maintain their drinking water systems while maintaining their rights to manage these resources in their own way.

The $\mathrm{CoO}$ is a provincial political territorial organization responsible for " 133 First Nation communities located within the boundaries of the Province of Ontario" (CoO, 2012a). Its purpose "is to enable the political leadership to discuss regional, provincial 
and national priorities affecting First Nation people in Ontario and to provide a unified voice on these issues" $(\mathrm{CoO}, 2012 \mathrm{~b})$.

The overall AFN, CoO change theory is built on several activities including: garnering respect of First Nations drinking water rights; using expert advice in the development of federal government policies; ensuring that infrastructure investments are prioritized; and defining and clarifying governance actors' roles and responsibilities. First, the change theory begins with raising awareness of First Nations rights to selfgovern, including the right to manage their waters $(\mathrm{CoO}, 2010)$. As such, this theory of change assumes that First Nations are engaged in all federal government policies. Second, the change theory promotes the use of expert advice in federal government policy by using such documents as the National Assessment by Neegan Burnside and the Report of the Expert Panel on First Nations Drinking Water. The organizations express dissatisfaction with the federal government's use of these documents, particularly when weighing the high level of financial and human resources that went into their development. Third, infrastructure investments must be prioritized based on the Neegan Burnside national assessment in that systems at high-risk for contamination will first be addressed, followed by medium- and low-risk systems, so that all First Nations have equal access to clean drinking water in the shortest time period possible. Finally, the theory recognizes that governance actors' roles and responsibilities must be articulated clearly. This idea is vital to ensure that the liability of providing safe drinking water to First Nations is not offloaded to First Nations governments.

Change model. The AFN and CoO's change model comprises eight activities expressed as key steps (see Figure 3.28). 
Figure 3.2.8: AFN and CofO Change Model

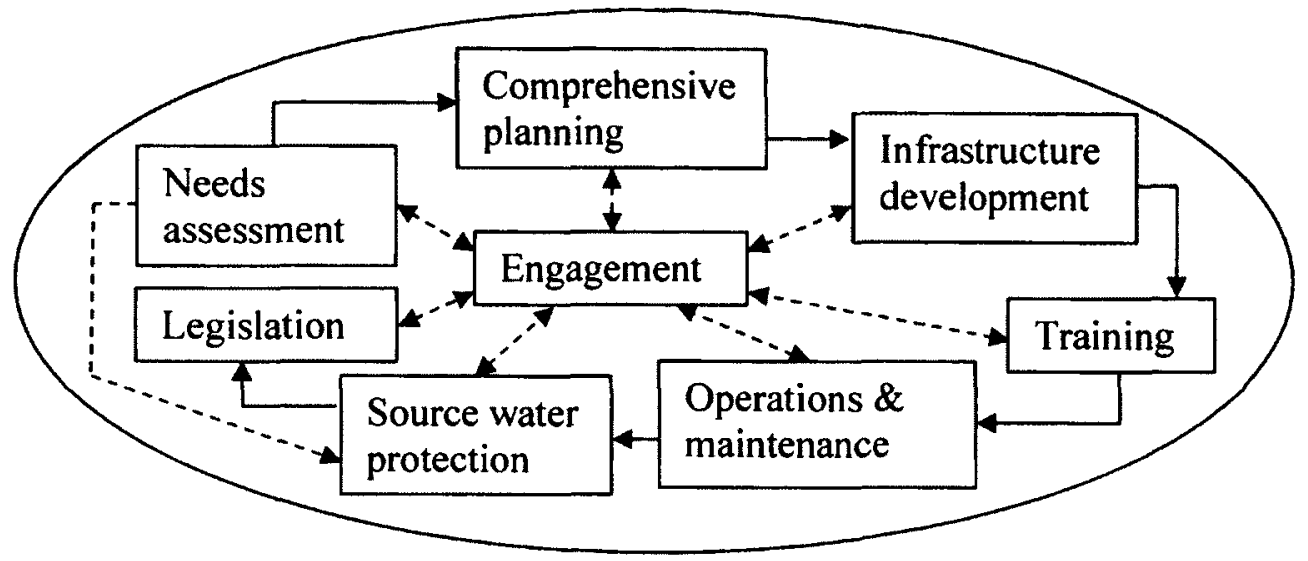

The first step, engagement, is connected to all other elements. First Nations must be adequately engaged throughout any and all decision making processes, as the Crown has the "fiduciary obligations to consult with and accommodate First Nations" (CoO, 2009; Delgamuukw v. British Columbia, 1997). The AFN (2009) suggests that this obligation extends to all federal government decisions "regarding all uses of First Nation federal lands and waters" and that the Crown has failed to reasonably fulfill these responsibilities to date. Consultation (6.4\% of factor references) was cited as a key part of engagement throughout the documents reviewed and interviews with respondents, while similar references to engagement (3.1\%), involvement (1.5\%), and accommodation (1.2\%) were also apparent. It was indicated that "First Nations communities have a clear understanding of the real needs and challenges in delivering safe drinking water" (AFN, $2010 \mathrm{~b})$ and these organizations believe "increased involvement will increase the likelihood of success of new initiatives" (AFN, 2008a). Table 27 summarizes the AFN and CoO's action theory for engagement. 
Table 27. AFN and CoO Action Theory: Engagement

\begin{tabular}{ll}
\hline Action Theory Component & Required Inputs \\
\hline a) Resources & - None specified \\
b) Implementing Organizations & - AANDC, HC, EC, First Nations \\
c) Implementers & - Ministers, bureaucrats, senators, C\&Cs \\
d) Associates/Partners & - First Nations groups \\
e) Ecological Context & - Engagement to-date has been insufficient \\
f) Intervention Protocols & - AFN and CoO proposed engagement/consultation processes \\
g) Target Population & - First Nations community residents, C\&Cs
\end{tabular}

Next, if engagement occurs, then steps must be taken to assess current infrastructure and human resource needs. Terms pertaining to assessment $(1.1 \%$ of factor references) and evaluation ( $0.9 \%)$ reveal that a needs assessment will enable an estimate of environmental protection and the resource gap, including costs associated with infrastructure, human resources, and training. Documents indicate that there must first be a "clear picture of the infrastructure needs in First Nation communities" (CoO, 2009) before such needs can be addressed, assuming there is sufficient funding disseminated for "each region to conduct a thorough impact analysis to determine the financial, technical, and policy development needs for each region" (AFN, 2010c). Table 28 provides a summary of the AFN and CoO's action theory of needs assessment. 
Table 28. AFN and CoO Action Theory: Needs Assessments

\begin{tabular}{ll}
\hline Action Theory Component & Required Inputs \\
\hline a) Resources & $\begin{array}{l}\text { - } \$ 9.3 \mathrm{M} \text { for the Neegan Burnside assessment, assessments and } \\
\text { evaluations from unspecified amount of recommended by } \\
\text { Neegan Burnside }(201 \mathrm{~b}), \text { totalling } \$ 63.4 \mathrm{M}\end{array}$ \\
$\begin{array}{ll}\text { b) Implementing Organizations } & \text { - AANDC, HC, EC } \\
\text { c) Implementers } & \text { - A variety of potential implementers } \\
\text { d) Associates/Partners } & \text { - C\&Cs, First Nations groups, universities, contracted firms } \\
\text { e) Ecological Context } & \text { - Some assessments have occurred, more required } \\
\text { f) Intervention Protocols } & \text { - None specified } \\
\text { g) Target Population } & \text { - C\&Cs, federal government }\end{array}$
\end{tabular}

Next, it follows that if needs assessments are carried out then the four governance actors can develop a comprehensive plan of action for addressing them. Planning $(2.6 \%$ of factor references) for fulfilling identified needs was a common theme, including a desire for the Minister of AANDC to "work collaboratively with First Nations communities to put forth a clear written plan" to address the resource gap (AFN, 2010d). This plan must consider the ability to monitor progress. The AFN indicates it has "received little information regarding how the $\$ 1.8$ billion [approaching $\$ 2.5$ billion] to address First Nations safe drinking water needs has been spent" (AFN, 2008b). Table 29 summarizes the action theory for comprehensive planning as a step within the AFN and CoO theory of change. 
Table 29. AFN and CoO Action Theory: Comprehensive Planning

\begin{tabular}{|c|c|}
\hline Action Theory Component & Required Inputs \\
\hline a) Resources & $\begin{array}{l}\text { - Unspecified amount from } \$ 63.4 \mathrm{M} \text { recommended by Neegan } \\
\text { Burnside }(2011 \mathrm{~b})\end{array}$ \\
\hline b) Implementing Organizations & - AANDC, HC, EC, First Nations \\
\hline c) Implementers & - Departmental policy drafters, C\&Cs \\
\hline d) Associates/Partners & - Expert organizations, contracted firms, First Nations groups \\
\hline e) Ecological Context & - Some planning has occurred, lacked performance indicators \\
\hline f) Intervention Protocols & - None specified \\
\hline g) Target Population & - Budget drafters \\
\hline
\end{tabular}

Next, if a comprehensive plan is developed for addressing the resource gaps identified, it follows that that plan could then be implemented beginning with infrastructure development (3.3\% of factor references), and investments in capital $(1.7 \%)$. That is "capacity needs are not isolated to the requirement for trained water system operators but the larger need is for adequate water systems infrastructure" (AFN, 2008a), but that "there is need to address the resource and infrastructure gaps that exist in First Nations communities first" (CoO, 2008a; personal communication, April 4, 2012). Table 30 summarizes the AFN and $\mathrm{CoO}$ action theory for infrastructure development.

\section{Table 30. AFN and CoO Action Theory: Infrastructure Development}

\begin{tabular}{ll}
\hline Action Theory Component & Required Inputs \\
\hline a) Resources & - Estimated \$738M by Neegan Burnside (2011b) \\
b) Implementing Organizations & - AANDC, First Nations \\
c) Implementers & - Contractors \\
d) Associates/Partners & - HC, PWGSC, CMHC, First Nations groups \\
e) Ecological Context & - There is an apparent resource gap \\
f) Intervention Protocols & - Details in funding agreements \\
g) Target Population & - First Nations community residents
\end{tabular}

Next, if infrastructure needs are met, First Nations communities will require the capacity to fully operate water systems. First Nations must be introduced to sufficient 
information and tools to operate and maintain their infrastructure, including training ( $2.1 \%$ of factor references) for WTOs, as "training of plant operators becomes more and more important in order to ensure quality/assurance/control" (AFN, 2010e). Also "the technical support available to First Nations, such as training in operations and maintenance is inadequate" $(\mathrm{CoO}, 2005)$, as First Nations operators are lagging behind their non-First Nations counterparts because $60 \%$ of First Nation communities employ a WTO with any level of training. There is need to "provide adequate training, education, and resources for First Nation leaders to enable them to take ownership of potential improved and effective infrastructure on reserves" (AFN, 2010b). The action theory for training according to the AFN and $\mathrm{CoO}$ is summarized in Table 31.

\section{Table 31. AFN and CoO Action Theory: Training}

Action Theory Component Required Inputs

a) Resources

- \$10M per year from AANDC funding plus additional unspecified amount from $\$ 63.4 \mathrm{M}$ recommended by Neegan Burnside (2011b)

b) Implementing Organizations

- Circuit Rider Training organizations, provinces

c) Implementers

- Circuit rider trainers

d) Associates/Partners

e) Ecological Context

- AANDC, HC, other treatment organizations, C\&Cs, First Nations groups

f) Intervention Protocols

- Only $40 \%$ of First Nations' WTOs have any certification

g) Target Population

- The Protocol, GCDWQ, provincial legislation

- WTOs

Next, if training needs are met, First Nations communities will have the tools to effectively operate ( $2.8 \%$ of factor references) and maintain $(1.8 \%)$ their water systems. However, sufficient funding will be necessary to adequately fulfill standardized O\&M activities. Such activities are specific and central to the treatment method, which is tied to water source: standardized activities are listed within the Protocol. Approximately $40 \%$ of current O\&M budgets are covered by AANDC funding agreements, but First Nations 
express concern about this given that it is the federal department's responsibility to provide $80 \%$ of O\&M funding (personal communication, April 4,2012 ). It is acknowledged that the funding formula for AANDC O\&M contributions are outdated and do not accurately reflect current needs of communities. In fact, some communities are not even allocated sufficient funding to obtain adequate chemicals for treatment, nor are they able to allocate sufficient funds to cover the unfunded proportion (20\%) of O\&M for which they are solely responsible (AFN \& $\mathrm{CoO}$ interview respondent, personal communication, April 4, 2012). Table 32 summarizes the AFN and $\mathrm{CoO}$ action theory of O\&M.

Table 32. AFN and CoO Action Theory: Operations and Maintenance

Action Theory Component Required Inputs

a) Resources

- $80 \%$ funded by AANDC, $20 \%$ by First Nations; $\$ 431 \mathrm{M}$ between 2006-10, unspecified amount from $\$ 330 \mathrm{M}$ from biannual A-Base allocations from 2008-14, $\$ 40 \mathrm{M}$ in additional departmental allocations per year, additional 14.7M required according to Neegan Burnside (2011 b)

b) Implementing Organizations

- First Nations, MTA municipalities

c) Implementers

- WTOs

d) Associates/Partners

- C\&Cs, Circuit rider trainers, AANDC, HC, First Nations groups

e) Ecological Context

- AANDC funds not equivalent to $80 \%$, First Nations have difficulty providing $20 \%$

f) Intervention Protocols - The Protocol

g) Target Population

- First Nations community residents

Next, if O\&M activities occur and are funded as needed, focus can be placed on other challenges such as source water protection. The need to protect $(3.3 \%$ of factor references) the environment and/or the source water (4.4\%) was significant. It was explained that the "ability to provide safe drinking water depends upon a healthy environment and clean sources of water" (AFN, 2009a). Furthermore, these documents 
suggest that source water protection is "a key requirement in developing a long-term, effective, sustainable solution" and that without it First Nations "will be in a game of catch-up, trying to find better technology to clean polluted water" (AFN, 2008b; emphasis added). Planning is significant because First Nations groups suggest that it has typically been offloaded to the First Nations without resources beyond those provided for EC to develop tools (personal communication, April 4, 2012). Table 33 summarizes the action theory for source water protection according to the AFN and $\mathrm{CoO}$.

\section{Table 33. AFN and CoO Action Theory: Source Water Protection}

\begin{tabular}{ll}
\hline Action Theory Component & Required Inputs \\
\hline a) Resources & - Unspecified amount from $\$ 63.4 \mathrm{M}$ recommended by Neegan \\
& Burnside $(2011 \mathrm{~b})$ \\
b) Implementing Organizations & - First Nations, EC \\
c) Implementers & - C\&Cs \\
d) Associates/Partners & - Watershed protection associations, provinces \\
e) Ecological Context & - Funding is not currently provided by federal actors \\
f) Intervention Protocols & - None specified \\
g) Target Population & - First Nations community residents \\
\hline
\end{tabular}

Next, once the infrastructure, training, $O \& M$, and source protection gaps have been closed, it follows that First Nations communities will be able to adhere to a comprehensive legislative framework (6.2\% of factor references) and subsequent regulatory regime $(8.1 \%)$. There is an urgent need to first address all underlying drinking water quality issues in First Nations communities before preparing regulations. The action theory for drinking water quality legislation according to the AFN and $\mathrm{CoO}$ is summarized in Table 34. 
Table 34. AFN and CoO Action Theory: Legislation

\begin{tabular}{ll}
\hline Action Theory Component & Required Inputs \\
\hline a) Resources & - None specified \\
b) Implementing Organizations & - First Nations, EC, HC, AANDC \\
c) Implementers & - Departmental policy drafters, C\&Cs \\
d) Associates/Partners & - Experts, First Nations groups \\
e) Ecological Context & - Bill S-8 may receive Royal Assent \\
f) Intervention Protocols & - AFN's proposed policy development process \\
g) Target Population & - First Nations community residents \\
\hline
\end{tabular}

The AFN has established a five-step approach for developing drinking water policies, which have been shared with AANDC. Figure 6 provides a summary of this proposed process, which includes active engagement and First Nations participation throughout. Regulations would take the form of a comprehensive set of activities and objectives that all governance actors agree to. These regulations would be enforced by a regulatory body, preferably a newly established water commission with representation from First Nations across Canada.

Figure 4. AFN's Proposed Policy Development Process

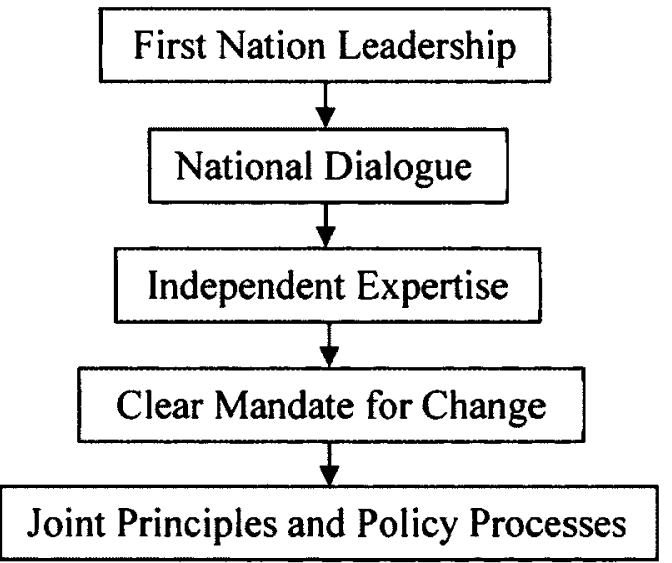




\section{Comparison of Governance Actors' Approaches}

The purpose of this section is: to assess stated problem definitions; to compare foundational principles of the governance actors' change theories; and to compare and contrast the most important activities that relate to each governance actor's theory of change. Comparisons of specific steps are first ordered in the most common placement of those steps. For example, a comparison of the actors' perspectives on legislation occur last, as this step is identified last in HC and EC's change theories even though AANDC includes regulation as a middle step of its theory. In the event that there is no clear majority, steps are placed in the order that water flows. For example, water is first untreated source water (protection), then moves into the treatment facility (O\&M), where it is treated (training) and distributed (monitoring).

Each step is summarized using characteristics of each actor's description of the activity, followed by an analysis and identification of a primary gap resulting from differing governance actors' perspectives on that activity.

Comparison of governance actors' problem definitions. The four actors understand the problem of drinking water quality in First Nations communities differently; however, general trends can be discerned. These four actors illustrated two ways in which such problem definitions were conveyed. First, EC understands the problem as improving water quality, primarily through source water protection. For EC, it would be ideal if water were clean before drinking water treatment processes were instituted. Second, the other actors define their problem definitions in a way that makes the achievement of objectives contingent on the mandates of other actors. For example, although $\mathrm{HC}$ greatly recognizes the need for improving water quality monitoring, its 
achievement of higher drinking water quality depends on proper treatment (First Nations responsibilities) and the financial and human resources to ensure proper treatment (AANDC and First Nations responsibilities). Likewise, AANDC understands the problem as inherent difficulties with the operation and maintenance of facilities (First Nations responsibility). AANDC indicates that capital investments (AANDC responsibilities) "seem like the most obvious solution" but only account for $30 \%$ of the underlying issues (AANDC, 2011d). AANDC also indicates that it supplies $80 \%$ of the funding for O\&M, and that First Nations often do not cover their required $20 \%$ of these costs. AANDC emphasizes a need for ensuring that operators are trained (First Nation responsibility via funding agreements) and that the department provides approximately $\$ 10$ million annually for this purpose (AANDC, $201 \mathrm{lg}$ ). Finally, the AFN and $\mathrm{CoO}$ explain that current funding for capital and capacity investments (AANDC responsibility) is the main problem resulting in First Nations drinking water quality issues. The claim here is that First Nations communities would be able to operate and maintain drinking water systems effectively if they received adequate funding for proper infrastructure, personnel training and subsequent compensation, and all O\&M activities. Indeed, the AFN and $\mathrm{CoO}$ suggest that AANDC overstates its financial contributions.

These four problem definitions illustrate that the four actors do not share a common perspective or mandate regarding the poor drinking water quality in First Nations communities. Three of the actors explain that the results of their activities cannot be maximized without appropriate reliance on the other actors. The one outlier, EC, identifies its own perceived role as the primary issue: it explains that there is a gap with regard to its own mandate, which is unfunded and sometimes considered an afterthought. 
Comparison of governance actors' theories of change. The four governance actors hold distinct broad theories of change. AANDC's mission is to help First Nations become self-sustaining and self-reliant. In this regard, they will be able to operate and maintain water systems using funds from their own economies (and presumably allocated funds). The AFN and CoO's change theories focus on obtaining and retaining sufficient funds in order to protect their water rights. There is a gap between the AANDC and the AFN and CoO's change theories in that funding for responsibilities is contingent on each other's activities. HC's responsibility is to design and implement the appropriate activities and procedures (i.e., monitoring) for preventing health related drinking water problems from occurring, by mitigating against human and/or machine error. EC's responsibility focuses on preventing source water contamination. The responsibilities of these actors are different, which leads to some interesting dynamics.

Engagement. Engagement is perhaps the most important activity that is shared by all four actors. The AFN and $\mathrm{CoO}$ suggest that First Nations be engaged, but that they should be much more than consulted. HC's view is congruous with the AFN and CoO, and suggests that First Nations involvement is crucial to any activity's success. AANDC and EC both suggest that involvement of First Nations should occur for specific activities only. For example, AANDC's proposed legislative process (see Figure 1) begins with an apparent unilateral process followed by feedback. This method of engagement is incongruent with AFN's proposed legislative process (see Figure 6), which requires First Nations involvement for developing legislation. AANDC's process was utilized for Bill S-11, which is highly contested by First Nations. EC's suggested engagement goes beyond engaging First Nations to include all Canadians in watershed protection. Overall, 
there is a gap regarding the amount of First Nation engagement necessary for successful implementation of water policy.

An activity related to engagement is raising awareness and increasing the education of First Nations with regard to drinking water quality. The idea is that First Nations will be better able to understand the importance of clean drinking water, which will translate into sufficient resourcing and water treatment within the communities. This education will help First Nations become more confident in using their treatment processes and prevent communities from using raw source water. For the AFN and CoO, education and awareness will help bring First Nations into alignment with other actors' knowledge and understanding of drinking water systems.

Another activity related to engagement is recognizing and respecting treaty rights, as outlined by the AFN and CoO. AANDC describes transfer payments to First Nations as funds "for the delivery of programs and services that would ordinarily be provided by various levels of government (provincial, territorial, municipal)" (AANDC, 2010). Thus it does not appear that the First Nations band councils are recognized as a legitimate form of government. Addressing this perception may be the key to fully engaging First Nations. Documents pertaining to incorporating traditional knowledge indicate that First Nations hold special knowledge regarding how to manage and respect the lands and waters within their territories. These differences may be central to differences between the federal government and First Nations responsibilities. There appears to be a significant gap with respect to the recognition of and value placed on treaty rights and traditional knowledge. Indeed, without incorporating these rights and traditional knowledge in their responsibilities, First Nations may similarly overlook federal 
departmental knowledge within their communities. That is, First Nations may not show deference for the federal governance actors' protocols and guidelines if their own rights and knowledge are not respected in kind.

Roles and responsibilities. The actor's roles are lacking clarity around expectations. AANDC recognizes its role to fund First Nations, but the level of funding required remains unclear. Band councils require clearer lines of accountability to both AANDC and First Nations residents with regard to funding managed. Actors within First Nations, such as WTOs, CBWMs, and band councils must also have clearly defined roles and responsibilities to address their responsibilities. Likewise, due to the convoluted nature of the prescribed protocols, manuals, and guidelines, roles with regard to monitoring are sometimes blurred. Finally, EC's role is to protect water sources, but its participation level in this activity is not clear to all actors. With such ambiguity, it is likely that unassigned activities will go unfulfilled, as the mentality presented in the problem definitions is to defer to other governance actors rather than voluntarily fill the void resulting in poor drinking water quality for First Nations.

Comparison of governance actors' change models. Research. The first activity among the change theories is research. $\mathrm{HC}$ advocates for more research with regard to WDEs, which is in line with the department's mandate. By contrast EC suggests that research should investigate how water usage connects with the ecosystem, contamination, and human impact on the environment. EC's version of research is also in line with its own mandate. The AFN and $\mathrm{CoO}$ view research in terms of needs assessment so as to determine the funding needed for water infrastructure and capacity building (including personnel training and $O \& M$ funding). These groups suggest that expert research and 
analysis should inform policy decisions. Finally, notions of research for AANDC also include a needs assessment, but articulate that this work has mostly occurred. The primary difference between AANDC and the AFN and $\mathrm{CoO}$ is with regard to the extent to which needs assessments are used. For example, the AFN and $\mathrm{CoO}$ articulate that all funding should reflect the results of the National Assessment of First Nations Water and Wastewater Systems, whereas AANDC states that "this information will be an important resource as the department works with its partners and stakeholders to determine future directions", leaving the impression that it will not be held as whole truth. The gap prevalent throughout this step is twofold. First, there are three distinct types of research recommended within the four change theories, suggesting disagreement between the actors regarding the most important form of research. Second, AANDC and the AFN and $\mathrm{CoO}$ differ with regard to the extent to which such research should be utilized.

Planning. The next activity is comprehensive planning, which AANDC references more than any other actor, and articulates the need to incorporate research within planning processes. The AFN and $\mathrm{CoO}$ also integrate research into planning, but aim this activity at addressing resource gaps. AANDC and these First Nations groups each suggest that prioritization in the planning process must emphasize high-risk communities, followed by medium- and subsequently low-risk. Risk ratings referred to by these two actors are those identified in the National Assessment of Water and Wastewater Systems in First Nations Communities. EC's approach to planning emphasizes water protection and sustainable use, and like AANDC and the First Nations actors, stresses the importance of incorporating research in the planning process. Finally, HC's discussion of planning emphasizes the development of manuals and procedures for 
First Nations' and federal personnel. Such tools are generally within the scope of HC's mandate (e.g., monitoring). The gap present in this activity is defining what to plan. Each actor recommends using the aforementioned research, but three distinct directions of planning are emphasized. Further, there is disagreement on the scope of planning activities between $\mathrm{AANDC}$ and the $\mathrm{AFN}$ and $\mathrm{CoO}$ (i.e., resource planning versus whole change theory planning).

Source water protection. Source water protection is a key activity for each of the governance actors. However, the importance attached to this activity differs between them. EC advocates that water source protection should be the most important step in remedying issues of drinking water quality, whereas the AFN and $\mathrm{CoO}$ attach less importance to this activity. These organizations stress the importance of this activity insofar as it is congruent with many First Nations' cultural beliefs. In fact, they stress that it is a responsibility bestowed on First Nations by the creator. On the other end of the spectrum, AANDC weights 10 percent of contamination risk to water source (with only five percent variability). Similarly, $\mathrm{HC}$ includes source water protection in its change theory, but emphasizes it as an underlying assumption. That is, source water protection is part of any multi-barrier approach, but is less prominent in the change theory than other actors. The most prominent gap relating to source water protection is funding support. That is, there is disagreement in the importance of this activity between the governance actors, resulting in inconsistent funding.

Infrastructure investment. Investment in infrastructure is a high priority for all actors. AANDC supports infrastructure investment in treatment plants, distribution piping, and other capital expenditures, prioritizing from high- to low-risk communities. 
The AFN and $\mathrm{CoO}$ maintain that infrastructure investment is part of the resource deficit, and that increased funding for infrastructure is required from AANDC. EC also supports investments in infrastructure. The one outlier regarding infrastructure investments is $\mathrm{HC}$, which suggests that there is a need to ensure infrastructure is available, but also cautions against building facilities with technologies beyond the capacity of First Nations communities. Thus, HC suggests that the tendency to 'overbuild' has contributed to drinking water issues.

Two gaps are observed in this activity: there is disagreement in the amount of funding that AANDC should allocate; and $\mathrm{HC}$ does not support simply building new infrastructure, but first recommends addressing training on technologies that First Nations are able to use. This gap is challenging because First Nations are responsible for drafting water infrastructure plans, $\mathrm{HC}$ engineers make assessments from a health perspective of water system design, and AANDC provides funds to First Nations for these infrastructure plans.

Training. Training First Nations individuals to successfully utilize water infrastructure is supported by all actors, especially to educate WTOs. The AFN and CoO maintain that training should include band councils on the importance of and procedures for allocating sufficient funds (the required 20\%) for O\&M of water facilities. These ideas are corroborated by the AANDC interview respondent, who indicated that band councils do not always provide funding necessary for O\&M. HC and EC both substantiate this need and understand the challenges of keeping WTOs within the communities. AANDC provides only $\$ 10$ million annually in training funding for all 
First Nations, while funding agreements for O\&M are tied to band councils' ensuring that WTOs are certified (AANDC, 2011g).

Operations and maintenance. Each actor highlights the importance of proper O\&M of drinking water systems. Both AANDC and $\mathrm{HC}$ regard this activity as the primary problem. They rely heavily on the use of protocols, procedures, and guidelines for ensuring that drinking water is treated with the appropriate type and amount of chemicals. The primary issue EC associates with O\&M is compensating and retaining WTOs. Finally, the AFN and CoO corroborate EC's assessment of O\&M but further articulate that funding resources provided by AANDC are not on par with that which was promised ( $80 \%$ of the total). Overall, the gap prevalent in this step is the prescribed amount of funding for day-to-day O\&M.

Monitoring. Three of the four actors emphasize monitoring as a key activity in their change theories. EC specifies that monitoring practices should occur prior to entering the drinking water system for contamination. AANDC's emphasis on monitoring appears after regulations have been set in place to ensure compliance with O\&M best practices. HC suggests that monitoring should occur prior to entering and throughout the water system, observing the quality of water throughout each step in the water treatment process in order to identify and mitigate specific issues as they arise. The gap of this activity is defining the role water quality monitoring practices should fulfill. Establishing whether monitoring is meant to catch contamination issues, water treatment errors, or regulatory compliance will greatly influence the extent to which First Nations carry out this activity. At present, First Nations place little emphasis on monitoring perhaps as a result of the confusion over its purpose. 
Legislation, regulation, and enforcement. Perhaps the most contentious issue is legislation, regulation, and enforcement, which all actors include as an activity in their change theories. Three of the four ( $\mathrm{HC}, \mathrm{EC}, \mathrm{AFN} \& \mathrm{CoO})$ suggest that this should be the last step in any change process. The rationale is that enforcing water regulations prior to addressing the other deficiencies would set up First Nations to fail under any new regulatory framework. By contrast, AANDC places this step as a sequential set of steps in the middle of its change theory. AANDC'S rationale for this placement is to ensure that subsequent change theory steps are strictly adhered to. Overall, the gap in incorporating regulation is determining the most effective regulations, and when they ought to be applied.

\section{Conclusion}

Each governance actor has presented a unique problem definition and theory of change, which has likely had an impact on the direct oversight of drinking water quality in First Nations communities. Differences in theories and approach likely result in inconsistent adherence to any one set of responsibilities. Such inconsistent adherence can then be used as evidence for any one actor to impose their responsibilities at any given time. In effect, confusion can only result in the following ways.

First, there are clear differences in terms of understanding the problem which results from placing the onus of drinking water issues on other actors (or an activity for which one has limited responsibility), and which forces community level actors to look for support in multiple places. For example, if community level actors request additional O\&M funding from their band council, the band council will refer them to AANDC. In turn, AANDC will suggest that the band council already has sufficient funding that is not 
being used efficiently. The result can be insufficient funding for O\&M. Thus, gaps in problem definition can be manifested in making the problems of water quality worse, rather than coming to agreement on the problems to be addressed.

Second, differences in change theories and activities can be felt in how communities understand and address their own water management. That is, each actor suggests that role definition is crucial for addressing First Nations' drinking water quality. Although role definition is likely an underlying problem in any community, it is a particular problem for First Nations as ambiguity with regard to any specific task or funding is likely to lead to finger pointing to other actors (see problem definitions), rather than the local responsible party voluntarily filling a void. Further, a lack of engagement, and disagreement regarding engagement, is only likely to exacerbate issues of role definition, as any present roles and responsibilities currently in place have been 'assigned' rather than agreed upon with First Nations. Therefore, it is likely that the negativity associated with the alleged unilateral assignment of roles within new legislation will further discourage a First Nations actor from voluntarily filling a void.

Finally, different interventions will cause confusion at the local level. Specifically, major steps such as source water protection will likely be underfunded, understaffed, and under-supported at the community level due to similar (and apparent and perceived) disinterest by the governance actors regarding these activities. Although governance actors have articulated the importance of these activities, without support, First Nations actors will not see the value or rationale for completing them.

In the chapters that follow three match-paired case studies identify issues apparent in First Nations communities' water systems relative to analogous municipalities. These 
comparisons are made first to determine whether drinking water in First Nations communities is of poorer quality than analogous non-First Nations communities, as stated by a variety of experts (Justice O'Connor, 2002b; St. Germain, 2007; Swain, Louttit, \& Hrudey, 2006). Second, these match-paired case studies are conducted to determine if any disparity between drinking water in these communities reflect any or all of the identified problem areas. The idea is that confusion between the way the governance actors institute their activities for addressing First Nations drinking water quality issues may actually exacerbate problems, rather than resolve ongoing persistent problems of water quality. 


\section{Chapter 4: A Comparison of Communities using the Same Drinking Water System}

The following chapter is the first of the three matched-pair case studies. This chapter compares and contrasts two communities which rely on the same drinking water system (i.e., drinking water source, treatment facility, and the majority of the same distributions system), Longlac Ontario and Ginoogaming First Nation. These communities are analogous in that they are similarly located, have a similar population size, and use the same drinking water source. First, Longlac is 30 kilometres east of Geraldton, Ontario (Greenstone, 2012a), and Ginoogaming is located five kilometres east of Longlac (Salma, 2010). Second, Longlac's population of 1,388 (Statistics Canada, 2012g) is comparable to Ginoogaming's 167 person population (Statistics Canada, 2012h). Finally, both communities draw their drinking water from Long Lake, a surface water source. Longlac supplies drinking water to Ginoogaming through a municipal transfer agreement (MTA). Specifically, Ginoogaming pays Longlac to operate and maintain the drinking water system and deliver water to the First Nation's territorial border. Ginoogaming is then responsible for the section of the distribution within its land.

Despite similarities in these three common characteristics, the Longlac and Ginoogaming differ on a number of elements throughout the oversight of their drinking water, which stem from broad divergence in governance structure. Longlac is subject to a two-tiered governance structure whereby the Ontario Ministry of the Environment (MOE), in conjunction with other provincial ministries, establishes and enforces drinking water regulations under the auspices of the Ontario Safe Drinking Water Act, 2002. Longlac's local government, is then responsible for operating and maintaining the 
drinking water system in accordance with those regulations. By contrast, Ginoogaming's drinking water is governed by four independent actors including the Ginoogaming Chief and Council, Aboriginal and Northern Development Canada (AANDC), Health Canada (HC), and Environment Canada (EC). AANDC is primarily responsible for funding activities related to drinking water infrastructure, personnel training, and $80 \%$ of operations and maintenance activities. HC is responsible for overseeing drinking water quality monitoring programs, helping to identify issues with drinking water quality, and reviewing drinking water infrastructure proposals. EC's role is to provide advice to the First Nation with regard to source water protection. Finally, Ginoogaming is responsible for the day-to-day operations and maintenance and direct oversight of the drinking water system. Ginoogaming must also fund $20 \%$ of operations and maintenance budgets. Activities related to drinking water within Ginoogaming are guided by the Protocol for Centralised Drinking Water Systems in First Nations Communities (the Protocol) and the Guidelines for Canadian Drinking Water Quality (GCDWQ). The Protocol stipulates that if the guidelines and/or regulations of a given province are stricter than the Protocol or GCDWQ, those specific provincial guidelines should direct the First Nation. Because the GCDWQ regulates more parameters than the Safe Drinking Water Act, 2002, the documents guiding Ginoogaming are more stringent than are those regulating Longlac.

There are three primary rationales for this chapter; the first of which is to investigate the differences in community-level water management and subsequent drinking water quality of two communities that share a drinking water system. Second, the chapter has been devised to explore whether any differences that have been identified between the two communities reflect the gaps in the First Nations drinking water quality 
governance actors' theories of change. Finally, this chapter is intended to explore differences in the evaluation of the same drinking water system. That is, the MOE and AANDC require different evaluations for Ontario and First Nations drinking water systems respectively. Divergence regarding system issues identified or recommended improvements will illustrate difference in evaluation. Longlac and Ginoogaming are compared across the several elements of drinking water quality including: local governance description, drinking water system design, source water protection measures, operations and maintenance $(O \& M)$ practices enlisted, measures of drinking water quality, and recommended improvements for the drinking water system.

This chapter's primary data sources are engineer reports on the drinking water system from the perspective of Longlac (MOE, 2012e) and Ginoogaming (Salma, 2010). The Longlac report was submitted to the MOE by an unidentified independent engineer as part of the requirements of the Safe Drinking Water Act, 2002. Interviews with key respondents from both Longlac and Ginoogaming were conducted to provide additional insight. The chapter is also supplemented with information drawn from the communities' websites.

\section{Community Descriptions}

Longlac. The town of Longlac was incorporated into the Municipality of Greenstone in 2001 during the provincial government's push to amalgamate townships into larger governed areas (Greenstone, 2012a). The rationale for amalgamating close in proximity townships was to pool financial resources for social goods to increase a municipal government's flexibility of resources and to capitalize on economies of scale. 
Greenstone is comprised of eight former townships including Nakina, Geraldton, Beardmore, Caramat, Jellicoe, Macdiarmid, Orient Bay, and Longlac.

Greenstone is governed by an elected mayor and council consisting of eight members, of which two are elected by Longlac residents (Greenstone, 2012b). Greenstone's public service department has three divisions, one of which is responsible for water supply and distribution among other aspects of public works (Greenstone 2012c). This department is overseen by the Director of Public Services. A Manager of Public Works oversees tasks related to infrastructure of water supply and distribution systems (Greenstone 2012d). The number of employees within the public works division was not specified. While the municipality governs infrastructure spending regarding water systems, O\&M of water its water systems have been contracted with the Ontario Clean Water Association (OCWA).

The OCWA is a Crown Agency in Ontario that operates and maintains approximately 180 water facilities throughout the province (OCWA, 2012c). As part of its agreement with Greenstone, the OCWA operates and maintains the water treatment system at Longlac. The OCWA designates three WTOs to the Longlac drinking water treatment plant (personal communication, April 23, 2012). The senior operator in charge of supervising the other WTOs has two years of experience and is trained to a Level I certification for both treatment and distribution systems. One secondary operator has five years of experience and has obtained Level II certifications for both the treatment and distribution systems. The other secondary WTO has two years of experience and is trained to the first certification level of both distribution and treatment processes. The plant also has access to a regional WTO that has obtained certification Levels of III for 
treatment and II for distribution. The Longlac treatment facility has a sufficient number of trained WTOs to effectively operate the water system (MOE, 2012e).

A breakdown of financial resources allocated to Longlac's drinking water system was not made available. The detail regarding the funds the community of Longlac transfers to the OCWA for O\&M services was similarly not disclosed. The Municipality of Greenstone charges residents an annual $\$ 563.76$ flat rate for water services, whereas industrial and commercial users are charged $\$ 2.04 / \mathrm{m}^{3}$ (Greenstone, 2012e). Interestingly, Greenstone charges the First Nations it services, Ginoogaming and Long Lake 58 First Nation, user fees of $\$ 2.69 / \mathrm{m}^{3}$ (Salma, 2010).

Ginoogaming First Nation. Ginoogaming is governed by a Chief and six Council members that are elected through the two year cycle electoral process enlisted in the Indian Act, 1985 (Ginoogaming, 2012a). The band employs eight administrators, one of which is responsible for community O\&M (Ginoogaming, 2012b). Three individuals are also responsible for economic development. Together with the Chief and Council these four individuals are the primary staff that oversees activities related to drinking water quality management, procurement, and water infrastructure within the community. As noted, drinking water treatment is provided through an MTA with Longlac. Thus drinking water services are ultimately provided by the individuals discussed in the Longlac community description, including OCWA employees. However, Ginoogaming's band administration is responsible for all activities related to distribution within the community's territorial border (Salma, 2010). For O\&M practices, Ginoogaming employs one primary and one secondary WTO to operate and maintain the distribution system (Salma, 2010). Neither of WTO is trained to any distribution or treatment 
certification level; however, the primary operator has multiple years of experience and has enrolled in a Level I distribution certification course.

Ginoogaming is reliant on AANDC transfers for funding drinking water infrastructure and O\&M costs. AANDC currently provides $\$ 153,033$ per year for water and wastewater systems. Salma (2010) estimates that the First Nation needs approximately $\$ 42,500$ more per annum to sufficiently finance the $O \& M$ costs provided by Longlac, at $\$ 2.69 / \mathrm{m}^{3} .19$

Comparison of community descriptions. There are notable differences between the management of Longlac and Ginoogaming's drinking water quality including funding management and distribution management. First, Longlac relies primarily on user-fees to fund drinking water quality management. Therefore, there is a significant pool of resources from a total population of 5,258 residents plus industry available to fund normal operation of the system as well as fluctuations in O\&M and infrastructure costs, as needed (MOE, 2012e). By contrast, Ginoogaming relies on lump sum AANDC transfers to pay its user-fees to Greenstone and to fund activities with regard to the distribution system. These funds are insufficient to fund normal O\&M activities (Salma, 2010). As a result, the First Nation does not have the financial capacity to address issues in the distribution system when they arise. This difference reflects the divergence between governance actors' theories of change with regard to funding O\&M costs such that AANDC believes that funding would be sufficient if First Nations used transferred money more efficiently and the AFN and $\mathrm{CoO}$ suggest that they can only effectively manage drinking water systems with more funding.

\footnotetext{
19 This pricing is notable for the First Nation, as it is significantly higher than the Canadian average of $\$ 1.84 / \mathrm{m}^{3}(\mathrm{EC}, 2011)$.
} 
The second difference is that Longlac outsources the operations, maintenance, and infrastructure development of its distribution system, whereas Ginoogaming oversees the management of the distribution system within its borders. This difference is pertinent to drinking water quality, as Longlac's distribution system is overseen by an organization that is dedicated to drinking water quality expertise, whereas Ginoogaming's WTOs that oversee distribution are untrained. Therefore, Longlac is better equipped to effectively manage its distribution system than is Ginoogaming, including human resource capacity to adhere to the presiding regulations and/or guidelines/protocol. This difference is again likely a reflection of the governance actors' change theories, with specific regard to funding. AANDC is responsible to fund WTO training, while Ginoogaming is required to ensure its WTOs are adequately trained. However, AANDC's funding for First Nation WTO training is on a national basis and is through a variety of partnerships, which can be costly relative to the amount invested per year ( $\$ 10$ million). AANDC is also able to withhold transfer payments for drinking water systems for First Nations who do not have adequately trained WTOs in place; however, this would only further detriment Ginoogaming with regard to water infrastructure and/or O\&M funds. Further, if these WTOs are sufficiently trained their employability would increase, causing market forces to raise their expected real wages. With Ginoogaming already impaired with regard to O\&M funding, and no legitimate WTO training enforcement mechanisms, there is disincentive to ensure WTOs are trained. Therefore, with opposed change theories in relation to funding, AANDC and the AFN and $\mathrm{CoO}$ are enabling these training issues to persist. 
Despite these differences, similarities between Longlac and Ginoogaming with regard to local government are apparent as both are represented by a democratically elected head and council. The electoral process is relevant to drinking water quality management, as, in theory, these elected officials represent the will of the people. The idea here is that these communities are able to replace the head and council should they be dissatisfied with the management of drinking water quality. Longlac's electoral cycle is longer than is Ginoogaming's; therefore, the First Nation has greater opportunity to replace the head and council than does Longlac. Further, only $20 \%$ of the councillors at Greenstone represent Longlac whereas all six Ginoogaming councillors represent the First Nation, which lends to this same idea that the First Nation has greater opportunity to replace these representatives should it be dissatisfied with regard to drinking water quality management or progress thereof ${ }^{20}$.

\section{Drinking Water System Design}

Longlac. The "Town of Longlac Water Treatment System" was constructed in 1983 and has had only minor issues (Salma, 2010). The treatment system is a solids contact type plant, which consists of a conventional two-train filter system (OCWA, 2012a). Treatment includes the use of aluminum sulphate for coagulation and a polymer for flocculation. Chlorine gas is used as the treatment chemical, and Aqua Mag orthophosphate is added for corrosion control.

The plant has a production capacity of $6,050 \mathrm{~m}^{3} / \mathrm{d}$ of treated water. The daily maximum demand for treated water in 2011 was $1,734 \mathrm{~m}^{3} / \mathrm{d}$ (OCWA, 2012b). A OCWA

\footnotetext{
${ }^{20}$ Failures to do so, along with continued stagnation in drinking water quality improvement, may illustrate that the community is a) uninformed about drinking water quality issues, b) satisfied with the majority of other community issues, c) there are no viable alternatives, or d) blaming AANDC and other federal departments for these issues.
} 
interview respondent noted that the Longlac facility needs to run only one of its three pumps, as its capacity is significantly beyond what is needed (personal communication, April 23, 2012). Further, there have been no reports of water shortages for the community (MOE, 2012e). The community has met the maximum $75 \%\left(4,538 \mathrm{~m}^{3} / \mathrm{d}\right)$ operating requirement of total capacity and is designed with sufficient flexibility to service the community (MOE, 2012a; Salma, 2010).

The OCWA interview respondent indicated that the drinking water system is in compliance with all provisions of the Safe Drinking Water Act, 2002, such that "in the last three inspections, there have not been any [sanctions for design], only minor issues here and there - mostly maintenance" (personal communication, April 23, 2012). The MOE inspection report indicates that the system meets $98.3 \%$ (858) of all risk factor requirements (873) (MOE, 2012e). The only underlying issues include a need to implement source water protection measures and to upgrade the automated supervisory control and data acquisition (SCADA) monitoring system.

Ginoogaming First Nation. Ginoogaming First Nation relies on the "Town of Longlac Water Treatment System", which is described above, as well as the Ginoogaming distribution system. Both Salma (2010) and the Ginoogaming interview respondent suggested the design of the distribution system is in compliance with the provisions within the Protocol (personal communication, April 24, 2012).

Comparison of community drinking water system design. Both Longlac and Ginoogaming utilize the same drinking water treatment system; therefore there are no differences in the design of the treatment system. Each of the community's distribution 
systems comply with the design requirements of their respective guidelines/standards (MOE, 2012; Salma, 2010)

\section{Source Water Protection}

Longlac. Longlac does not have a source water protection plan in place (MOE, 2012e). According to the OCWA interview respondent, there is "no specific water source protection as a rule in the north, because [northern communities] are at the top of the watershed" (personal communication, April 23, 2012). The respondent did note, however, that the municipality had conducted risk assessment and identified a number of risks associated with the water system. The MOE report indicates that Longlac is not meeting the source protection requirements within the Safe Drinking Water Act, 2002. The interview respondent articulated that "with shrinking populations and increasing costs to maintain infrastructure, [the community does not] have the money to do it" (personal communication, April 23, 2012).

Ginoogaming First Nation. Ginoogaming First Nation does not have a source water protection plan in place (Salma, 2010). Salma recommended that the community work with the municipality of Greenstone to develop such a plan to comply with the Protocol. The Ginoogaming interview respondent suggested that there is a need to protect the source water from overflowing wastewater during the spring melt, an element allegedly covered by the MTA (personal communication, April 24, 2012).

Comparison of community source water protection. There are no significant differences between Longlac and Ginoogaming with regard to source water protection measures However, Ginoogaming has deferred to Longlac to undertake and fund source 
water protection measures due to the presiding MTA. Neither community is in compliance with the regulations and/or guidelines/protocol to which it is subject.

\section{Operations and Maintenance}

Longlac. The OCWA has a six-year maintenance plan for the Longlac drinking water system (personal communication, April 23, 2012). Budgets for O\&M are developed on an annual basis. The budget for Greenstone's drinking water systems was not disclosed. The OCWA relies on a fixed budget for O\&M, which can be challenging due to fluctuating needs. The most difficult aspect of such budgeting is with regard to obtaining infrastructure funding. As a result of these difficulties, the OCWA does what it can to keep costs down and is sometimes required to cut key components out of the system to effectively operate the system on a fixed budget (personal communication, April 23, 2012). Regardless of these challenges, the plant has performed O\&M in compliance with the Safe Drinking Water Act, 2002 (MOE, 2012e).

Ginoogaming First Nation. Although O\&M activities of the Longlac treatment plant are undertaken by the OCWA, Ginoogaming is responsible for the O\&M of the distribution system. Ginoogaming is currently developing an O\&M manual. The community's WTO indicated that this amount of funding is not sufficient for normal operation of the distribution system (Salma, 2010). Salma suggests that an additional $\$ 42,500$ per annum would be required to meet all O\&M requirements within the Protocol. This amount of recommended funding amounts to $27.8 \%$ of the O\&M funding AANDC presently provides to Ginoogaming for both the drinking water and wastewater systems. Therefore, AANDC has not fully covered the $80 \%$ of total O\&M costs for which it is responsible. Shortcomings in O\&M have led to leaks within the distribution system; 
however, Salma (2010) reports that the First Nation's efforts for undertaking O\&M activities is acceptable and there are no concerns with the WTOs' reliability for O\&M.

Comparison of community operations and maintenance. Both Longlac and Ginoogaming face significant challenges with regard to funding $O \& M$, however, the First Nation is relatively disadvantaged. Specifically, Ginoogaming's O\&M budget has been deemed insufficient for normal operation of the distribution system, whereas the OCWA has been able to meet all Ontario O\&M regulations. This difference is of concern, as leakages in the Ginoogaming distribution system present an opportunity for treated drinking water to become contaminated via seepage through the holes and/or cracks that result in the distribution leaks. Longlac has no such concerns by comparison. ${ }^{21}$

This disparity in funding is the result of how these communities obtain O\&M funds, where Longlac relies on user-fees and Ginoogaming is reliant on AANDC transfers. While the OCWA is provided with static budgets similar to Ginoogaming, the funding appears sufficient provided that the organization is flexible. By contrast Salma suggests that funding is simply insufficient for normal operation. This difference may be reflective of the gap regarding governance actors' approaches to funding, as identified in their theories of change. Specifically, AANDC believes that transfers should be used more efficiently to accommodate for unanticipated needs, whereas the AFN and CoO suggest that greater efficiencies will not be sufficient for producing necessary funds.

\footnotetext{
${ }^{21}$ It is significant to note that the average municipal system has approximately $30 \%$ leakage (EC, 2012). Therefore, it is likely that Longlac has some form of distribution leakage. However, the difference between these communities is the concern expressed by the engineer reviewing the Ginoogaming system and the apparent lack of concern by the the engineer reviewing Longlac's system.
} 


\section{Drinking Water Quality}

Longlac. Drinking water quality is monitored by OCWA's WTOs (personal communication, April 23, 2012). These individuals sample water for bacterial and chemical parameters at a frequency in compliance with the Safe Drinking Water Act, 2002 (MOE, 2012e). Analyses of key parameters are provided in Annex 11. It is evident that drinking water quality measures meet or exceed the provincial standards (MOE, 2012f). The community report illustrates that on three occasions a parameter exceeded the maximum allowable concentrations; however, these issues each pertained to excess turbidity and were remedied the same day by cleaning the system filters.

Ginoogaming First Nation. Drinking water quality prior to reaching Ginoogaming is monitored by the OCWA. Monitoring drinking water quality within Ginoogaming's borders is the responsibility of the First Nation's WTOs, CommunityBased Water Monitors (CBWMs), and HC employed Environmental Health Officers (EHOs). Interestingly, Ginoogaming's WTOs and CBWMs have failed to collect all microbiological test data that is required by the Protocol. A Ginoogaming interview respondent articulated that water monitoring only occurs on a bi-monthly basis, as compared to the recommended weekly measurement, because drinking water quality is assumed to be unlikely to change (personal communication, April 24, 2012).

Specific levels of select chemical and biological parameters of water in the First Nation community are included in Annex 11. These analyses illustrate that water quality in the community occasionally (monthly) fails water quality parameter concentration concentrations related to health, as set out in the GCDWQ. Specifically, chlorine residuals were below standards for $95 \%$ of the samples collected; however, these chlorine 
residuals are consistent with the Safe Drinking Water Act, 2002 (Salma, 2010). Other water quality failures related to aesthetic parameters were also prevalent, such as dichlorophenol and hardness; however, the low magnitude of these non-health related exceedences were not cause for concern (Salma).

Comparison of community drinking water quality. Drinking water quality for these two communities is highly consistent; however, Ginoogaming's monitoring practices were inadequate throughout the distribution system. This difference in monitoring is significant for drinking water quality as contamination could occur throughout the distribution system, particularly with leaks in this system. This difference appears to reflect the gap pertaining to monitoring in the First Nations drinking water quality governance actors' change theories. While the three federal department actors all emphasized monitoring as a primary step in their change theories, the organizations representing First Nations (the AFN and $\mathrm{CoO}$ ) did not appear to include monitoring within its change theory. Ginoogaming's apparent comfort with current drinking water quality, as illustrated by the interview respondents comments that "quality hasn't changed [so monitoring is] not done as often", demonstrates a need to further inform this community of the importance for consistent monitoring. Informing First Nations is consistent with activities emphasized within AANDC, EC, and HC change theories.

There were also several differences in the measures used to evaluate drinking water quality such that the OCWA was not required to measure as many parameters as Ginoogaming. This difference is a reflection of the parameters listed in the GCDWQ and the Safe Drinking Water Act, 2002, which is of concern when comparing drinking water quality of Ginoogaming to Longlac. That is, a higher number and more stringent drinking 
water quality measures may overstate drinking water quality issues in Ginoogaming relative to Longlac. Indeed, such an overstatement occurred with regard to chlorine residuals, such that the First Nation's chlorine residual was below the standards of the Protocol but met the provincial requirements. ${ }^{22}$

\section{Improvement Recommendations}

Longlac. According to the OCWA interview respondent, there have been a variety of system improvements to the Longlac drinking water system and additional improvements are recommended (personal communication, April 23, 2012). Details of incurred improvements are listed in Annex 12. In all, ten system improvements were made between 2010 - 11 totalling $\$ 59,093$. Five pertained to infrastructure investments and equipment replacement, costing $\$ 16,006$ (27.1\%). The remaining five $(50.0 \%)$ improvements related specifically to the O\&M of the system and totalled $\$ 43,087$, representing $72.9 \%$ of recent expenditures.

The OCWA interview respondent indicated that a variety of system improvements will be needed in the foreseeable future, including a basic plant overhaul (personal communication, April 23, 2012). The rationale for such extreme measures is that the system is nearly 30 years old and most components have already surpassed their 20-year life span. The MOE has recommended only that a source water protection plan be developed, which may demonstrate that provincial evaluations overlook long-term needs of drinking water systems (MOE, 2012e).

Ginoogaming First Nation. Salma (2010) made few improvement recommendations for the drinking water system, as non-distribution improvements

${ }^{22}$ The GCDWQ require a minimum chlorine residual of $0.2 \mathrm{mg} / \mathrm{L}$ in $95 \%$ of all monitoring tests. By contrast, the regulations under the Safe Drinking Water Act, 2002 recommends an optimum concentration of $0.2 \mathrm{mg} / \mathrm{L}$ but suggests that residuals can range from $0.05-4.0 \mathrm{mg} / \mathrm{L}$. 
Longlac's responsibility. Those that were suggested can be broken down into current expenditures to meet the Protocol and future expenditures to meet projected serviceability requirements. An overview of immediate recommendations is provided in Annex 13. In total, Salma makes seven recommendations totalling $\$ 84,500$ expenditures for this First Nation to immediately meet current guidelines. Of these recommendations, one (14.3\%) pertains to research and accounts for $\$ 35,000(41.4 \%)$ of the total. One (14.3\%) suggested improvement addresses source water protection. One (14.3\%) immediate recommendation is with regard to infrastructure investments, costing $\$ 9,500(11.2 \%)$. Another (14.3\%) suggested improvement pertains to training and accounts for $11.8 \%$ $(\$ 10,000)$ of total costs. Two $(28.6 \%)$ recommendations are with regard to the O\&M of the water system and account for $27.2 \%(\$ 23,000)$ of estimated costs. Finally, one (14.3\%) recommendation relates to mitigating against contamination crises and accounts for $11.8 \%(\$ 10,000)$ of costs.

Second, future projected costs include the expansion of the MTA, which will require $\$ 305,000$ in capital costs and $\$ 162,000$ in annual O\&M costs. The expansion of the distribution system to accommodate all homes is anticipated to cost $\$ 68,000$. These future costs total $\$ 535,000$ and include an equal number of recommendations for O\&M and water system infrastructure. O\&M account for $30.3 \%(\$ 162,000)$ of these future recommendations, while capital costs pertain to $69.7 \%(\$ 305,000)$. In 2011 Ginoogaming obtained $\$ 251,230$ in additional funding from AANDC for upgrading the distribution and wastewater systems (Ginoogaming, 2012c). Specific funds dedicated to the distribution system were not specified. 
Comparison of community improvement recommendations. Despite the fact that recommended improvements for Ginoogaming apply only to the distribution system, the recommendations for the First Nation are significantly greater in number and cost than those recommended for Longlac. Ginoogaming is missing several important components that are needed to comply with the Protocol including: research, source water protection, infrastructure, training, $0 \& \mathrm{M}$, and emergency response. By contrast, recommendations for the Longlac facilities consist only of upgrades to existing infrastructure and equipment. This difference is significant because it illustrates that even though Ginoogaming is only responsible for making improvements to its distribution system, the First Nation's drinking water quality issues are not specific to one easily repairable component of the change theory. By comparison, recommendations for the OCWA only comprise two such components. Therefore, to improve drinking water quality in Ginoogaming, focus for must be widespread to meet a consistent level of drinking water similar to Longlac over the long term.

There is a disparity in the financial resources available to meet the relevant standards/guidelines for the two communities. Of the funds required for Ginoogaming to comply with the Protocol, $\$ 42,500(50.3 \%)$ are tied to activities assigned to AANDC and/or Ginoogaming funding responsibilities (i.e., infrastructure, O\&M, training). The remaining $\$ 42,000(49.7 \%)$ of recommended improvements is not specifically assigned to a governance actor. For example, although there is a recommendation to research the distribution leakage, it is unclear whether this would be Ginoogaming's or AANDC's responsibility to fund and complete. Without assigning the activity to one actor, it is likely that the activity will not be undertaken. By contrast, Longlac's clear funding 
structure ensures that such activities would be assigned by the municipality and undertaken by either a municipal employee or a OCWA employee.

A notable difference resulting in the recommendation disparity of Longlac and Ginoogaming is in relation to ongoing O\&M activities. Specifically, O\&M activities at Ginoogaming are limited primarily due to funding, whereas the OCWA is able to sufficiently operate and maintain the Longlac facilities. This difference could result in the need for further infrastructure improvements, as Ginoogaming's distribution is more likely to deteriorate without proper O\&M. Indeed, the discussion of the governance actors' change theories demonstrate O\&M funding as a primary issue for long-term sustainability of equipment in First Nations.

\section{Conclusion}

The analyses throughout this chapter illustrate a variety of themes regarding differences in the oversight of drinking water quality for these two analogous communities including significant differences in: financial capacity; human resource capacity; and evaluation. First, it is clear that Ginoogaming has a greater number of deficiencies as a result of funding challenges than does Longlac (OCWA). The primary reasons for this difference are that Longlac is reliant on user fees to fund the water system and that Longlac has been incorporated into the Municipality of Greenstone. The amalgamation has provided the community access to a greater pool of funds than would have otherwise been available. By contrast, Ginoogaming is primarily reliant on AANDC transfers to fund the MTA and the distribution system. Indeed, it has been noted throughout this chapter that the funding provided to Ginoogaming for the community's oversight of the distribution system is insufficient for normal operation and unanticipated expenses. Also noted, this 
difference is a reflection of a gap in the governance actors' change theories such that AANDC indicates that sufficient funding is provided if greater efficiency were utilized, whereas the First Nations groups suggest that current funding is inadequate, even with more efficiencies.

Second, Longlac has access to a number of trained WTOs that are able to effectively operate and maintain the drinking water system, whereas Ginoogaming's WTOs have not obtained training. Indeed, it has been illustrated that there may even be a disincentive for Ginoogaming to ensure WTOs are fully trained. Therefore, Ginoogaming has less human resource capacity than does Longlac. This difference is reflected within the governance actor change theory gap pertaining to emphasis on human resource capacity. Specifically, the AFN and $\mathrm{CoO}$ suggest that this difference is a product of funding issues, whereas AANDC indicates that First Nations need to understand the importance of training and ensure that personnel receives this training using available funding.

Finally, this comparison has illustrated differences in the evaluations of Longlac and Ginoogaming. Specifically, there was alleged disparity in the communities' drinking water quality as a result of different measures, when in fact the water quality was the same. Therefore, differences in evaluation may illustrate that disparity of drinking water quality in First Nations and non-First Nations communities can be overemphasized. However, there is disparity with regard to other comparatives such as training, funding, and O\&M activities, such that Ginoogaming is disadvantaged for providing quality drinking water to its residents relative to Longlac. 


\section{Chapter 5: A Comparison of Communities using Groundwater as their Source of Drinking Water}

The following chapter is the second of three matched-pair case studies. This chapter examines two communities that rely on groundwater as their drinking water source, Dowling Ontario and Sagamok Anishnawbek First Nation (hereafter Sagamok). These communities are similarly located, have similar populations, and use a similar drinking water source. First, Dowling is located approximately 30 kilometers west of Sudbury, while Sagamok is located approximately 90 kilometers west of Sudbury. Second, Dowling is home to 1,690 residents (Statistics Canada, 2012b), while 1,036 residents occupy Sagamok (Statistics Canada, 2012a). Finally, each community supplies its populations with drinking water from two groundwater sources. Therefore these communities are analogous based on each of the three primary determining factors of drinking water quality, as identified in Chapter One.

Despite these similarities, differences are prevalent in the oversight of these communities' drinking water systems stemming from the overall governance structures. Dowling is subject to the two-tiered structure described in chapters one and four. The Protocol for Centralised Drinking Water Systems in First Nations Communities (the Protocol) and the Guidelines for Canadian Drinking Water Quality (GCDWQ) guide Sagamok in all drinking water quality activities. The Protocol stipulates that if the guidelines and/or regulations of a given province (in this case Ontario) are more stringent than the Protocol or GCDWQ, those specific provincial guidelines should direct the First Nation. Therefore, the documents guiding Sagamok are more comprehensive than are those regulating Dowling. By contrast, the Ontario Ministry of the Environment (MOE), 
in conjunction with other provincial ministries, establishes and enforces drinking water standards that Dowling must adhere to under the auspices of the Ontario Safe Drinking Water Act, 2002.

The goal for this chapter is first to explore differences in drinking water quality management at the community level for Dowling and Sagamok. Second, this chapter has been devised to determine whether these differences align with the gaps identified between First Nations governance actors' change theories illustrated in chapter three. These communities are compared based on several facets of drinking water quality management including brief descriptions of each community (including local drinking water governance) is provided, the design of each community's drinking water system, source water protection measures utilized, operations and maintenance (O\&M) practices implemented, drinking water quality measures, recommended drinking water system improvements.

The primary data sources informing this chapter are engineer reports on the risks for drinking water contamination at Dowling (MOE, 2012a) and Sagamok (Paznar, 2011). The Dowling report was submitted to the Ontario Ministry of the Environment (MOE) by an unnamed engineer assessor who was independent of the community. The Sagamok report was created by an engineer contracted by Neegan Burnside as part of the National Assessment of First Nations Water and Wastewater Systems. This engineer was similarly independent of Sagamok Anishnawbek First Nation. Both reports are assumed to have been written without bias. Information within this chapter was also supplemented 
with interviews from Greater Sudbury's Water/Wastewater division and information from Greater Sudbury's and Sagamok's websites ${ }^{23}$.

\section{Community Descriptions}

Dowling. Dowling Ontario was incorporated into the city of Greater Sudbury in 2001 as part of the Ontario government's push to encourage townships cities to amalgamate into larger municipalities (Greater Sudbury, 2012a). The rationale of these groupings was for Ontario communities to pool financial and human resources dedicated to social goods to foster greater flexibility of funds and economies of scale. Other cities and townships incorporated into this municipality include the cities of Sudbury and Valley East as well as the towns of Capreol, Nickel Centre, Rayside-Balfour, and Walden (Canadian Encyclopedia, 2012) ${ }^{24}$. Greater Sudbury has a population of 160,770 (Statistics Canada, 2012c).

Greater Sudbury is governed by an elected mayor and 12 city councillors each of whom represent a particular ward (Greater Sudbury, 2012c). One councillor represents Dowling in addition to four nearby communities. Dowling's drinking water services are under the auspices of Greater Sudbury's Community Infrastructure department (Greater Sudbury, 2012d), which manages water services provided to all of Greater Sudbury through six well systems and two surface water systems (KPMG, 2011). The General Manager of Infrastructure Services oversees the entire department and the Director of Water/Wastewater presides over all aspects of the municipality's water and wastewater

\footnotetext{
${ }^{23}$ Four Sagamok Anishnawbek First Nation public officials were contacted with regard to participating in an interview for this study, each of whom declined. Neegan Burnside was also contacted to provide further comment to the report but indicated that AANDC required the firm to sign a gag order and thus could not participate legally.

${ }^{24}$ Interestingly, Dowling was part of Onaping Falls, as it was incorporated into this township in 1973 (Greater Sudbury, 2012b). Onaping Falls consisted of three communities including Dowling, Onaping, and Levack.
} 
systems. In total, the Water/Wastewater division houses 136 full time equivalents (FTEs), 98 of which are directly involved in the workings of the municipality's drinking water systems (see Annex 14) (Greater Sudbury, 2012e). These individuals contribute to both the Engineering Services division, which undertakes the design and construction of water systems, as well as the Operations division, which "provides for the operation and maintenance of potable water supply and distribution systems" (Greater Sudbury, 2012f). Dowling has access to 13 WTOs that serve Greater Sudbury (Greater Sudbury, 2012e). Six of these operators are certified to a Level IV for both treatment and distribution systems (personal communication, April 7, 2012). Four operators are trained at a Level III certification, two at Level II, and one at Level I. Experience of these operators range from 32 to 3 years with the system (personal communication, April 7, 2012). The municipality also employs an electrician and a service person, who are designated as operators-in-training, as well as an instrument technician that has obtained Level I certification. The MOE (2012a) engineer report indicates that the community has a sufficient number of trained personnel to operate and maintain the water system.

Greater Sudbury has adopted a fully user-pay basis for water services, with variable fees of $\$ 2.02 / \mathrm{m}^{3}$ and annual fix fees of $\$ 29.35$ per year (KPMG, 2011). The municipality has committed to maintain and continually improve upon a quality management system specifically designed to supply its residents with "safe drinking water and environmentally responsible wastewater treatment services that comply with all applicable legislation and regulations" (Greater Sudbury, 2012g).

Sagamok Anishnawbek First Nation. The Chief and 12 Councillors at Sagamok are elected according to the electoral regulations governed by AANDC and as established 
in the Indian Act, 1985 (Sagamok, 2012b). In addition to these elected officials, the community is served by 130 public servants that oversee four major departments (Sagamok, 2012c). One such department, the Office of the Lands, Resources and Infrastructure, houses all staff that contribute to the oversight of drinking water for the First Nation (Sagamok, 2012d). Within the department, five FTEs directly participate in activities regarding various aspects of the drinking water system such as budgeting, procurement, and maintenance related to infrastructure (Sagamok, 2012d). These individuals include the director of the department, a public works clerk, the community infrastructure manager, and two maintenance staff. There are three WTOs serving Sagamok including one primary and two secondary operators (Paznar, 2011) ${ }^{25}$. The primary WTO has achieved Level I certification for both treatment and distribution. This level of certification is adequate for the system's treatment process but not its distribution, as it is a Level II classification. There are two secondary WTOs, designated operators-in-training. The community also has access to an outside WTO trained to a Level I certification for treatment and a Level II certification for distribution to provide advice to the primary and secondary WTOs and to aid in emergency situations (Paznar). While Sagamok employs a sufficient number of WTOs to operate the system, these individuals are not adequately trained to be in compliance with the Protocol.

Sagamok Anishnawbek First Nation relies primarily on AANDC transfers to directly fund the drinking water system. The community is also responsible to fund $20 \%$ of the O\&M activities performed on the system. Sagamok has recently obtained ISO 9001 accreditation for its Quality Management Strategy.

${ }^{25}$ The community has advertised for a WTO trainee in its job opportunities page (Sagamok, 2012e) 
Comparison of community descriptions. There are several differences between Dowling and Sagamaok. First, the community of Dowling has been amalgamated into Greater Sudbury, a population 155.2 times as large as Sagamok. This difference is pertinent to drinking water quality as Dowling has access to a greater financial resource pool. The idea here is that Dowling is likely to achieve greater flexibility of funds and economies of scale than Sagamok, with relation to on drinking water infrastructure, training, and O\&M practices. Therefore, the First Nation is likely providing services less efficiently than is Dowling.

Second, the Dowling drinking water system relies on public funding from user fees, whereas Sagamok is dependent on government transfers from AANDC. This difference presents a host of challenges for Sagamok. For instance, the majority of Paznar's (2011) recommended system improvements require the First Nation to fund activities apart from O\&M. These activities appear to be the responsibility of AANDC; however, this remains ambiguous. Without the onus of any one actor dedicating funds toward these activities, they are likely to go unaddressed. Additionally, O\&M funds are based on yearly cost estimates. Therefore, if the funding formula for a given year does not anticipate needed activities, the community is in danger of significant time gaps without adequate funding for drinking water system infrastructure, and/or O\&M activities. This difference reflects divergence of governance actor change theories such that AANDC expressly indicates that funding provided is sufficient and that First Nations must be more efficient with their use of such funds and the AFN and $\mathrm{CoO}$ suggest that funds are insufficient and the formulas used to calculate such funds are outdated. 
Third, Dowling has access to a greater pool of public servants with regard to drinking water quality management than does Sagamok. In fact, there are more public servants in Greater Sudbury's Water/Wastewater division than there are public officials in all of Sagamok. This difference is significant, as the non-First Nation community has access to a larger number of individuals with expertise regarding the development, operations, and maintenance of drinking water systems. Further, many of Dowling's WTOs are trained adequately for operating the drinking water system, whereas none of Sagamok's WTOs are trained to meet the requirements of the Protocol. Therefore, Dowling has greater overall expertise in drinking water system infrastructure development, treatment, and O\&M. As a result, Dowling is more capable of effectively operating and maintaining a drinking water system efficiently and in accordance with all presiding regulations and/or protocols/guidelines in effect. This difference may also reflect a gap in governance actors' theories of change pertaining to training. That is, although all of the governance actors agree that training First Nations WTOs is a necessary activity, AANDC differs from the others in the belief that current funding is sufficient for such training. ${ }^{26}$

Similarities between the local government of Dowling and Sagamok are also apparent. For instance, both communities are represented by an elected government head and council. This similarity is significant for the governance of drinking water quality because each community is democratically represented in carrying out activities pertaining to drinking water quality. The idea here is that if a community is not satisfied with the management of its drinking water, it is able to replace the governing body. One

\footnotetext{
${ }^{26}$ As noted in chapter 4, this may actually result in a disincentive for the First Nation to train WTOs as the only mechanism for ensuring compliance results in fewer funds, as would an increase in WTO earning potential.
} 
major difference in this regard, however, is that Dowling has only one council representative in a larger 12 ward model for Greater Sudbury, whereas Sagamok is represented by all 12 councillors. Therefore, in theory, Sagamok has a greater opportunity to replace the governing body should it be dissatisfied ${ }^{27}$.

\section{Drinking Water System Design}

Dowling. Treatment for the "Dowling Drinking Water System" occurs at the system's two pump houses, Lionel and Riverside, before it is distributed to the community (Greater Sudbury, $201 \mathrm{la}$ ). The method of treatment is chlorination and ultraviolet irradiation (Greater Sudbury, 2010b). Chemicals used for chlorination include chlorine gas and hydro-fluosilicic acid. Ultra-violet irradiation is used, as it is a requirement of the Safe Drinking Water Act, 2002, for all groundwater sources potentially under the influence of surface water. According to the Dowling interview respondent, the system includes continuous monitoring through the supervisory control and data acquisition (SCADA) system, which is a computerized system equipped with alarms (personal communication, April 7, 2012).

The total capacity of the system for producing treated water is $7,200 \mathrm{~m}^{3} / \mathrm{d}$, which vastly exceeds the community's average daily usage of $521 \mathrm{~m}^{3} / \mathrm{d}$ (Greater Sudbury, 2011 a). Because each pump house has a design capacity of $3,600 \mathrm{~m}^{3} / \mathrm{d}$, the system is not reliant on the operation of both simultaneously. A backup generator is available for the Lionel pump house to ensure that power is constantly on hand. The concrete pressure piping and interlock system will automatically lock out the well before a regulatory

\footnotetext{
${ }^{27}$ Failures to do so, along with continued stagnation in drinking water quality improvement, may illustrate that the community is a) uninformed about drinking water quality issues, b) satisfied with the majority of other community issues, c) there are no viable alternatives, or d) blaming AANDC and other federal departments for these issues.
} 
adverse condition occurs, as determined by the SCADA system. The MOE (2012a) engineer report indicates that the system is $100 \%$ compliant with all system design requirements of the Safe Drinking Water Act, 2002.

Sagamok Anishnawbek First Nation. Treatment at the "Sagamok Water treatment system" occurs at two pump houses, the Halfway pump house and the Toulouse pump house, before it is distributed throughout the system (Paznar, 2011). The treatment method for the system is disinfection through chlorination using sodium hyperchlorite, which is injected directly into the system at each pump house. Sagamok's drinking water system also has functional SCADA system in place (Paznar).

With both pump houses, the Sagamok water treatment system has a design capacity of $2,160 \mathrm{~m}^{3} / \mathrm{d}$, which exceeds the daily amount demanded, $1,091 \mathrm{~m}^{3} / \mathrm{d}$ (Paznar, 2011). Three backup generators are available for the Halfway pump house to ensure that power is consistently available. There have been no reports of water shortages within the community. According to Paznar, the treatment system meets all design requirements of the Protocol.

Comparison of community drinking water system design. Both Dowling and Sagamok utilize direct chlorine injection as the primary treatment method at two pump houses. This type of treatment is in compliance with the presiding regulations and guidelines/protocols. Both drinking water systems are in compliance with all other system design requirements of their respective guidelines/standards (MOE, 2012a; Paznar, 2011). One primary difference between the systems is that Dowling uses ultraviolet irradiation because of its designation as potentially under the direct influence of 
surface water. This difference is due to water source designation and protection and is discussed further in the following section.

\section{Source Water Protection}

Dowling. Dowling is meeting its requirement to the provincial government for identifying source water contamination risks (MOE, 2012a). According to an interview respondent, a report of identified risks and source water protection measures taken is submitted to the MOE annually (personal communication, April 7, 2012). This report includes a test to determine "where contamination of groundwater is likely to occur as a result of surface contamination due to natural hydrogeological features" (Greater Sudbury, 2010a). The test indicated that the groundwater system is at high risk of potential surface water influence; however, it is noted that there are no known specific threats to the Dowling drinking water system. Further, it indicates that there is a wealth of research on the watershed, which results in a low level of uncertainty for contamination.

The Greater Sudbury interview respondent indicated that several other measures are also in place to ensure source water protection at Dowling (personal communication, April 7, 2012). For instance, fences have been built around the Dowling systems two pump houses and the well-heads are monitored in compliance with the Safe Drinking Water Act, 2002. There has also been a Source Protection Committee established for the City of Greater Sudbury. Finally, the community is looking at initiatives to better manage road salt (a known contamination risk) to prevent it from entering the watershed.

Sagamok Anishnawbek First Nation. Sagamok does not have a source water protection plan in place and there is currently no intention to establish such a plan (Paznar, 2011). Paznar has recommended that one be immediately established with the 
incorporation of a plan to build a fence around the pump houses as well as well-head integrity measures. As part of ensuring that the source water protection plan is an accurate reflection of the risk to contamination, a hydrogeology study is also recommended to confirm that the groundwater source is not under the influence of surface water (Paznar). If this study illustrates that the water source is in under the influence, the community will likely require an ultra-violet irradiation system, similar to Dowling's.

Comparison of community source water protection. Dowling has taken measures to protect itself against source water contamination, whereas the Sagamok has not undertaken such activities. There has been no progress toward a source water protection plan for Sagamok. This difference occurs despite that both the Safe Drinking Water Act, 2002 and the Protocol require such a document to be established. This difference between the communities is significant because source water protection is a noted element of quality drinking water. Indeed, several source protection measures that have been taken at Dowling which have not at Sagamok. A primary reason this divergence has persisted is the difference in role clarity for First Nations and non-First Nations communities. That is, on one hand the Municipality of Greater Sudbury is directly responsible overseeing source water protection for the community of Dowling, while the MOE enforces regulations pertaining to source water protection. On the other hand, First Nations communities are responsible for developing source water protection plans and it is EC's role to provide advice on such planning. However, EC does not believe its role in this regard is substantial. Additionally, no federal funding is dedicated directly toward source water protection. Sagamok is also not subject to enforceable 
standards comparable to those of the MOE. The importance of source water protection may be lost in the lack of federal government action, reflecting the gap of responsibility for source water protection articulated in the analysis of governance actors change theories.

A second difference exists between Dowling's and Sagamok's use of hydrogeological studies. Dowling has completed a hydrogeological study and has taken measures (e.g., ultra-violet irradiation) to protect its source water in response to the study. By contrast, Sagamok has continued to treat its source water as groundwater (not under the influence). If the Sagamok water source is more susceptible to contamination than is presently assumed, the lack of a source water protection plan becomes more significant for drinking water quality. This second difference between Dowling and Sagamok is likely a reflection of role ambiguity for the First Nation. That is, Dowling, along with all other Ontario municipalities, was required by the MOE to conduct a hydrogeological study, whereas the First Nations drinking water governance actors have not defined their roles with regard to such research. Although each actor articulates the need for research in their theories of change, only EC's discussion of research explicitly addresses studies for source water protection. The responsibility among the four actors for undertaking and funding such research also remains ambiguous.

\section{Operations and Maintenance}

Dowling. According to the 2011 financial plan, Greater Sudbury allocated $\$ 18.2$ million to O\&M (KPMG, 2011). The budget specific for the Dowling system was not disclosed; however, the overall budget on a per resident basis would allocate 
approximately $\$ 191,317^{28}$ to Dowling ${ }^{29}$. According to the Greater Sudbury interview respondent, the annual operational budget specific to Dowling is relatively static, with maintenance costs fluctuating in accordance with the municipality's O\&M plans and schedules (personal communication, May 3, 2012). As such, the community conducts a host of O\&M activities on a cyclical basis. Specifically, an inspection, cleaning, and report on the Dowling water storage tank is conducted every five years. On a separate five year cycle both pump houses are rehabilitated and cleaned (personal communication, May 3, 2012). All other standard O\&M activities follow the maintenance schedule, which comply with all regulations with the Safe Drinking Water Act, 2002 (MOE, 2012a).

Sagamok Anishnawbek First Nation. The First Nation did not disclose a budget for O\&M; however, AANDC indicates that each year the department transfers $\$ 97,069$ to the community for maintaining water assets (both drinking water and waste water) (Paznar, 2011). Paznar estimates that an additional $\$ 38,000$ of funds are necessary for the O\&M of drinking water assets, totalling $\$ 43,500$ per year, $44.8 \%$ more than AANDC currently allocates. Therefore, even if Sagamok did not contribute its required O\&M funding, AANDC is not providing sufficient funds to operate and maintain the system.

A long-term maintenance management plan has been in place in the community since 2010. However, the system does not have an O\&M manual and the emergency response plan is in need of upgrade (Paznar, 2011). Most activities required by the Protocol are completed by the WTOs apart from line swabbing, water reservoir cleaning,

\footnotetext{
${ }^{28}$ Calculated as follows [(Greater Sudbury budget $/$ Greater Sudbury population $) \times$ Dowling population $]=$ $[(\$ 18,200,000 / 160,770) \times 1,690]=\$ 191,316$.

${ }^{29}$ This estimation is made based on the idea that the system is user funded, as noted in the community description.
} 
and equipment calibration. Despite these shortcomings, Paznar has indicated that maintenance efforts were "acceptable".

Comparison of community operations and maintenance. O\&M funding has impaired Sagamok's drinking water quality relative to Dowling. Dowling has standardized funds which have been noted as sufficient for the operation and maintenance of its water system, whereas funds that have been transferred from AANDC to Sagamok have been insufficient for normal O\&M practices in this community (Paznar, 2011). Greater Sudbury allots significantly more toward Dowling's drinking water system $\mathrm{O} \& \mathrm{M}$, even when controlling for a per resident allocation ${ }^{30}$. As a result of this budgeting difference Dowling is better able to ensure that its residents are served by an effective and operational drinking water system than is Sagamok. This difference is best articulated by the fact that only one of Sagamok's two pump houses is currently in operation due to O\&M funding limitations. Indeed, components of O\&M are missing at Sagamok relative to Dowling such as an O\&M manual. Further, the difference in O\&M between the two communities appears primarily due to funding, as both engineering reports suggest that the level of effort was adequate. Therefore the disparity of O\&M is a reflection of the gap pertaining to O\&M funds presented in the governance actors' change theories, such that AANDC suggests that present funding levels are sufficient if First Nations utilize funds more efficiently and the AFN and $\mathrm{CoO}$ articulate that such funds are inadequate regardless of such efficiencies.

\footnotetext{
${ }^{30}$ Using the funding per resident at Greater Sudbury as a baseline, Sagamok would require approximately $\$ 117,280$ for drinking water system O\&M alone. Calculated as follows [(Greater Sudbury budget / Greater Sudbury population) $x$ Sagamok population $]=[(\$ 18,200,000 / 160,770) \times 1,036]=\$ 117,280$.
} 


\section{Drinking Water Quality}

Dowling. Quality of drinking water is monitored by Greater Sudbury's WTOs, two SCADA Specialists, a Quality Management Systems Officer, a Quality Compliance Officer, two Laboratory Technologists, and three Operations Samplers (Greater Sudbury, 2012e). Together, these individuals sample water for bacterial and chemical parameters of the municipality's water systems at a frequency in compliance with the Safe Drinking Water Act, 2002 (MOE, 2012a). Water quality monitoring results for key parameters measured in 2010 are included in Annex 15. Overall, these analyses indicate that the water system met all Ontario Drinking Water Standards except for sodium. Indeed, no drinking water advisories (DWAs) have recently been in effect for this community (MOE, 2012a). Corrective action was taken for excess sodium levels, rectifying the issue one day after the exceedence was detected. The Dowling interview respondent indicated that the community is taking further action to ensure that sodium does not return to such high levels by monitoring the amount of road salts utilized during winter (personal communication, April 7, 2012). Further, because the sodium exceedence was not considered a health issue, the MOE (2012b) has declared that drinking water quality at Dowling is $100 \%$ compliant with standards of required by the Safe Drinking Water Act, 2002.

Sagamok Anishnawbek First Nation. Drinking water quality for Sagamok is monitored by WTOs, Community Based Water Monitors (CBWM), and HC employed Environmental Health Officers (EHO). Paznar (2011) indicated that most of the monitoring practices recommended by the GCDWQ and the Protocol are adhered to by the CBWMs and WTOs. However, records indicate that neither raw water nor 
distribution samples were monitored for microbiological parameters on an adequate basis (Paznar). WTOs and CBWMs articulated that such samples had been collected, but the relevant data was not properly catalogued. Paznar recommended that the drinking water quality monitoring program for the community be updated to ensure that these microbiological are recorded as per the Protocol's requirements.

Specific analyses of drinking water parameters are included in Annex 15. It is important to note that these results are those from Paznar's (2011) report, as HC's monitoring data was not available for review because the First Nation did not sign the necessary release form. The results illustrate that the system met all but one drinking water quality standards within the GCDWQ and the Protocol. The one exception, water hardness, is not a health-based exceedence. Therefore, the drinking water quality at Sagamok meets all health-based standards. No DWAs have been recently in effect for this First Nation (Paznar, 2011).

Comparison of community drinking water quality. A significant difference between Dowling and Sagamok exists with regard to drinking water quality monitoring (specifically record keeping). If one or more bacterial measures were missed and unaccounted for in Sagamok's records, the drinking water supply could be contaminated for a prolonged period of time. As a result, community members could consume microbiologically contaminated water, which is the greatest health risk of public water sources (Hrudey et al., 2002). This difference between the two communities is likely the result of the regulations gap, such that Dowling's measurement and recordkeeping practices are legally enforced by the MOE and Sagamok is only guided by the Protocol. The difference of monitoring practices also reflects a gap in the governance actors' 
change theories, such that the AFN and $\mathrm{CoO}$ was the only actor to not include monitoring as a specific step in the change theory. Therefore, it is reasonable to suggest that raising awareness of the importance of monitoring may be necessary, as indicated by the activities within AANDC's, EC's, and HC's change theories.

Despite the difference in drinking water quality monitoring, both communities met the guideline/standard requirements for all but one parameter measured. Neither of these exceedances was considered a health threat. Therefore, neither Sagamok nor Dowling illustrated current drinking water quality issues of great concern.

\section{Improvement Recommendations}

Dowling. According to the most recent annual water report, and corroborated by an interview respondent, the treatment systems do not currently need improvements apart from regularly scheduled maintenance (Greater Sudbury, 2011; personal communication, April 7, 2012). However, several expenses beyond normal O\&M costs have been incurred since 2004 and are presented in Annex 16. In total, the Dowling drinking water system has required three improvements beyond standard maintenance practices, totalling more than $\$ 115,000$. Two $(66.7 \%)$ of these upgrades pertained to infrastructure investments and accounted for $\$ 60,000$ or $52.2 \%$ of total costs incurred. The remaining (33.3\%) of system improvements addressed cyclical O\&M practices, and represented $47.8 \%(\$ 55,000)$ of the costs reported.

Sagamok Anishnawbek First Nation. Paznar (2011) has recommended a variety of drinking water system improvements for Sagamok. These recommendations are divided into those required for immediate compliance with the Protocol and for future serviceability. Immediately needed costs are presented in Annex 17. In total, Paznar 
(2011) illustrates 14 recommendations totalling $\$ 431,500$, which are needed immediately to bring the community in full compliance with the Protocol. One (7.1\%) recommendation is with specific regard to research and accounts for $12.8 \%(\$ 55,000)$ of these costs. Two (14.3\%) suggestions pertain to source water protection and also account for $12.8 \%(\$ 55,000)$ of immediate expenditures. Infrastructure costs account for three (21.4\%) of the recommendations, totalling $\$ 183,000(42.4 \%)$. Both training and O\&M activities require two (14.3\% each) improvements totalling $\$ 20,000(4.6 \%)$ and $\$ 40,000$ $(9.3 \%)$ respectively. Three $(21.4 \%)$ suggestions were made with regard to water quality monitoring improvements and account for $4.3 \%(\$ 18,500)$ of the total immediate expenditures needed. Finally, one (7.1\%) improvement recommendation pertains to mitigating contamination when it occurs and is estimated to cost $\$ 10,000(2.3 \%)$.

Paznar's (2011) five recommendations for future serviceability total $\$ 4,161,700$. Four $(80.0 \%)$ of these suggestions are with regard to infrastructure development and total $\$ 3,736,000$, or $89.8 \%$ of the future total costs. The remaining $(20.0 \%)$ future recommendation pertains to $O \& M$ and accounts for $10.2 \%(\$ 425,700)$ of these expenditures.

Comparison of community improvement recommendations. There is a significant difference in the improvement recommendations for Dowling's and Sagamok's drinking water systems, which are due to a variety of differences in drinking water quality financing. First, there is a disparity in the financial resources available to service Sagamok's and Dowling's drinking water systems. These immediate costs required for Sagamok to meet requirement of the Protocol total $\$ 431,500$. AANDC is responsible for funding infrastructure investments, training, and $80 \%$ of O\&M activities, 
which account for $56.3 \%(\$ 242,935)$ of recommended costs. Therefore, the community is reliant on the federal department to disseminate these funds. Significantly, the responsibility for funding the remaining $43.7 \%(\$ 188,565)$ of these recommendations is not explicitly assigned to any one governance actor within the protocols or guidelines currently available. For instance, although source water protection is the responsibility of the First Nation and EC is to provide advice on this activity, no funding is directly linked to it. Therefore, it is unclear as to whether the First Nation is supposed to direct its own resources to this activity or if another governance actor will provide funds. If the former is the case for these remaining recommendations, the First Nation will not have the capacity to finance them. By contrast, Dowling has no immediate recommendations, which is partially due to the community's ability to fund all improvements needed as they arise. Dowling has the capacity to fund these activities through its use of a user fees to fully operate and maintain the system. Further, the community also has access to Greater Sudbury's pooled resources to fund major projects. Therefore, Sagamok is disadvantaged, relative to Dowling, with regard to having the ability to fund a drinking water system capable of providing quality drinking water to its community members.

There is also disparity between Dowling and Sagamok with regard to role ambiguity and enforcing required system improvements. For instance, on one hand Dowling was required in to conduct a hydrogeological study in 2002 to determine if the system was under the direct influence of surface water. Following the assessment, the community was required through $\mathrm{MOE}$ regulation to implement ultra-violet irradiation as a precautionary measure, which it complied with soon afterward. On the other hand, Sagamok has been recommended to implement a hydrogeological study to determine if it 
requires ultra-violet irradiation among other mechanisms. To date, this study has not taken place despite it being a requirement of the Safe Drinking Water Act, 2002, and therefore a part of the Protocol. Because the role for funding this research has not been explicitly designated to one aspect (source water protection, system desing, or O\&M) and no regulations are in place to enforce it, Sagamok will continue to operate the system without fully understanding its water source. Therefore, Sagamok will continue to be at risk for serving its residents poor drinking water quality.

The immediate suggested improvements for the Sagamok also span across the majority of steps listed in the change theories discussed in the third chapter including research, source water protection, infrastructure investment, operator training, $O \& M$, and water quality monitoring. This is in contrast to Dowling, which has no needed immediate improvements. This difference is significant because it illustrates that Sagamok's issues pertaining to drinking water quality are not specific to one easily repairable component of the change theory. Focus for improvement must be widespread if the First Nation is to have similar capacity as Dowling for producing quality drinking water.

\section{Conclusion}

The analyses throughout this chapter illustrate a variety of themes regarding differences in the oversight of drinking water quality for these two analogous communities including significant differences in: governance role clarity; financial capacity; human resource capacity; and regulation. First, ambiguity in role definition, particularly with regard to financial matters not directly related to infrastructure and/or O\&M costs, has caused significant portions of Sagamok's drinking water system to go unfunded and unaddressed. By contrast, Greater Sudbury (Dowling) and the MOE have clearly defined 
roles, which reinforce the undertaking of their respective responsibilities for ensuring quality drinking water for Dowling residents. This difference was an explicitly noted foundational assumption of all four First Nations drinking water quality governance actors' change theories. Indeed, ambiguity with regard to financial allocation was of particular importance to the AFN and $\mathrm{CoO}$, whereas AANDC maintains that actual level of funding necessary for First Nations drinking water is unclear. Further differences with regard to role ambiguity are also articulated in Sagamok's source water protection and drinking water quality monitoring.

Second, due to the amalgamation into Greater Sudbury as well as the community's full user-pay system, Dowling has access to funding sufficient for covering standard O\&M procedures as well as any unexpected circumstances that may arise. Sagamok, however, has access only to limited and insufficient (according to Paznar, 2011) funds that are tied to specific activities (i.e., planned infrastructure, budgeted O\&M activities). Therefore, Sagamok does not have financial capacity to cover expected, let alone unexpected, drinking water system expenses. Again, this difference is a reflection on the disagreement with regard to the importance of infrastructure and O\&M funding. Specifically, the First Nations groups suggest that the primary issue is funding, whereas AANDC indicates that adequately trained personnel would be able to operate and maintain these water systems more efficiently, thus without needing additional federal funding. EC and $\mathrm{HC}$ suggest that both increased funding and training are necessary. Third and related, there was a significant difference in the number of trained personnel available to operate and maintain each community's drinking water system. Sagamok is lacking the human resource capacity relative to Dowling for ensuring that its 
residents are supplied with quality drinking water. This difference is reflected in the gaps pertaining to emphasis on human resource capacity. Specifically, the AFN and CoO suggest that this difference is a product of funding issues, whereas AANDC indicates that First Nations need to understand the importance of training and ensure that personnel receives this training using available funding. Again, EC and $\mathrm{HC}$ suggest that both understanding the importance of training and funding that training are issues that need to be addressed.

Finally, these two communities illustrate the regulations gap such that Dowling is required to adhere to regulations for drinking water quality while Sagamok is directed by more stringent guidelines. The difference is prevalent because Dowling is subject to legal enforcement of these regulations, whereas there is no recourse for Sagamok's if it chooses to not follow the Protocol. Interestingly, this difference is related to each of the above discrepancies between Dowling and Sagamok, as roles, financial capacity, and human resource capacity are all elements of the regulations presiding over Dowling. As a result, there is less ambiguity over the roles, responsibilities, and consequences of noncompliance. Further, this distinction between Sagamok and Dowling is a reflection of the relative importance of regulation with regard to each of the First Nations drinking water quality governance actors' theories of change. Indeed, there is considerable disagreement between the four actors regarding when regulations should be implemented. Specifically, AANDC suggests that regulation is immediately needed to foster efficiency and ensure that the protocol is adhered to. By contrast, each of the other three governance actors indicate that the regulation gap should be addressed only after First Nations have the capacity to address them. 
Although these differences between Dowling and Sagamok have not culminated in significant disparity with regard drinking water quality, the First Nation is at a greater risk for drinking water quality issues. This conclusion becomes more concerning when one considers that Sagamok's emergency response plan is in need of upgrade, a task regularly completed at Dowling. Further, the difference pertaining to risk for drinking water quality issues in communities relying on groundwater source has been demonstrated as a reflection of the gaps within the First Nations drinking water quality governance actors' theories of change. 


\section{Chapter 6: A Comparison of Communities using Surface Water as their Source of Drinking Water}

The following chapter is the third of the three matched-pair case studies. This chapter compares two communities which rely on surface water as their source for drinking water, Red Lake Ontario and Pikangikum First Nation. These communities are analogous in that they are similarly located, have a population size, and use a similar drinking water source. First, Red Lake is located approximately 200 kilometres north of Kenora Ontario and Pikangikum is located approximately 95 kilometres north of Red Lake (Red Lake, 2012a). Second, there are approximately 1,274 residents in Red Lake (Statistics Canada, 2012d) and 2,100 residents in Pikangikum (Statistics Canada, 2012e). Third, both communities utilize surface water as the sole source of drinking water, drawing from Red Lake and Pikangikum Lake respectively.

Despite similarities in these three characteristics, the two communities have a number of differences throughout the oversight their respective drinking water systems, which stem from broad divergence in governance structure. Red Lake is subject to the two-tiered structure described in chapters one, four, and five and is regulated by the Safe Drinking Water Act, 2002. By contrast, Pikangikum First Nation's drinking water is governed by four independent actors including the Pikangikum Chief and Council, Aboriginal and Northern Development Canada (AANDC), Health Canada (HC), and Environment Canada (EC). The Protocol for Centralised Drinking Water Systems in First Nations Communities (the Protocol) and the Guidelines for Canadian Drinking Water Quality (GCDWQ) guide Pikangikum in all drinking water quality activities. The Protocol stipulates that if the guidelines and/or regulations of a given province are more 
stringent than the Protocol or GCDWQ, those specific provincial guidelines should direct the First Nation. Therefore, the documents guiding Pikangikum are more comprehensive than are those which regulate Red Lake.

The aim of the chapter is twofold. First, this chapter is devised to explore the differences in community-level water quality management of two analogous communities relying on surface water as their drinking water source. Second, the chapter has been devised to examine whether differences identified reflect the gaps illustrated between the First Nations drinking water quality governance actors' theories of change. In undertaking these goals, the communities are compared across the same elements of drinking water quality management including: brief community descriptions, source water protection measures, drinking water system design, operations and maintenance $(\mathrm{O} \& M)$ practices, drinking water quality measures, and recommended system improvements.

Data informing this chapter are primarily drawn from engineer reports on each community's risk for drinking water contamination throughout the water systems at Red Lake (MOE, 2012c) and Pikangikum (Mayberry, 2011). The Red Lake report was submitted by an unnamed independent engineer to the MOE as part of provincial inspection requirements set out within the Safe Drinking Water Act, 2002. The Pikangikum report was submitted to Neegan Burnside, as part of the National Assessment of First Nations Water and Wastewater Systems, by a contracted engineer independent of the community. Other information from was drawn from community websites to 
supplement this data. Interviews with key officials were attempted for both communities; however, they did not come to fruition ${ }^{31,32}$.

\section{Community Descriptions}

Red Lake. In 1998, the community of Red Lake was amalgamated into the Municipality of Red Lake along with six other townships including Balmertown, Cochenour, Madsen, McKenzie Island, and Starratt-Olsen (Red Lake, 2012a). Such rural integration was encouraged by the Ontario government throughout the mid-1990s and early 2000 s to enable rural communities' access to a greater pool of resources for social goods. The resulting Municipality of Red Lake is home to 4,366 residents (Statistics Canada 2012c).

The City of Red Lake is governed by an elected Mayor and six elected city councillors, one of which represents the community of Red Lake (Red Lake, 2012b). Several municipal employees are involved in the governance of drinking water for the community including the Chief Administrative Officer, Operation Supervisor, and Operations Superintendent (Red Lake 2012c). These individuals are responsible for the infrastructure aspects of the system. O\&M activities are outsourced to a private firm, Northern Waterworks Incorporated.

Northern Waterworks is managed by a Chief Executive Officer, a Chief Operating Officer, and a Chief Administrative Officer, each of whom is a trained to a minimum of level IIl certification for treatment and distribution (Northern Waterworks, 2012c).

${ }^{31}$ Several individuals at Pikangikum directed the author to interview one official in the band administration. This individual participated in an interview but later declined to sign the consent form allowing the author to use any data obtained.

${ }^{32}$ Red Lake officials directed the author to contact the organization in charge of the community's water system's operations and maintenance, Northern Waterworks. A high-level administrator with the organization spoke with the author on several occasions and agreed to participate in an interview; however, this individual was unable to schedule an interview during the two months he and the author were in contact. 
Northern Waterworks employs 10 WTOs that work in the Municipality of Red Lake. Each of these WTOs meets or exceeds the certification requirements of the Red Lake community drinking water system (MOE, 2012c). The firm also has a large pool of 22 WTOs that work outside of Red Lake. The specific certification levels and years experience of these individuals were not disclosed. The MOE has indicated that there are a sufficient number of trained personnel to operate and maintain the drinking water system in compliance with the Safe Drinking Water Act, 2002.

A breakdown of the specific financial resources allocated for the Red Lake community's drinking water system was not made available. The Municipality of Red Lake relies primarily on variable user fees of $\$ 1.12 / \mathrm{m}^{3}$ for funding the drinking water system (Red Lake, 2012 d). The municipality also receives $\$ 25,000$ per year from the Ontario Small Waterworks Assistance Program, which is a program that provides "entitlement operating funding to municipalities with public drinking water systems serving between 1,001 and 5,000 residents" [Ministry of Infrastructure (MOI), 2012a]. The program also provided an additional $\$ 717,496$ for the municipality's water infrastructure renewal (MOI, 2012b).

Pikangikum First Nation. Pikangikum, a community accessible by plane and boat or ice roads, is governed by a Chief, Deputy Chief, and nine Councillors [Whitefeather Forest Initiative (WFI), 2012]. These individuals were elected through custom election rules, as enabled in the Indian Act, 1985 (WFI). While the Chief is the head of the community's administration, the community notes that "consensus-based participatory decision-making is highly prized" (WFI). Management of the community's drinking water falls under the Community Infrastructure department of the band 
administration, which is headed by the Public Works director [Independent First Nations Alliance (IFNA), 2012]. Pikangikum also employs three WTOs, two of which have no training certification (Mayberry, 2010). The primary WTO has achieved Level I certification for treatment only, which is below the plant's designation as a Level II facility. Mayberry and the NWHU (2006) suggest that the WTOs are highly knowledgeable about the Pikangikum system and the requirements its operation. Although Pikangikum employs a sufficient number of WTOs to operate and maintain the drinking water system, these individuals are not adequately trained to fulfill requirements of the Protocol (Mayberry) ${ }^{33}$.

Pikangikum relies primarily on AANDC contribution agreement transfers to fund infrastructure investments and O\&M costs of its drinking water treatment facility. AANDC had reportedly invested $\$ 3,998,996$ in water and wastewater projects at Pikangikum from 2006 to 2010 (INAC, 2010). These funds were used for both capital investments $(\$ 1,572,265)$ and O\&M $(\$ 2,426,731)$. In 2007 , the department promised $\$ 9.7$ million in new funds to establish water servicing directly to the community's homes (AANDC, 2007). These funds have yet to be disseminated. Pikangikum is responsible for funding $20 \%$ of its drinking water O\&M activities.

Comparison of community descriptions. Several differences between Red Lake's and Pikangikum's management of their drinking water systems are prevalent beginning with access to pooled resources. Because Red Lake has been incorporated into

\footnotetext{
${ }^{33}$ In 2001 the Department of Indian Affairs and Northern Development (later Indian and Northern Affairs Canada and subsequently AANDC) attempted to unilaterally appoint a third party to manage Pikangikum's finances without precedent (Magnet, 2001). Indeed, the department failed to follow standardized procedures for imposing third party management (Blatchford, 2010). The court ruled that the department acted illegally and quashed the decision (Fox, 2002). In fact, the ruling judge indicated that the department was seeking to gain inside information on the First Nation's forestry financial arrangements with Ontario.
} 
a municipality larger (2.1 times) than Pikangikum, the community has access to a larger base of financial and human resources. The idea is that Red Lake has more flexibility and is more capable than is Pikangikum to achieve economies of scale for infrastructure, training, and O\&M spending with regard to its drinking water system. As a result, Red Lake is better positioned to efficiently provide drinking water to its residents than Pikangikum.

Second, Red Lake relies primarily on user-fees for operating its drinking water system, with assistance from the MOI, whereas Pikangikum relies primarily on AANDC for such funds. This difference provides significant challenges for Pikangikum relative to Red Lake, as the majority of recommended system improvements at Pikangikum require AANDC funds (Mayberry, 2010). Relying on an external source for the majority of the system's funding is problematic the activities will likely go uncompleted if funds are not sufficiently allocated. Further, such funds are budgeted on yearly cost estimates, which have been criticized for underestimating true costs by the AFN and CoO. This difference reflects the divergence of governance actor change theories, such that AANDC believes funds provided are sufficient if greater efficiencies are found whereas the AFN and CoO suggest that funds are insufficient regardless of any additional efficiencies that can be found.

Third, the Red Lake has outsourced O\&M activities to Northern Waterworks, whereas Pikangikum continues to oversee these aspects of its system. This difference is pertinent to each community's drinking water quality, as Red Lake has contracted experts in the field of drinking water to oversee its system, while Pikangikum continues to utilize undertrained personnel to operate its own system. Further, Northern Waterworks employs 
more WTOs with high levels of training and certification than does Pikangikum.

Therefore, Red Lake has greater overall expertise than Pikangikum in its drinking water system and is more capable of effectively operating the system in accordance with all regulations and/or protocols/guidelines in effect. As illustrated in chapters four and five, the difference in training may reflect the divergence between governance actor's change theories, such that the AFN and $\mathrm{CoO}$ suggests further funding is required while AANDC maintains that current funding for training is sufficient.

A fourth pertinent difference between Red Lake and Pikangikum is that the former is more easily accessible, as it has year-round road access. This difference is significant to the community's respective drinking water systems, as added barriers to transportation increase the costs for delivering a variety of needed supplies such as building materials, equipment, and treatment chemicals. As such, Red Lake has greater ease of access than Pikangikum to building materials and new equipment for infrastructure as well as needed commodities for O\&M.

It is also worth noting the similarities between Red Lake and Pikangikum with regard to local government. Specifically, both communities are represented by an elected head and council, which is pertinent for the governance of drinking water quality as both communities are democratically represented in carrying out activities that pertain to drinking water. Ideally, each community is able to replace its current governing body at the end of the electoral cycle should it be dissatisfied with the local management of drinking water quality. Interestingly, Pikangikum utilizes a shorter electoral cycle (two years) than does Red Lake (four years). Further, all Pikangikum councillors represent the community, whereas only one councillor represents the community of Red Lake. 
Therefore, in theory Pikangikum has greater opportunity to replace its local government should it be dissatisfied with the management of drinking water quality ${ }^{34}$.

\section{Drinking Water System Design}

Red Lake. The "Red Lake Drinking Water System" is a "conventional, chemically assisted filtration plant with two treatment trains providing coagulation, flocculation, filtration, and chlorine disinfection" (Northern Waterworks, 2010). Filtration chemicals include polyaluminum chloride and a catatonic polymer for coagulation and flocculation. Once water is filtered through the system it is treated using chlorine gas and sodium carbonate.

The water system was in compliance with nearly all specifications at the time of MOE inspection (MOE, 2012c). The designed capacity of the drinking water system is $6,048 \mathrm{~m}^{3} / \mathrm{d}$, which exceeds the amount community's daily demand of $2,465 \mathrm{~m}^{3} / \mathrm{d}$ (Northern Waterworks, 2011). The system utilizes less than $75 \%$ of the system capacity, as required by the Safe Drinking Water Act, 2002. Valid permits and licenses were obtained and on display for the treatment facility. The only issue identified was that the chemical metering pumps installed for the system had a rated capacity lower than that required by the treatment plant's permit (MOE, 2012c). However, the capacity of these pumps was in the range of the flow rates at which the plant was operating. A simple form amending the permit needed to be filed to rectify this non-compliance.

Pikangikum First Nation. The "Pikangikum Water Treatment System" consists of a conventional two-train chemically assisted filtration package that also provides

\footnotetext{
${ }^{34}$ Failures to do so, along with continued stagnation in drinking water quality improvement, may illustrate that the community is a) uninformed about drinking water quality issues, b) satisfied with the majority of other community issues, c) there are no viable alternatives, or d) blaming AANDC and other federal departments for these issues.
} 
flocculation and sedimentation (Mayberry, 2010). Filtration chemicals include aluminum chloride and polymer blends as coagulants. A granular media technology is used to filter the water directly at the water treatment plant. Once water is filtered it is treated with sodium hypochlorite, which is injected into the water prior to its collection in a reinforced concrete reservoir. Interestingly, there is no direct distribution system to the community's homes. Instead, there are eight watering points (in-ground taps) available to community members throughout the reserve land, which is not large enough to receive a distribution classification (and thus no standards apply). Residents collect drinking water from these taps using five-gallon pails (Harden \& Levalliant, 2008). It has been noted that about half of the community draws drinking water directly from Pikangikum Lake, as they have difficulty accessing the watering points (Swain, Louttit, \& Hrudey, 2006).

Mayberry (2010) has indicated that the overall design of the treatment plant is compliance with the Protocol. The designed capacity of the water treatment system is $1,036 \mathrm{~m}^{3} / \mathrm{d}$; however, the treatment plant is currently able to produce only $345 \mathrm{~m}^{3} / \mathrm{d}$ due to faulty flocculators in both of the trains of the filtration process (Mayberry, 2010). These issues are due to O\&M lapses, but have resulted in the requirement for new infrastructure. Pikangikum's maximum daily demand for treated drinking water 259 $\mathrm{m}^{3} / \mathrm{d}$. Interestingly, Mayberry estimates that if an adequate distribution system were in place maximum demand would be $1,290 \mathrm{~m}^{3} / \mathrm{d}$. Therefore, Mayberry indicates that while the system is designed to meet current daily demand, the community's demand is grossly underestimated. She suggests that strictly following the assessment measures illustrate that the community is in compliance with the Protocol, but provides the caveat that the system is not adequately designed to ensure that the First Nation is served with the same 
quality of drinking water as non-First Nations communities. There are no current plans in place to expand the treatment or distribution systems.

Comparison of community drinking water system design. Both Red Lake and Pikangikum use conventional drinking water treatment systems involving two-train chemically assisted treatment filtration including flocculation and coagulation. Both systems utilize chlorine treatment where Red Lake uses chlorine gas and Pikangikum uses sodium hypochlorite. Both of these treatment chemicals are appropriate for a surface water sources and are commonly used. Furthermore, both communities' treatment systems are designed in compliance with the Safe Drinking Water Act, 2002 and the Protocol respectively.

Despite the on-paper compliances, there are significant differences with Red Lake's and Pikangikum's distribution systems. Specifically, Red Lake has a standard distribution system for the size of the community, whereas Pikangikum does not have a classifiable distribution system. This difference is of great concern for drinking water quality, as many Pikangikum residents use raw water in lieu of a proper distribution system. This difference is an oversight due to a variety of factors including a gap in regulations presiding over distribution systems and a lack of funding to support distribution infrastructure. Indeed, this difference is a reflection of gaps within the First Nations drinking water governance actors' change theories pertaining to planning and infrastructure funding, where AANDC funds infrastructure based on needs, as identified through non-compliance with the Protocol. Because Pikangikum has not breached the Protocol, there are no justifications for additional funding. 
As a consequence of the lacking distribution system, the designed capacity for the Pikangikum's drinking water system is not sufficient to meet normal needs of a community its size. By contrast, Red Lake has the capacity for meeting its communities treated drinking water demands. This difference is a reflection of gaps with respect to planning and funding within the First Nations governance actors' theories of change, as described above. As a result, the lacking distribution system is overlooked. Thus, drinking water demand remains low in Pikangikum, which further dispels any need for increased capacity. More focused planning and infrastructure spending using expert advice, as per the AFN and CoO's change theory, would lead to the identification and subsequent address of these issues.

\section{Water Source Protection}

Red Lake. Red Lake does not have a source water protection plan; however, the MOE inspection (2012c) indicates that this is not a requirement for community as it is near the top of the watershed. The municipality completed an assessment of the risks to its water source in 2006 , which indicated that there are no notable threats to the source water (MOE, 2012c).

Pikangikum First Nation. Pikangikum does not currently have a water source protection plan (Mayberry, 2010). Such a plan is required by the Protocol. Mayberry noted that there is only a minor risk of source water contamination, as the community is at the top of the watershed.

Comparison of community source water protection. Neither Pikangikum nor Red Lake has a source water protection plan currently in place. Both engineer reports suggest that this is not a significant concern, as there is low risk for source water 
contamination. There does not appear to be a significant gap between the communities with regard to source water protection.

\section{Operations and Maintenance}

Red Lake. Budget details for normal O\&M practices were not available for review, as this was a private corporation; however, the MOE $(2012 \mathrm{c})$ has indicated that Northern Waterworks operates the plant sufficiently, complying with all appropriate licenses and permits pertaining to the Safe Drinking Water Act, 2002. All appropriate contingency plans and operations manuals for the water system (and subsystems therein) were readily available for use by operators. The municipality has several specific programs in place for scheduled O\&M of the system including rehabilitation and replacement of piping, pipe flushing and cleaning, testing and exercising system valves, and monitoring flow rates and water pressure. Red Lake had only one major issue with its O\&M practices according to the MOE, such that there was not routine cleaning, inspection, and maintenance of the community's water tower.

Pikangikum First Nation. Budget details for the operation and maintenance of Pikangikum's drinking water system were not made available; however, the 2006-10 AANDC water investment report indicates that the First Nation received $\$ 2,426,731$ for O\&M of their water and wastewater facilities, an average of $\$ 485,346$ per year (INAC, 2010a). According to estimates provided by Mayberry, the O\&M budget does not "appear to be adequate for normal operation and maintenance of this system". Interestingly, Mayberry estimates that if a distribution system were established and a capacity was expanded to meet community needs, O\&M would require $\$ 441,000$ per year for the drinking water system alone (read: not including wastewater O\&M). The First 
Nation does not have a maintenance management plan in place (Mayberry, 2010). An AANDC approved O\&M manual exists but is not being utilized. Mayberry suggests that none of the following required maintenance activities are conducted: line flushing, hydrant flushing, fire pump testing, and equipment calibration. The lack of O\&M practices has resulted in inoperable flocculators and lift pumps among other deficiencies. Mayberry indicates Pikangikum fails to meet the standards within the Protocol. Indeed, he further states that "the level of effort put into maintenance is substandard". Therefore, O\&M failures are partially due to funding and partially due to effort.

Comparison of community operations and maintenance. Differences between Red Lake and Pikangikum with regard to funds available for drinking water system O\&M practices have resulted in disparity regarding drinking water quality. Red Lake has allocated funds for these activities primarily from user-fees, which are supplemented by MOI contributions and pooled municipal resources. By contrast, Pikangikum is primarily reliant on AANDC for funding O\&M activities. Mayberry (2010) has articulated that the amount of funding allocated to these practices has been insufficient for normal operation of the system. It is not clear whether the First Nation has met its $20 \%$ funding requirement or if AANDC is responsible for the underfunded O\&M. However, the lack of funding has contributed to a treatment system with reduced daily capacity. This issue is a prime example of the funding dispute identified as a gap in the First Nations drinking water quality governance actors' theories of change. That is, the AFN and CoO suggested that AANDC has underfunded O\&M practices, while AANDC claims that First Nations do not allocate funds provided as efficiently as required. 
A second difference between Red Lake and Pikangikum is with regard to the level of effort put into O\&M practices. Specifically, Northern Water works was lauded by the engineer report (2012c) for exceeding nearly all O\&M requirements, whereas Mayberry (2010) suggested that Pikangikum's efforts were subpar. Pikangikum's efforts are of concern, as limited efforts may exacerbate Pikangikum's relative disparity in drinking water quality. It is difficult to ascertain why there is lower effort in Pikangikum than Red Lake, but several possibilities are apparent. For instance, there is more WTO involvement in the development of budgets at Red Lake than Pikangikum, which could result in the latter's WTOs perceiving their work as undervalued. The inadequate O\&M budget allocations similarly devalue the importance of such activities. Finally, the complete lack of a distribution system is likely disheartening, particularly for those individuals that are most knowledgeable about proper drinking water systems. These challenges may reflect the gaps within the governance actors' change theories regarding engagement. Specifically, a prolonged lack of engagement with employees responsible for operating the drinking water system may result in discouragement and subpar effort, which is analogous to the idea that a lack of First Nations engagement will result in prolonged drinking water quality issues for these groups.

\section{Drinking Water Quality}

Red Lake. Red Lake's drinking water quality is monitored by the WTOs at Northern Waterworks (MOE, 2012c). According to the MOE, all organic and inorganic water quality monitoring requirements exceeded provincially legislated monitoring practices. Additional continuous water monitoring occurs through the supervisory control 
and data acquisition (SCADA) system, which enables computerized shutdown of equipment in the event of emergencies or system malfunction.

Specific measures drinking water quality measures are detailed in Annex 18. Only three issues occurred throughout 2011 , including one instance of non-compliance for nitrate/nitrite sample analysis (Northern Waterworks, 2011). This non-compliance issue was simply a result of the WTOs taking the sample five days later than prescribed, rather than an exceedence of this parameter. The second issue was a result of chlorine treatment negatively interacting with excess sedimentation, which caused elevated trihalomethane levels. Corrective action was taken to reduce chlorine treatment and increase coagulation procedures, which resolved the problem within 10 days. Finally, there were four lead exceedances due to aging plumbing, which will require the replacement of piping infrastructure. However, the MOE (2012c) has indicated that there are no concerns regarding health-related parameters of drinking water and that the water system is in compliance with $100 \%$ of water quality regulations.

Pikangikum First Nation. Pikangikum's drinking water quality monitoring is the responsibility of the community's WTOs and Community-Based Water Monitors (BWMs) as well as EC employed Environmental Health Officers (EHOs) (Mayberry, 2010). The Northwestern Health Unit (NWHU, 2006) in Ontario indicated that "EHOs visits were infrequent [and that] water sampling, chemical testing, inspection and education usually provided by EHOs were therefore insufficient to ensure safe water for the community". Despite these challenges, water quality monitoring by WTOs within the treatment plant is in compliance with monitoring requirements of the Protocol (Mayberry). Water is also continuously monitored by a SCADA system; however, this 
equipment is not calibrated on a regular basis (Mayberry). Overall, Mayberry indicates that monitoring practices are appropriate, with a few adjustments to equipment calibration and raw water monitoring.

Specific analyses of all drinking water parameters were not made available for the Neegan Burnside assessment, as $\mathrm{HC}$ failed to sign the appropriate release (Mayberry, 2010). The First Nation did, however, disclose results regarding turbidity and chlorine residuals. These analyses reveal that treated water exceeds the GCDWQ for turbidity $93 \%$ of the time and that chlorine residuals are below the GCDWQ standards $7 \%$ of the time. Mayberry indicated that these failures were significant to health and were attributed to operational issues. In 2006 the NWHU published analyses of several water samples with regard to microbiological parameters, which revealed high counts of E. coli and total coliforms. NWHU (2006) indicated that such poor water quality was likely the result of using unclean pails when retrieving water, as analysis at the treatment plant indicated that water was treated sufficiently. At the time of Mayberry's report, Pikangikum had been subjected to a 3,285 day DWA.

Comparison of community drinking water quality. There is a minor difference between Red Lake and Pikangikum in terms of drinking water quality monitoring, such that former is in compliance with all required monitoring practices while the latter has one lapse. This difference is significant, as the monitoring practice is for microbiological parameters, which represents public drinking water sources' greatest risk to health (Hrudey et al., 2002). Pikangikum's non-compliance may be a direct consequence of the governance actors' change theories such that the AFN and CoO's change theory appeared to be the only one to not include monitoring. Further, $\mathrm{HC}$ employed EHOs have not 
travelled to the First Nation to help with monitoring practices. This is significant, as HC's change theory stresses the importance of monitoring throughout the drinking water system. Without substantiating such claims, $\mathrm{HC}$ is implicitly articulating to CBWMs and WTOs that monitoring in Pikangikum is not imperative. Therefore, the First Nation may not see monitoring as a priority of the change theory.

There are significant differences between Red Lake's and Pikangikum's drinking water quality, such that the latter's is not fit for human consumption despite treatment. This disparity is the result of the culmination of the differences between Red Lake's and Pikangikum's drinking water system design, infrastructure, and O\&M practices. Pikangikum is severely disadvantaged in each of these elements of the drinking water system such that it is unable to: distribute water to its residents; provide a sufficient quantity of quality drinking water to its residents; or secure funding for effectively treating drinking water and maintaining the water system. Indeed, these differences reflect gaps between the governance actors' change theories, primarily with respect to funding issues and were discussed previously ${ }^{35}$.

\section{Improvement Recommendations}

Red Lake. System improvements above and beyond standard O\&M for Red Lake are divisible into two categories, those that have been completed and those which are still recommended. A breakdown of incurred expenses is provided in Annex 19. Overall, the Red Lake drinking water system has incurred 12 major improvements beyond standard operation and maintenance practices totalling $\$ 371,300$. Six $(50.0 \%)$ of the expenditures pertained specifically to infrastructure investment and accounted for $\$ 273,891(73.8 \%)$ of

\footnotetext{
${ }^{35}$ While an argument could be made that current issues pertaining to microbiological contamination from using dirty pails is a result of a lack of public awareness, this would not be of importance if the drinking water system's larger issues were addressed.
} 
total costs. The remaining six (50\%) expenses incurred were with regard to O\&M, totalling $\$ 97,409(26.3 \%)$ of costs. Only one improvement is needed to bring the system into compliance with the Safe Drinking Water Act, 2002, such that the water tower must inspected and cleaned regularly (MOE, 2012c). It is also notable that the municipality has had issues with lead contamination due to the age of the distribution system, which may illustrate a future need for replacement (Northern Waterworks, 2011).

Pikangikum First Nation. Recommended improvements for Pikangikum's drinking water system can be divided into immediate improvements to ensure compliance with the Protocol and future improvements to ensure serviceability (Mayberry, 2010). An overview of immediate recommendations is provided in Annex 20. Mayberry makes 20 recommendations to fulfill the Protocol's requirements, totalling $\$ 3,292,500$. One $(5.0 \%)$ recommendation pertains to source water protection, accounting for $\$ 40,000(1.2 \%)$ of total costs. Ten $(50.0 \%)$ suggestions relate to infrastructure investments and totalling $\$ 3,132,000(95.1 \%)$ of costs. Two (10.0\%) recommendations related to training account for $\$ 15,000(0.4 \%)$ of the total. Five $(25.0 \%)$ suggestions pertain to O\&M, accounting for $\$ 85,000(2.6 \%)$ of costs. One $(5.0 \%)$ recommendation pertained to monitoring practices and $\$ 10,000(0.3 \%)$ of costs. One $(5.0 \%)$ recommendation regarded emergency contingency measures accounting for $\$ 10,000(0.3 \%)$ of the expenditures.

Mayberry (2010) also provides several improvement recommendations for future serviceability, which are with regard to establishing drinking water services on par with other Canadians living in northern Ontario. Mayberry provides five suggestions pertaining to developing a new water treatment plant and a piped distribution system, totalling $\$ 32,759,000$. Four ( $80 \%$ ) of these improvements are with regard to 
infrastructure investments and account for the majority of the costs, $\$ 32,318,000$

$(98.7 \%)$. The remaining expenditures regard annual O\&M costs that would be required with a new drinking water system and total $\$ 441,000(1.3 \%)^{36}$.

Comparison of community improvement recommendations. It is evident that there are significant differences pertaining to the improvements needed for Red Lake's and Pikangikum's drinking water systems for complying with the presiding regulations/guidelines. First, there are 19 more recommendations for Pikangikum to gain compliance with the Protocol than there are for Red Lake to comply with provincial regulations. Indeed, even when accounting for improvements that have already taken place, Pikangikum must still fulfill seven more recommendations than Red Lake has undertaken since 2008. Recommendations to service Pikangikum at a level similar to Red Lake total more than $\$ 30$ million. These differences between the communities are the result of funding arrangements and ongoing O\&M practices.

First, the funding arrangements for Red Lake are unambiguous relative to Pikangikum in that the former primarily relies on user-fees and the latter is reliant on AANDC for the majority of funding contributions. On the one hand, Red Lake has been able to obtain the appropriate funds to operate and maintain the system and has access to additional municipal funds should the need arise. Furthermore, Red Lake has applied for and received more than $\$ 700,000$ for infrastructure renewal from the MOI. On the other hand, Pikangikum does not currently have the infrastructure needed to provide a sufficient amount of quality drinking water nor does it have the infrastructure necessary

\footnotetext{
${ }^{36}$ Mayberry provides an option for expanding the number of watering points within the community, which requires much less investment $(\$ 3,552,000)$ than the recommended option; however, he dismisses this option, stating it will not allow Pikangikum to receive drinking water services analogous to other northern Ontarians.
} 
to distribute treated water to its residents. While the department committed to providing $\$ 9.7$ million for such projects in 2007 , this amount significantly less than the estimated $\$ 33$ million needed, according to Mayberry (2010). Even apart from the newly needed infrastructure, the First Nation would require more than $\$ 3$ million to bring its water system into compliance with the Protocol. Due to the fact that $95.1 \%$ (\$3.1 million) of recommended funds pertain to infrastructure needs, AANDC is responsible to provide the majority of this funding. This underfunding exemplifies a major gap in the governance actors' theories of change, such that First Nations believe that their drinking water systems are underfunded by AANDC, whereas the federal department suggests that First Nations do not efficiently use the funds that have been provided. Regardless of AADNC's concerns over First Nations' inefficiencies, the department is underfunding this community's drinking water quality. A small portion (1.8\%) of recommended funding totalling $\$ 60,500$ to meet the Protocol is also unallocated in the current governance actor role definitions. Without assigning funding responsibility to a governance actor, it is likely that the activity will go unfunded and not take place.

Second, because of Northern Waterworks' adherence to standard O\&M practices and Red Lake's sufficient funding of these activities, the drinking water system is likely to suffer few breakdowns and require minimal levels of new infrastructure. By comparison, Pikangikum's subpar efforts and lack of O\&M funding has likely contributed to the deterioration of the drinking water system, resulting in significant repairs and/or investments in new infrastructure. Thus, this explanation for the disparity in system improvements for Pikangikum and Red Lake again reflects differences in 
governance actors' funding philosophies, as demonstrated in AANDC's and the AFN and CoO's change theories.

The immediate suggested improvements for Pikangikum span across many of the activities listed in the governance actor's change theories including: source water protection; infrastructure investments; training; O\&M; monitoring practices; and water contamination crisis mitigation planning. This is in contrast to Red Lake, which incurred or was recommended improvements only with regard to new infrastructure and O\&M practices. This difference lends to the idea that Pikangikum's drinking water quality issues result from difficulty in the action theories of several specific interventions. This difference between Red Lake and Pikangikum illustrates that the First Nation requires a broad focus for remedying drinking water quality issues.

\section{Conclusion}

Comparisons of the various aspects of Red Lake's and Pikangikum's drinking water quality oversight illustrate a myriad of significant differences which suggest that Pikangikum is disadvantaged relative to Red Lake, including: financial capacity; the level of governance interest; and O\&M efforts. First, there is divergence between the level of funding available to Pikangikum and Red Lake. For instance, Red Lake's amalgamation into the Municipality of Red Lake has enabled the community access to a larger pool of financial resources that it otherwise would, which provides for greater flexibility and potential for economies of scale. Red Lake also primarily relies on user-fees to fund drinking water system on an as-use basis. By contrast, Pikangikum is reliant primarily on AANDC transfers, which have been deemed inadequate to sufficiently fund its drinking water system. Indeed, Pikangikum's infrastructure needs exceed \$3 million, as the 
community requires a distribution and a larger treatment facility. As noted throughout the chapter, difficulties with funding can likely be attributed to AANDC, which has been reluctant to supply adequate funds. Indeed, this is a reflection of the gap in the First Nation groups' and AANDC's change theories related to sufficient funding and efficiency.

Second, there is a difference in governance actor interest in Red Lake's and Pikangikum's drinking water systems. Red Lake is responsible for funding the operation of its drinking water system (through Northern Waterworks), the MOI provides additional funds, and the MOE regulates the system. There does not appear to be any gaps significant in these relationships. By comparison, AANDC has not provided adequate funding and has seemingly overlooked missing infrastructure and $\mathrm{HC}$ has been allegedly absent from overseeing monitoring practices. This lack of interest likely contributes to Pikangikum's continued drinking water quality issues, as the activities for which these governance actors are responsible go unfulfilled. Indeed, this may also articulates that the aspects for which they are responsible are unimportant for Pikangikum's drinking water system.

Third, Red Lake and Pikangikum also demonstrate a difference with regard to effort in O\&M practices. Specifically, Red Lake goes above and beyond legislation, whereas Pikangikum's efforts have been labelled subpar. Such differences in effort are likely the result of the aforementioned disinterest of governance actors. Indeed, regulations are actively enforced at Red Lake, while broad governance roles and responsibilities are unfulfilled in Pikangikum. 
A regulations gap has been apparent between Red Lake and Pikangikum, such that the latter is not subject to drinking water legislation. This difference is notable due to the primary premise of such regulations. Specifically, the First Nations drinking water governance actor most advocating for regulation is AANDC, which emphasizes that the need is for First Nations governments to be held accountable for spending funds. The irony here is that if regulations were in place and enforced by a regulatory body external to the governance actors, AANDC would likely be subject to the most significant recourse, indirectly through an increase in required funding. This idea lends to the EC interview respondent's suggestion that regulations could in fact help First Nations obtain needed funds.

Overall, the drinking water quality is significantly poorer at Pikangikum than Red Lake. There are a number of underlying issues that must addressed, beginning with governance actor interest and adherence to their respective roles. While the First Nation may be difficult to access, this is not a sufficient reason for the community to be overlooked by those actors responsible for ensuring quality drinking water that is safe to consume. 


\section{Chapter 7: Concluding Observations Regarding Drinking Water Quality}

\section{Differences between Analogous First Nations and Non-First Nations Communities}

This final chapter begins by responding to the three sub-questions, which guided the thesis.

(1) How do four federal and Aboriginal governance actors involved in managing drinking water on First Nations reserves define the problem regarding First Nations' drinking water quality?,

(2) How do these actors' solutions for remedying the problem differ; and, in what specific ways do they diverge? and,

(3) Are the differences in solutions for remedying drinking water quality issues on First Nations reserves contributing to the drinking water quality issues?

The first and second sub-questions are discussed briefly, as the bulk of analysis was overviewed in Chapter Three. The discussion of the third sub-question provides a summary of the major drinking water quality management differences identified in the three case studies. These differences are presented as major themes that are related back to the gaps between First Nations drinking water quality governance actors' theories of change (sub-questions one and two). The information presented within these themes is also augmented with Ontario First Nations data and data from the Ontario Ministry of the Environment (MOE). The rationale for the third section is to provide an overview of common differences, supplementing the data as appropriate, to enable generalizability. Although the provincial data are not matched on population size, water source, or location, the comparisons provide a high-level account of the divergence between the majority of First Nations and non-First Nations water systems in Ontario. Following 
these thematic summaries, a brief analysis of why these issues persist is presented. From this analysis, recommendations to remedy the differences between drinking water quality between First Nations and non-First Nations communities are presented. The chapter closes with a discussion regarding the limitations and implications of the research.

\section{Sub-Question1: Defining the Problem}

"How do the four federal and aboriginal governance actors involved in managing drinking water on First Nations reserves define the problem regarding First Nations' drinking water quality?"

It was explained in chapter two that three of the four governance actors (AANDC, $\mathrm{HC}$, and $\mathrm{AFN}$ and $\mathrm{CoO}$ ) identified the primary problem as an issue for which other governance actors were responsible. Although the fourth governance actor (EC) indicted that its own responsibility was a primary challenge, the department qualified this observations by suggesting that its role in First Nations drinking water quality governance is exaggerated by the other actors. That is, EC regards its responsibilities as a support role, rather than the focus of water management. Therefore, there is evidence to suggest that conflicting mandates and differing rationales for understanding and addressing water quality issues contributes to disparity between the governance actors' change theories. Specifically, each actor's primary solutions for addressing water quality are mutually dependent on other actors' contributions to drinking water quality governance. For example, instead of focusing on funding issues, AANDC's change theory takes aim at operating efficiency and operator training, which are the responsibilities of the First Nations communities? By contrast, the AFN's and CoO's theory of change focuses on resolving funding deficiencies rather than operating 
efficiencies. Therefore, no universal and mutually accepted change theory has been developed among the actors which allows for a comprehensive and coordinated solution.

\section{Sub-Question 2: Solutions for Remedying the Problem}

"How do these actors' solutions for remedying the problem differ; and, in what specific ways do they diverge?"

The governance actors' solutions for remedying the problem had similar elements but differed in implementation. The specific change models for each governance actor illustrated differences at almost every common element of their respective change theories. As anticipated, the differences could be attributed to the manner in which problems were defined according to each governance actor. Implementation was dependent on the results of the interpretation of specific governance mandates and responsibilities. For example, whereas the AFN and $\mathrm{CoO}$ suggested that AANDC should provide more funding for water quality activities, AANDC argues that water quality management must be made more efficient on reserves. Therefore, each of the actors is speaking at cross-purposes from each other resulting in zero-sum responses to the problems as they are identified and defined by each actor.

\section{Sub-Question 3: Divergent Solutions Contributing to the Problem}

"Are the differences in solutions for remedying drinking water quality issues on First Nations reserves contributing to the drinking water quality issues?"

The three matched-pair case studies demonstrate six major elements of divergence between governance actors' change theories and how they have contributed to prolonged water quality problems for First Nations relative to analogous non-First Nation communities. These include: 
- Financial capacity;

- Human resource capacity;

- Governance role clarity;

- Regulation and enforcement;

- Evaluation technique;

- Improvement recommendations; and

- Water source disparity.

Differences in financial capacity. Each of the three matched case-pairs illustrated that the First Nations communities have insufficient financial resources relative to non-First Nation communities. The First Nations communities required financial resources to fund several common areas of local drinking water management including: new infrastructure; operations and maintenance (O\&M) activities; water treatment operator (WTO) training; and drinking water quality monitoring practices.

First, there are inherent differences in the way the First Nations and non-First Nation communities obtained funding. Non-First Nations communities are funded mainly through user-fee systems. These fees are not uniform across the three non-First Nation communities, as each used a different combination of fixed and variable rates. They also vary in terms of amount, whereby one community utilizes a fully user-funded system whereas the others rely on a combination of municipal taxes, provincial government contributions, and user fees. By comparison, each of the First Nations communities relies on Aboriginal Affairs and Northern Development Canada (AANDC) for funding their drinking water systems. Funds are provided to First Nations in lump sum transfer payments annually. First Nations are also responsible for funding $20 \%$ of O\&M expenses from their own-source revenue. This is a common difference throughout Canada (Smith et al., 2006). 
This difference in funding models presents a myriad of issues for First Nations as AANDC's lump sum payments are insufficient for normal operation of drinking water systems (Mayberry, 2010; Paznar, 2011; Salma, 2010). This is concerning because AANDC's perspective is that funds are sufficient. Contrastingly, the Assembly of First Nations (AFN) and Chiefs of Ontario ( $\mathrm{CoO}$ ) maintain that funds must be increased to allow for normal operation of drinking water systems. According to the Neegan Burnside report (2011c), First Nations communities in Ontario require at least $\$ 242$ million in funding to meet the provisions within the Protocol for Centralised Drinking Water Systems in First Nations Communities (the Protocol) ${ }^{37}$. Of these recommended funds, $\$ 228$ million (94.3\%) must be allocated for infrastructure, and $\$ 4$ million (1.7\%) toward O\&M activities. Because First Nations communities have direct control over allocating O\&M spending but not infrastructure spending, there is some evidence to suggest there are few efficiencies First Nations can institute that can contribute to filling the gap between First Nations and non-First Nation communities. Specifically, the Ontario Chief Drinking Water Inspector (MOE, 201 la) suggests that improvements needed for Ontario communities to meet the Safe Drinking Water Act, 2002 relate to operations and maintenance activities. No indications of required funding are given. However, in the event non-First Nation communities have insufficient funds for operating their system, the community would able to raise user fees to obtain the funds needed. Based on the results of the matched-pair case studies, it is reasonable to assume that additional funding is significantly greater for First Nations than non-First Nations Ontario communities, as infrastructure costs are significantly greater than O\&M costs.

\footnotetext{
${ }^{37}$ On a national basis, immediate recommendations to meet the protocol total $\$ 861$ million (Neegan Burnside, $2011 \mathrm{~b}$ ). Of these costs, $\$ 783$ million (90.9\%) is with regard to infrastructure and $\$ 15$ million $(1.7 \%)$ is with regard to operations and maintenance.
} 
Second, the non-First Nation communities have been amalgamated into larger municipalities in response to provincial recommendations. Since 1996, the number of municipalities has decreased from 815 to 444 in Ontario [Association of Municipalities (AMO), 2012], meaning that they now have access to greater pooled human and financial resources.

Differences in human resource capacity. First Nations and non-First Nations communities' human resource capacity differences for effectively managing their drinking water systems is pronounced. First, due to non-First Nations community amalgamations, drinking water systems are managed by sizable staffs. For example, the Municipality of Greater Sudbury manages a staff of almost 100 people, including 13 WTOs, who contribute to the management of the Dowling drinking water system. By comparison, Sagamok manages a staff of eight individuals (including three WTOs). It is clear that First Nations appear more likely to suffer challenges in management especially with respect to regulatory compliance.

A second difference related to human resource capacity is WTO training. Only one WTO in the First Nations communities studied was sufficiently certified to work on his or her community's drinking water systems. However, this training was only to the first certification level, which was considered sufficient for Sagamok's groundwater system. No First Nations secondary WTOs had any certification training. Only $45 \%$ of primary WTOs and 13\% of WTOs have obtained adequate certification training in Ontario (Neegan Burnside, $2011 \mathrm{c})^{38}$. By contrast, Ontario municipal systems are required to have trained primary WTOs and secondary WTOs as set out by the Safe Drinking

\footnotetext{
${ }^{38}$ Nationally, only $54 \%$ of First Nations treatment plants employ sufficiently certified primary WTOs (Neegan Burnside, 2011b). Three percent of First Nations treatment plants have no WTO and 19\% have no secondary operators.
} 
Water Act, 2002. According to the Chief Drinking Water Inspector, there are approximately " 6,213 certified drinking water operators in Ontario holding 8,502 certificates" (MOE, 201 la), meaning that there is an adequate number of WTOs for the 693 drinking water systems in Ontario. This difference between First Nations and nonFirst Nations communities is of significant concern, as "certified operators are more likely to operate facilities in compliance with applicable guidelines and legislation" (Neegan Burnside, $2011 \mathrm{~b}$ ).

In each of the three matched-pair case studies, the differences in WTO training are attributed, at least in part, to funding issues. The funds required for sufficient WTO training in Ontario is approximately $\$ 1.7$ million (Neegan Burnside, $2011 \mathrm{c}$ ). On a national level the amount of funding required is approximately $\$ 9$ million (Neegan Burnside, $2011 \mathrm{a} ; 2011 \mathrm{c} ; 2011 \mathrm{~d} ; 201$ le; 201 le; $2011 \mathrm{f} ; 2011 \mathrm{~g} ; 201$ lh). This requirement is intriguing, particularly when considering that AANDC has committed to providing $\$ 10$ million per year to the Circuit Rider Training Programs (AADNC, 201 le). As noted, these programs are administered by provinces and non-government organizations to provide certification training to WTOs. It is also pertinent to note that the only observable compliance mechanism for AANDC to ensure that First Nations adhere to provisions within the Protocol is to withhold funds from First Nations without adequately trained WTOs. There is evidence to suggest that this option is rarely utilized. Further, there is a disincentive for First Nations to ensure that their WTOs are trained, because any increase in credentials would require increased salaries and benefits and further tax their AANDC funding base. In addition, the AFN and CoO, Environment Canada (EC), and Health Canada (HC) articulated that non-First Nations communities poach WTOs from First 
Nations once they are trained as such training is considered a subsidy for municipalities. Therefore, it is apparent that without additional funding for O\&M activities (WTO wages) and a practical compliance mechanism, this issue of untrained WTOs will persist. Differences in governance role clarity. The three case studies demonstrate that there are significant differences in the clarity of governance roles in overseeing drinking water quality for First Nations and non-First Nations communities. Specifically, non-First Nation communities have a comprehensive understanding of the roles and responsibilities of MOE and the municipality. The province regulates the activities of communities through clear standards under the Safe Drinking Water Act, 2002. By comparison, there is significant role ambiguity for the First Nations governance actors. Although water management activities are well known ${ }^{39}$, there is ambiguity surrounding responsibilities for activities that fall outside of the basic elements of a drinking water system.

The cases illustrate that there may be confusion with regard to specific funding responsibilities that fall outside of standard O\&M practices and infrastructure investments. As noted, these costs account for $95.9 \%$ ( $\$ 232$ million) of the total $\$ 242$ million required for Ontario First Nations to immediately meet the requirements of the Protocol. Therefore, $\$ 10$ million (4.1\%) of such costs are not specifically assigned to any one governance actor. Due to lack of clarity around roles and responsibilities between AANDC, HC, EC, and First Nations, it is unlikely that any governance actor will voluntarily assume responsibility for those activities not readily spelled out.

\footnotetext{
${ }^{39}$ AANDC is responsible for funding infrastructure, training, and $80 \%$ of O\&M activities; First Nations directly manage and monitor the system as well as fund $20 \%$ of O\&M activities; HC oversees water quality monitoring programs and is responsible for training WTOs; and EC provides advice to First Nations on source water protection
} 
An example of governance role ambiguity between First Nations and non-First Nations communities relates to source water protection and hydrogeological studies. Both Dowling and Sagamok were recommended to conduct a hydrogeological study to determine whether their source water required protection for the direct influence of surface water. Dowling, like all Ontario communities relying on groundwater, was required to fund the study and provide the results to the MOE. By contrast, Sagamok has yet to complete the study, despite the responsibility set out within the Protocol and Paznar's report to Neegan Burnside. Indeed, this is a common issue amongst First Nations communities in Ontario, as $75.2 \%$ (29) of communities relying on groundwater still require a hydrogeological study to be conducted ${ }^{40}$ (Neegan Burnside, 2011c). The role clarity for First Nations has become an issue in this regard because AANDC does not provide funding for hydrogeological studies, which would leave one to assume that such funding is the First Nations responsibility. Given that funds are already limited for infrastructure, human resources, and O\&M, it is unlikely that the First Nations will have sufficient funds to address source water protection.

The example demonstrates how a lack of role definition may result in responsibilities being passed on to First Nations, especially those that fall between the cracks of those responsibilities. This scenario was an articulated concern for the AFN and $\mathrm{CoO}$, as the interview respondent stated that role ambiguity would result in responsibilities being offloaded to First Nations (personal communication, April 4, 2012). EC suggested that its responsibilities with regard to source water protection have often been exaggerated by the other governance actors, such that it only participates in

\footnotetext{
${ }^{40}$ Nationally, 55.6\% (209) of First Nations' drinking water systems reliant on groundwater sources require a hydrogeological study to determine if they are under the influence of surface water (Neegan Burnside, 2011b)
} 
providing advice to First Nations with regard to this activity whereas other actors assume EC engages in active participation. This misunderstanding will likely leave such activities underfunded and unfulfilled. A similar example of role ambiguity leading to additional First Nations responsibilities may be demonstrated through HC's lack of active participation in the monitoring practices at Pikangikum. That is, the responsibility of monitoring may be overlooked due to HC's absence, particularly considering that the department is responsible for tracking compliance with monitoring practices prescribed by the Procedure Manual for Safe Drinking Water in First Nations Communities South of $60^{\circ}$. Together these examples illustrate that role definition is a concern for the management of effective First Nations drinking water systems.

Differences in evaluation. There were discrepancies between the drinking water system evaluation methods for the First Nations and the non-First Nation communities studied. Such discrepancies could lead to false conclusions about differences in drinking water quality and contamination risk. For instance, evaluation discrepancy occurs when comparing drinking water quality measures, as the First Nations were required to monitor more drinking water parameters than the non-First Nations, which means that the probability of a quality breach is higher for First Nations. Therefore, First Nations communities are more likely to be evaluated as having poorer drinking water quality than non-First Nations.

The difference in parameters monitored also becomes problematic when considering First Nations that rely on a municipal transfer agreement (MTA). For instance, Ginoogaming was assessed as having a low chlorine residual for ensuring effective treatment; however, it was the same standard to which Longlac was required to 
comply. Therefore, because the GCDWQ is more stringent than the Safe Drinking Water Act, 2002, exceedences (and treatment residual issues) of First Nations may be overstated relative to non-First Nations communities.

There are also discrepancies in system design evaluation. Under the Safe Drinking Water Act, 2002 regulations regarding appropriate infrastructure are in place to determine ongoing compliance, whereas AANDC only requires that the infrastructure currently in place complies with the Protocol. The issue with AANDC's evaluation method is that it does not account for communities without drinking water systems, which is precisely what occurred with Pikangikum's distribution system. That is, the Pikangikum system was assessed as not having system design issues because there was no infrastructure. In its national overview report, Neegan Burnside (2011b) observed that positive system design evaluations frequently showed false positives for a number of First Nations communities and recommended remedying this issue by amending the evaluation method to account of the lack of infrastructure within the evaluation ${ }^{41}$. An additional issue that is directly linked with evaluating distribution systems falsely is that daily drinking water demands are under-estimated. Again, this occurred at Pikangikum, as residents demanded less water than they otherwise would have with a fully implemented distribution system. Indeed, if a sufficient distribution system was in place, the capacity of the treatment plant would not have been assessed as meeting design requirements of the Protocol.

Overall, discrepancies in the evaluation methodologies may lead to significant differences in the assessment of drinking water quality issues for First Nations relative to non-First Nations communities. As a result, comparing these evaluations may overstate

\footnotetext{
${ }^{41}$ Nationally, $19.6 \%$ (158) of all First Nations water systems still make direct use of raw water as their drinking water source due to a lack of infrastructure (Neegan Burnside, $201 \mathrm{lb}$ ).
} 
disparity between the specific measures used for First Nations drinking water quality relative to those of non-First Nations. However, evaluation differences that understate the disparity in terms of infrastructure could have drastic long-term effects on drinking water quality and risk of contamination if system design issues are not addressed adequately. The major issue that may need to be addressed is a potential conflict of interest whereby AANDC is responsible for developing the evaluation methodologies for First Nations and is also responsible for funding infrastructure. By comparison, the MOE establishes evaluation methodologies for drinking water in Ontario communities and the communities are responsible for funding infrastructure issues. This difference between First Nations and non-First Nations communities is increasingly of concern when considering the responsibilities between AANDC and First Nations. That is, the AFN and $\mathrm{CoO}$ recommend additional funding to ensure that ongoing capacity is maintained. By contrast, AANDC suggests that current funding is sufficient for First Nations to operate drinking water systems, and that if First Nations are more efficient, then water quality can be achieved on par with non-First Nations. First Nations are reliant on AANDC transfer payments and until either of these attitudes is affected First Nations will continue to receive similar funding, which does not accurately reflect infrastructure needs.

Differences in regulation. There is a disparity of drinking water quality regulation between the First Nations and the non-First Nations communities studied, as the former have no regulatory regime in place and the latter are subject to rigorous standards. The only real regulatory mechanism available for the governance actors of the First Nations is withholding transfer payments for non-compliance with the Protocol, but such action is rarely taken because to do so would further diminish First Nations' abilities 
to provide quality drinking water to their residents. No regulatory mechanisms exist to ensure the other three governance actors (i.e., AANDC, EC, and HC) are adequately fulfilling their roles and responsibilities. Indeed, the only form of enforcement power is held by AANDC. By comparison to the First Nations, the non-First Nations studied were all subject to regulation under Ontario's Safe Drinking Water Act, 2002. Such regulations are enforced by the MOE, a provincial ministry external to the management and funding of drinking water systems.

The regulatory gap is significant as it is an important element of a comprehensive change theory. However, there is disagreement as to when and how regulations should be implemented and who should enforce them. AANDC suggests that regulations are implemented as soon as possible, whereas the remaining three governance actors recommend that they occur once First Nations have the human and financial resources capacity to effectively comply with them. The AFN and CoO suggest that the establishment of a First Nations water commission that would regulate the activities related to drinking water quality. Alternatively, AANDC and $\mathrm{HC}$ recommend that $\mathrm{EC}$ be charged with regulation, as it already regulates wastewater in many jurisdictions. In order to have comparable standards, regulations would have to be implemented immediately, enforced by an external party, and overseen by all four First Nations drinking water quality governance actors.

Differences in improvement recommendations. The case studies illustrated that First Nations communities were subject to more drinking water system improvement recommendations relative to the non-First Nations communities. Five activities discussed in chapter two were prevalent in the immediate improvement recommendations of all 
three First Nations communities studied including: source water protection; infrastructure investment; operator training; operations and maintenance; and mitigating against contamination emergencies. Two other activities, research and water quality monitoring, were included in two of the three First Nations cases studied. Non-First Nation improvements were mainly related to infrastructure and O\&M. Estimated expenses were much higher for First Nations' water systems than non-First Nations' systems. It was observed, therefore, that First Nations were subject to a wider array of system improvements than their non-First Nation counterparts.

On a national level, recommendations for improving First Nations drinking water systems have been similarly broad in scope as the case studies analyzed. Neegan Burnside (2011b) summarizes the most common system improvements needed to meet the Protocol through 12 overarching recommendations, including the following (verbatim):

- Provide minimum required level of treatment including disinfection and adequate contact time as required;

- Provide basic service level for existing non serviced homes;

- Conduct GUDI investigations where necessary;

- Provide required monitoring equipment and alarm systems;

- Provide standby power;

- Provide identified backup equipment to improve system reliability;

- All other identified [infrastructure] works and measures;

- Increase support of Circuit Rider Training Program;

- Ensure systems have a certified primary and backup operator;

- Enhance awareness and follow-up to encourage adequate monitoring and record keeping

- Develop and promote templates for source water protection plans, emergency response plans and maintenance management plans; and

- It is recommended that [AANDC] review and clarify some of the tools used in assessment of the water and wastewater systems, including the protocols and design guidelines, and the risk analysis system 
These recommendations relate to eight activities provided by the governance actors including:

- Operations and maintenance;

- Infrastructure;

- Research;

- Source water protection;

- Training;

- Monitoring; and

- Emergency mitigation.

By contrast, the Chief Drinking Water Inspector (MOE, 201 la) identifies only four common major system improvements for Ontario municipal drinking water systems to undertake, including (verbatim):

- Operating treatment equipment;

- Having up-to-date operation and maintenance manuals;

- Operating chlorination equipment; and

- Following proper procedures with regards to verbal notifications of adverse water quality incidents.

Three of these system improvements pertain to operations and maintenance activities.

The fourth regards mitigating against water contamination events. Generally, First Nations communities have more obligations to meet the provisions of the Protocol relative to Ontario municipal drinking water systems attainment of the standards within the Safe Drinking Water Act, 2002.

This difference in improvement recommendations reflects multiple gaps between the First Nations drinking water quality governance actors' responsibilities. There are gaps at nearly every element of even the common activities. These gaps are likely leading to the dispersed focus, thereby prolonging the corresponding issues of drinking water quality of First Nations. For example, funding for a variety of activities identified by AANDC and the AFN and $\mathrm{CoO}$ (e.g., infrastructure, O\&M, training, source water 
protection) are contested such that AANDC believes that federal funding is sufficient and the $\mathrm{AFN}$ and $\mathrm{CoO}$ maintain that funding must increase. The issues pertaining to drinking water quality and potential for contamination are prolonged, as long as these gaps between the governance actors' change theories persist.

Water source disparity. As anticipated, the case chapters provided evidence to suggest that the difference between First Nations and non-First Nations drinking water management is exacerbated by the complexity of the water system. Specifically, the differences within case studies were most pronounced for the communities relying on surface water as their primary drinking water source. As noted in chapters one and two, surface water is the most complex type of water source to effectively manage and treat. The disparity between Pikangikum and Red Lake relative to the other cases provides evidence that First Nations are less equipped to provide quality drinking water than nonFirst Nations as systems become more complex. Indeed, the difference in recommendations between the groundwater cases relative to the MTA cases provide further evidence to suggest that the complexity of the system increases the disparity of First Nations and non-First Nations management of drinking water quality.

\section{Recommendations}

The evidence provided in the thesis answers the main research question: "Does the complex management framework of drinking water systems on First Nations reserves contribute to the issues of drinking water quality in these communities?"

The contributions (or lack thereof) from the four independent actors create a First Nations drinking water governance structure that is divergent from non-First Nation communities. There is a problem of non-collaboration between the actors whereby, 
instead of engagement and the development of a coordinated and comprehensive change theory, four independent mandates with independent approaches to solutions have emerged which place the onus of water quality responsibility on other actors. In order to come to a solution for remedying the problem of First Nations drinking water quality, these actors must develop a common problem definition and together build a coordinated approach. The following recommendations are devised to foster such a process.

Recommendation 1: Improving trust. It seems that the most important issue to resolve is an underlying issue of confidence between the governance actors. Each of the actors has indicated that other actors are at fault for the challenges being faced by First Nations in the area of water quality. EC, for example, explains that the other governance actors do not understand the limitations of their role. The lack of confidence becomes apparent when reviewing discussions respecting treaty rights.

First Nations groups (i.e., the AFN and $\mathrm{CoO}$ ) are adamant that the federal government has a fiduciary responsibility to consult with First Nations on all decisions pertaining to First Nations communities and particularly water resources. According to the Delgamuukw v. British Columbia, 1997 ruling, the Crown is obligated to consult with affected First Nations when the federal government makes decisions that will impact these First Nations' rights (Hurley, 2000). In the spirit of these Supreme Court rulings, an argument can be made for a more comprehensive definition of consultation and engagement that can be agreed by all governance actors. This is a matter that transcends water management, but it is notable that First Nations and the governance actors raise it in this context. Such a step will provide a stronger foundation to build relationships and 
subsequent problem definitions and change theories, provided that these rights are considered continually.

Overall, building confidence is likely only through adequate communication. It must be determined precisely what level of engagement is needed at each step of a comprehensive and coordinated solution. It is unlikely that full engagement of all actors will occur for all activities, but consensus must be the focus of such engagement. Although AANDC has articulated that it has engaged First Nations in the past, particularly with regard to the development of legislation, First Nations groups have been dissatisfied with the scope of such engagement. The AFN and $\mathrm{CoO}$ articulated that such engagement often seem fruitless, as AANDC appears to make unilateral decisions regardless of the engagement offered. Instead, it is recommended that comprehensive planning for engagement take place to enable all voices to be heard and to contribute to a democratically developed problem definition and subsequent change theory. This recommendation is in line with engagement recommendations made by both the Standing Senate Committee on Aboriginal Peoples (St. Germain, 2007) and implied by the Expert Panel on Safe Drinking Water for First Nations (Swain, Louttit, \& Hrudey, 2006). However, this recommendation goes beyond broadly suggesting regulation to include a target: Developing a comprehensive and democratically coordinated problem definition and theory of change.

Recommendation 2: Improving role definition. A second recommendation is to develop substantial role definitions for each governance actor, and to ensure that activities are assigned appropriately. Role definitions and responsibility assignments 
should be agreed upon by all governance actors in order to build a comprehensive and coordinated theory of change.

First, it has been made clear that EC's role in source water protection is often exaggerated by the other governance actors. As a result, source water protection is often underfunded and unaddressed. Clear role definitions would ensure that the funding and activities related to source water protection would be assigned to one or more specific actors. Non-compliance would then be easier to evaluate and remedy.

Second, there is ambiguity in relation to governance actors' responsibilities for funding non-infrastructure and non-operations and maintenance costs. Infrastructure and O\&M costs are assigned specifically to AANDC and the First Nations. Additional costs apart from these categories, such as those relating to hydrogeological studies, research, emergency planning, and source water protection, are left without a specific actor providing funds for associated activities. This gap must be addressed, so as to improve water management evaluation, including better defining performance measures.

Overall, clearer roles and responsibilities have been a common recommendation of most expert reports including the Standing Senate Committee on Aboriginal Peoples (St. Germain, 2007), implied by the Expert Panel on Safe Drinking Water for First Nations (Swain, Louttit, \& Hrudey, 2006), and the National Assessment of First Nations Water and Wastewater Systems (Neegan Burnside, 2011b). The Report of the Commissioner of the Environment and Sustainable Development argues that role definition, clarifying responsibilities, and developing comprehensive guidelines, policies, and standards is a top priority that is going unaddressed (Office of the Auditor General, 2005). However, these reports do not specify how specific responsibilities should be 
determined and assigned. The method proposed here is democratic decision making, whereby confidence must first be built for roles to be sufficiently defined in such a way that the actors are willing to assume the associated responsibilities.

Recommendation 3: Improve coordination. A third recommendation is to improve coordination amongst the governance actors. For instance, coordinating funding activities is essential in order to better manage water issues on reserves. Another example occurred in two of the three case studies where HC's water quality data was not readily available for the AANDC financed National Assessment of First Nations Water and Wastewater Systems. This data was not made available in Pikangikum due to a $\mathrm{HC}$ oversight. Similarly, Sagamok did not approve the release of its system data. Therefore, coordination is even lacking within a significant, well-planned national assessment of First Nations. In order to improve drinking water quality of First Nations communities, better coordination between all responsible governance actors is required within each activity of a comprehensive change theory.

A component of coordination may include regulation, which has been a recommendation of several expert reports including the Standing Senate Committee on Aboriginal Peoples (St. Germain, 2007) and implied by the Expert Panel on Safe Drinking Water for First Nations (Swain, Louttit, \& Hrudey, 2006). As noted, Swain et al. have suggested that capacity must be established prior to the development of any regulation. In contrast, it is suggested here that regulations can be enforced to support First Nations capacity building. However, to be effective early, regulation must take place so as to include the responsibilities of all actors. The placement of these recommended regulations is consistent with those of AANDC. Although the scope of 
their regulations is far more broad than any of the other actors, the idea is that regulating all agreed upon roles and responsibilities will ensure compliance and better facilitate inter-actor coordination. As with all other recommendations thus far, these regulations should be set utilizing a democratic intervention method. Finally, it would be imperative for an external party to enforce the regulations, be it another federal department, provincial departments, or a First Nations water commission. This group must be selected through the same engagement process indicated and should be heavily involved in the development of those regulations.

\section{Considerations Moving Forward}

As noted above, the thesis recommendations were devised to clarify problem definition for the four actors, and contribute to the development of a comprehensive and coordinated solution for resolving drinking water quality issues in First Nations communities. However, these recommendations do not offer specific points of consideration for developing a problem definition and/or coordinated solution, but merely provide evidence to support specific activities that could be incorporated into an agreed upon problem definition and/or coordinated solution. Such evidence provides support for restructuring funding relationships, amalgamating drinking water services, and restructuring funder and compliance monitoring roles.

Consideration 1: Restructure funding relationships. It was evident throughout the case study chapters that First Nations did not have sufficient government funds available for operating and maintaining drinking water systems as analogous non-First Nations communities. The common theme presented was that there was a significant difference between AANDC and the First Nations' perceptions: AANDC believed funds 
were not being utilized efficiently; and, that First Nations suggest that funds are insufficient. The use of user-fees in non-First Nations communities supports selfsufficient drinking water systems (with the exception of Red Lake, which was supplemented). Such an idea may be considered by First Nations, but clearly allowances must be made for community capacity, political realities, and community willingness to contribute. These considerations are supported by Smith et al. (2006) who suggest that the "divorce between user-fees and responsibility of the system is an issue that requires serious attention". Indeed, it is likely that user-fees will provide more flexibility for First Nations to operate and maintain the drinking water system, as user-fees can be more quickly adjusted for addressing challenges as they arise than strict funding formula used by AANDC.

It would also be beneficial to review the financial capacity of each First Nation by incorporating own-source revenue. Specifically, it does not appear that a blanket policy requiring all First Nations to cover $20 \%$ of operations and maintenance funding could be effective or appropriate for all First Nations, when considering the diversity of ownsource revenue and socioeconomic conditions. Perhaps a new funding formula based on risk and capacity to pay could be considered by federal departments and communities. The model used by Red Lake (i.e., the municipality is responsible for funds, primarily through user-fees, and the provincial government provides funds to support infrastructure development), would better enable those first Nations with sufficient capacity to develop stronger drinking water quality.

\section{Consideration 2: Amalgamating drinking water services. Amalgamating}

drinking water services should be considered wherever possible, as significant 
differences were prevalent throughout the case studies. Each case study comparison illustrated that the non-First Nations communities were amalgamated into larger municipalities. These communities had the flexibility and economies of scale to pool funding and expertise. Consequently, each of these communities had sufficient funds for operating and maintaining their drinking water systems while being able to address issues as they arise. Similarly, they were able to maintain the required number of WTOs with sufficient training. The consideration here should not be to completely amalgamate First Nations communities with other First Nations and/or non-First Nations communities, but rather to amalgamate drinking water services similar to that of MTAs. Specifically, it would serve to benefit drinking water quality to, wherever possible, consolidate the drinking water systems of First Nations with other First Nations and non-First Nations communities in proximity. According to Graham (2003), approximately 10,000 households are required to sustain a strong drinking water system. As such, amalgamating drinking water systems would help to provide sufficient human and financial resources for operating and maintaining the systems. This consideration could also take into account ideas of incentives to promote these amalgamations. ${ }^{42}$

\section{Consideration 3: Restructuring funder and compliance monitoring roles.}

There is a clear conflict of interest for the compliance monitoring and funding roles within First Nations communities relative to non-First Nations communities. Specifically, there is a clear divide between funder and regulator roles in the non-First Nations communities studied, such that the MOE is the regulator and the municipality is the funder. When additional funds were provided to Red Lake these were provided by the

\footnotetext{
${ }^{42}$ This consideration should also ensure that First Nations communities entering into MTAs are charged user-fees comparable to those of the other participating communities.
} 
Ministry of Infrastructure, a provincial department separate from the MOE. By contrast, funds in First Nations communities are primarily provided by AANDC, which is also responsible for monitoring compliance with the Protocol and utilizing enforcement mechanisms when possible. It is clear that there may arise a conflict between these roles. For example, the department could exercise bias when interpreting First Nations noncompliance with the Protocol, and considering the efficiency with which First Nations use funding or the availability of funding in general. Therefore, it seems that it would best serve drinking water quality in First Nations if these roles were separated and/or an external body could review the information independently. The use of an alternate department or external body could also assist in the development of a problem definition and a subsequent coordinated solution to drinking water quality issues. This consideration was expressed by the Expert Panel on Safe Drinking Water for First Nations in relation to enforcing regulations. However, this consideration goes beyond regulations and accounts for current compliance monitoring practices without regulations in place.

\section{Limitations}

The thesis was exploratory in nature and investigated apparent theories of change for addressing First Nations drinking water quality by drawing conclusions from the written and verbal discourse of the responsible four governance actors. The explicit ordering and step-by-step approach of these change theories reflected the perception of an outside party (i.e., the author) and therefore, may not have fully depicted the implicit intervention strategies held as accurate by each actor. Therefore, the conclusions drawn within the discussions and comparisons of change theories were inherently limited. Further 
investigation is warranted to corroborate the perceptions of the author to ensure the testretest reliability of this research.

Budget statements and the details of specific water quality problem were not analyzed in detail as such an analysis was beyond the scope of the thesis project. The information analyzed was instead a high-level account of change theories and interventions, which has led to similarly broad conclusions and recommendations for improving First Nations drinking water quality. Attempting to obtain such information is also quite challenging, as each First Nations community enters its own funding agreement with AANDC. ${ }^{43}$

Only three matched-pair case studies, each from northern Ontario, were utilized for the purposes of this study. The number of analogous communities studied limits the generalizability of the results for several reasons. First, there are unique relationships between each First Nation community and the other governance actors including aspects of funding, communication, and deference. Therefore, the conclusions drawn from the three matched-pair case studies may not accurately reflect the range of relationships between the First Nations and the other actors, nor can they be adequately generalized.

Given that only Ontario communities were examined, the prescribed drinking water quality measures of other provinces were not applied. As noted, the Protocol stipulates that First Nations are subject to the stricter standard of the Protocol or the provincial drinking water quality measure. Due to the fact that Ontario has the most rigorous drinking water regulations in Canada, the First Nations studied are subject to some of the strictest guidelines available. Therefore, First Nations in other provinces

\footnotetext{
${ }^{43}$ Freedom of information requests for budget details with regard to AANDC contributions toward drinking water in the three First Nations communities analyzed were returned with a statement indicating that contributions are highly complex and difficult to summarize. Detailed contributions were thus not provided.
} 
follow less rigorous guidelines, which may result in these communities being closer to compliance with the more relaxed standard relative to non-First Nations communities. Conversely, this difference may mean that First Nations communities in other provinces are significantly less apt for managing drinking water quality than those in Ontario.

Finally, the matched-pair cases used may not accurately reflect the challenges of non-First Nations communities, as Ontario currently upholds the most stringent provincial drinking water standards. Therefore, non-First Nations communities outside of Ontario may demonstrate similar challenges to analogous First Nations communities in provinces where provincial standards are limited. Overall, further investigation of more First Nations and analogous non-First Nations communities is warranted to promote or challenge the generalizability of the thesis findings.

\section{Implications and Conclusions}

First, the four governance actors identified differ significantly from the outset of their approaches to First Nations drinking water quality management. This fundamental difference begins at problem definition, which leads to divergent (and in some regards, opposed) solutions for remedying challenges to quality drinking water. The governance actors' solutions offer no singular desired outcome that they can work together to achieve. As a result, specific activities are inconsistently articulated and applied by these governance actors, which has contributed to the persistence of the challenges they are meant to remedy. By determining the starting point of divergence between the governance actors, this thesis may be beneficial articulating the need to start reevaluating the current process and establish a new comprehensive problem definition and 
change theory using a collaborative approach. Until this collaboration is completed, it is likely that piecemeal efforts will continue to enable the problems identified to persist.

Second, stemming from the divergence of the four governance actors and inconsistent activities, drinking water quality of the First Nations communities studied is of poorer quality (or at greater risk to become poor quality) than analogous non-First Nations communities. There is evidence to suggest management framework of the First Nations drinking water quality has a detrimental impact on these communities' drinking water relative to non-First Nations of similar size, location, and water source, which corroborates the anecdotal evidence many experts (O'Connor, 2002b; SDWF, 2011; Swain, Louttit, \& Hrudey, 2006). This finding is significant, as no other study has directly compared First Nations drinking water quality with analogous non-First Nations. Therefore, the thesis provides evidence that a different approach to must be taken to resolve First Nations drinking water quality issues than analogous non-First Nations, as the problems differ in type and severity.

Third, although the National Assessment of First Nations Water and Wastewater Systems (Neegan Burnside) points toward increasing funding as the primary component for remedying the gap between First Nations and non-First Nations, the thesis illustrates that this is only a portion of the problem. Indeed, the thesis provides evidence to suggest that there are many underlying problems that must be addressed prior to specific activities, such as increasing funding. The primary problem must first be defined to determine the best solution. Increased coordination is essential not only to the overall problem definition and solution, but also to those specific activities once the time is appropriate. For example, funding will be more likely to be appropriately and sufficiently 
allocated if clear roles and responsibilities are established through a comprehensive and coordinated solution.

Finally, the conclusions and recommendations of the thesis are made explicitly with regard to First Nations drinking water quality; however, the ambiguity and disagreement among the governance actors are likely not isolated to drinking water quality issues. Indeed, the research question of this thesis could be applied to any number of social issues apparent in First Nations communities. It follows that similar divergence in roles and responsibilities between federal and Aboriginal governance actors likely leads to the persistence of many similar issues. Therefore, the approach of this thesis may provide a useful evaluative tool for studying other such issues. 


\section{References}

Aboriginal Affairs and Northern Development Canada (AANDC). (2010). Transfer Payments. Retrieved from http://www.aadnc-aandc.gc.ca/eng/1100100010035.

AANDC. (2011a). Fact Sheet - Infrastructure Investments and Investment Planning. Retrieved from http://www.aadnc-aandc.gc.ca/eng/1313688016644.

AANDC. (2011b). Fact Sheet - Water Quality. Retrieved from http://www.aadncaandc.gc.ca/eng/1100100035034.

AANDC. (2011c). Frequently Asked Questions - National Assessment of Water and Wastewater Systems in First Nation Communities: Release of Final Reports. Retrieved from http://www.aadnc-aandc.gc.ca/eng/1313691954022.

AANDC. (2011d). Fact Sheet - Risk Assessment of Water and Wastewater Systems in First Nations Communities. Retrieved from http://www.aadncaandc.gc.ca/eng/1313687144247

AANDC. (2011e). Fact Sheet - Capacity and Training and Circuit Rider Training Program. Retrieved from http://www.aadnc-aandc.gc.ca/eng/1313688917297.

AANDC. (2011f). Frequently Asked Questions: Water School Kit for First Nations Children. Retrieved from http://www.aadnc-aandc.gc.ca/aiarch/mr/nr/j-a2007/22854-faq-eng.asp.

AANDC. (2011g). Funding Agreement National Model for Use with Aboriginal Recipients: Version for First Nations and Tribal Councils for 2011-2012.

AANDC. (2012a). About AANDC. Retrieved from http://www.aadncaandc.gc.ca/eng/1100100010023/.

AANDC. (2012b). Frequently Asked Questions - Safe Drinking Water for First Nations Act. Retrieved from http://www.aadnc-aandc.gc.ca/eng/1330528850798

AANDC. (2012c). Governance. Retrieved from http://pse5-esd5.aincinac.gc.ca/fnp/Main/Search/FNGovernance.aspx?BAND_NUMBER=208\&lang= eng.

AFN. (2008b). Letter from National Chief to Minister Strahl Regarding the Continued Unsatisfactory State of Access to Safe Drinking Water Experienced by Many First Nations People. Retrieved from http://www.afn.ca/uploads/files/water/08-0322.pdf.

AFN. (2009). Aboriginal Water Rights Primer. Retrieved from http://www.afn.ca/uploads/files/water-primer.pdf 
AFN. (2010a). Letter from National Chief Atleo to the Standing Committee on Aboriginal Peoples Regarding An Act Respecting the Safety of Drinking Water on First Nation Lands (Bill S-11). Retrieved from http://www.afn.ca/uploads/files/water/10-09-30.pdf.

AFN. (2010b). AFN News Bulletin-AFN National Chief Calls for Real Action on Safe Drinking Water for First Nations: Need Action to Address the "Capacity Gap as well as the Regulatory Gap". Retrieved from http:/www.afn.ca/uploads/files/water/10-05-27.pdf.

AFN. (2010c). Briefing note: Bill S-11, Safe drinking water for First Nations Act. Retrieved from http://www.afn.ca/uploads/files/bill-s-11.pdf.

AFN. (2010d). Resolution: Bill S-11, Safe drinking water for First Nations Act. Retrieved from http://www.afn.ca/uploads/files/water/10-12-14.pdf.

AFN. (2010e). Canada-Wide Strategy for the Management of Municipal Wastewater Effluent and Environment Canada's Proposed Regulatory Framework for Wastewater: Impacts for First Nations Communities. Retrieved from http://www.afn.ca/uploads/files/regional-impact-analysis.pdf

AFN. (2011). National Chief March 8 letter to First Nations on Bill S-11. Retrieved from: http://www.afn.ca/index.php/en/news-media/current-issues/national-chiefmarch-8-letter-to-first-nations-on-bill-s-1 1-safe-drinking-w

AFN. (2012a). Description of the AFN. Retrieved from http://www.afn.ca/index.php/en/about-afn/description-of-the-afn.

AFN. (2012b). Water and Wastewater. Retrieved from http://www.afn.ca/index.php/en/policy-areas/water.

Albinana-Gimenez, N., Clemente-Casares, P., Bofill-Mas, S., Hundesa, A., Ribas, F., \& Girones, R. (2006). Distribution of Human Polyomaviruses, Adenoviruses, and Hepatitis E Virus in the Environment and in a Drinking-Water Treatment Plant. Environmental Science Technology, 40, 7416-7422

Assembly of First Nations (AFN). (2008a). Letter from Richard Jock, AFN to Karl Carisse, INAC Regarding Serious Objections to the Unilateral Approach to Consultation and the Pursuit on the Single Option Regarding the Proposed Legislation for Application to First Nations communities. Retrieved from http://www.afn.ca/uploads/files/water/08-08- 2.pdf.

Association of Municipalities Ontario. (2012). Municipalities in Ontario. Retrieved from http://www.amo.on.ca/YLG/ylg/muniont.html.

Auclair, N., \& Simeone, T. (2010). Legislative summary: Bill S-11: The safe drinking water for First Nations Act. Publication No. 40-3-S1 I-E. Ottawa, ON: Library of Parliament. Retrieved from: 
http://www2.parl.gc.ca/Sites/LOP/LegislativeSummaries/Bills_ls.asp?lang=E\&ls $=$ s1 $1 \&$ source $=$ library prb\&Parl $=40 \&$ Ses $=3$

Blatchford, C. (2010). A case study on First Nations poverty. Globe and Mail, December 30. Retrieved from http://media.knet.ca/node/10939.

Canadian Council of Ministers of the Environment (CCME). (2002). From source to tap: Guidance on the multi-barrier approach to safe drinking water. Initial report by the Federal-Provincial-Territorial Committee on Drinking Water and the CCME Water Quality Task Group.

CCME. (2004). From source to tap: Guidance on the multi-barrier approach to safe drinking water. Final report by the Federal-Provincial-Territorial Committee on Drinking Water and the CCME Water Quality Task Group. Retrieved from: http://www.ccme.ca/assets/pdf/mba_guidance_doc_e.pdf.

Canadian Encyclopedia. (2012). Greater Sudbury. Retrieved from http://www.thecanadianencyclopedia.com/articles/greater-sudbury.

Canadian Medical Association. (2008). Investigative report: 1776 boil-water advisories now in place in Canada. Canadian Medical Association Journal, 178, 1261-1263.

Chen, H.T. (1990). Intervening Mechanism Evaluation. In Theory Driven Evaluations. Thousand Oaks, CA: Sage Publications Inc.

Chen, H.T. (2005). Practical Program Evaluation: Assessing and Improving Planning, Implementation, and Effectiveness. Thousand Oaks, CA: Sage Publications Inc.

Chiefs of Ontario (CoO). (2005). Drinking Water in First Nations Communities. Retrieved from http://chiefs-ofontario.org/PageContent/Default.aspx?SectionHeadlineID=98.

CoO. (2008a). COO Bulletin Regarding Indian and Northern Affairs Canada (INAC) Proposed Legislation on Water and Wastewater. Retrieved from http://www.afn.ca/uploads/files/water/08-08-18.pdf.

CoO. (2008b). COO Water Declaration of the First Nations in Ontario. Retrieved from http://www.afn.ca/uploads/files/water/08-10-00.pdf

CoO. (2009). Letter from COO to Minister Strahl Regarding INAC's Proposed Legislative Framework for Drinking Water and Wastewater in First Nations Communities. Retrieved from http://www.afn.ca/uploads/files/water/09-11-05.pdf.

CoO. (2010). COO Resolution Bill S-11 An Act Respecting the Safety of Drinking Water on First Nations. Retrieved from http://www.afn.ca/uploads/files/water/10-1123.pdf.

CoO. (2012a). Home. Retrieved from http://www.chiefs-of-ontario.org/. 
CoO. (2012b). About Us. Retrieved from http://chiefs-ofontario.org/PageContent/Default.aspx?SectionID=6\&SectionHeadlineID=58

Conboy, M. J., \& Goss, M. J. (2007). Contamination of rural drinking water wells by fecal origin bacteria. Water Quality Research Journal of Canada, 34, 281-303.

Cool, G. et al. (2011). Evaluation of the vulnerability to contamination of drinking water systems for rural regions in Québec, Canada. Journal of Environmental Planning and Management, 53, 615-638.

Cool, G., Rodriguez, M. J., Bouchard, C., Levallois, P., \& Joerin, F. (2010). Evaluation of the vulnerability to contamination of drinking water systems for rural regions in Québec, Canada. Journal of Environmental Planning and Management, 53, 615-638.

Coulibaly, H.D. \& Rodriguez, M.J. (2003). Spatial and temporal variation of drinking water quality in ten small Quebec utilities. Journal of Environmental Engineering Science, 2, 47-61.

Davies, J. M., \& Mazumder, A. (2003). Health and environmental policy issues in Canada: the role of watershed management in sustaining clean drinking water quality at surface sources. Journal of Environmental Management 68, 273-286.

Delgamuukw v. British Columbia, 3 S.C.R. 1010. (1997). Retrieved from http://www.canlii.org/en/ca/scc/doc/1997/1997canlii302/1997canlii302.pdf.

DWSP (Ontario Drinking Water Surveillance Program). (1997). Executive summaries: Report for 1993, 1994 and 1995 drinking water surveillance program. Retrieved from http://www.ene.gov.on.ca/stdprodconsume/groups/lr/@ene/@resources/document s/resource/std01_079686.pdf.

DWSP (Ontario Drinking Water Surveillance Program). (2011). Map of the DWSP Monitoring Network. Retrieved from http://www.ene.gov.on.ca/stdprodconsume/groups/lr/@ene/@monitoring/docume nts/nativedocs/stdprod_078990.pdf.

Eggerston, L. (2008). Despite federal promises, First Nations' water problems persist. Canadian Medical Association Journal, 178, 985.

Environment Canada (EC). (1992). Clean Water - Life Depends on It! Retrieved from http://www.ec.gc.ca/eau-water/default.asp?lang $=E n \& n=06 \mathrm{~F} 1403 \mathrm{C}-1$

EC. (2001). Water, Art, and the Canadian Identity: At the Water's Edge. Retrieved from http://www.ec.gc.ca/eau-water/default.asp?lang $=$ en\&n=62D086E9-1

EC. (2003). Water and Canada: Preserving a Legacy for People and the Environment. Retrieved from http://www.ec.gc.ca/Publications/AFF1D423-17C8-4E20-BA0F- 
91773EF7356F\%5CWater-and-Canada-Preserving-a-Legacy-for-People-and-theEnvironment.pdf

EC. (2006a). Water - How good is it? Retrieved from http://www.ec.gc.ca/eauwater/default.asp?lang=En\&n=1 A0A0DC6-1

EC. (2006b). Water - How We Manage It. Retrieved from http://www.ec.gc.ca/eauwater/default.asp?lang=En\&n=3DC41 CC $0-1$

EC. (201 la). Water Quality Monitoring. Retrieved from http://www.ec.gc.ca/qualitedeleauwaterquality/default.asp?lang=En\&n=0DF8E8D2-1.

EC. (2011b). Federal Policy and Legislation. Retrieved from http://www.ec.gc.ca/eauwater/default.asp?lang=En\&n=E05A7F81-1.

EC. (2011c). 2011 Municipal Water Pricing Report: Municipal Water Pricing 2009

Statistics. Retrieved from http://www.ec.gc.ca/Publications/992156D4-25994026-9B4C.

47855D26CCB8/2011MunicipalWaterPricingReport2009Statistics.pdf.

EC. (2012a). Groundwater Contamination. Retrieved from http://www.ec.gc.ca/eauwater/default.asp?lang $=$ En\&n=6A7FB7B2-1

EC. (2012b). About Environment Canada. Retrieved from http://www.ec.gc.ca/default.asp?lang=En\&n=BD3CE17D-1.

EC. (2012c). Shared Responsibility. Retrieved from http://www.ec.gc.ca/eauwater/default.asp?lang=En\&n=035F6173-1.

EC. (2012d). Wise Water Use. Retrieved from http://www.ec.gc.ca/eauwater/default.asp?lang=En\&n=F25C70EC-1.

Fox, A. (2002). Pikangikum wins landmark court decision: Judge rules Nault breached 'the duty of procedural fairness'. Wawatay News, 29, n.p.

Ginoogaming First Nation. (2011). About us. Retrieved from http://www.ginoogaming.ca/?pgid=2

Ginoogaming. (2012a). Chief and Council. Retrieved from http://www.ginoogaming.ca/?pgid=4.

Ginoogaming. (2012b). Band Office Staff. Retrieved from $\mathrm{http}: / / \mathrm{www}$.ginoogaming.ca/?pgid=5.

Ginoogaming. (2012c). Water and Sewer. Retrieved from http://www.ginoogaming.ca/?pgid=37. 
Goss, M.J., Barry, D.A.J. \& Rudolph, D.L. (1998). Groundwater contamination in Ontario farm wells and its association with agriculture. Journal of Contamination Hydrology, 32, 267-293.

Government of Canada (GOC). (1985). Fisheries Act, 1985. Retrieved from http://lawslois.justice.gc.ca/eng/acts/F-14/.

GOC. (2010). A stronger Canada. A stronger economy. Now and for the future. Speech from the Throne. Retrieved from: http://www.speech.gc.ca/grfx/docs/sft-ddt2010_e.pdf.

GOC. (2012a). Jobs Growth and Long-Term Prosperity: Economic Action Plan 2012. Retrieved from http://www.budget.gc.ca/2012/plan/pdf/Plan2012-eng.pdf.

GOC. (2012b). Harper Government Announces Plan for Responsible Resource Development. Retrieved from http://news.gc.ca/web/articleeng.do? $\mathrm{m}=\% 2$ Findex \&nid $=669169$

Graham, J. (2002). Safe Water for First Nation Communities: Learning the Lessons from Walkerton. Conference Paper: First International Conference on Water.

Graham, J. (2003). Safe Water for First Nations: Charting a Course for Reform. Policy Brief No.14 January.

Graham, J. \& Fortier, E. (2006). Building Governance Capacity: the Case of Potable Water in First Nations Communities. Conference Paper: The Aboriginal Policy Research Conference, March 23, Ottawa.

Greater Sudbury. (2004). 2004 Section 11 Annual Report: Dowling Drinking Water System. Retrieved from http://www.greatersudbury.ca/waterworks/annualPDF2004/AR_fnl_2004_large/d owling2004.pdf.

Greater Sudbury. (2005). 2005 Section 11 Annual Report: Dowling Drinking Water System. Retrieved from http://www.greatersudbury.ca/waterworks/annualPDF2005/dowling\%202005\%20 annual\%20report.pdf.

Greater Sudbury. (2006). 2006 Section 11 Annual Report: Dowling Drinking Water System. Retrieved from http://www.greatersudbury.ca/waterworks/annualPDF2006/dowling\%202006\%20 annual\%20report.pdf.

Greater Sudbury. (2007). 2007 Section 11 Annual Report: Dowling Drinking Water System. Retrieved from http://www.greatersudbury.ca/waterworks/annualPDF2007/dowling_2007.pdf. 
Greater Sudbury. (2008). 2008 Section 11 Annual Report: Dowling Drinking Water System. Retrieved from http://www.greatersudbury.ca/content/div_water/documents/dowling\%202008r4.pdf.

Greater Sudbury. (2009). 2009 Section 11 Annual Report: Dowling Drinking Water System. Retrieved from http://www.greatersudbury.ca/content/div_water/documents/Dowling\%20$\% 20 \mathrm{R} 4 \% 20-\% 202009$.pdf.

Greater Sudbury. (2010a). Part Ten: Part Ten Dowling Drinking Water System. In Greater Sudbury Source Protection Area Proposed Assessment Report. Greater Sudbury, ON.

Greater Sudbury. (2010b). 2010 Section 11 Annual Report: Dowling Drinking Water System. Retrieved from http://www.greatersudbury.ca/content/div_water/documents/Dowling\%202010.pd f.

Greater Sudbury. (2011a). 2011 Water Works Summary Report: Large Municipal Residential Systems. Retrieved from http://www.greatersudbury.ca/content/div_water/documents/2011\%20Summary\% 20Report.pdf.

Greater Sudbury. (2011b). 2011 Section 11 Annual Report: Dowling Drinking Water System. Retrieved from http://www.greatersudbury.ca/content/div_water/documents/2011\%20Annual\%20 Water\%20Quality\%20Report1.pdf.

Greater Sudbury. (2012a). About Greater Sudbury. Retrieved from http://www.sudburytourism.ca/index.php?option=com_content\&view=article\&id $=114 \&$ Itemid $=165 \&$ lang $=$ en.

Greater Sudbury. (2012b). Town of Onaping Falls. Retrieved from http://www.sudburymuseums.ca/index.cfm?app=w_vmuseum\&lang=en\&currID= 1398\&parID=1390.

Greater Sudbury. (2012c). 12 Ward Model. Retrieved from http://www.greatersudbury.ca/cms/index.cfm?app=div_council\&lang=en\&currID $=7060$.

Greater Sudbury. (2012d). Departments Contacts: Infrastructure. Retrieved from http://www.city.greatersudbury.on.ca/cms/index.cfm?app=dept_ies\&lang=en\&cur rid $=3039$.

Greater Sudbury. (2012e). Organizational Chart-February $16^{\text {th }} 2012,4 A 1-1$. 
Greater Sudbury. (2012f). Services. Retrieved from

http://www.city.greatersudbury.on.ca/cms/index.cfm?app=dept_ies\&lang=en\&cur rid $=3038$.

Greater Sudbury. (2012g). Quality Management System Policy. Retrieved from http://www.greatersudbury.ca/cms/index.cfm?app=div_water\&lang=en\&currID= 8789.

Greater Sudbury. (2012h). Dowling Tank Inspections Schedule.

Greenstone. (2012a). The Municipality of Greenstone. Retrieved from http://www.greenstone.ca/MunicipalMatters.aspx.

Greenstone. (2012b). Greenstone Municipal Council 2010 - 2014. Retrieved from http://www.greenstone.ca/MunicipalMatters/Council.aspx.

Greenstone. (2012c). Municipality of Greenstone Public Services. Retrieved from http://www.greenstone.ca/ResidentialLife/PublicServices.aspx.

Greenstone. (2012d). Contact Us. Retrieved from http://www.greenstone.ca/MunicipalMatters/Contacts.aspx.

Greenstone. (2012e). The Corporation of the Municipality of Greenstone: By-Law 12-25. Retrieved from http://greenstone.ca/DesktopModules/Bring2mind/DMX/Download.aspx?TabID= 147\&EntryId=5415\&Command=Core_Download\&PortalId=0\&TabId=147.

Harden, A. \& Levalliant, H. (2008). Boiling Point! Six community profiles of the water crisis facing First Nations within Canada. Ottawa: Polaris Institute.

Health Canada (HC). (2009). Drinking Water Advisories in First Nations Communities in Canada - A National Overview 1995-2007. Retrieved from http://www.hcsc.gc.ca/fniah-spnia/pubs/promotion/_environ/2009_water-qualit-eaucanada/index-eng.php.

HC. (2010a). Guidelines for Canadian Drinking Water Quality. Retrieved from http://www.hc-sc.gc.ca/ewh-semt/water-eau/drink-potab/guide/index-eng.php.

HC. (2010b). Ensuring Safe Drinking Water in First Nations Communities in Canada. Retrieved from http://www.hc-sc.gc.ca/fniah-spnia/promotion/publicpublique/sfw-sep-eng.php.

HC. (2010c). Guidelines for Canadian Drinking Water Quality - Summary Table. Retrieved from http://www.hc-sc.gc.ca/ewh-semt/alt_formats/hecs-

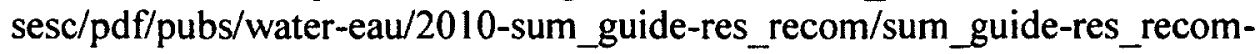
eng.pdf. 
HC. (2011a). Drinking water and wastewater. Retrieved from http://www.hcsc.gc.ca/fniah-spnia/promotion/public-publique/water-eau-eng.php.

HC. (2011b). Procedural Guidelines for Waterborne Disease Events in First Nations Communities South of $60^{\circ}$. Retrieved from http://www.hc-sc.gc.ca/fniahspnia/pubs/promotion/_environ/south-sud-60/index-eng.php.

HC. (2012a). About Mission, Values, Activities. Retrieved from http://www.hcsc.gc.ca/ahc-asc/activit/about-apropos/index-eng.php.

HC. (2012). About Health Canada. Retrieved from http://www.hc-sc.gc.ca/ahcasc/index-eng.php.

HC. (2012b). First Nations \& Inuit Health. Retrieved from http://www.hc-sc.gc.ca/fniahspnia/index-eng.php.

Highway 11. (2011). Longlac. Retrieved from http://www.highwayl1.ca/ThunderBay/01Longlac/index.php.

Hill, C., Furlong, K., Bakker, K., \& Cohen, A. (2008). Harmonization versus subsidiarity in water governance: A review of water governance and legislation in the provinces and territories. Canadian Water Resources Journal, 33, 315-332.

Holme, R. (2003). Drinking water contamination in Walkerton, Ontario: Positive resolutions from a tragic event. Water Science and Technology, 47, 1-6

Hrudey, S.E. (2008). Safe drinking water? It depends on where you live! Canadian Medical Association Journal, 178, 975.

Hrudey, S.E., Huck, P.M., Payment, P., Gillham, R.W., \& Hrudey, E.J. (2002). Walkerton: Lessons learned in comparison with waterborne outbreaks in the developed world Journal of Environmental Engineering Science, 1, 397-407.

Hrudey, S.E., Payment, P., Huck, P.M., Gillham, R.W., \& Hrudey, E.J. (2003). A fatal waterborne disease epidemic in Walkerton, Ontario: comparison with other waterborne outbreaks in the developed world. Water Science and Technology, 47, $7-14$

Hurley, M.C. (2000). Aboriginal Title: The Supreme Court of Canada Decision in Delgamuukw v. British Columbia. Publication No. BP-459E. Ottawa, ON: Library of Parliament. Retrieved from: http://www.parl.gc.ca/Content/LOP/ResearchPublications/bp459-e.htm.

Independent First Nations Alliance (IFNA). (2000). Assessment study of water and wastewater systems and associated water management practices in Ontario First Nation communities: Pilkangikum First Nation (Band No. 208). Retrieved from: http://www.cbc.ca/slowboil/pdfs/on/Pikangikum.pdf. 
IFNA (2012). Pikangikum First Nation Community Profile. Retrieved from http://www.ifna.ca/article/pikangikum-116.asp.

Indian and Northern Affairs Canada (INAC). (2006). Protocol for Safe Drinking Water in First Nations Communities. Retrieved from http://www.aadncaandc.gc.ca/DAM/DAM-INTER-HQ/STAGING/textetext/sdw_1100100015850_eng.pdf.

INAC. (2007). Canada's New Government Invests More Than \$46 Million in Infrastructure at Pikangikum First Nation. Retrieved from http://www.aadncaandc.gc.ca/aiarch/mr/nr/j-a2007/2-2870-eng.asp

INAC. (2008). Government Of Canada Announces Next Important Steps To Improve Drinking Water In First Nations Communities. Retrieved from http://www.aadncaandc.gc.ca/aiarch/mr/nr/j-a2008/2-3019-eng.asp

INAC. (2010a). INAC's Support for First Nations Water and Wastewater Infrastructure April 1, 2006 - March 31, 2010. Retrieved from http://www.aadncaandc.gc.ca/eng/1313696742768.

INAC. (2010b) Protocol for Centralised Drinking Water Systems in First Nations Communities: Protocol for Centralised Drinking Water Systems in First Nations Communities. Retrieved from http://www.aadnc-aandc.gc.ca/eng/l100100034998.

INAC. (2010c) Protocol for Decentralised Drinking Water Systems in First Nations Communities: Protocol for Centralised Drinking Water Systems in First Nations Communities. Retrieved from http://www.aadnc-aandc.gc.ca/eng/1100100034998.

INAC. (2010d). INAC's Support for First Nations Water and Wastewater Infrastructure April 1, 2006 - March 31, 2010. Retrieved from http://www.aadncaandc.gc.ca/eng/1313696742768.

Indian and Northern Affairs Canada \& Health Canada (INAC \& HC). (2007). Summative Evaluation of the First Nations Water Management Strategy. Retrieved from http://www.aincinac.gc.ca/ai/arp/aev/pubs/ev/efnw08/efnw08-eng.asp

Isfeld, H. (2009). Boil water advisory mapping project: An exploration and review of available data. Prairie Women's Health Centre for Excellence, Project 193.

Khan, F., Husain, T., \& Lumb, A. (2003). Water quality evaluation and trend analysis in selected watersheds of the Atlantic region of Canada. Environmental Monitoring and Assessment 88, 221-242.

KPMG. (2011) City of Greater Sudbury Financial Plan for Water and Wastewater Services: Prepared Pursuant to Ontario Regulation 453/07 - Final Report March 1st, 2011. Retrieved from http://www.greatersudbury.ca/content/div_water/documents/City_Water_and_Wa stewater_Financial_Plan-Mar.pdf. 
Kneeshaw, D. (2006). Kamloops Indian Band. Retrieved from: http://www.epssdw.gc.ca/inlv/sbms_e.asp?uDsclm=1.

Lui, E. (2011). Tenth Session of the United Nations Permanent Forum on Indigenous Issues: Panel on the Right to Water: Submission of the Council of Canadians.

Mackenzie, C.A., Lockridge, A., \& Keith, M. (2005). Declining Sex Ratio in a First Nation Community. Environmental Health Perspectives, 113, 1295-1298.

MacKinnon, M. (2005). A First Nations Voice in the Present Creates Healing in the Future. Canadian Journal of Public Health, 91, S13-16.

Magnet, J.E. (2001). Memorandum and Fact of Law: T-785-01. Retrieved from http://www.constitutional-law.net/pikangik.pdf.

Manta. (2012). Northern Waterworks Inc. Retrieved from http://www.manta.com/ic/mtqyx8f/ca/northern-waterworks-inc.

Mascarenhas, M. (2007a). Where the waters divide: First Nations, tainted water and environmental justice in Canada. Local Environment, 12, 565-577.

Mascarenhas, M. (2007b). Where the waters divide: Environmental justice, neoliberalism, and Aboriginal voices. An ethnography of the changing Canadian water sector [Dissertation]. Michigan State University.

Mayberry, R. (2010). National Assessment of First Nations Water and Wastewater Systems: Pikangikum Site Visit Report - Final.

McDiarmid, M. (2012). Budget bill makes complex changes to fisheries protection: Greens leader Elizabeth May fears legislation will eliminate habitat protection. Retrieved from http://www.cbc.ca/news/politics/story/2012/05/03/pol-budget-billfisheries.html

Ministry of Infrastructure (MOI). (2012a). Ontario Small Waterworks Assistance Program. Retrieved from http://www.moi.gov.on.ca/en/infrastructure/sectors/oswap.asp.

MOI. (2012b). Ontario Small Waterworks Assistance Program - Phase 3 Intake 1 Successful Applicants. Retrieved from http://www.moi.gov.on.ca/en/news/bg201 10518.asp.

Ministry of Municipal Affairs and Housing (MAH). (2012a).Municipal Restructuring. Retrieved from http://www.mah.gov.on.ca/Page247.aspx.

MAH. (2012b). Municipal Restructuring Activity Summary Table. Retrieved from http://www.mah.gov.on.ca/AssetFactory. aspx?did=6212 
Ministry of Northern Development and Mines (MNDM). (2012). Northern Ontario: A Profile. Retrieved from http://www.mndm.gov.on.ca/northern_development/documents/northern_ontario_ e.pdf.

Ministry of the Environment (MOE). (2008). Chapter 3: Section 3.12: Ontario Clean Water Agency. In MOE 2008 Annual Report of the Office of the Auditor General of Ontario.

MOE. (201 1a). Annual Report 2009-2010: Chief Drinking Water Inspector. Retrieved from

http://www.ene.gov.on.ca/stdprodconsume/groups/lr/@ene/@resources/document s/resource/stdprod_083949.pdf.

MOE. (201 1b). Minister's Annual Report on Drinking Water. Retrieved from http://www.ene.gov.on.ca/stdprodconsume/groups/lr/@ene/@resources/document s/resource/stdprod_088400.pdf.

MOE. (2012a). The Dowling Drinking Water System: Drinking Water System Inspection Report.

MOE. (2012b). Dowling Drinking Water System. Retrieved from $\mathrm{http}$ ://www.ene.gov.on.ca/environment/dwo/en/mapping/report/system?dws=2100 01665 .

MOE. (2012c). Red Lake Drinking Water System: Drinking Water System Inspection Report.

MOE. (2012d). Red Lake Drinking Water System. Retrieved from $\mathrm{http} / / \mathrm{www}$.ene.gov.on.ca/environment/dwo/en/mapping/report/system?dws=2100 00265

MOE. (2012e). The Town of Longlac Water Treatment System: Drinking Water System Inspection Report.

MOE. (2012f). Drinking Water System Information: Longlac Drinking Water System. Retrieved from http://www.ontario.ca/ONT/portal61/drinkingwater/DWPPlant?DWPQualityActi on=displayPlant\&plant $=220000264 \&$ lang $=e n$.

MOI. (2012b). Ontario Small Waterworks Assistance Program - Phase 3 Intake 1 Successful Applicants. Retrieved from http://www.moi.gov.on.ca/en/news/bg201 10518.asp.

Mullen, J., Vladi, N., \& Mills, A. J. (2006). Making sense of the Walkerton crisis. Culture and Organization, 12, 207-220. 
Murdocca, C. (2010). "There Is Something in That Water": Race, Nationalism, and Legal Violence. Law \& Social Inquiry, 35, 369-402.

Neapetung, R. (2006). Yellow Quill First Nation: A case study. Retrieved from: http://www.eps-sdw.gc.ca/inlv/sbms_e.asp?uDsclm=1.

Neegan Burnside. (201 la). National Assessment of First Nations Water and Wastewater Systems - British Columbia Regional Roll-Up Report. Retrieved from http://www.aadnc-aandc.gc.ca/DAM/DAM-INTER-HQ/STAGING/textetext/enr_wtr_nawws_rurbc_rurbc_1315622900946_eng.pdf.

Neegan Burnside. (201 lb). National Assessment of First Nations Water and Wastewater Systems - National Roll-Up Report. Retrieved from http://www.aadncaandc.gc.ca/eng/1313770257504

Neegan Burnside. (2011 c). National Assessment of First Nations Water and Wastewater Systems - Ontario Regional Roll-Up Report. Retrieved from

Neegan Burnside. (2011d). National Assessment of First Nations Water and Wastewater Systems - Atlantic Regional Roll-Up Report. Retrieved from http:/www.aadncaandc.gc.ca/eng/1314206899796/1314207059703

Neegan Burnside. (2011e). National Assessment of First Nations Water and Wastewater Systems - Quebec Regional Roll-Up Report. Retrieved from http://www.aadncaandc.gc.ca/eng/1314372684986/1314372772921

Neegan Burnside. (2011 f). National Assessment of First Nations Water and Wastewater Systems - Manitoba Regional Roll-Up Report. Retrieved from http://www.aadncaandc.gc.ca/eng/1315322645420/1315322706937

Neegan Burnside. (2011 g). National Assessment of First Nations Water and Wastewater Systems - Saskatchewan Regional Roll-Up Report. Retrieved from http://www.aadnc-aandc.gc.ca/eng/1315530095418/1315530190074

Neegan Burnside. $(2011 \mathrm{~h})$. National Assessment of First Nations Water and Wastewater Systems - Alberta Regional Roll-Up Report. Retrieved from http://www.aadncaandc.gc.ca/eng/1315502688073/1315502878844

Northern Waterworks. (2008). Red Lake Drinking Water System: Annual Report. Retrieved from http://www.red-lake.com/pdf/other/2008wtpreports.pdf.

Northern Waterworks. (2010). Red Lake Drinking Water System: Annual Report. Retrieved from http://www.red-lake.com/pdf/other/2008wtpreports.pdf.

Northern Waterworks. (2011). Red Lake Drinking Water System: Annual Report. Retrieved from http://www.redlake.ca/pdf/water/2011WTP_ANNUALREPORT.pdf. 
Northern Waterworks (2012a). Working with Municipal Government. Retrieved from http://www.northernwaterworks.com/html/municipal.html.

Northern Waterworks (2012b). About Northern Waterworks. Retrieved from http://www.northernwaterworks.com/html/about_us.html.

Northern Waterworks. (2012c). Company Directory. Retrieved from http://www.northernwaterworks.com/html/contact_us.html.

Northern Waterworks (2012d). Working with First Nations. Retrieved from http://www.northernwaterworks.com/html/first_nations.html.

Northwestern Health Unit (NWHU). (2006). Inspection Report on the Pikangikum Water and Sewage Systems. Retrieved from http://www.turtleisland.org/healing/pikangikum06a.pdf.

O'Connor, D.R. (2002a). Part I: Report of the Walkerton Inquiry: The Events of May 2000. Toronto, ON: Ontario Ministry of the Attorney General.

O'Connor, D.R. (2002b). Part II: Report of the Walkerton Inquiry: A Strategy for Safe Drinking Water. Toronto, ON: Ontario Ministry of the Attorney General.

Ontario. (2002). Safe Drinking Water Act, 2002. Retrieved from http://www.elaws.gov.on.ca/html/statutes/english/elaws_statutes_02s32_e.htm.

Ontario Clean Water Association (OCWA). (2002). North Shore Tribal Council: First Nation Report Phase II. Retrieved from

http://www.cbc.ca/slowboil/pdfs/on/North\%20Shore\%20Tribal\%20Council\%202. pdf.

OCWA. (2011). 2010 Section 11 Annual Report: Longlac Drinking-Water System. Retrieved from http://www.greenstone.ca/ResidentialLife/PublicServices/WaterSewerInformation .aspx.

OCWA. (2012a). 2011 Section 11 Annual Report: Longlac Drinking-Water System. Retrieved from http://www.greenstone.ca/LinkClick.aspx?fileticket=1 Bwzp42Tgeg\%3d\&tabid=1 545.

OCWA. (2012b). 2011 Schedule 22 Annual Summary Report: Longlac Drinking-Water System. Retrieved from http://www.greenstone.ca/LinkClick.aspx?fileticket=talk0HzYw0o\%3d\&tabid=1 545.

OCWA. (2012c). Company Profile. Retrieved from http://www.ocwa.com/en/company_profile. 
Peterson, H. (n.d.). Rural drinking water and waterborne illness. Retrieved from http://www.safewater.org/PDFS/scientificresearch/Rural_Drink_Waterborne_Ill.p df.

Peterson, H., \& Torchia, M. (2008). Letter Written by the Executive Director of the Safe Drinking Water Foundation, Dr. Hans Peterson, to the editor of the Canadian Medical Journal Association.

Paznar, M.J. (2011). National Assessment of First Nations Water and Wastewater Systems: Sagamok Anishnawbek First Nation Site Visit Report - Final. Orangeville, ON: Neegan Burnside LTD.

Profile Canada. (2012). Northern Waterworks Inc. Retrieved from http://www.profilecanada.com/companydetail.cfm?company=2491911_Northern_ Waterworks_Inc_Red_Lake_ON.

Red Lake (2005). By-Law No. 554-05. Retrieved from http://www.redlake.ca/pdf/bylaw/bylaw554-05.pdf

Red Lake. (2010). Red Lake drinking water system annual report. Retrieved from http://www.redlake.ca/pdf/water/2010water_reports.pdf.

Red Lake. (2012a). Red Lake - Gateway to Woodland Caribou Provincial Park. Retrieved from http://www.redlake.ca/cms/.

Red Lake. (2012b). Mayor and Council. Retrieved from http://www.redlake.ca/cms/index.php?option=com_contact\&view=category\&cati $\mathrm{d}=55$ \&Itemid $=71$.

Red Lake. (2012c). Municipal Contacts. Retrieved from http://www.redlake.ca/cms/index.php?option=com_contact\&view=category\&cati $\mathrm{d}=56$ \& Itemid $=204$.

Red Lake. (2012d). Schedule K-2 to By-Law 1563-11. Retrieved from http://www.redlake.ca/pdf/bylaw/fees/k2.pdf.

Red Lake. (2012e). Capital Budget: 2011. Retrieved from http://www.redlake.ca/pdf/budget/201 lbc.pdf.

Rudolph, D.L., Barry, D.A.J., \& Goss, M.J. (1998). Contamination in Ontario farmstead domestic wells and its association with agriculture. Journal of Contamination Hydrology, 32, 91-107.

Rudolph, D. \& Goss, M.J. (1993). The Ontario farm groundwater quality survey summer 1992. Report to Agriculture Canada under the Federal-Provincial Environmental Sustainability Initiative, June, Guelph, Ont. 
Sagamok. (2006). Chief \& Council. Retrieved from http://sagamok.firstnation.ca/?q=Council.

Sagamok. (2012a). Chief and Council. Retrieved from http://www.sagamok.ca/chiefcouncil.

Sagamok. (2012b). Education. Retrieved from http://www.sagamok.ca/programsservices/education.

Sagamok. (2012c). Programs \& Services. Retrieved from http://www.sagamok.ca/programs-services.

Sagamok. (2012d). Lands, Resources and Infrastructure. Retrieved from http://www.sagamok.ca/programs-services/lands-resources-infrastructure.

Sagamok. (2012e). Job Opportunities. Retrieved from http://www.sagamok.ca/community/job-opportunities.

Saint Germain, J. et al. (2007). Safe drinking water for First Nations: The final report of the standing senate committee on Aboriginal peoples. Retrieved from: http://www.parl.gc.ca/39/1/parlbus/commbus/senate/com-e/abor-e/repe/rep08jun07-e.htm.

Salma, P. (2010). National Assessment of First Nations Water and Wastewater Systems: Ginoogaming First Nation Site Visit Report-Final. Orangeville ON: Neegan Burnside LTD.

Schuster, C.J., et al. (2005). Infectious disease outbreaks related to drinking water in Canada, 1974-2001. Canadian Journal of Public Health, 96, 254-259.Snider, L. (2004). Resisting neo-liberalism: The poisoned water disaster in Walkerton, Ontario. Social \& Legal Studies, 13, 265-289.

SDWF. (2011). Current Projects. Retrieved from http://www.safewater.org/aboutus/current-projects.html.

Smith, D.W., Guest, R.K., Svrcek, C.P., \& Farahbakhsh, K. (2006). Public health evaluation of drinking water systems for First Nations reserves in Alberta, Canada. Journal of Environmental Engineering Science, 5, S1-S17.

Statistics Canada. (2006). Community profiles: Thessalon. http://www12.statcan.gc.ca/census-recensement/2006/dp-pd/prof/92$591 /$ details/Page.cfm?Lang $=$ E \&Geol $=$ CSD\&Code $1=3557026 \& G e 02=P R \&$ Code $2=35 \&$ Data $=$ Count $\&$ Search Text $=$ Thessalon $\% 2012 \&$ Search Type=Begins $\&$ Search $\mathrm{PR}=01 \& \mathrm{~B} 1=\mathrm{All} \&$ Custom $=$.

Statistics Canada. (2012a). Sagamok, Ontario (Code 3557071) and Algoma, Ontario (Code 3557) (table). Census Profile. 2011 Census. Statistics Canada Catalogue 
no. 98-316-XWE. Ottawa. Retrieved from http://wwwl2.statcan.ca/censusrecensement/2011/dp-pd/prof/index.cfm?Lang=E.

Statistics Canada. (2012b). Dowling, Ontario (Code 1084) and Ontario (Code 35) (table). Census Profile. 2011 Census. Statistics Canada Catalogue no. 98-316XWE. Ottawa. Retrieved from http://wwwl2.statcan.ca/censusrecensement $2011 / \mathrm{dp}$-pd/prof/index.cfm?Lang=E.

Statistics Canada. (2012c). Greater Sudbury / Grand Sudbury, Ontario (Code 580) and Ontario (Code 35) (table). Census Profile. 2011 Census. Statistics Canada Catalogue no. 98-316-XWE. Ottawa. Retrieved from http://www12.statcan.ca/census-recensement/2011/dp$\mathrm{pd} /$ prof/index.cfm?Lang=E.

Statistics Canada. (2012d). Statistics Canada. (2006). Community profiles: Red Lake. Retrieved from http://wwwl2.statcan.gc.ca/census-recensement/2006/dppd/prof/92591/details/Page.cfm?Lang=E\&Geo1=CSD\&Code $1=3560042 \& G e o 2=P R \&$ Code $2=35 \&$ Data $=$ Count $\&$ Search Text $=$ red\%20lake $\&$ Search Type $=$ Begins $\&$ SearchPR $=$ $01 \& \mathrm{~B} 1=\mathrm{All} \&$ Custom $=$

Statistics Canada. (2012e). Pikangikum 14, Ontario (Code 3560077) and Ontario (Code 35) (table). Census Profile. 2011 Census. Statistics Canada Catalogue no. 98-316XWE. Ottawa. Retrieved from http://www12.statcan.gc.ca/censusrecensement/2011/dp-pd/prof/index.cfm?Lang=E.

Statistics Canada. (2012f). Red Lake, Ontario (Code 3560042) and Kenora, Ontario (Code 3560) (table). Census Profile. 2011 Census. Statistics Canada Catalogue no. 98-316-XWE. Ottawa. Retrieved from http://wwwl2.statcan.gc.ca/censusrecensement/2011/dp-pd/prof/index.cfm?Lang=E.

Statistics Canada. (2012g). Longlac, Ontario (Code 0481) and Ontario (Code 35) (table). Census Profile. 2011 Census. Statistics Canada Catalogue no. 98-316-XWE. Ottawa. Retrieved from http://www12.statcan.ca/census-recensement/2011/dp$\mathrm{pd} /$ prof/index.cfm?Lang=E.

Statistics Canada. (2012h). Ginoogaming First Nation, Ontario (Code 3558067) (table). Census Profile. 2011 Census. Statistics Canada Catalogue no. 98-316-XWE. Ottawa. Retrieved from http://wwwl2.statcan.ca/census-recensement/2011/dp$\mathrm{pd} / \mathrm{prof} /$ index.cfm?Lang=E.

Statistics Canada. (2012i). Greenstone, Ontario (Code 3558075) and Thunder Bay, Ontario (Code 3558) (table). Census Profile. 2011 Census. Statistics Canada Catalogue no. 98-316-XWE. Ottawa. Retrieved from http://wwwl2.statcan.ca/census-recensement/2011/dp$\mathrm{pd} /$ prof/index.cfm?Lang=E. 
Swain, H., Louttit, S., \& Hrudey, S. (2006). Report of the expert panel on safe drinking water for First Nations: Part I. Retrieved from: http://www.sdweps.gc.ca/rsrsc/volume_1_e.pdf.

Wallis, P.M., Erlandsen, S.L., Isaac-Renton, J.L., Olson, M.E., Robertson, W.J., \& Van Keulen, H. (1996). Prevalence of Giardia Cysts and Cryptosporidium Oocysts and Characterization of Giardia spp. Isolated from Drinking Water in Canada. Applied and Environmental Microbiology, 62, 2789-2797.

Whitefeather Forest Initiative (WFI). (2012). Our First Nation. Retrieved from http://www.whitefeatherforest.com/our-first-nation/.

Willms \& Shier Environmental Lawyers. (2006). Report of the expert panel on safe drinking water for First Nations: Part II. Retrieved from: http://www.sdweps.gc.ca/rsrsc/volume_2_e.pdf.

Wilson, P. (2004). First Nations Integrated Watershed Management. In D. Shrubsole (ED) Canadian perspectives on integrated resource management. Cambridge: Canadian Water Resources Association. 


\section{Glossary of Terms}

Capacity Gap: The difference between First Nations current capabilities (including training, knowledge, infrastructure, and financial and human resources) to provide quality drinking water to their communities and the capabilities these First Nations need to provide quality drinking water to their communities (AFN, 2010b).

Change Model: An ideal map of specific interventions and activities for addressing a particular issue, as part of the theory of change (Connell and Kubisch, 1998). For the purposes of this study, it is a governance actor's ideal map of interventions and activities for addressing the overarching problem pertaining to drinking water quality in First Nations communities.

Contamination Risk: The susceptibility of a community's drinking water source for becoming tainted by the natural environment (e.g., animal waste, weather patterns), human intervention (e.g., faulty infrastructure development, industrial works such as mining) or waste. Risk for contamination can occur at any point throughout a drinking water system (Neegan Burnside, 201 la). Contamination risk is typically evaluated on a scale (see high risk, medium risk, and low risk).

Drinking Water Advisories (DWA): Several types of warnings for preparation of water before public consumption including: Boil-Water Advisory, Do Not Consume Advisory, Do Not Use Advisory (HC, 2012b).

First Nations Community: For the purposes of this study, First Nations communities consist of all First Nations reserve communities as recognized under the provisions of the Indian Act, 1985.

Governance Actors: Four government entities that contribute to the oversight of various high-level aspects of drinking water quality within First Nations communities. These actors include: Aboriginal Affairs and Northern Development Canada, Environment Canada, Health Canada, and First Nations governments (i.e., Chief and Council) (INAC, 2010a; HC, 201 la; Swain, Loutit, \& Hrudey, 2006)

High Risk: These are systems with major deficiencies, which-individually or combined-pose a high risk to the quality of water. These deficiencies may lead to potential health and safety or environmental concerns. They could also result in water quality advisories against drinking the water (such as, but not limited to, boil water advisories), repetitive non-compliance with guidelines, and inadequate water supplies. Once systems are classified under this category, regions and First Nations must take immediate corrective action to minimize or eliminate deficiencies (Neegan Burnside, 2011 ).

Infrastructure Gap: The difference between the current drinking water infrastructure in First Nations communities and the infrastructure these First Nations need to provide quality drinking water to their communities (CoO, 2008a). 
Low Risk: These are systems that operate with minor deficiencies. Low-risk systems usually meet the water quality parameters that are specified by the appropriate Guidelines water (in particular, the Guidelines for Canadian Drinking Water Quality) (Neegan Burnside, 2011 a).

Medium Risk: These are systems with deficiencies, which-individually or combinedpose a medium risk to the quality of water and to human health. These systems do not generally require immediate action, but the deficiencies should be corrected to avoid future problems (Neegan Burnside, 2011 a).

National Assessment of First Nations Water and Wastewater Systems: The National Assessment is a rigorous and comprehensive assessment of water and wastewater systems serving 571 participating First Nations that was conducted between 2009 and 2011. The assessment includes a report on each First Nation community as well as eight regional roll-up reports and one national roll-up report. AANDC developed the measures used and procured Neegan Burnside to conduct the assessment. The individual community reports are written and accredited to engineers who were contracted by Neegan Burnside.

Neegan Burnside Report: see National Assessment of First Nations Water and Wastewater Systems

Non-First Nations Community: For the purposes of this study, non-First Nations communities consist of all non-First Nations reserve communities.

Regulatory Gap: The difference between First Nations and non-First Nations communities with regard to drinking water regulations. Specifically, provinces legally enforce drinking water quality standards through passed legislation. By contrast, there are no such legally enforceable standards for First Nations communities. Therefore, there is said to be a gap with regard to First Nations and non-First Nations communities' drinking water regulations (AFN, 2008a).

Resource Gap: The difference between the level of financial and human resources currently available to First Nations communities for drinking water and level of financial and human resources these First Nations need to provide quality drinking water to their communities (AFN, 2012b).

Risk: (see Contamination Risk)

Theory of Action (Action Theory): How a set of program or intervention activities are devised by the program planners, decision-makers, and or implementers (Chen, 2005).

Theory of Change (Change Theory): A series of interventions and/or activities designed to address a particular issue (Connell and Kubisch, 1998). For the purposes of this thesis, it is the description of how to change the status quo of poor drinking water of First Nations communities to better water quality. 


\section{Annex 1: Departments/Ministries Responsible for Drinking}

Water Quality by Province/Territory

\begin{tabular}{ll}
\hline Province/Territory & Department/Ministry \\
\hline Alberta & Department of Environment \\
British Columbia & Ministry of Health Services \\
Manitoba & Department of Water Conservation \\
New Brunswick & Department of Health and Wellness \\
Newfoundland and Labrador & Department of Environment and Conservation \\
Northwest Territories & Stanton Territorial Health Authority \\
Nova Scotia & Department of Environment and Labour \\
Nunavut Territory & Department of Health and Social Sciences \\
Ontario & Ministry of the Environment \\
Prince Edward Island & Department of Environment, Energy, and Forestry \\
Quebec & Ministère du Développement durable, de \\
& l'Environnement et des Parcs \\
Saskatchewan & Department of the Environment \\
Yukon Territory & Department of Health and Social Sciences \\
\hline
\end{tabular}




\section{Annex 2: Frequency of Factors Pertaining to Specific First Nations}

\section{Drinking Water Quality Referenced by Governance Actor}

\begin{tabular}{|c|c|c|c|c|}
\hline \multirow{2}{*}{ Factor } & \multicolumn{4}{|c|}{ Governance Actor } \\
\hline & AANDC & $\mathrm{EC}$ & $\mathrm{HC}$ & $\mathrm{AFN} / \mathrm{CoO}$ \\
\hline \multicolumn{5}{|l|}{ Protection } \\
\hline Protect & 136 & 146 & 122 & 32 \\
\hline Pollution & 2 & 101 & 13 & 15 \\
\hline Source water & 106 & 20 & 104 & 17 \\
\hline Groundwater & 59 & 351 & 30 & 1 \\
\hline Surface water & 58 & 46 & 26 & 2 \\
\hline River & 13 & 475 & 7 & 15 \\
\hline Lake & 34 & 355 & 7 & 8 \\
\hline MTA & 8 & 0 & 0 & 0 \\
\hline \multicolumn{5}{|l|}{ Treatment } \\
\hline Treatment & 328 & 51 & 332 & 23 \\
\hline Disinfection & 115 & 5 & 169 & 0 \\
\hline Contamination & 38 & 108 & 123 & 10 \\
\hline Microbological & 78 & 5 & 60 & 0 \\
\hline Bacterial & 16 & 15 & 64 & 0 \\
\hline Protozoan & 6 & 0 & 16 & 0 \\
\hline E. coli & 1 & 1 & 43 & 0 \\
\hline Coliforms & 3 & 2 & 81 & 0 \\
\hline Chemical & 9 & 109 & 71 & 4 \\
\hline Operations & 271 & 39 & 95 & 27 \\
\hline Maintenance & 294 & 32 & 47 & 16 \\
\hline Emergency & 62 & 4 & 94 & 0 \\
\hline \multicolumn{5}{|l|}{ Research } \\
\hline Research & 10 & 88 & 25 & 4 \\
\hline Assessment & 235 & 49 & 92 & 11 \\
\hline Evaluation & 40 & 6 & 92 & 9 \\
\hline \multicolumn{5}{|l|}{ Monitoring } \\
\hline Monitor & 227 & 98 & 361 & 9 \\
\hline Sample & 238 & 13 & 481 & 4 \\
\hline Test & 228 & 27 & 168 & 8 \\
\hline \multicolumn{5}{|l|}{ Financial } \\
\hline Resources & 20 & 9 & 20 & 46 \\
\hline Funding & 158 & 16 & 18 & 26 \\
\hline Resource gap & 0 & 0 & 0 & 6 \\
\hline Capacity & 57 & 1 & 26 & 20 \\
\hline Capital & 66 & 26 & 3 & 17 \\
\hline Infrastructure & 125 & 15 & 17 & 32 \\
\hline Invest & 208 & 34 & 42 & 2 \\
\hline Design & 209 & 6 & 62 & 6 \\
\hline
\end{tabular}




\begin{tabular}{lcccc} 
Plan & 346 & 65 & 87 & 26 \\
Manuals & 30 & 4 & 268 & 2 \\
Protocols & 257 & 8 & 54 & 6 \\
Procedures & 61 & 6 & 405 & 2 \\
Guidelines & 93 & 68 & 178 & 6 \\
\hline WTOs & & & & \\
Training & 272 & 9 & 72 & 20 \\
Certification & 131 & 5 & 24 & 3 \\
Record keeping & 14 & 16 & 45 & 1 \\
Reporting & 32 & 32 & 113 & 7 \\
Retention & 5 & 2 & 14 & 5 \\
\hline Regulation & & & & \\
Regulations & 241 & 36 & 30 & 81 \\
Legislation & 143 & 17 & 34 & 60 \\
Compliance & 104 & 4 & 19 & 17 \\
Enforcement & 33 & 5 & 4 & 4 \\
\hline Engagement & & & & \\
Engage & 48 & 6 & 7 & 30 \\
Inform & 14 & 7 & 11 & 1 \\
Consult & 54 & 13 & 37 & 62 \\
Involve & 37 & 44 & 42 & 15 \\
Awareness & 31 & 18 & 42 & 3 \\
Understand & 5 & 80 & 24 & 4 \\
Accommodation & 0 & 2 & 0 & 12 \\
Educate & 12 & 7 & 21 & 8 \\
\hline Rights & & & & 37 \\
Treaty & 12 & 12 & 0 & 113 \\
Rights & 12 & 8 & 0 & 61 \\
Roles & 43 & 54 & 41 & 973 \\
Responsibilities & 115 & 31 & 66 & \\
\hline Total factor & 5,603 & 2,812 & 4,549 & \\
references & & & & 617 \\
\hline
\end{tabular}


Annex 3: Percentage of Total Factor References Pertaining to First Nations

Drinking Water Quality as Referenced by each Governance Actor

\begin{tabular}{|c|c|c|c|c|}
\hline \multirow{2}{*}{ Factor } & \multicolumn{4}{|c|}{ Governance Actor } \\
\hline & AANDC & $\mathrm{EC}$ & $\mathrm{HC}$ & AFN/CoO \\
\hline \multicolumn{5}{|l|}{ Protection } \\
\hline Protect & 2.4 & 5.2 & 2.7 & 3.3 \\
\hline Pollution & 0.0 & 3.6 & 0.3 & 1.5 \\
\hline Source water & 1.9 & 0.7 & 2.3 & 1.7 \\
\hline Groundwater & 1.1 & 12.5 & 0.7 & 0.1 \\
\hline Surface water & 1.0 & 1.6 & 0.6 & 0.2 \\
\hline River & 0.2 & 16.9 & 0.2 & 1.5 \\
\hline Lake & 0.6 & 12.6 & 0.2 & 0.8 \\
\hline MTA & 0.1 & 0.0 & 0.0 & 0.0 \\
\hline \multicolumn{5}{|l|}{ Treatment } \\
\hline Treatment & 5.9 & 1.8 & 7.3 & 2.4 \\
\hline Disinfection & 2.1 & 0.2 & 3.7 & 0.0 \\
\hline Contamination & 0.7 & 3.8 & 2.7 & 1.0 \\
\hline Microbological & 1.4 & 0.2 & 1.3 & 0.0 \\
\hline Bacterial & 0.3 & 0.5 & 1.4 & 0.0 \\
\hline Protozoan & 0.1 & 0.0 & 0.4 & 0.0 \\
\hline E. coli & 0.0 & 0.0 & 0.9 & 0.0 \\
\hline Coliforms & 0.1 & 0.1 & 1.8 & 0.0 \\
\hline Chemical & 0.2 & 3.9 & 1.6 & 0.4 \\
\hline Operations & 4.8 & 1.4 & 2.1 & 2.8 \\
\hline Maintenance & 5.2 & 1.1 & 1.0 & 1.6 \\
\hline Emergency & 1.1 & 0.1 & 2.1 & 0.0 \\
\hline \multicolumn{5}{|l|}{ Research } \\
\hline Research & 0.2 & 3.1 & 0.5 & 0.4 \\
\hline Assessment & 4.2 & 1.7 & 2.0 & 1.1 \\
\hline Evaluation & 0.7 & 0.2 & 2.0 & 0.9 \\
\hline \multicolumn{5}{|l|}{ Monitoring } \\
\hline Monitor & 4.1 & 3.5 & 7.9 & 0.9 \\
\hline Sample & 4.2 & 0.5 & 10.6 & 0.4 \\
\hline Test & 4.1 & 1.0 & 3.7 & 0.8 \\
\hline \multicolumn{5}{|l|}{ Financial } \\
\hline Resources & 0.4 & 0.3 & 0.4 & 4.7 \\
\hline Funding & 2.8 & 0.6 & 0.4 & 2.7 \\
\hline Resource gap & - & 0.0 & 0.0 & 0.6 \\
\hline Capacity & 1.0 & 0.0 & 0.6 & 2.1 \\
\hline Capital & 1.2 & 0.9 & 0.1 & 1.7 \\
\hline Infrastructure & 2.2 & 0.5 & 0.4 & 3.3 \\
\hline Invest & 3.7 & 1.2 & 0.9 & 0.2 \\
\hline Design & 3.7 & 0.2 & 1.4 & 0.6 \\
\hline
\end{tabular}

Documents 


\begin{tabular}{lcccc} 
Plan & 6.2 & 2.3 & 1.9 & 2.7 \\
Manuals & 0.5 & 0.1 & 5.9 & 0.2 \\
Protocols & 4.6 & 0.3 & 1.2 & 0.6 \\
Procedures & 1.1 & 0.2 & 8.9 & 0.2 \\
Guidelines & 1.7 & 2.4 & 3.9 & 0.6 \\
\hline WTOs & & & & \\
Training & 4.9 & 0.3 & 1.6 & 2.1 \\
Certification & 2.3 & 0.2 & 0.5 & 0.3 \\
Record keeping & 0.2 & 0.6 & 1.0 & 0.1 \\
Reporting & 0.6 & 1.1 & 2.5 & 0.7 \\
Retention & 0.1 & 0.1 & 0.3 & 0.5 \\
\hline Regulation & & & & \\
Regulations & 4.3 & 1.3 & 0.7 & 8.3 \\
Legislation & 2.6 & 0.6 & 0.7 & 6.2 \\
Compliance & 1.9 & 0.1 & 0.4 & 1.7 \\
Enforcement & 0.6 & 0.2 & 0.1 & 0.4 \\
\hline Engagement & & & & \\
Engage & 0.9 & 0.2 & 0.2 & 3.1 \\
Inform & 0.2 & 0.2 & 0.2 & 0.1 \\
Consult & 1.0 & 0.5 & 0.8 & 6.4 \\
Involve & 0.7 & 1.6 & 0.9 & 1.5 \\
Awareness & 0.6 & 0.6 & 0.9 & 0.3 \\
Understand & 0.1 & 2.8 & 0.5 & 0.4 \\
Accommodation & - & 0.1 & 0.0 & 1.2 \\
Educate & 0.2 & 0.2 & 0.5 & 0.8 \\
\hline Rights & & & & \\
Treaty & 0.2 & 0.4 & 0.0 & 3.8 \\
Rights & 0.2 & 0.3 & 0.0 & 11.6 \\
Roles & 0.8 & 1.9 & 0.9 & 1.7 \\
Responsibilities & 2.1 & 1.1 & 1.5 & 6.3 \\
\hline Total factor & 5,603 & 2,812 & 4,549 & 973 \\
references & & & & \\
\hline$\quad$ & & & \\
\hline
\end{tabular}




\section{Annex 4: Reference list for Documents Reviewed from}

\section{Aboriginal Affairs and Northern Development Canada}

AANDC. (2010). Transfer Payments. Retrieved from http://www.aadncaandc.gc.ca/eng/1100100010035.

AANDC. (2011). Backgrounder - Canada-Ontario First Nations Pilot to Improve Drinking Water Quality. Retrieved from http://www.aadncaandc.gc.ca/eng/1314383960721.

AANDC. (2011). Backgrounder - Government of Canada Investments in Water and Wastewater on First Nations in Manitoba. Retrieved from http://www.aadncaandc.gc.ca/eng/1313774991161.

AANDC. (2011). Backgrounder - National Engineering Assessment of First Nations Water and Wastewater Systems. Retrieved from http:/www.aadncaandc.gc.ca/aiarch/mr/nr/m-a2009/bk000000333-eng.asp.

AANDC. (2011). Backgrounder - Water and Wastewater Services in the Island Lake Region. Retrieved from http://www.aadnc-aandc.gc.ca/eng/1313772465863.

AANDC. (2011). Department's Response to the National Assessment of First Nations Water and Wastewater Systems. Retrieved from http:/www.aadncaandc.gc.ca/eng/1314383237592.

AANDC. (2011). Fact Sheet - Capacity and Training and Circuit Rider Training Program. Retrieved from http://www.aadnc-aandc.gc.ca/eng/1313688917297.

AANDC. (2011). Fact Sheet - Infrastructure Investments and Investment Planning. Retrieved from http://www.aadnc-aandc.gc.ca/eng/1313688016644.

AANDC. (2011). Fact Sheet - Risk Assessment of Water and Wastewater Systems in First Nations Communities. Retrieved from http://www.aadncaandc.gc.ca/eng/1313687144247

AANDC. (2011). Fact Sheet - Roles and Responsibilities Water Assessment and Monitoring. Retrieved from http://www.aadnc-aandc.gc.ca/eng/1313690232354.

AANDC. (2011). Fact Sheet - Understanding the Results of the National Assessment. Retrieved from http://www.aadnc-aandc.gc.ca/eng/1313683655131.

AANDC. (2011). Fact Sheet - Water and Wastewater Protocols. Retrieved from http://www.aadnc-aandc.gc.ca/eng/1313685676730.

AANDC. (2011). Fact Sheet - Water Quality. Retrieved from http://www.aadncaandc.gc.ca/eng/1100100035034. 
AANDC. (2011). First Nations Water and Wastewater Action Plan. Retrieved from http://www.aadnc-aandc.gc.ca/eng/1313426171775

AANDC. (2011). Frequently Asked Questions - National Assessment of Water and Wastewater Systems in First Nation Communities: Release of Final Reports. Retrieved from http://www.aadnc-aandc.gc.ca/eng/1313691954022.

AANDC. (2011). Frequently Asked Questions: Water School Kit for First Nations Children. Retrieved from http://www.aadnc-aandc.gc.ca/aiarch/mr/nr/j-a2007/22854-faq-eng.asp.

AANDC. (2011). Funding Agreement National Model for Use with Aboriginal Recipients: Version for First Nations and Tribal Councils for 2011-2012.

AANDC. (2011). Infrastructure Investments in First Nations Water and Wastewater Systems. Retrieved from http://www.aadnc-aandc.gc.ca/eng/1313422821063.

AANDC. (2011). Minister Duncan Releases National Assessment of First Nations Water and Wastewater Systems Results. Retrieved from http://www.aadncaandc.gc.ca/eng/1314731838671.

AANDC. (2011). Minister Strahl announces contract to assess water and wastewater systems for First Nation communities. Retrieved from http://www.aadncaandc.gc.ca/aiarch/mr/nr/m-a2009/nr000000334-eng.asp

AANDC. (2011). Pilot Project Marks Important Partnership to Improve on Reserve Water Quality in Ontario. Retrieved from http://www.aadncaandc.gc.ca/eng/1314382852053.

AANDC. (2012). Backgrounder - Safe Drinking Water for First Nations Act. Retrieved from http://www.aadnc-aandc.gc.ca/eng/1330529331921

AANDC. (2012). Frequently Asked Questions - Safe Drinking Water for First Nations Act. Retrieved from http://www.aadnc-aandc.gc.ca/eng/1330528850798

AANDC. (2012). Harper Government Introduces Updated Legislation to Protect Drinking Water in First Nation Communities. Retrieved from http://www.aadncaandc.gc.ca/eng/1330541162174

AANDC. (2012). Safe Drinking Water for First Nations Act. Retrieved from http://www.aadnc-aandc.gc.ca/eng/1330528512623

INAC. (2003). National assessment of water and wastewater systems in First Nations communities - Summary report.

INAC. (2006). Backgrounder Priority List of First Nation Communities with High Risk Water Systems and Drinking Water Advisories. Retrieved from http://www.aadncaandc.gc.ca/aiarch/mr/nr/j-a2006/02757bk-eng.asp 
INAC. (2006). Effective Action on First Nations Water. Retrieved from http://www.aadnc-aandc.gc.ca/aiarch/mr/nr/j-a2006/02757fbk-eng.asp.

INAC. (2006). Frequently Asked Questions: Improving Water Quality On-reserve. Retrieved from http://www.aadnc-aandc.gc.ca/aiarch/mr/nr/j-a2006/02757ebkeng.asp.

INAC. (2006). Fact Sheet: Improving Water Quality On-Reserve: Assessing Risk on Drinking Water Systems. Retrieved from http://www.aadncaandc.gc.ca/aiarch/mr/nr/j-a2006/02757abk-eng.asp

INAC. (2006). Fact Sheet: Improving Water Quality On-reserve: New Protocol for Safe Drinking Water in First Nations Communities. Retrieved from http://www.aadncaandc.gc.ca/aiarch/mr/nr/j-a2006/02757dbk-eng.asp

INAC. (2006). Fact Sheet: Improving Water Quality On-reserve: Operators training and certification. Retrieved from http://www.aadnc-aandc.gc.ca/aiarch $/ \mathrm{mr} / \mathrm{nr} / \mathrm{j}$ a2006/02757cbk-eng.asp

INAC. (2006). Fact Sheet: Improving Water Quality On-reserve: Roles and Responsibilities. Retrieved from http://www.aadnc-aandc.gc.ca/aiarch/mr/nr/ja2006/02757bbk-eng.asp.

INAC. (2006). Government Announces Immediate Action on First Nations Drinking Water. Retrieved from http://www.aadnc-aandc.gc.ca/aiarch/mr/nr/j-a2006/202757-eng.asp.

NAC. (2006). Plan of action for drinking water in First Nations communities: A progress report.

INAC. (2006). Protocol for Safe Drinking Water in First Nations Communities. Retrieved from http://www.aadnc-aandc.gc.ca/DAM/DAM-INTERHQ/STAGING/texte-text/sdw_1100100015850_eng.pdf.

INAC. (2007). Plan of action for drinking water in First Nations communities: A progress report.

INAC. (2008). Backgrounder - First Nations Water and Wastewater Action Plan. Retrieved from http://www.aadnc-aandc.gc.ca/aiarch/mr/nr/j-a2008/2-3019-bkeng.asp.

INAC. (2008). Government Of Canada Announces Next Important Steps To Improve Drinking Water In First Nations Communities. Retrieved from http://www.aadncaandc.gc.ca/aiarch/mr/nr/j-a2008/2-3019-eng.asp

INAC. (2008). Plan of action for drinking water in First Nations communities: $A$ progress report. 
INAC. (2009). Discussion Paper: Engagement Sessions on the Development of a Proposed Legislative Framework for Drinking Water and Wastewater in First Nation Communities. Retrieved from http://www.aadnc-aandc.gc.ca/DAM/DAMINTER-HQ/STAGING/texte-text/dsp_1100100034904_eng.pdf.

INAC. (2009). Engagement Sessions on the Development of a Proposed Legislative Framework for Drinking Water and Wastewater in First Nation Communities. Retrieved from http://www.aadnc-aandc.gc.ca/eng/1100100034897.

INAC. (2009). First Nations water and wastewater action plan: Progress report January 2008-March 2009.

INAC. (2009). Government of Canada Takes More Action to Clean Up Drinking Water in First Nation Communities. Retrieved from http://www.aadncaandc.gc.ca/aiarch/mr/nr/j-a2009/nr000000168-eng.asp.

INAC. (2010). Chronology - Water. Retrieved from http://www.aadncaandc.gc.ca/eng/1308332537951.

INAC. (2010). INAC's Support for First Nations Water and Wastewater Infrastructure April 1, 2006 - March 31, 2010. Retrieved from http://www.aadncaandc.gc.ca/eng/1313696742768.

INAC. (2010). Protocol for Centralised Drinking Water Systems in First Nations Communities: Protocol for Centralised Drinking Water Systems in First Nations Communities. Retrieved from http://www.aadnc-aandc.gc.ca/eng/1100100034998.

INAC. (2010). Protocol for Decentralised Drinking Water Systems in First Nations Communities: Protocol for Centralised Drinking Water Systems in First Nations Communities. Retrieved from http://www.aadnc-aandc.gc.ca/eng/1 100100034998.

INAC. (2010). Water and wastewater infrastructure: Investment report April 2006March 2010.

INAC \& HC. (2007). Summative Evaluation of the First Nations Water Management Strategy. Retrieved from http://www.aincinac.gc.ca/ai/arp/aev/pubs/ev/efnw08/efnw08-eng.asp 


\section{Annex 5: Reference list for Documents Reviewed from Environment Canada}

Bruce, J., \& B. Mitchell. (1995). Broadening Perspectives on Water Issues. Canadian Global Change Incidental Report Series No., IR95-1. Retrieved from http://www.ec.gc.ca/eau-water/default.asp?lang=En\&n=5593BDE01 \&wsdoc=60A25212-85D2-45F6-92F8-C2BFFA3E3488\&category=DA4E869156B5-4DE9-A101-1F35C62C409A

EC. (1992). Clean Water - Life Depends on It! Retrieved from http://www.ec.gc.ca/eauwater/default.asp?lang $=$ En\&n $=06 \mathrm{~F} 1403 \mathrm{C}-1$

EC. (1999). Groundwater - Nature's Hidden Treasure. Retrieved from http://www.ec.gc.ca/eau-water/default.asp?lang=En\&n=3F93145A-1

EC. (2001). Water, Art, and the Canadian Identity: At the Water's Edge. Retrieved from http://www.ec.gc.ca/eau-water/default.asp?lang=en\&n=62D086E9-1

EC. (2003). Water and Canada: Preserving a Legacy for People and the Environment. Retrieved from http://www.ec.gc.ca/Publications/AFF1D423-17C8-4E20-BA0F91773EF7356F\%5CWater-and-Canada-Preserving-a-Legacy-for-People-and-theEnvironment.pdf

EC. (2006). Water - How good is it? Retrieved from http://www.ec.gc.ca/eauwater/default.asp?lang=En\&n=1A0A0DC6-1

EC. (2006). Water - How We Manage It. Retrieved from http://www.ec.gc.ca/eauwater/default.asp?lang=En\&n=3DC41CC0-1

EC. (2006). Water -How We Share It. Retrieved from http://www.ec.gc.ca/eauwater/default.asp?lang=En\&n=746FC7D7-1

EC. (2006). Water -In Canada. Retrieved from http://www.ec.gc.ca/eauwater/default.asp?lang=En\&n=5EA ID86E-1

EC. (2009). Integrated Water Monitoring. Retrieved from http://www.ec.gc.ca/scitech/default.asp?lang=En\&n=B0EFF0EC-1

EC. (2010). Fresh Water Quality Monitoring. Retrieved from http://www.ec.gc.ca/eaudouce-freshwater/Default.asp?lang=En

EC. (2011). Drinking Water. Retrieved from http://www.ec.gc.ca/eauwater/default.asp?lang=En\&n=AAD0 ICB4-1.

EC. (2011). Federal Policy and Legislation. Retrieved from http://www.ec.gc.ca/eauwater/default.asp?lang=En\&n=E05A7F81-1.

EC. (2011). Frequently Asked Questions. Retrieved from http://www.ec.gc.ca/eauwater/default.asp?lang=En\&n=1C100657-1. 
EC. (2011). Fresh Water Quality Monitoring. Retrieved from http://www.ec.gc.ca/eaudouce-freshwater/Default.asp?lang=En.

EC. (2011). Groundwater. Retrieved from http://www.ec.gc.ca/eauwater/default.asp?lang=En\&n $=300688 \mathrm{DC}-1$.

EC. (2011). GEMS Water. Retrieved from http://www.ec.gc.ca/qualitedeleauwaterquality/default.asp?lang=En\&n=4E469266-1.

EC. (2011). Introduction to Water Quality. Retrieved from http://www.ec.gc.ca/eauwater/default.asp?lang=En\&n=2C3144F5-1.

EC. (2011). Lakes. Retrieved from http://www.ec.gc.ca/eauwater/default.asp?lang $=$ En\& $=448 \mathrm{~F} 99 \mathrm{~F} 0-1$.

EC. (2011). Quickfacts. Retrieved from http://www.ec.gc.ca/eauwater/default.asp?lang=En\&n=11A8CA33-1.

EC. (2011). Rivers. Retrieved from http://www.ec.gc.ca/eauwater/default.asp?lang=En\&n=45BBB7B8- 1 .

EC. (2011). Water and Canada's Aboriginal Peoples. Retrieved from http://www.ec.gc.ca/eau-water/default.asp?lang=En\&n=BA5125BF-1.

EC. (2011). Water and the Canadian Identity. Retrieved from http://www.ec.gc.ca/eauwater/default.asp?lang=En\&n=5593BDE0-1.

EC. (2011). Water Governance and Legislation. Retrieved from http://www.ec.gc.ca/eauwater/default.asp?lang $=$ En\&n=87922E $3 \mathrm{C}-1$.

EC. (2011). Water Quality Monitoring. Retrieved from http://www.ec.gc.ca/qualitedeleauwaterquality/default.asp?lang=En\&n=0DF8E8D2-1.

EC. (2011). Water Quality Objectives and Guidelines. Retrieved from http://www.ec.gc.ca/eau-water/default.asp?lang=En\&n=F77856A7-1

EC. (2012). About Environment Canada. http://www.ec.gc.ca/default.asp?lang=En\&n=BD3CE17D-1.

EC. (2012). Comprehensive Approach to Clean Water. Retrieved from http://www.ec.gc.ca/eau-water/default.asp?lang=En\&n=B1 128A3D-1

EC. (2012). Groundwater Contamination. Retrieved from http://www.ec.gc.ca/eauwater/default.asp?lang $=$ En\&n=6A7FB7B2-1

EC. (2012). Shared Responsibility. Retrieved from http://www.ec.gc.ca/eauwater/default.asp?lang $=$ En\&n=035F6173-1. 
EC. (2012). Water Management. Retrieved from http://www.ec.gc.ca/eauwater/default.asp?lang=En\&n=DF9EE875-1

EC. (2012). Water Quality. Retrieved from http://www.ec.gc.ca/eauwater/default.asp?lang $=$ En\&n=F2F43FC7-1

McDiarmid, M. (2012). Budget bill makes complex changes to fisheries protection: Greens leader Elizabeth May fears legislation will eliminate habitat protection. Retrieved from http://www.cbc.ca/news/politics/story/2012/05/03/pol-budget-billfisheries.html 


\section{Annex 6: Reference list for Documents Reviewed from Health Canada}

HC. (2007). Procedure for Addressing Drinking Water Advisories in First Nations Communities South of $60^{\circ}$. Retrieved from http://www.hc-sc.gc.ca/fniahspnia/alt_formats/fnihb-dgspni/pdf/pubs/water-eau/2007_water-qualit-eaueng.pdf.

HC. (2007). Procedure Manual for Safe Drinking Water in First Nations Communities South of $60^{\circ}$. Retrieved from http://www.hc-sc.gc.ca/fniahspnia/pubs/promotion/_environ/manual-manuel/index-eng.php.

HC. (2009). Drinking Water Advisories in First Nations Communities in Canada - A National Overview 1995-2007. Retrieved from http://www.hc-sc.gc.ca/fniahspnia/pubs/promotion/_environ/2009_water-qualit-eau-canada/index-eng.php.

HC. (2009). Protecting the Water in your Pipes. Retrieved from http://www.hcsc.gc.ca/fniah-spnia/alt_formats/fnihb-dgspni/pdf/pubs/promotion/pipe-conduiteeng.pdf.

HC. (2010). A Guide for Environmental Health Officers and Community-Based Drinking Water Monitors. Retrieved from http://www.hc-sc.gc.ca/fniahspnia/alt_formats/pdf/pubs/promotion/environ/individ-wells-puit/guide-eng.pdf.

HC. (2010). Boil your water. Retrieved from http://www.hc-sc.gc.ca/fniahspnia/pubs/promotion/_environ/watk-toaql/boil-bouillir-eng.php

HC. (2010). Do not drink. Retrieved from http://www.hc-sc.gc.ca/fniahspnia/pubs/promotion/_environ/watk-toaql/donot-nepas-eng.php

HC. (2010). Ensuring Safe Drinking Water in First Nations Communities in Canada. Retrieved from http:/www.hc-sc.gc.ca/fniah-spnia/promotion/publicpublique/sfw-sep-eng.php.

HC. (2010). Inspecting Your Well: A Step-by-Step Checklist. Retrieved from http://www.hc-sc.gc.ca/fniah-spnia/pubs/promotion/_environ/individ-wellspuits/insp-well-puit-eng.php.

HC. (2010). Safe to consume. Retrieved from http://www.hc-sc.gc.ca/fniahspnia/pubs/promotion/_environ/watk-toaql/safe-bonne-eng.php.

HC. (2010). Toolkit for Individual Wells. Retrieved from http://www.hc-sc.gc.ca/fniahspnia/pubs/promotion/_environ/individ-wells-puits/index-eng.php

HC. (2010). Testing Your Well Water: A Step-by-Step Checklist. Retrieved from http://www.hc-sc.gc.ca/fniah-spnia/pubs/promotion/_environ/individ-wellspuits/test-analyse-well-puit-eng.php. 
HC. (2011). Drinking water and wastewater. Retrieved from http://www.hcsc.gc.ca/fniah-spnia/promotion/public-publique/water-eau-eng.php.

HC. (2011). First Nations, Inuit and Aboriginal Health. Retrieved from http://www.hcsc.gc.ca/fniah-spnia/pubs/promotion/index-eng.php.

HC. (2011). Procedural Guidelines for Waterborne Disease Events in First Nations Communities South of $60^{\circ}$. Retrieved from http://www.hc-sc.gc.ca/fniahspnia/pubs/promotion/_environ/south-sud-60/index-eng.php.

HC. (2012). About Health Canada. Retrieved from http://www.hc-sc.gc.ca/ahcasc/index-eng.php.

INAC \& HC. (2007). Summative Evaluation of the First Nations Water Management Strategy. Retrieved from http://www.aincinac.gc.ca/ai/arp/aev/pubs/ev/efnw08/efnw08-eng.asp 


\section{Annex 7: Reference list for Documents Reviewed from the Assembly of First Nations and Chiefs of Ontario}

AFN. (2008). Letter from National Chief to Minister Strahl Regarding the Continued Unsatisfactory State of Access to Safe Drinking Water Experienced by Many First Nations People. Retrieved from http://www.afn.ca/uploads/files/water/08-0322.pdf.

AFN. (2008). Letter from Richard Jock, AFN to Karl Carisse, INAC Regarding Serious Objections to the Unilateral Approach to Consultation and the Pursuit on the Single Option Regarding the Proposed Legislation for Application to First Nations communities. Retrieved from http://www.afn.ca/uploads/files/water/0808 -2.pdf.

AFN. (2009). Aboriginal Water Rights Primer. Retrieved from http://www.afn.ca/uploads/files/water-primer.pdf

AFN. (2010). AFN News Bulletin - AFN National Chief Calls for Real Action on Safe Drinking Water for First Nations: Need Action to Address the "Capacity Gap as well as the Regulatory Gap". Retrieved from http://www.afn.ca/uploads/files/water/10-05-27.pdf.

AFN. (2010). Briefing note: Bill S-11, Safe drinking water for First Nations Act. Retrieved from http://www.afn.ca/uploads/files/bill-s-11.pdf.

AFN. (2010e). Canada-Wide Strategy for the Management of Municipal Wastewater Effluent and Environment Canada's Proposed Regulatory Framework for Wastewater: Impacts for First Nations Communities. Retrieved from http://www.afn.ca/uploads/files/regional-impact-analysis.pdf

AFN. (2010). Letter from National Chief Atleo to Minister Duncan Conveying that Bills $S$-11 is Not Acceptable in its Current Form. Retrieved from http://www.afn.ca/uploads/files/water/10-11-30.pdf.

AFN. (2010). Letter from National Chief Atleo to the Standing Committee on Aboriginal Peoples Regarding An Act Respecting the Safety of Drinking Water on First Nation Lands (Bill S-11). Retrieved from http://www.afn.ca/uploads/files/water/10-09-30.pdf.

AFN. (2010). Resolution: Bill S-11, Safe drinking water for First Nations Act. Retrieved from http://www.afn.ca/uploads/files/water/10-12-14.pdf.

AFN. (2010). Update on First Nations Drinking Water. Retrieved from http://www.afn.ca/uploads/files/water/10-06-00.pdf

AFN. (2011). Water and Wastewater. Retrieved from http://www.afn.ca/index.php/en/policy-areas/water. 
AFN. (2011). Assembly of First Nations National Chief Welcomes Federal Commitment to Drinking Water, Urges Full engagement of First Nations. Retrieved from http://www.afn.ca/index.php/en/news-media/latest-news/assembly-of-firstnations-national-chief-welcomes-federal-commitment- $t$

AFN. (2011). Assembly of First Nations National Chief Commends Parliaments Support for Clean Drinking Water for First Nations, Urges Full Engagement of First Nations. Retrieved from http://www.afn.ca/index.php/en/news-media/latestnews/assembly-of-first-nations-national-chief-commends-parliaments-support-

AFN. (2011). Communique from National Chief Shawn Atleo - August, 2011. Retrieved from http://www.afn.ca/index.php/en/news-media/latest-news/communique-fromnational-chief-shawn-atleo-august-2011

AFN. (2011). AFN Responds to the National Assessment of First Nation Water and Wastewater System Report. Retrieved from http://www.afn.ca/index.php/en/news-media/latest-news/afn-responds-to-thenational-assessment-of-first-nation-water-and-wast

AFN. (2011). AFN Supports Pikangikum First Nations Call for New Water Supply System: State of Emergency. Retrieved from http://www.afn.ca/index.php/en/news-media/latest-news/afn-supportspikangikum-first-nations-call-for-new-water-supply-system

CoO. (2004). Update on Watershed-based Source Protection. Retrieved from http://chiefs-of-ontario.org/PageContent/Default.aspx?SectionHeadlineID=119.

CoO. (2004). Watershed-based Source Protection. Retrieved from http://chiefs-ofontario.org/PageContent/Default.aspx?SectionHeadlinelD $=118$

CoO. (2005). Drinking Water in First Nations Communities. Retrieved from http://chiefsof-ontario.org/PageContent/Default.aspx?SectionHeadlineID=98.

CoO. (2005). Ontario Source Water Protection Act and Regime: Effect of Aboriginal and Treaty Rights and Interests. Retrieved from http://chiefs-ofontario.org/PageContent/Default.aspx?SectionHeadlinelD $=120$

CoO. (2005). The Soft Path for Water in a Nutshell. Retrieved from http://chiefs-ofontario.org/Assets/Water\%20Soft\%20Paths\%20in\%20a\%20Nutshell1.pdf

CoO. (2008). COO Bulletin Regarding Indian and Northern Affairs Canada (INAC) Proposed Legislation on Water and Wastewater. Retrieved from http://www.afn.ca/uploads/files/water/08-08-18.pdf.

CoO. (2008). COO Water Declaration of the First Nations in Ontario. Retrieved from http://www.afn.ca/uploads/files/water/08-10-00.pdf 
CoO. (2008). Letter from COO to Minister Strahl Expressing Concerns of First Nations in Ontario Regarding Approach with Respect to the Development of a Legislative Framework for Drinking Water and Wastewater in First Nations Communities. Retrieved from http://www.afn.ca/uploads/files/water/08-08-18.pdf.

CoO. (2009). COO Bulletin Regarding Indian and Northern Affairs Canada (INAC) Engagement Sessions on the Development of a Proposed Legislative Framework on Drinking Water and Wastewater in First Nation Communities. Retrieved from http://www.afn.ca/uploads/files/water/09-02 2.pdf

CoO. (2009). COO Bulletin Regarding Indian and Northern Affairs Canada (INAC) Proposed Legislative Framework on Drinking Water and Wastewater in First Nation Communities. Retrieved from http://www.afn.ca/uploads/files/water/0901-21.pdf.

CoO. (2009). Letter from COO to Minister Strahl Regarding INAC's Proposed Legislative Framework for Drinking Water and Wastewater in First Nations Communities. Retrieved from http://www.afn.ca/uploads/files/water/09-11-05.pdf.

CoO. (2010). COO Resolution Bill S-11 An Act Respecting the Safety of Drinking Water on First Nations. Retrieved from http:/www.afn.ca/uploads/files/water/10-1123.pdf.

CoO. (2010). Letter from COO to Senator St. Germain Expressing Concerns Regarding Bill S-11, An Act Respecting the Safety of Drinking Water on First Nations Lands. Retrieved from http://www.afn.ca/uploads/files/water/10-09-29.pdf.

CoO. (2011). Chiefs in Ontario Strategies to Implement the Water Declaration. Retrieved from http://chiefs-of-ontario.org/Assets/COO\%20declaration-strategies.pdf.

CoO. (2011). COO Long Form Declaration. http://chiefs-ofontario.org/Assets/COO\%20water\%20declaration\%20revised\%20march\%202010 .pdf. 
Annex 8: General Profiles of Interview Respondents

\begin{tabular}{lccc}
\hline Organization & $\begin{array}{l}\text { \# of } \\
\text { interviewees }\end{array}$ & Interviewee & $\begin{array}{l}\text { Interview } \\
\text { Length }\end{array}$ \\
\hline $\begin{array}{l}\text { Governance Actors } \\
\text { AANDC }\end{array}$ & 1 & $\begin{array}{c}\text { Senior Managing Public } \\
\text { Servant from Ottawa }\end{array}$ & 1 hour \\
AFN/CoO & 1 & Senior \\
EC & 1 & $\begin{array}{c}\text { Senior Managing Public } \\
\text { Servant from Ottawa }\end{array}$ & 1.5 hours \\
HC & 2 & $\begin{array}{c}\text { Senior Managing Public } \\
\text { Servants from Ottawa }\end{array}$ & 2 hours \\
Communities & 1 & $\begin{array}{c}\text { Senior Manager from the } \\
\text { OCW A in Longlac }\end{array}$ & 1 hour \\
OCWA - Longlac & 1 & Senior Managing Public \\
Ginoogaming & 1 & Servant from Ginoogaming \\
Greater Sudbury - & Senior WTO in Greater \\
Dowling & Sudbury & .5 hours \\
\hline
\end{tabular}




\section{Annex 9: Interview Questions Used for the Purposes of this Study}

1. What has been the primary issue with water quality in Canada?

2. How has the issue been different for individuals living in First Nations communities?

a. What specific factors have contributed to the issue?

b. Of those contributing factors you have mentioned which are the most important?

3. Has this issue persisted? If so, why?

4. What steps need to be taken to address this issue?

a. What specific activities must be completed?

b. How would you rank the activities mentioned in order of importance?

5. What specific goals will be achieved in completing the activities?

a. Why will these goals be achieved?

6. What assets are needed to fulfil the steps you suggested?

7. Are there any assumptions underlying the proposed steps for addressing the issue?

8. What is the role of your organization in the management of drinking water on First Nations reserves?

9. Are there any other ideas we have neglected to talk about? Are there other insights that you wish to provide that could better inform this research? 
Annex 10: Huey T. Chen's Action Theory Model $^{44}$

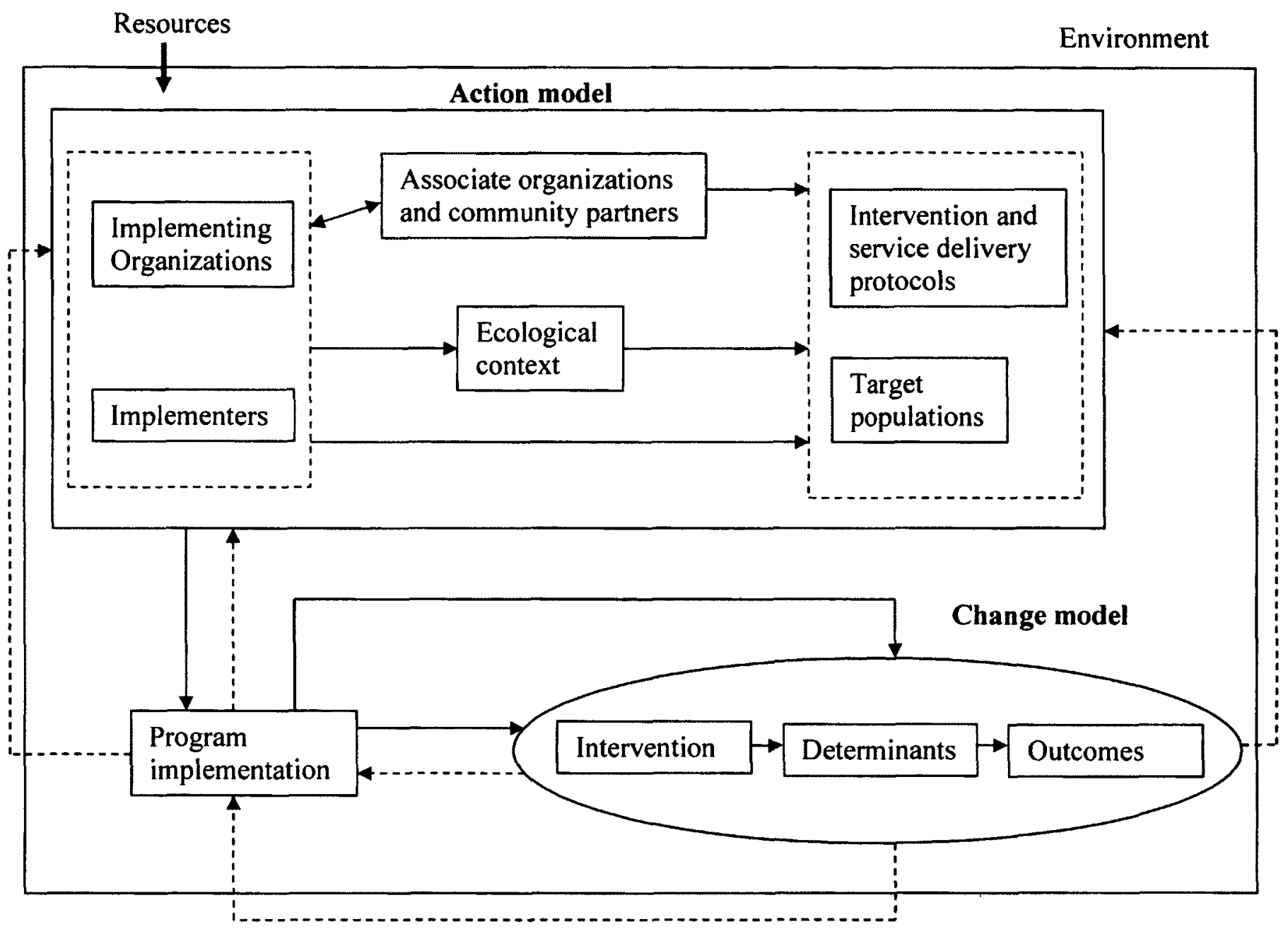

${ }^{44}$ Verbatim from Chen, H.T. (2005). Practical Program Evaluation: Assessing and Improving Planning, Implementation, and Effectiveness. Thousand Oaks, CA: Sage Publications Inc. 
Annex 11: Water Quality Parameters for Municipal Type Agreement Source

\begin{tabular}{|c|c|c|c|c|}
\hline Parameter & $\begin{array}{l}\text { Unit of } \\
\text { Measure }\end{array}$ & $\begin{array}{l}\text { Ginoogaming } \\
\text { First Nation }{ }^{43}\end{array}$ & $\begin{array}{l}\text { Longlac, in } \\
\text { Greenstone }^{46}\end{array}$ & $\begin{array}{l}\text { Maximum } \\
\text { Allowable } \\
\text { Concentrations }{ }^{47}\end{array}$ \\
\hline Alachlor & $\mathrm{mg} / \mathrm{L}$ & 0.0005 & 0.0001 & \\
\hline Aldicarb & $\mathrm{mg} / \mathrm{L}$ & 0.005 & 0.001 & 0.009 \\
\hline Aldrin + Deildrin & $\mathrm{mg} / \mathrm{L}$ & 0.00001 & 0.00004 & 0.0007 \\
\hline Alkalinity & $\mathrm{mg} / \mathrm{L}$ & 36 & - & $30-500$ \\
\hline Aluminum & $\mathrm{mg} / \mathrm{L}$ & 0.093 & - & $0.1 / 0.2$ \\
\hline Antimony & $\mathrm{mg} / \mathrm{L}$ & 0.0005 & 0.0006 & 0.006 \\
\hline Arsenic & $\mathrm{mg} / \mathrm{L}$ & 0.001 & 0.001 & 0.01 \\
\hline $\begin{array}{l}\text { Atrazine }+\mathrm{N}- \\
\text { dealkylated metabolites }\end{array}$ & $\mathrm{mg} / \mathrm{L}$ & 0.001 & 0.002 & 0.005 \\
\hline Azinphos-methyl & $\mathrm{mg} / \mathrm{L}$ & 0.002 & 0.0001 & 0.02 \\
\hline Barium & $\mathrm{mg} / \mathrm{L}$ & 0.008 & 0.01 & 1 \\
\hline Bendiocarb & $\mathrm{mg} / \mathrm{L}$ & 0.002 & 0.0002 & 0.04 \\
\hline Benzene & $\mathrm{mg} / \mathrm{L}$ & 0.0001 & 0.0005 & 0.005 \\
\hline Benzo[a]pyrene & $\mathrm{mg} / \mathrm{L}$ & 0.000009 & 0.00001 & 0.00001 \\
\hline Boron & $\mathrm{mg} / \mathrm{L}$ & 0.01 & 0.05 & 5 \\
\hline Bromodichloromethane & $\mathrm{mg} / \mathrm{L}$ & 0.0021 & 0.00001 & 0.01 \\
\hline Bromoform & $\mathrm{mg} / \mathrm{L}$ & 0.0001 & 0.0002 & 0.005 \\
\hline Bromoxynil & $\mathrm{mg} / \mathrm{L}$ & 0.0005 & 0.0002 & 0.005 \\
\hline Cadmium & $\mathrm{mg} / \mathrm{L}$ & 0.0001 & 0.0001 & 0.005 \\
\hline Carbon Tetrachloride & $\mathrm{mg} / \mathrm{L}$ & 0.0001 & 0.0005 & 0.005 \\
\hline Calcium & $\mathrm{mg} / \mathrm{L}$ & 32 & - & Archived $^{48}$ \\
\hline Carbaryl & $\mathrm{mg} / \mathrm{L}$ & 0.005 & 0.0002 & 0.09 \\
\hline Carbofuran & $\mathrm{mg} / \mathrm{L}$ & 0.005 & 0.0002 & 0.09 \\
\hline Carbon Tetrachloride & $\mathrm{mg} / \mathrm{L}$ & 0.0001 & 0.0005 & 0.005 \\
\hline Chlordane & $\mathrm{mg} / \mathrm{L}$ & 0.00001 & 0.0003 & Archived \\
\hline Chloride & $\mathrm{mg} / \mathrm{L}$ & 5 & - & 250 \\
\hline Chloroform & $\mathrm{mg} / \mathrm{L}$ & 0.0354 & - & 0.1 \\
\hline Chloropyrifos & $\mathrm{mg} / \mathrm{L}$ & 0.001 & 0.001 & 0.09 \\
\hline Chromium & $\mathrm{mg} / \mathrm{L}$ & 0.005 & 0.001 & 0.05 \\
\hline Coliform & CFU $/ 100 \mathrm{~mL}$ & 0 & 0 & 0 \\
\hline Color, True & T.C.U. & 2 & - & 15 \\
\hline Conductivity & umhos $/ \mathrm{cm}$ & 234 & - & - \\
\hline Copper & $\mathrm{mg} / \mathrm{L}$ & 0.039 & - & 1.0 \\
\hline Cyanazine & $\mathrm{mg} / \mathrm{L}$ & 0.001 & 0.001 & 0.01 \\
\hline Cyanide & $\mathrm{mg} / \mathrm{L}$ & 0.005 & - & 0.2 \\
\hline Diazinon & $\mathrm{mg} / \mathrm{L}$ & 0.001 & 0.001 & 0.02 \\
\hline
\end{tabular}

${ }^{45}$ Obtained from the Health Canada via the Neegan Burnside report (Salma, 2010)

${ }^{46}$ Obtained from Greenstone's website (OCWA, 2012a). These results are required to be public as per

O.Reg. 170/03 under the Safe Drinking Water Act, 2002.

${ }^{47}$ Obtained from the Guidelines of Canadian Drinking Water Quality: Summary Table (HC, 2010).

${ }^{48}$ Archived measures are no longer part of the GCDWQ. 


\begin{tabular}{|c|c|c|c|c|}
\hline Dicamba & $\mathrm{mg} / \mathrm{L}$ & 0.001 & 0.002 & 0.12 \\
\hline Dibromochoromethane & $\mathrm{mg} / \mathrm{L}$ & 0.0001 & - & - \\
\hline Dichorobenzene $(1,2)$ & $\mathrm{mg} / \mathrm{L}$ & 0.0002 & 0.0005 & 0.2 \\
\hline Dichorobenzene $(1,4)$ & $\mathrm{mg} / \mathrm{L}$ & 0.0002 & 0.0005 & 0.005 \\
\hline $\begin{array}{l}\text { Dichlorodiphenyltri- } \\
\text { chloroethane (DDT) + } \\
\text { metabolites }\end{array}$ & $\mathrm{mg} / \mathrm{L}$ & 0.00002 & 0.004 & Archived \\
\hline Dichloroethane $(1,2)$ & $\mathrm{mg} / \mathrm{L}$ & 0.0001 & 0.0005 & 0.005 \\
\hline Dichloroethylene $(1,1)$ & $\mathrm{mg} / \mathrm{L}$ & 0.0001 & 0.0005 & 0.014 \\
\hline Dichloromethane & $\mathrm{mg} / \mathrm{L}$ & 0.0005 & 0.0005 & 0.05 \\
\hline Dichlorophenol & $\mathrm{mg} / \mathrm{L}$ & 0.0005 & 0.0005 & 0.9 \\
\hline $\begin{array}{l}\text { Dichlorophenoxy }(2,4) \\
\text { acetic acid }\end{array}$ & $\mathrm{mg} / \mathrm{L}$ & 0.001 & 0.0002 & 0.1 \\
\hline Diclofop-methyl & $\mathrm{mg} / \mathrm{L}$ & 0.0009 & 0.0002 & 0.009 \\
\hline Dimethoate & $\mathrm{mg} / \mathrm{L}$ & 0.003 & 0.0001 & 0.02 \\
\hline Dinoseb & $\mathrm{mg} / \mathrm{L}$ & 0.001 & 0.0001 & 0.01 \\
\hline Diquat & $\mathrm{mg} / \mathrm{L}$ & 0.007 & 0.001 & 0.07 \\
\hline $\begin{array}{l}\text { Dissolved Organic } \\
\text { Carbon }\end{array}$ & $\mathrm{mg} / \mathrm{L}$ & 4.1 & - & Archived \\
\hline Diuron & $\mathrm{mg} / \mathrm{L}$ & 0.01 & 0.001 & 0.15 \\
\hline Escherichia coli & $\mathrm{CFU} / 100 \mathrm{~mL}$ & 0 & 0 & 0 \\
\hline Ethylbenzene & $\mathrm{mg} / \mathrm{L}$ & - & - & 0.0024 \\
\hline Fluoride & $\mathrm{mg} / \mathrm{L}$ & 0.1 & 0.03 & 1.5 \\
\hline Free Chlorine & $\mathrm{mg} / \mathrm{L}$ & $0.94^{49}$ & - & - \\
\hline Glyphosate & $\mathrm{mg} / \mathrm{L}$ & 0.01 & 0.005 & 0.28 \\
\hline Hardness & $\mathrm{mg} / \mathrm{L}$ & $110^{50}$ & - & $80-100$ \\
\hline $\begin{array}{l}\text { Heptachlor \& } \\
\text { Hephtachlor Epoxide }\end{array}$ & $\mathrm{mg} / \mathrm{L}$ & 0.00001 & 0.0002 & Archived \\
\hline Iron & $\mathrm{mg} / \mathrm{L}$ & 0.1 & - & 0.3 \\
\hline Lead & $\mathrm{mg} / \mathrm{L}$ & 0.0005 & N/A & 0.01 \\
\hline Lindane & $\mathrm{mg} / \mathrm{L}$ & 0.000006 & - & Archived \\
\hline Magnesium & $\mathrm{mg} / \mathrm{L}$ & 6.5 & - & - \\
\hline Malathion & $\mathrm{mg} / \mathrm{L}$ & 0.005 & 0.0001 & 0.19 \\
\hline Manganese & $\mathrm{mg} / \mathrm{L}$ & 0.016 & - & 0.05 \\
\hline Mercury & $\mathrm{mg} / \mathrm{L}$ & 0.0001 & 0.0001 & 0.001 \\
\hline Methyl t-butyl ether & $\mathrm{mg} / \mathrm{L}$ & - & - & 0.015 \\
\hline Methoxychlor & $\mathrm{mg} / \mathrm{L}$ & 0.00002 & 0.0001 & 0.9 \\
\hline Metolachlor & $\mathrm{mg} / \mathrm{L}$ & 0.0005 & 0.0001 & 0.05 \\
\hline Metribuzin & $\mathrm{mg} / \mathrm{L}$ & 0.005 & 0.0001 & 0.08 \\
\hline Monochlorobenzene & $\mathrm{mg} / \mathrm{L}$ & 0.0001 & 0.0005 & 0.08 \\
\hline Nickel & $\mathrm{mg} / \mathrm{L}$ & 0.004 & - & - \\
\hline Nitrate & $\mathrm{mg} / \mathrm{L}$ & 0.1 & 0.103 & 4.5 \\
\hline Nitrate + Nitrite & $\mathrm{mg} / \mathrm{L}$ & 0.1 & 0.105 & 10 \\
\hline
\end{tabular}

\footnotetext{
${ }^{49}$ Does not meet minimum requirements of the GCDWQ; however, meets requirements of the Safe Drinking Water Act, 2002.

${ }^{50}$ Exceeds maximum allowable concentration parameters within the GCDWQ.
} 


\begin{tabular}{lcccc}
\hline Nitrite & $\mathrm{mg} / \mathrm{L}$ & 0.01 & 0.02 & 1 \\
Paraquat & $\mathrm{mg} / \mathrm{L}$ & 0.001 & 0.001 & 0.01 \\
Parathion & $\mathrm{mg} / \mathrm{L}$ & 0.001 & 0.0001 & 0.05 \\
Pentachlorophenol & $\mathrm{mg} / \mathrm{L}$ & 0.0005 & 0.0005 & 0.06 \\
$\mathrm{pH}$ & $\mathrm{pH}$ & 7.4 & - & $6.5-8.5$ \\
Phorate & $\mathrm{mg} / \mathrm{L}$ & 0.0005 & 0.0001 & 0.002 \\
Picloram & $\mathrm{mg} / \mathrm{L}$ & 0.005 & 0.0002 & 0.19 \\
Polychlorinated & $\mathrm{mg} / \mathrm{L}$ & 0.00005 & 0.00002 & Archived \\
Biphenyls & $\mathrm{mg} / \mathrm{L}$ & 0.0003 & 0.0001 & - \\
Prometryne & $\mathrm{mg} / \mathrm{L}$ & 0.002 & 0.005 & 0.01 \\
Selenium & $\mathrm{mg} / \mathrm{L}$ & 0.001 & 0.0001 & 0.01 \\
Simazine & $\mathrm{mg} / \mathrm{L}$ & 1.8 & 2.62 & 20.0 \\
Sodium & $\mathrm{mg} / \mathrm{L}$ & 68 & - & 500 \\
Sulphate & $\mathrm{mg} / \mathrm{L}$ & 0.01 & - & Archived \\
Temephos & $\mathrm{mg} / \mathrm{L}$ & 0.0005 & - & 0.001 \\
Terbufos & $\mathrm{mg} / \mathrm{L}$ & 0.0001 & 0.0005 & 0.03 \\
Tetrachloroethylene & $\mathrm{mg} / \mathrm{L}$ & 0.0005 & - & 0.03 \\
Tetrachlorophenol & $\mathrm{mg} / \mathrm{L}$ & 0.0002 & - & 0.024 \\
Toluene & $\mathrm{mg} / \mathrm{L}$ & 150 & - & 500 \\
Total Dissolved Solids & $\mathrm{mg} / \mathrm{L}$ & 0.001 & 0.0001 & Archived \\
Triallate & $\mathrm{mg} / \mathrm{L}$ & 0.0005 & 0.0005 & 0.005 \\
Trichloroethylene & $\mathrm{mg} / \mathrm{L}$ & 0.0005 & 0.0005 & 0.005 \\
Trichlorophenol & $\mathrm{mg} / \mathrm{L}$ & 0.001 & 0.0002 & Archived \\
Trichlorophenoxy & $\mathrm{mg} / \mathrm{L}$ & 0.001 & 0.0001 & 0.045 \\
Acetic Acid & & 0.0361 & .0406 & 0.1 \\
Trifluralin & $\mathrm{NTU}$ & $0.90-1.01$ & - & 1.0 \\
Trihalomethanes & $\mathrm{mg} / \mathrm{L}$ & 0.0001 & 0.005 & 0.02 \\
Turbidity & $\mathrm{mg} / \mathrm{L}$ & 0.0002 & 0.0005 & 0.002 \\
Uranium & $\mathrm{mg} / \mathrm{L}$ & - & - & 0.3 \\
Vinyl Chloride & $\mathrm{mg} / \mathrm{L}$ & 0.006 & - & 5 \\
Xylenes & & & & \\
Zinc & & & & \\
\hline & & & & \\
\hline
\end{tabular}




\section{Annex 12: Drinking Water System Improvements for Longlac}

\begin{tabular}{lr}
\hline Type of Improvement Expense & \multicolumn{1}{c}{ Cost } \\
\hline Incurred Expenses (in chronological order) & \\
Filter Media Replacement & 3,226 \\
Clean Filters and Under-Drains & 2,340 \\
New Portable Hatch Colorimiter & 1,636 \\
Paint Equipment & 27,403 \\
Clean Water Tower & 7,975 \\
Clean Surge Pit & 2,143 \\
Valve Replacement & 2,0327 \\
New Lab Equipment & 6,641 \\
New Emergency Eyewash and Shower Station & 3,365 \\
New Labeller & 2,327 \\
Total & $\$ 59,093$ \\
\hline
\end{tabular}


Annex 13: Drinking Water System Improvements for Ginoogaming

\begin{tabular}{lr}
\hline Type of Improvement Expense & Cost \\
\hline Immediate Recommendations to Comply with the Protocol & $\$$ \\
Create Emergency Response Plan & 10,000 \\
& 35,000 \\
Leakage Study & 3,000 \\
Test and Calibrate Monitoring Equipment & 9,500 \\
New Distribution and Sampling Equipment & 10,000 \\
Train the Ginoogaming Primary Water Treatment Operator & 20,000 \\
Create a Maintenance Management System & $\$$ \\
Total & 84,500 \\
\hline
\end{tabular}


Annex 14: Greater Sudbury Organizational Chart: Water/Wastewater Services

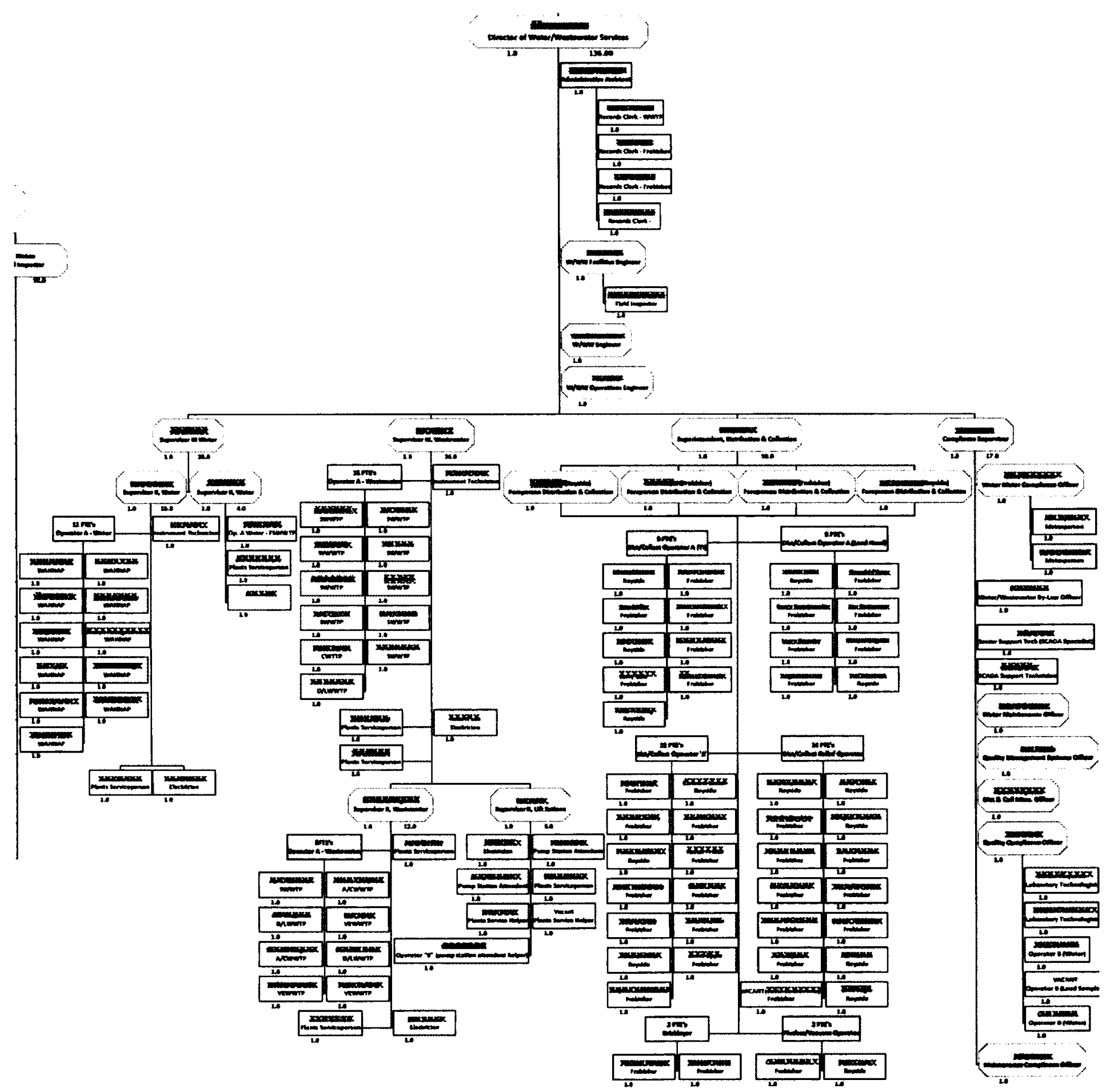


Annex 15: Water Quality Parameters for Groundwater Source

\begin{tabular}{|c|c|c|c|c|c|}
\hline \multirow[t]{2}{*}{ Parameter } & \multirow{2}{*}{$\begin{array}{l}\text { Unit of } \\
\text { Measure }\end{array}$} & \multirow{2}{*}{$\begin{array}{l}\text { Sagamok } \\
\text { Anishnawbek } \\
\text { First } \\
\text { Nation } \\
1,52 \\
\end{array}$} & \multicolumn{2}{|c|}{ Dowling ${ }^{33}$} & \multirow{2}{*}{$\begin{array}{l}\text { Maximum } \\
\text { Allowable } \\
\text { Concentrations }{ }^{54}\end{array}$} \\
\hline & & & Lionel & Riverside & \\
\hline Alachlor & $\mathrm{mg} / \mathrm{L}$ & - & 0.0005 & 0.00052 & - \\
\hline Adicarb & $\mathrm{mg} / \mathrm{L}$ & - & 0.00065 & 0.00056 & 0.009 \\
\hline Aldrin + Deildrin & $\mathrm{mg} / \mathrm{L}$ & 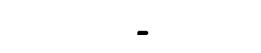 & 0.000004 & 0.000004 & 0.0007 \\
\hline Alkalinity & $\mathrm{mg} / \mathrm{L}$ & 57 & - & - & $30-500$ \\
\hline Aluminum & $\mathrm{mg} / \mathrm{L}$ & 0.011 & - & - & $0.1 / 0.2$ \\
\hline Antimony & $\mathrm{mg} / \mathrm{L}$ & 0.0005 & 0.002 & 0.0005 & 0.006 \\
\hline Arsenic & $\mathrm{mg} / \mathrm{L}$ & 0.001 & 0.001 & 0.001 & 0.01 \\
\hline $\begin{array}{l}\text { Atrazine }+\mathrm{N}- \\
\text { dealkylated metabolites }\end{array}$ & $\mathrm{mg} / \mathrm{L}$ & - & 0.0009 & 0.0009 & 0.005 \\
\hline Azinphos-methyl & $\mathrm{mg} / \mathrm{L}$ & - & 0.00038 & 0.00039 & 0.02 \\
\hline Background Bact & $\begin{array}{c}\mathrm{CFU} / 100 \mathrm{~m} \\
\mathrm{~L}\end{array}$ & 0 & - & - & - \\
\hline Barium & $\mathrm{mg} / \mathrm{L}$ & 0.006 & 0.0152 & 0.01 & 1 \\
\hline Bendiocarb & $\mathrm{mg} / \mathrm{L}$ & - & 0.0013 & 0.0011 & 0.04 \\
\hline Benzene & $\mathrm{mg} / \mathrm{L}$ & 0.0001 & 0.00025 & 0.0025 & 0.005 \\
\hline Benzo(a)pyrene & $\mathrm{mg} / \mathrm{L}$ & - & 0.000006 & 0.000006 & 0.00001 \\
\hline Bromodichloromethane & $\mathrm{mg} / \mathrm{L}$ & 0.0002 & 0.000006 & 0.000006 & 0.01 \\
\hline Bromoform & $\mathrm{mg} / \mathrm{L}$ & 0.0002 & 0.00067 & 0.0005 & 0.005 \\
\hline Bromoxynil & $\mathrm{mg} / \mathrm{L}$ & - & 0.00067 & 0.0005 & 0.005 \\
\hline Boron & $\mathrm{mg} / \mathrm{L}$ & 0.01 & 0.018 & 0.0063 & 5 \\
\hline Cadmium & $\mathrm{mg} / \mathrm{L}$ & 0.0001 & 0.0001 & 0.0001 & 0.005 \\
\hline Carbaryl & $\mathrm{mg} / \mathrm{L}$ & - & 0.0013 & 0.0011 & 0.09 \\
\hline Carbofuran & $\mathrm{mg} / \mathrm{L}$ & - & 0.0013 & 0.0011 & 0.09 \\
\hline Carbon Tetrachloride & $\mathrm{mg} / \mathrm{L}$ & 0.0001 & 0.00025 & 0.00025 & 0.005 \\
\hline Chloride & $\mathrm{mg} / \mathrm{L}$ & 12 & $\begin{array}{c}0.49- \\
2.45\end{array}$ & $\begin{array}{c}0.34- \\
3.09\end{array}$ & 250 \\
\hline Chlorobenzene & $\mathrm{mg} / \mathrm{L}$ & 0.0001 & - & - & - \\
\hline Chlorodane & $\mathrm{mg} / \mathrm{L}$ & - & 0.000004 & 0.000004 & Archived $^{55}$ \\
\hline Chlorpyrifos & $\mathrm{mg} / \mathrm{L}$ & - & 0.00038 & 0.00039 & 0.09 \\
\hline Chromium & $\mathrm{mg} / \mathrm{L}$ & 0.005 & 0.001 & 0.001 & 0.05 \\
\hline Coliform & $\underset{\mathrm{L}}{\mathrm{CFU} / 100 \mathrm{~m}}$ & 0 & 0 & $0^{56}$ & 0 \\
\hline Conductivity & umhos $/ \mathrm{cm}$ & 186 & - & - & - \\
\hline
\end{tabular}

${ }^{51}$ Obtained from Neegan Burnside via the engineer report (Paznar, 2011).

52 Only parameters for the Halfway pumphouse are shown, as the Toulouse was not in operation during the Neegan Burnside assessment.

${ }^{53}$ Obtained from Greater Sudbury's (2010b) website. These results are required to be public as per O.Reg. 170/03 under the Safe Drinking Water Act, 2002.

${ }_{54}^{5}$ Obtained from the Guidelines of Canadian Drinking Water Quality: Summary Table (HC, 2010c).

${ }^{55}$ Archived measures are no longer part of the GCDWQ.

${ }^{56} \mathrm{~A}$ raw water sample found $1 \mathrm{CFU} / 100 \mathrm{~mL}$ 


\begin{tabular}{|c|c|c|c|c|c|}
\hline Copper & $\mathrm{mg} / \mathrm{L}$ & 0.032 & - & - & 1.0 \\
\hline Cyanazine & $\mathrm{mg} / \mathrm{L}$ & - & 0.00038 & 0.00039 & 0.01 \\
\hline Diazinon & $\mathrm{mg} / \mathrm{L}$ & - & 0.00038 & 0.00039 & 0.02 \\
\hline Dicambia & $\mathrm{mg} / \mathrm{L}$ & - & 0.00027 & 0.0002 & 0.12 \\
\hline Dibromochoromethane & $\mathrm{mg} / \mathrm{L}$ & 0.0002 & - & - & - \\
\hline Dichorobenzene $(1,2)$ & $\mathrm{mg} / \mathrm{L}$ & 0.0002 & 0.00025 & 0.00025 & 0.2 \\
\hline Dichorobenzene $(1,4)$ & $\mathrm{mg} / \mathrm{L}$ & 0.0002 & 0.00025 & 0.00025 & 0.005 \\
\hline $\begin{array}{l}\text { Dichlorodiphenyltri- } \\
\text { chloromethate (DDT) } \\
\text { + metobolites }\end{array}$ & $\mathrm{mg} / \mathrm{L}$ & - & 0.000005 & 0.000005 & Archived \\
\hline Dichloroethane $(1,2)$ & $\mathrm{mg} / \mathrm{L}$ & 0.0002 & 0.00025 & 0.00025 & 0.005 \\
\hline Dichloroethylene $(1,1)$ & $\mathrm{mg} / \mathrm{L}$ & 0.0001 & 0.00025 & 0.00025 & 0.014 \\
\hline Dichloromethane & $\mathrm{mg} / \mathrm{L}$ & 0.002 & 0.00025 & 0.00025 & 0.05 \\
\hline Dichlorophenol & $\mathrm{mg} / \mathrm{L}$ & - & 0.000059 & 0.000058 & 0.9 \\
\hline $\begin{array}{l}\text { Dichlorophenoxy } \\
\text { acetic acid }\end{array}$ & $\mathrm{mg} / \mathrm{L}$ & - & 0.00027 & 0.0002 & 0.1 \\
\hline Diclofop-methyl & $\mathrm{mg} / \mathrm{L}$ & - & 0.00027 & 0.0002 & 0.009 \\
\hline Dimethoate & $\mathrm{mg} / \mathrm{L}$ & - & 0.00038 & 0.00039 & 0.02 \\
\hline Dinoseb & $\mathrm{mg} / \mathrm{L}$ & - & 0.000067 & 0.00005 & 0.01 \\
\hline Diquat & $\mathrm{mg} / \mathrm{L}$ & - & 0.007 & 0.007 & 0.07 \\
\hline Diuron & $\mathrm{mg} / \mathrm{L}$ & - & 0.0065 & 0.0056 & 0.15 \\
\hline Escherichia coli & $\begin{array}{c}\mathrm{CFU} / 100 \mathrm{~m} \\
\mathrm{~L}\end{array}$ & 0 & 0 & 0 & 0 \\
\hline Ethylbenzene & $\mathrm{mg} / \mathrm{L}$ & 0.0001 & - & - & 0.0024 \\
\hline Fluoride & $\mathrm{mg} / \mathrm{L}$ & 0.1 & $\begin{array}{c}0.20- \\
1.18\end{array}$ & $\begin{array}{c}0.20 \\
1.26\end{array}$ & 1.5 \\
\hline Glyphosate & $\mathrm{mg} / \mathrm{L}$ & - & 0.02 & 0.02 & 0.28 \\
\hline Hardness & $\mathrm{mg} / \mathrm{L}$ & 72 & - & - & $80-100$ \\
\hline $\begin{array}{l}\text { Heptachlor }+ \\
\text { Heptachlor Epoxide }\end{array}$ & $\mathrm{mg} / \mathrm{L}$ & - & 0.000004 & 0.000004 & Archived \\
\hline Iron & $\mathrm{mg} / \mathrm{L}$ & 0.3 & - & - & 0.3 \\
\hline Lead & $\mathrm{mg} / \mathrm{L}$ & 0.001 & 0.001 & 0.001 & 0.01 \\
\hline Linadane & $\mathrm{mg} / \mathrm{L}$ & - & 0.000005 & 0.000006 & Archived \\
\hline Malathion & $\mathrm{mg} / \mathrm{L}$ & - & 0.00038 & 0.00039 & 0.19 \\
\hline Manganese & $\mathrm{mg} / \mathrm{L}$ & 0.002 & - & - & 0.05 \\
\hline Mercury & $\mathrm{mg} / \mathrm{L}$ & 0.0001 & 0.00001 & 0.00001 & 0.001 \\
\hline Methoxychlor & $\mathrm{mg} / \mathrm{L}$ & - & 0.000002 & 0.000002 & 0.9 \\
\hline Methyl t-butyl ether & $\mathrm{mg} / \mathrm{L}$ & 0.0002 & - & - & 0.015 \\
\hline Metolachlor & $\mathrm{mg} / \mathrm{L}$ & - & 0.00025 & 0.00026 & 0.05 \\
\hline Metribuzin & $\mathrm{mg} / \mathrm{L}$ & - & 0.00025 & 0.00026 & 0.08 \\
\hline Monochlorobenzene & $\mathrm{mg} / \mathrm{L}$ & - & 0.00025 & 0.00025 & 0.08 \\
\hline Nitrate & $\mathrm{mg} / \mathrm{L}$ & 0.5 & 0.41 & 0.23 & 4.5 \\
\hline Nitrate + Nitrite & $\mathrm{mg} / \mathrm{L}$ & 0.5 & - & - & 10 \\
\hline Nitrite & $\mathrm{mg} / \mathrm{L}$ & 0.01 & 0.05 & .05 & 1 \\
\hline o-Xylene & $\mathrm{mg} / \mathrm{L}$ & 0.0001 & - & - & 0.3 \\
\hline $\mathrm{p}+\mathrm{m}$-Xylene & $\mathrm{mg} / \mathrm{L}$ & 0.0001 & - & - & 0.3 \\
\hline
\end{tabular}




\begin{tabular}{|c|c|c|c|c|c|}
\hline Paraquat & $\mathrm{mg} / \mathrm{L}$ & - & 0.001 & 0.001 & 0.01 \\
\hline Parathion & $\mathrm{mg} / \mathrm{L}$ & - & 0.00025 & 0.00026 & 0.05 \\
\hline Pentachlorophenol & $\mathrm{mg} / \mathrm{L}$ & - & 0.000059 & 0.000058 & 0.06 \\
\hline $\mathrm{pH}$ & $\mathrm{pH}$ & 7.4 & - & - & $6.5-8.5$ \\
\hline Phorate & $\mathrm{mg} / \mathrm{L}$ & - & 0.00038 & 0.00039 & 0.002 \\
\hline Phosphorus & $\mathrm{mg} / \mathrm{L}$ & 0.1 & - & - & - \\
\hline Picloram & $\mathrm{mg} / \mathrm{L}$ & - & 0.000067 & 0.00005 & 0.19 \\
\hline $\begin{array}{l}\text { Polychlorinated } \\
\text { Biphenyls }\end{array}$ & $\mathrm{mg} / \mathrm{L}$ & - & 0.000005 & 0.000052 & Archived \\
\hline Prometryne & $\mathrm{mg} / \mathrm{L}$ & - & 0.00025 & 0.00026 & - \\
\hline Selenium & $\mathrm{mg} / \mathrm{L}$ & 0.002 & 0.001 & 0.001 & 0.01 \\
\hline Simazine & $\mathrm{mg} / \mathrm{L}$ & - & 0.00038 & 0.00039 & 0.01 \\
\hline Sodium & $\mathrm{mg} / \mathrm{L}$ & 6.6 & 22.2 & 35.9 & 20.0 \\
\hline Sulphate & $\mathrm{mg} / \mathrm{L}$ & 12 & - & - & 500 \\
\hline Temephos & $\mathrm{mg} / \mathrm{L}$ & - & 0.018 & 0.016 & Archived \\
\hline Terbufos & $\mathrm{mg} / \mathrm{L}$ & - & 0.00025 & 0.00026 & 0.001 \\
\hline Tetrachloroethylene & $\mathrm{mg} / \mathrm{L}$ & 0.0001 & - & - & 0.03 \\
\hline Tetrachlorophenol & $\mathrm{mg} / \mathrm{L}$ & - & 0.000059 & 0.000058 & 0.03 \\
\hline Toluene & $\mathrm{mg} / \mathrm{L}$ & 0.0002 & - & - & 0.024 \\
\hline Total Dissolved Solids & $\mathrm{mg} / \mathrm{L}$ & 120 & - & - & 500 \\
\hline Triallate & $\mathrm{mg} / \mathrm{L}$ & - & 0.00025 & 0.00026 & Archived \\
\hline Trichloroethylene & $\mathrm{mg} / \mathrm{L}$ & 0.0001 & 0.00025 & 0.00025 & 0.005 \\
\hline Trichlorophenol & $\mathrm{mg} / \mathrm{L}$ & - & 0.000059 & 0.000058 & 0.005 \\
\hline $\begin{array}{l}\text { Trichlorophenoxy } \\
\text { acetic acid }\end{array}$ & $\mathrm{mg} / \mathrm{L}$ & - & 0.000067 & 0.00005 & Archived \\
\hline Trifluralin & $\mathrm{mg} / \mathrm{L}$ & - & 0.00025 & 0.00026 & 0.045 \\
\hline Uranium & $\mathrm{mg} / \mathrm{L}$ & 0.0015 & 0.001 & 0.001 & 0.02 \\
\hline Vinyl Chloride & $\mathrm{mg} / \mathrm{L}$ & 0.0002 & 0.00025 & 0.00025 & 0.002 \\
\hline Xylenes & $\mathrm{mg} / \mathrm{L}$ & 0.0001 & - & - & 0.3 \\
\hline Zinc & $\mathrm{mg} / \mathrm{L}$ & 0.005 & - & - & 5 \\
\hline
\end{tabular}


Annex 16: Drinking Water System Improvements for Dowling

\begin{tabular}{lr}
\hline Incurred Improvement Expenses (in chronological order) & Cost \\
\hline Type of Improvement & \\
New Ultra-Violet Irradiation System & N/A \\
New Chlorine Switch-Over Equipment & 55,000 \\
Tank Repairs & 60,000 \\
Total & $\$ 115,000$ \\
\hline
\end{tabular}




\section{Annex 17: Drinking Water System Improvements for Sagamok}

\begin{tabular}{lr}
\hline Immediate Recommendations to Comply with the Protocol & Cost \\
\hline Type of Improvement & $\$$ \\
Additional Piping Infrastructure & 130,000 \\
& 40,000 \\
Expanding the Pumphouse Buildings & 25,000 \\
Fence around each Pumphouse & 30,000 \\
Create Source Water Protection Plan & 15,000 \\
Create Operation and Maintenance Manual & 10,000 \\
Update the Emergency Response Plan & 55,000 \\
Conduct Hydrogeological Study & 13,000 \\
New Standby Power Unit & 20,000 \\
Train the Water Treatment Plant Operators & 25,000 \\
Calibrate Monitoring Equipment & 2,500 \\
Create Quality Assurance Monitoring and Sampling Program & 8,000 \\
Create Source Water Monitoring Program & 8,000 \\
Create Distribution Monitoring Program & 50,000 \\
Engineering and Contingency Costs & $\$$ \\
Total & 431,500 \\
\hline
\end{tabular}


Annex 18: Water Quality Parameters for Surface Water Source

\begin{tabular}{|c|c|c|c|c|}
\hline Parameter & $\begin{array}{l}\text { Unit of } \\
\text { Measure }\end{array}$ & $\begin{array}{l}\text { Pikangikum } \\
\text { First } \\
\text { Nation }\end{array}$ & Red Lake $^{58}$ & $\begin{array}{c}\text { Maximum } \\
\text { Allowable } \\
\text { Concentrations }\end{array}$ \\
\hline Alachlor & $\mathrm{mg} / \mathrm{L}$ & - & 0.0001 & - \\
\hline Aldicarb & $\mathrm{mg} / \mathrm{L}$ & - & 0.001 & 0.009 \\
\hline Aldin + Dieldrin & $\mathrm{mg} / \mathrm{L}$ & - & 0.00004 & 0.0007 \\
\hline Alkalinity & $\mathrm{mg} / \mathrm{L}$ & - & 38.0 & $30-500$ \\
\hline Aluminum & $\mathrm{mg} / \mathrm{L}$ & - & 0.057 & $0.1 / 0.2$ \\
\hline Antimony & $\mathrm{mg} / \mathrm{L}$ & - & 0.0006 & 0.006 \\
\hline Arsenic & $\mathrm{mg} / \mathrm{L}$ & - & 0.001 & 0.01 \\
\hline $\begin{array}{l}\text { Atrazine }+\mathrm{N}- \\
\text { dealkylated metabolites }\end{array}$ & $\mathrm{mg} / \mathrm{L}$ & - & 0.0002 & 0.005 \\
\hline Azinphos-methyl & $\mathrm{mg} / \mathrm{L}$ & - & 0.0001 & 0.02 \\
\hline Barium & $\mathrm{mg} / \mathrm{L}$ & - & 0.01 & 1 \\
\hline Bendiocarb & $\mathrm{mg} / \mathrm{L}$ & - & 0.0002 & 0.04 \\
\hline Benzene & $\mathrm{mg} / \mathrm{L}$ & - & 0.0005 & 0.005 \\
\hline Benzo(a)pyrene & $\mathrm{mg} / \mathrm{L}$ & - & 0.00001 & 0.00001 \\
\hline Boron & $\mathrm{mg} / \mathrm{L}$ & - & 0.05 & 5 \\
\hline Bromodichloromethane & $\mathrm{mg} / \mathrm{L}$ & - & 0.00001 & 0.01 \\
\hline Bromoform & $\mathrm{mg} / \mathrm{L}$ & - & 0.0002 & 0.005 \\
\hline Bromoxynil & $\mathrm{mg} / \mathrm{L}$ & - & 0.0002 & 0.005 \\
\hline Cadmium & $\mathrm{mg} / \mathrm{L}$ & - & 0.0001 & 0.005 \\
\hline Carbaryl & $\mathrm{mg} / \mathrm{L}$ & - & 0.0002 & 0.09 \\
\hline Carbofuran & $\mathrm{mg} / \mathrm{L}$ & - & 0.0002 & 0.09 \\
\hline Carbon Tetrachloride & $\mathrm{mg} / \mathrm{L}$ & - & 0.0005 & 0.005 \\
\hline Chlordane & $\mathrm{mg} / \mathrm{L}$ & - & 0.0003 & Archived $^{60}$ \\
\hline Chlorobenzene & $\mathrm{mg} / \mathrm{L}$ & - & 0.0003 & - \\
\hline Chlorpyrifos & $\mathrm{mg} / \mathrm{L}$ & - & 0.0001 & 0.09 \\
\hline Chromium & $\mathrm{mg} / \mathrm{L}$ & - & 0.001 & 0.05 \\
\hline Coliform & $\mathrm{CFU} / 100 \mathrm{~mL}$ & - & 0 & 0 \\
\hline Cyanazine & $\mathrm{mg} / \mathrm{L}$ & - & 0.0001 & 0.01 \\
\hline Diazinon & $\mathrm{mg} / \mathrm{L}$ & - & 0.0001 & 0.02 \\
\hline Dicamba & $\mathrm{mg} / \mathrm{L}$ & - & 0.0002 & 0.12 \\
\hline Dichorobenzene $(1,2)$ & $\mathrm{mg} / \mathrm{L}$ & - & 0.0005 & 0.2 \\
\hline Dichorobenzene $(1,4)$ & $\mathrm{mg} / \mathrm{L}$ & - & 0.0005 & 0.005 \\
\hline DDT + metabolites & $\mathrm{mg} / \mathrm{L}$ & - & 0.0004 & Archived \\
\hline Dichloroethane $(1,2)$ & $\mathrm{mg} / \mathrm{L}$ & - & 0.0005 & 0.005 \\
\hline Dichloroethylene $(1,1)$ & $\mathrm{mg} / \mathrm{L}$ & - & 0.0005 & 0.014 \\
\hline
\end{tabular}

\footnotetext{
${ }^{57}$ Data from Pikangikum and $\mathrm{HC}$ was not made available for the National Assessment of First Nations Water and Wastewater Systems (Mayberry, 2011). Neegan Burnside did not conduct its own analysis. ${ }^{58}$ Obtained from the Municipality of Red Lake's website (Northern Waterworks, 2011). These results are required to be made publically available as per O.Reg. 170/03 under the Safe Drinking Water Act, 2002.

${ }^{59}$ Obtained from the Guidelines of Canadian Drinking Water Quality: Summary Table (HC, 2010).

${ }^{60}$ Archived measures are no longer part of the GCDWQ.
} 


\begin{tabular}{|c|c|c|c|c|}
\hline Dichloromethane & $\mathrm{mg} / \mathrm{L}$ & - & 0.0005 & 0.05 \\
\hline Dichlorophenol & $\mathrm{mg} / \mathrm{L}$ & - & 0.0003 & 0.9 \\
\hline $\begin{array}{l}\text { Dichlorophenolxy } \\
\text { acetic acid }\end{array}$ & $\mathrm{mg} / \mathrm{L}$ & - & 0.0002 & 0.1 \\
\hline Diclofop-methyl & $\mathrm{mg} / \mathrm{L}$ & - & 0.0002 & 0.009 \\
\hline Dimethoate & $\mathrm{mg} / \mathrm{L}$ & - & 0.0001 & 0.02 \\
\hline Dinoseb & $\mathrm{mg} / \mathrm{L}$ & - & 0.0002 & 0.01 \\
\hline Diquat & $\mathrm{mg} / \mathrm{L}$ & - & 0.001 & 0.07 \\
\hline Diuron & $\mathrm{mg} / \mathrm{L}$ & - & 0.001 & 0.15 \\
\hline Escherichia coli & $\mathrm{CFU} / 100 \mathrm{~mL}$ & - & 0 & 0 \\
\hline Fluoride & $\mathrm{mg} / \mathrm{L}$ & - & 0.03 & 1.5 \\
\hline Glyphosate & $\mathrm{mg} / \mathrm{L}$ & - & 0.05 & 0.28 \\
\hline Heptachlor + Epoxide & $\mathrm{mg} / \mathrm{L}$ & - & 0.0002 & Archived \\
\hline Lead & $\mathrm{mg} / \mathrm{L}$ & - & 0.001 & 0.01 \\
\hline Lindane & $\mathrm{mg} / \mathrm{L}$ & - & 0.0001 & Archived \\
\hline Malathion & $\mathrm{mg} / \mathrm{L}$ & - & 0.0001 & 0.19 \\
\hline Mercury & $\mathrm{mg} / \mathrm{L}$ & - & 0.0001 & 0.001 \\
\hline Metclachlor & $\mathrm{mg} / \mathrm{L}$ & - & 0.0001 & 0.05 \\
\hline Methoxychlor & $\mathrm{mg} / \mathrm{L}$ & - & 0.0001 & 0.9 \\
\hline Metribuzin & $\mathrm{mg} / \mathrm{L}$ & - & 0.0001 & 0.08 \\
\hline Nitrate & $\mathrm{mg} / \mathrm{L}$ & - & 0.098 & 4.5 \\
\hline Nitrite & $\mathrm{mg} / \mathrm{L}$ & - & 0.02 & 1 \\
\hline Paraquat & $\mathrm{mg} / \mathrm{L}$ & - & 0.001 & 0.01 \\
\hline Parathion & $\mathrm{mg} / \mathrm{L}$ & - & 0.0001 & 0.05 \\
\hline Pentachlorophenol & $\mathrm{mg} / \mathrm{L}$ & - & 0.0005 & 0.06 \\
\hline $\mathrm{pH}$ & $\mathrm{pH}$ & - & 8.1 & $6.5-8.5$ \\
\hline Phorate & $\mathrm{mg} / \mathrm{L}$ & - & 0.0001 & 0.002 \\
\hline Picloram & $\mathrm{mg} / \mathrm{L}$ & - & 0.0002 & 0.19 \\
\hline $\begin{array}{l}\text { Polychlorinated } \\
\text { Biphenyls }\end{array}$ & $\mathrm{mg} / \mathrm{L}$ & - & 0.0000035 & 0.000052 \\
\hline Prometryne & $\mathrm{mg} / \mathrm{L}$ & - & 0.0001 & - \\
\hline Selenium & $\mathrm{mg} / \mathrm{L}$ & - & 0.005 & 0.01 \\
\hline Simazine & $\mathrm{mg} / \mathrm{L}$ & - & 0.0001 & 0.01 \\
\hline Sodium & $\mathrm{mg} / \mathrm{L}$ & - & 15.4 & 20.0 \\
\hline Temephos & $\mathrm{mg} / \mathrm{L}$ & - & 0.0001 & Archived \\
\hline Terbufos & $\mathrm{mg} / \mathrm{L}$ & - & 0.0002 & 0.001 \\
\hline Tetrachloroethylene & $\mathrm{mg} / \mathrm{L}$ & - & 0.0005 & 0.03 \\
\hline Tetrachlorophenol & $\mathrm{mg} / \mathrm{L}$ & - & 0.0005 & 0.03 \\
\hline Triallate & $\mathrm{mg} / \mathrm{L}$ & - & 0.0001 & Archived \\
\hline Trichloroethylene & $\mathrm{mg} / \mathrm{L}$ & - & 0.0005 & 0.005 \\
\hline $\begin{array}{l}\text { Trichlorophenoxy } \\
\text { acetic acid }\end{array}$ & $\mathrm{mg} / \mathrm{L}$ & - & 0.0002 & Archived \\
\hline Trifluralin & $\mathrm{mg} / \mathrm{L}$ & - & 0.0001 & 0.045 \\
\hline Trichloroethylene & $\mathrm{mg} / \mathrm{L}$ & - & 0.0005 & 0.005 \\
\hline Uranium & $\mathrm{mg} / \mathrm{L}$ & - & 0.005 & 0.02 \\
\hline Vinyl Chloride & $\mathrm{Mg} / \mathrm{L}$ & - & 0.0005 & 0.002 \\
\hline
\end{tabular}




\section{Annex 19: Drinking Water System Improvements for Red Lake}

\begin{tabular}{lr}
\hline Incurred Improvement Expenses (in chronological order) & Cost \\
\hline Type of Improvement & \\
Electrical Equipment Repairs & 3,590 \\
Mechanical Equipment Repairs & 3,068 \\
Miscellaneous Equipment Repairs & 7,251 \\
Electrical Equipment Replacement & 2,020 \\
Mechanical Equipment Replacement & 1,150 \\
Measurement Instrument Equipment Replacement & 4,321 \\
Chlorination System Repairs & 33,000 \\
High Lift Pump Replacement & 48,000 \\
Treatment Equipment Replacement & 230,000 \\
Chlorination System Replacement & 33,900 \\
High Lift Pump Maintenance & 2,500 \\
Additional Measurement Instrumentation Equipment & 2,500 \\
Total & $\$ 371,300$ \\
\hline
\end{tabular}


Annex 20: Drinking Water System Improvements for Pikangikum

\begin{tabular}{lr}
\hline Immediate Recommendations to Comply with the Protocol & \multicolumn{1}{c}{ Cost } \\
\hline Type of Improvement & $\$$ \\
Additional Train Filter & $1,730,000$ \\
& 300,000 \\
Expand the Treatment Reservoir & 170,000 \\
New Ultraviolet Disinfector & 45,000 \\
New Primary Disinfection Feed System & 13,000 \\
New Chemical Storage Shed & 17,000 \\
Upgrade the Chlorine Analyzer & 17,000 \\
Upgrade the Flow Meter & 70,000 \\
Additional Fire Pump & 130,000 \\
Standby Power Station & 640,000 \\
New Engineering and Contingency Measures & 50,000 \\
Clean the Treatment Reservoir & 10,000 \\
Upgrade the Emergency Response Plan & 40,000 \\
Create a Source Water Protection Plan & 7,500 \\
Update the Operations and Maintenance Manual & 20,000 \\
Create a Maintenance Management System & 5,000 \\
Draft schematics for the Water System & 15,000 \\
Train Water Treatment Operators & 3,000 \\
Calibrate Monitoring Equipment & 10,000 \\
Implementing a Distribution and Monitoring Program & $\$$ \\
Total & $3,292,500$ \\
\hline
\end{tabular}

